\title{
Parathyroid gland detection in hyperparathyroidism with 18F-fluorocholine PET/CT
}

Citation for published version (APA):

Broos, W. A. M. (2021). Parathyroid gland detection in hyperparathyroidism with 18F-fluorocholine PET/CT. [Doctoral Thesis, Maastricht University]. Ipskamp. https://doi.org/10.26481/dis.20210702wb

Document status and date:

Published: 01/01/2021

DOI:

10.26481/dis.20210702wb

Document Version:

Publisher's PDF, also known as Version of record

\section{Please check the document version of this publication:}

- A submitted manuscript is the version of the article upon submission and before peer-review. There can be important differences between the submitted version and the official published version of record.

People interested in the research are advised to contact the author for the final version of the publication, or visit the DOI to the publisher's website.

- The final author version and the galley proof are versions of the publication after peer review.

- The final published version features the final layout of the paper including the volume, issue and page numbers.

Link to publication

\footnotetext{
General rights rights.

- You may freely distribute the URL identifying the publication in the public portal. please follow below link for the End User Agreement:

www.umlib.nl/taverne-license

Take down policy

If you believe that this document breaches copyright please contact us at:

repository@maastrichtuniversity.nl

providing details and we will investigate your claim.
}

Copyright and moral rights for the publications made accessible in the public portal are retained by the authors and/or other copyright owners and it is a condition of accessing publications that users recognise and abide by the legal requirements associated with these

- Users may download and print one copy of any publication from the public portal for the purpose of private study or research.

- You may not further distribute the material or use it for any profit-making activity or commercial gain

If the publication is distributed under the terms of Article $25 \mathrm{fa}$ of the Dutch Copyright Act, indicated by the "Taverne" license above, 


\section{Parathyroid gland detection in hyperparathyroidism with 18F-fluorocholine PET/CT}

Wouter A.M. Broos

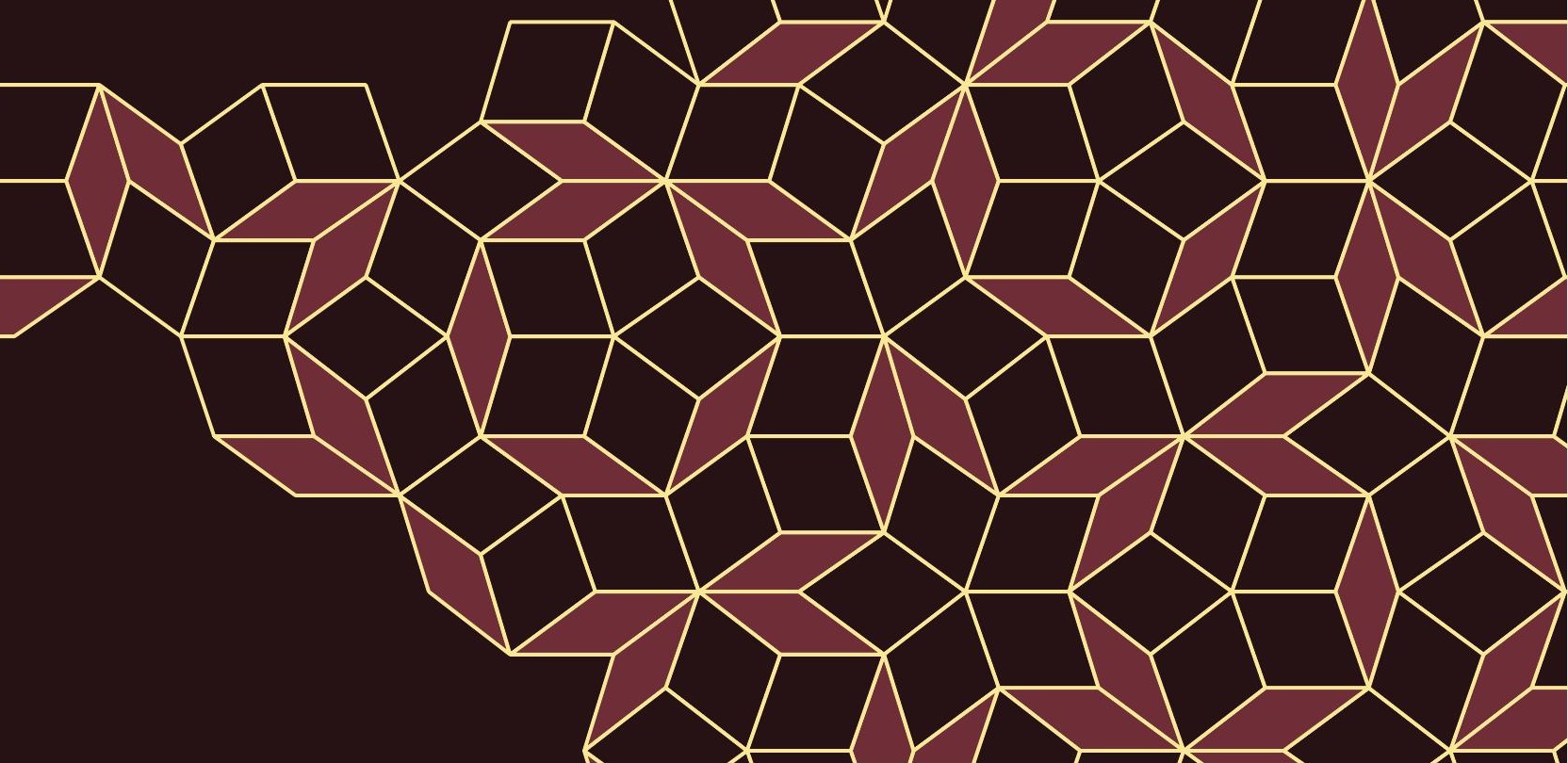





\section{Parathyroid gland detection in hyperparathyroidism with ${ }^{18}$ F-fluorocholine PET/CT}

Wouter A.M. Broos 
Parathyroid gland detection in hyperparathyroidism with ${ }^{18} \mathrm{~F}$-fluorocholine PET/CT PhD thesis, Maastricht University

(CWouter Broos, Alkmaar, 2021

w.broos@nwz.nl

All rights reserved. No part of this publication may be reproduced or transmitted in any form or by any means without permission in writing from the author. The copyright of the articles that have been published has been transferred to the respective journals.

ISBN: 978-94-6421-326-3

Cover design and lay-out: Jules Verkade, persoonlijkproefschrift.nl

Printing: Ipskamp Printing | proefschriften.net

Printing of this thesis was financially supported by:

Cyclotron Noordwest BV and Noordwest Academie, part of Noordwest Ziekenhuisgroep 


\title{
Parathyroid gland detection in hyperparathyroidism with ${ }^{18}$ F-fluorocholine PET/CT
}

\author{
PROEFSCHRIFT
}

ter verkrijging van de graad van doctor aan de Universiteit Maastricht, op gezag van de Rector Magnificus, prof. dr. Rianne M. Letschert volgens het besluit van het College van Decanen,

in het openbaar te verdedigen op vrijdag 2 juli 2021 om 12.00 uur

door

Walterus Adrianus Maria Broos Geboren op 21 september 1986 te Roosendaal en Nispen 


\section{Promotor}

Em. Prof. dr. N.C. Schaper

\section{Copromotores}

Dr. M. Wondergem (Noordwest Ziekenhuisgroep Alkmaar)

Dr. R.J.J. Knol (Noordwest Ziekenhuisgroep Alkmaar)

\section{Beoordelingscommissie}

Prof. dr. F.M. Mottaghy (Voorzitter)

Prof. dr. N.D. Bouvy

Prof. dr. A.W.J.M. Glaudemans (UMC Groningen)

Prof. dr. G.D. Valk (UMC Utrecht) 


\section{Table of contents}

Part 1 Introduction

Chapter 1 General introduction and thesis outline 9

Chapter 2 Choline PET/CT in parathyroid imaging: a systematic review 21

Part 2 Technical aspects of ${ }^{18} \mathrm{~F}$-fluorocholine PET/CT

Chapter 3 Dual-time-point ${ }^{18} \mathrm{~F}$-fluorocholine PET/CT in parathyroid imaging 47

Chapter 4 Dynamic ${ }^{18}$ F-fluorocholine PET/CT for parathyroid imaging in 63 patients with primary hyperparathyroidism

Intermezzo Tertiary hyperparathyroidism with renal osteodystrophy on $\quad 79$ ${ }^{18} \mathrm{~F}$-fluorocholine PET/CT

Part $3 \quad$ Clinical aspects of ${ }^{18} \mathrm{~F}$-fluorocholine PET/CT

Chapter 5 Parathyroid imaging with ${ }^{18} \mathrm{~F}$-fluorocholine PET/CT as a firstline imaging modality in primary hyperparathyroidism: a retrospective cohort study

Chapter 6 Incidental findings on ${ }^{18} \mathrm{~F}$-fluorocholine PET/CT for 103 parathyroid imaging

\section{Part $4 \quad$ Summary, discussion and future perspectives}

$\begin{array}{lll}\text { Chapter } 7 & \text { Thesis summary } & 121\end{array}$

$\begin{array}{lll}\text { Chapter } 8 \text { General discussion } & 125\end{array}$

$\begin{array}{lll}\text { Chapter } 9 & \text { Impact paragraph } & 155\end{array}$

\section{Appendices}

Appendix 1 Nederlandse samenvatting 162

$\begin{array}{ll}\text { Appendix } 2 \text { Abbreviations } & 168\end{array}$

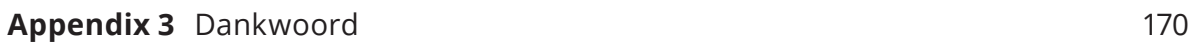

Appendix 4 List of publications 172

$\begin{array}{ll}\text { Appendix } 5 \text { Curriculum vitae } & 174\end{array}$ 


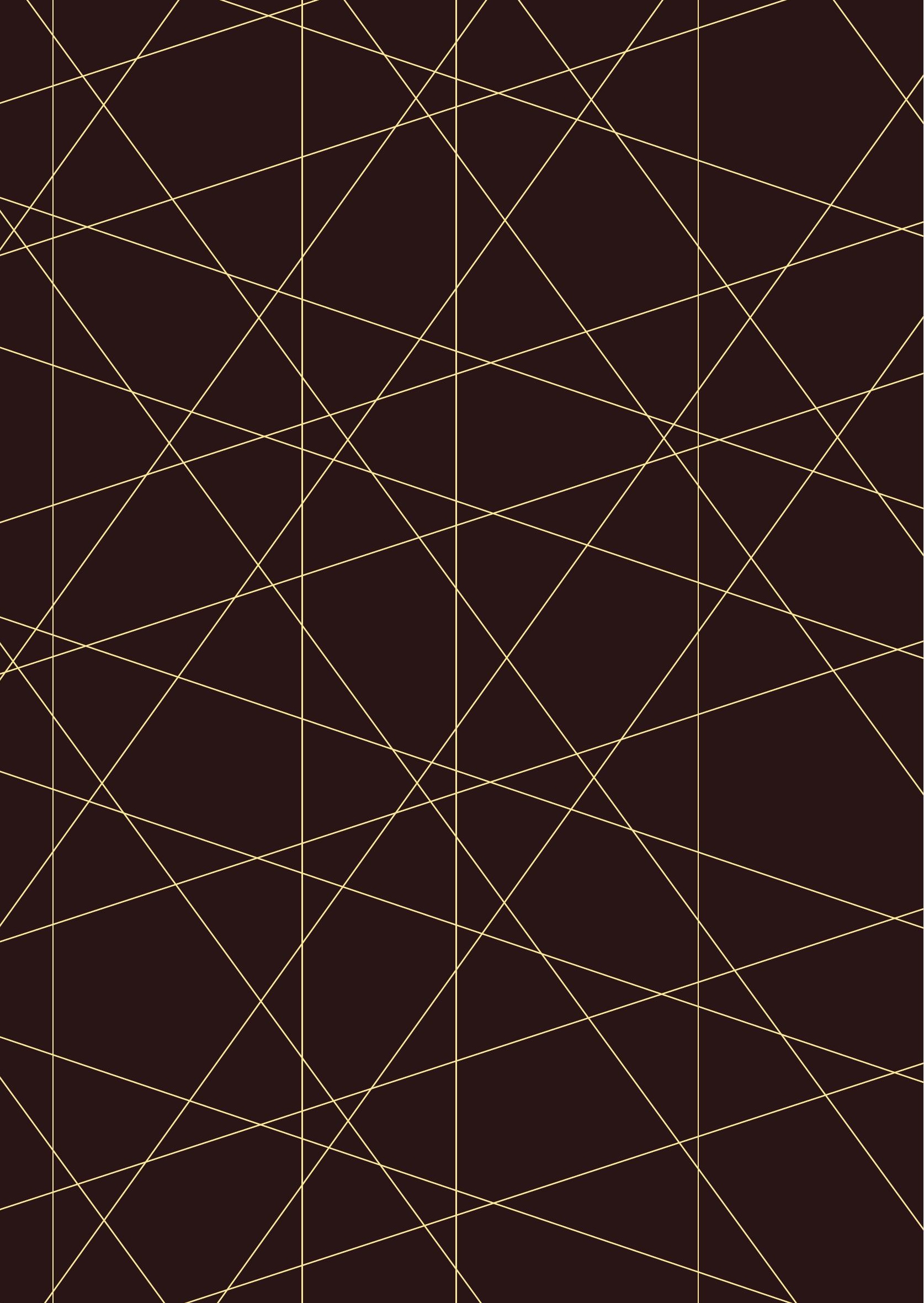




\section{PART 1}

Introduction 


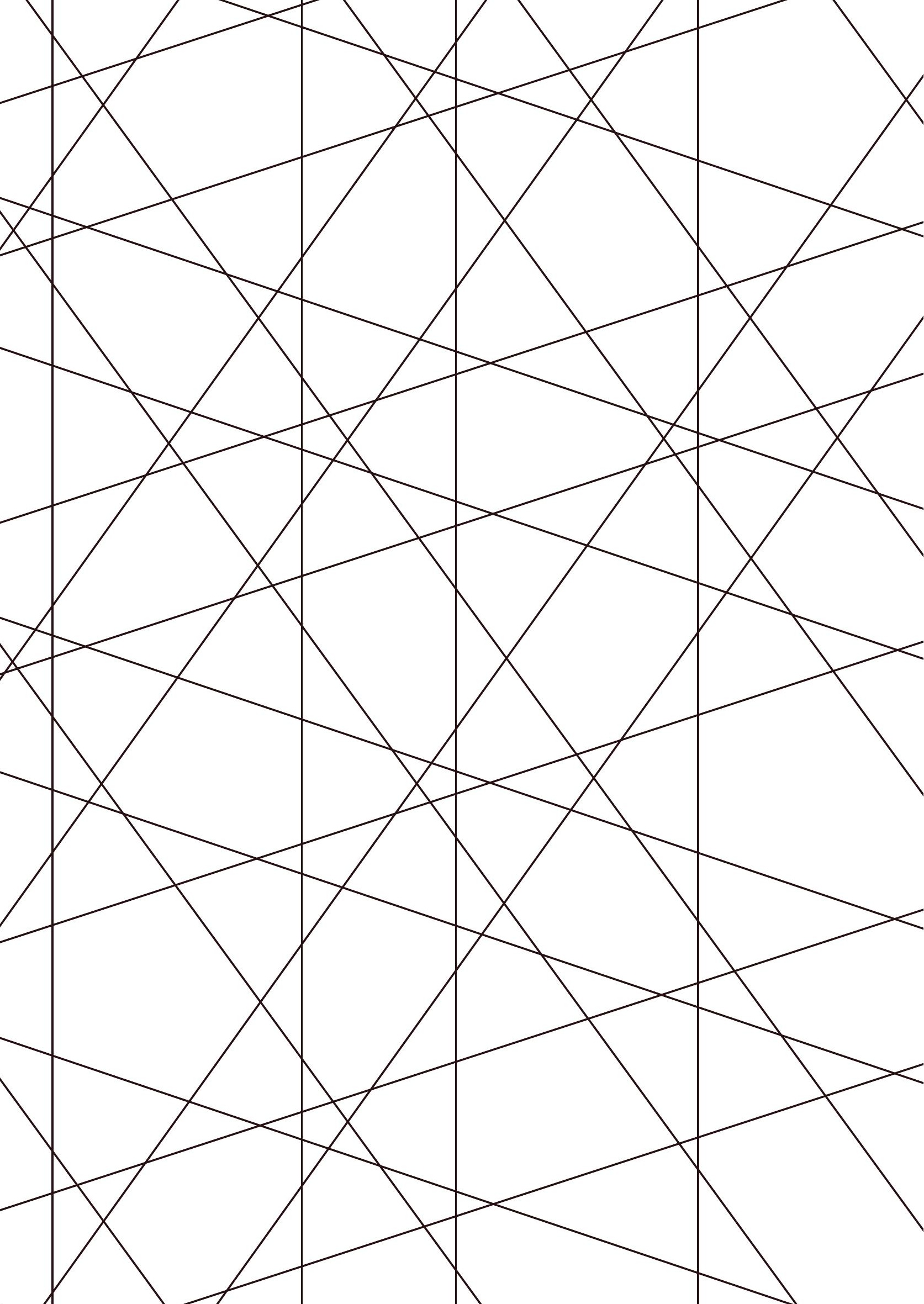


CHAPTER 1

General introduction 
The parathyroid glands were among the last anatomical discoveries and were not identified in humans until the late nineteen-hundreds by Ivar Sandström.[1] The glands are small (3-6 mm, 30-40 mg), brown, ovoid, soft structures and are generally four in number (Fig. 1).[2] Supernumerary parathyroid glands (five or more) have been reported with a prevalence of up to $14 \%$ and in approximately $3 \%$ of individuals only three glands are found.[3] Typically, the parathyroid glands are located on the posterior aspect of the thyroid gland, but ectopic glands can occur anywhere along the embryological migratory tract from the level of the mandibular angle to the pericardium.[4] The parathyroid glands play an important role in calcium homeostasis by secreting parathyroid hormone (PTH), which increases serum calcium in three ways (Fig. 2); by indirectly stimulating osteoclasts to resorb bone and release calcium into the blood, by stimulating the kidneys to reabsorb secreted calcium from the tubular fluid and by stimulating the kidneys to activate vitamin $D$, which upregulates calcium uptake from the intestine. High levels of serum calcium lead to negative feedback via calcium-sensing receptors on the parathyroid glands resulting in less secretion of PTH and normalization of serum calcium.

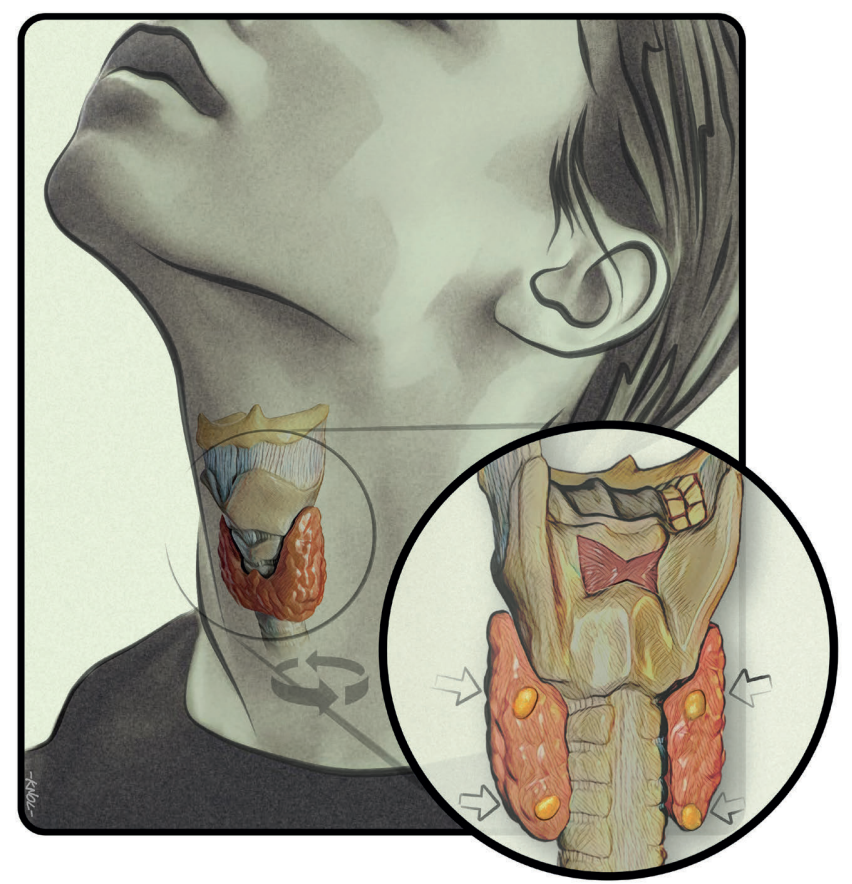

Figure 1. Anatomy of the parathyroid glands.

Posterior view of the larynx and thyroid gland with four parathyroid glands on typical locations (arrows). 


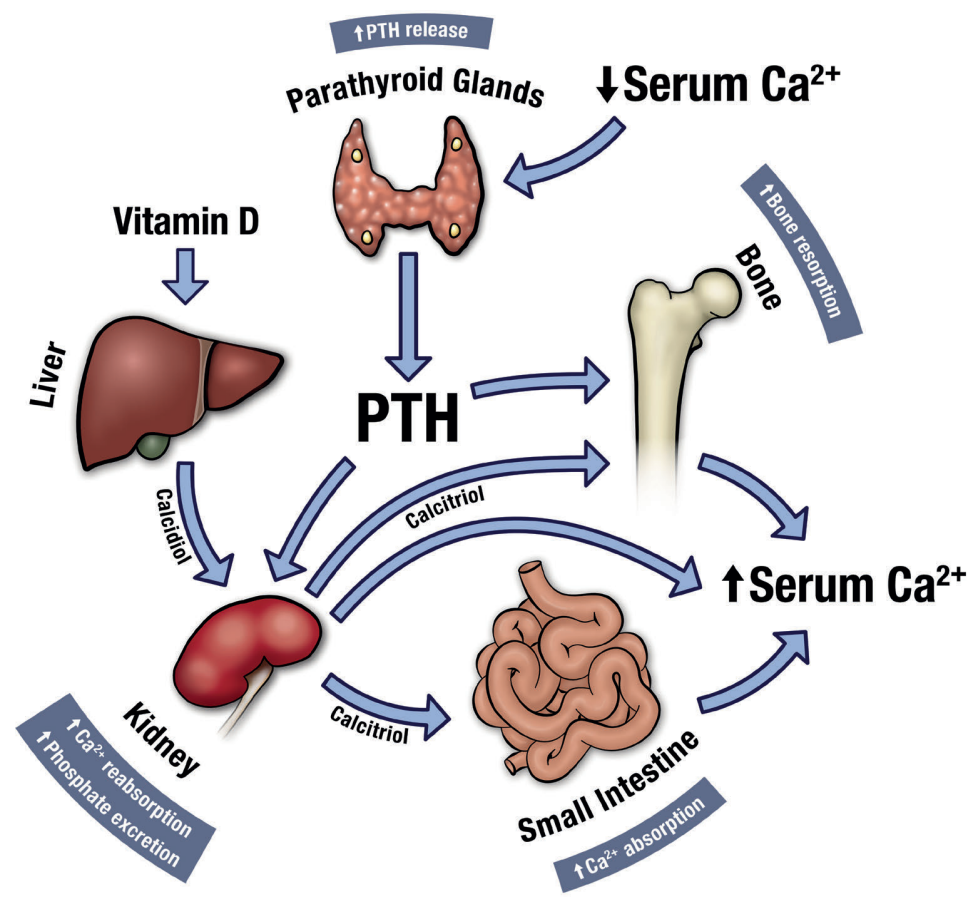

Figure 2. Calcium homeostasis.

The function of the parathyroid glands is to secrete parathyroid hormone (PTH) in response to low serum calcium $\left(\mathrm{Ca}^{2+}\right)$. Parathyroid hormone (in conjunction with vitamin D) increases serum calcium levels by interacting with bone, kidney and the small intestine.

Primary hyperparathyroidism develops as a result of autonomous production and secretion of PTH and leads to elevated serum calcium and PTH levels because of insufficient negative feedback on the parathyroid glands. The disease is most commonly caused by a solitary parathyroid adenoma (89\%), less frequently by multiglandular disease (10\%) and rarely by parathyroid carcinoma $(<1 \%)$.[5] Primary hyperparathyroidism is a relatively common endocrine disorder with a prevalence of $3 / 1000$ in the general population and up to $21 / 1000$ in postmenopausal females.[6,7] The disease is more prevalent in women with a female-to-male ratio of approximately 2.5 to 1.[8] Clinical symptoms are related to high serum calcium and PTH, including osteoporotic fractures, kidney stones, gastro-intestinal complaints and neurocognitive symptoms, classically summarized in the adage 'bones, stones, moans and groans'. [9] Secondary hyperparathyroidism typically occurs in patients with chronic renal disease due to derangements in calcium and phosphorus levels leading to excessive secretion of PTH, resulting in hyperplasia of the parathyroid glands. Tertiary hyperparathyroidism may follow after secondary hyperparathyroidism when PTH secretion transforms from compensatory to autonomous. Hyperparathyroidism can be 
treated surgically by excision of the parathyroid adenoma or by total parathyroidectomy in case of multiglandular hyperplasia. To retain parathyroid function and prevent hypoparathyroidism after total parathyroidectomy, usually a small amount of parathyroid tissue is autotransplanted into the forearm or neck, in which the autografts can be placed either intramuscularly or subcutaneously. Alternatively, a near total (31/2 gland) parathyroidectomy can be performed. Although surgical removal of an adenoma is preferred because it is the only curative therapy, pharmacological treatment with cinacalcet, a calcium-sensing receptor modulator to sensitize this receptor to the extracellular calcium, can be applied in primary hyperparathyroidism when patients are unable to undergo parathyroidectomy. Moreover, cinacalcet is progressively used to treat secondary hyperparathyroidism.[10]

In surgical treatment of hyperparathyroidism, preoperative localization of the hyperfunctioning parathyroid glands has become increasingly important, especially because of the shift from extensive bilateral neck exploration to minimally invasive parathyroidectomy in the past decades. The minimally invasive procedure shortens operative and hospital time, reduces postoperative discomfort and reduces incision length.[11,12] The current imaging method of reference is scintigraphy with ${ }^{99 \mathrm{mTC}} \mathrm{T}$ sestamibi, for which different scanning protocols (such as wash-out, pinhole or ${ }^{123}$ I-subtraction) have been investigated. There is no consensus on which scanning protocol is superior, although it is generally accepted that the highest accuracy is reached in combination with single-photon emission computed tomography / computed tomography (SPECT/CT).[13] Moreover, various radiologic methods may be used, such as ultrasonography, CT or magnetic resonance imaging (MRI).[14] A substantial number of hyperfunctioning parathyroid glands, however, remain undetected using these imaging modalities.[15]

Positron emission tomography (PET) is a nuclear medicine imaging technique in which tracers labeled with positron emitting isotopes such as ${ }^{11} \mathrm{C}$ and ${ }^{18} \mathrm{~F}$ are used (Fig. 3). Compared with conventional nuclear imaging, PET has a higher sensitivity and spatial resolution, mainly due to the concept of coincidence detection (Fig. 3).[16] Various PET tracers have been studied for parathyroid detection, but with varying results. [17] Positron-labeled choline analogues were initially observed to accumulate in parathyroid adenomas as incidental findings on PET/CT for prostate cancer imaging and have since then evolved as promising tracers for parathyroid imaging (Fig. 4).[1821] Several choline-based PET tracers are available of which ${ }^{18} \mathrm{~F}$-fluorocholine $\left({ }^{18} \mathrm{~F}\right.$ $\mathrm{FCH}$ ), also known as ${ }^{18} \mathrm{~F}$-fluoromethylcholine, is most commonly used (Fig. 5). The most important mechanism in imaging with ${ }^{18} \mathrm{~F}-\mathrm{FCH}$ is utilization of choline in cells as a 
precursor for the biosynthesis of phosphatidylcholine, an essential component of the cell membrane. After uptake of ${ }^{18} \mathrm{~F}-\mathrm{FCH}$ in the cell, it is phosphorylated by the enzyme choline kinase and is retained in the cell (Fig. 6). Other metabolic pathways of choline; oxidation to form betaine and acetylation to form acetylcholine, seem less relevant in ${ }^{18} \mathrm{~F}-\mathrm{FCH}$ imaging.[22,23]

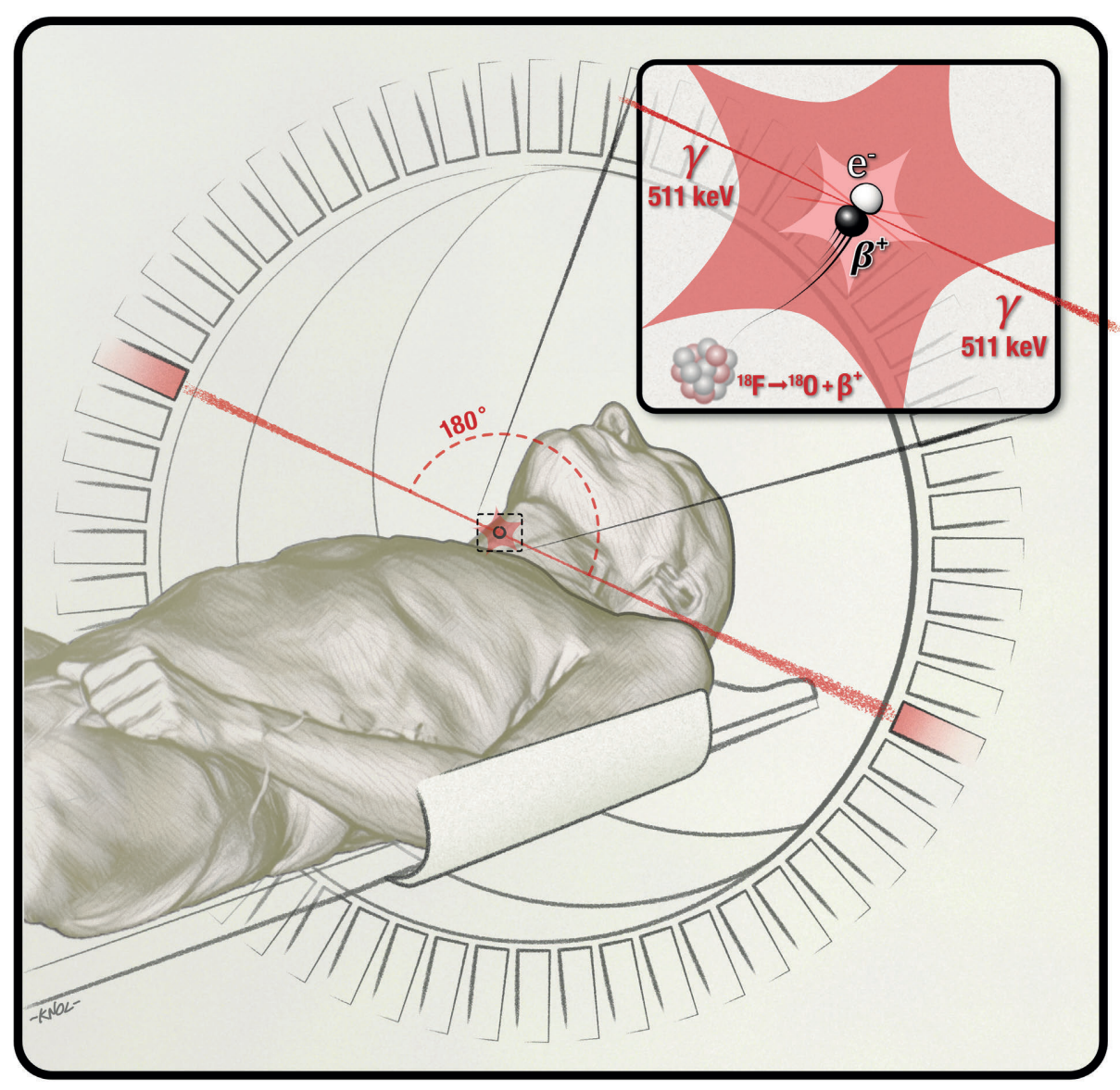

Figure 3. Positron emission tomography (PET).

In PET imaging, tracers labeled with positron-emitting radioisotopes, such as ${ }^{18} \mathrm{~F}$, are used. After decay of the isotope, a positron $\left(\beta^{+}\right)$is emitted which combines with its antiparticle, an electron (e-) and gets annihilated, all mass is converted into energy and subsequently two photons ( $y$ ) of the same energy (511 $\mathrm{keV}$ ) are emitted in opposite directions. The PET scanner detects both annihilation photons in coincidence, which enables reconstruction of tracer location. 

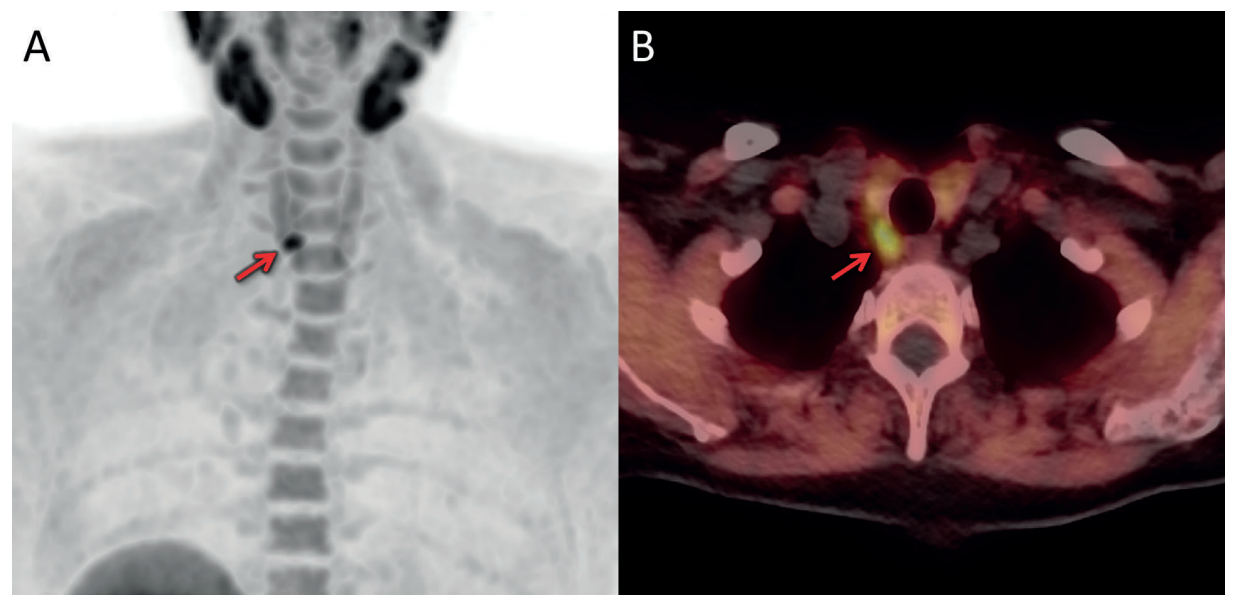

Figure 4. Example of a ${ }^{18} \mathrm{~F}$-fluorocholine PET/CT scan showing increased uptake in a right inferior parathyroid adenoma (arrow) and physiological distribution of the tracer in the salivary glands, thyroid gland, liver, muscles and bone marrow. A: PET maximum-intensity projection. B: Axial PET/CT fusion image.

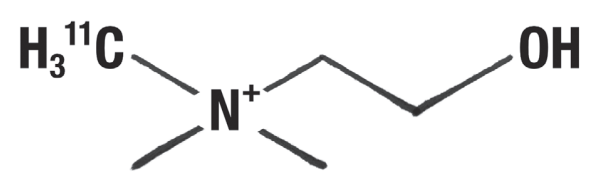

\section{[methyl-" ${ }^{11}$ ]choline (CH)}

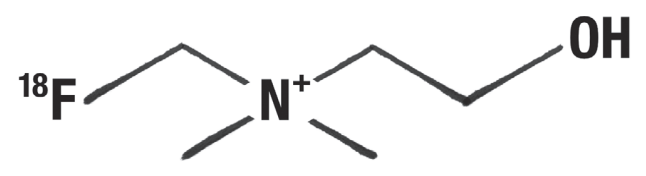

$\left[{ }^{18} \mathrm{~F}\right]$ fluorocholine (FCH)

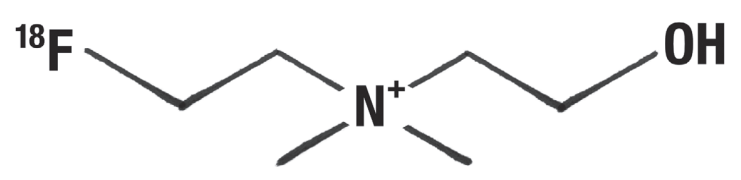

\section{$\left[{ }^{18}\right.$ F]fluoroethylcholine (FEC)}

Figure 5. Most frequently used positron-emitter labeled choline analogues. 
Since its introduction, ${ }^{18} \mathrm{~F}-\mathrm{FCH}$ PET/CT has gained general acceptance as an imaging modality for localization of hyperfunctioning parathyroid glands. Nonetheless, several unclarities still exist about the use of this novel technique. Due to the recent introduction of ${ }^{18} \mathrm{~F}-\mathrm{FCH}$ PET/CT for parathyroid imaging, the scientific literature is still relatively scarce plus the investigated patient cohorts are small and heterogeneous. Also, it is still under discussion which scan acquisition parameters should be used. ${ }^{18} \mathrm{~F}-\mathrm{FCH}$ PET/CT is regularly acquired at 60 minutes after administration of the tracer, but it is also possible to acquire additional images at multiple time points and hereby possibly increase accuracy. Moreover, it is not known what the ideal time point is to acquire early images, which could be explored using dynamic imaging. Additionally, dynamic imaging could help in distinguishing parathyroid glands from other structures with ${ }^{18} \mathrm{~F}-\mathrm{FCH}$ uptake, such as reactive lymph nodes, a common cause for false-positive scan results. Furthermore, it is unclear if ${ }^{18} \mathrm{~F}-\mathrm{FCH}$ PET/CT should only be used when conventional parathyroid imaging has been inconclusive or if it can be appropriately used as a first-line imaging modality. Finally, the distribution and incidence of additional findings on ${ }^{18} \mathrm{~F}-\mathrm{FCH}$ PET/CT is unknown in this particular patient population with hyperparathyroidism.

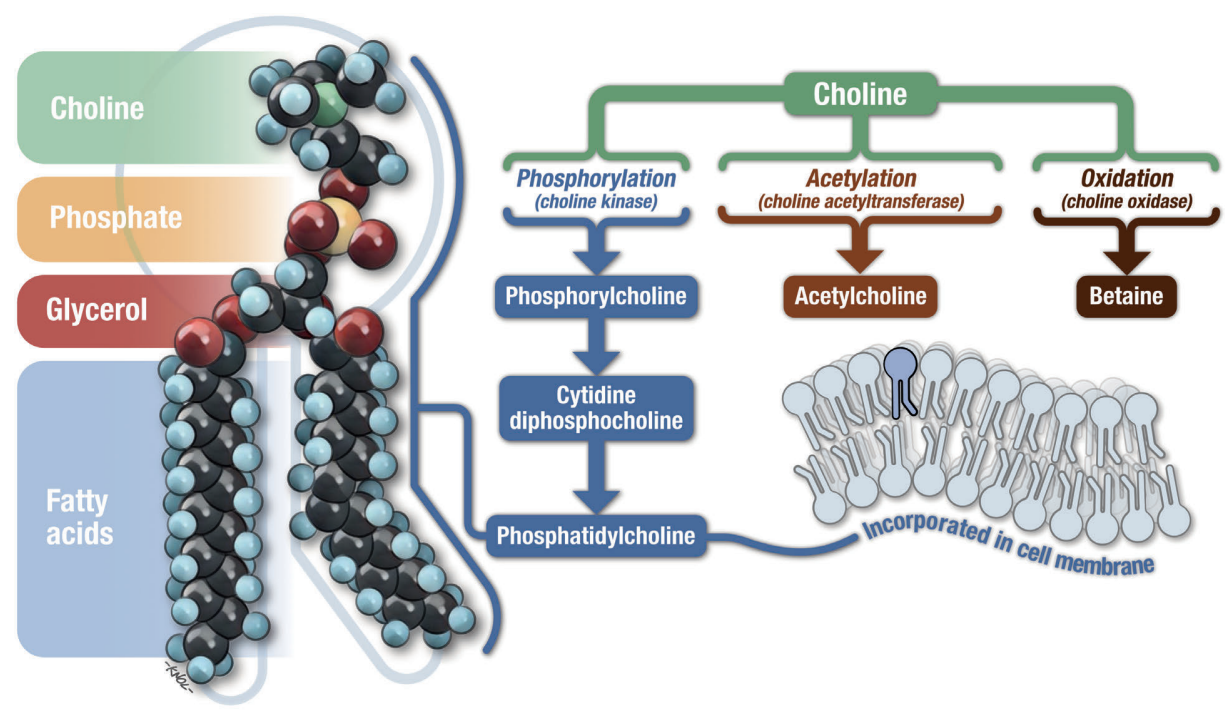

Figure 6. Cellular metabolism of choline.

Choline can be phosphorylated, acetylated and oxidized. The most important pathway in parathyroid imaging is phosphorylation. Phosphorylcholine acts as an intracellular storage pool of choline and is further converted to phosphatidylcholine, a major component of cellular membranes. 


\section{Objectives and thesis outline}

The objective of this thesis was to evaluate the use of ${ }^{18} \mathrm{~F}-\mathrm{FCH}$ PET/CT for preoperative localization of hyperfunctioning parathyroid glands in patients with hyperparathyroidism and to explore the optimal scan acquisition parameters.

\section{Part 1 - Introduction}

In addition to the concise introduction in chapter 1, chapter $\mathbf{2}$ provides a comprehensive systematic literature review on ${ }^{18} \mathrm{~F}-\mathrm{FCH}$ PET/CT for parathyroid localization, in which the current scientific literature was critically analyzed and performance data were pooled.

\section{Part 2 - Technical aspects of ${ }^{18} \mathrm{~F}$-fluorocholine PET/CT}

Different scanning protocols were evaluated in patients with histologically proven parathyroid adenomas. In chapter $\mathbf{3}$, dual-time-point scanning was studied in a cohort of 64 patients to determine the value of early image acquisition in addition to the standard imaging protocol. In chapter 4, 101 dynamic ${ }^{18} \mathrm{~F}-\mathrm{FCH}$ PET/CT scans were analyzed to assess the optimal time frame for image acquisition and to evaluate the ability of dynamic images to distinguish parathyroid adenomas from other anatomical structures with ${ }^{18} \mathrm{~F}-\mathrm{FCH}$ uptake.

\section{Intermezzo}

A case-report about a patient with tertiary hyperparathyroidism and visualization of both the hyperfunctioning parathyroid glands as well as a typical picture of renal osteodystrophy.

\section{Part 3 - Clinical aspects of ${ }^{18} \mathrm{~F}$-fluorocholine PET/CT}

In chapter 5, the use of ${ }^{18} \mathrm{~F}-\mathrm{FCH}$ PET/CT as a first-line imaging modality for localization of hyperfunctioning parathyroid glands was evaluated in a retrospective cohort study on 271 patients. In chapter 6 , the frequency and relevance of incidental findings on ${ }^{18} \mathrm{~F}$ FCH PET/CT were analyzed in a cohort of 388 patients.

\section{Part 4 - Summary, discussion and future perspectives}

The thesis is summarized in chapter 7 , a general discussion on the use of ${ }^{18} \mathrm{~F}-\mathrm{FCH}$ PET/ CT for parathyroid imaging is provided in chapter 8 and chapter 9 contains the 'impact paragraph'. 


\section{References}

1. Eknoyan G. A history of the parathyroid glands. Am J Kidney Dis 1995;26:801-807.

2. Carlson D. Parathyroid pathology: hyperparathyroidism and parathyroid tumors. Arch Pathol Lab Med 2010;134:1639-1644.

3. Lappas D, Noussios G, Anagnostis P, Adamidou F, Chatzigeorgiou A, Skandalakis P. Location, number and morphology of parathyroid glands: results from a large anatomical series. Anat Sci Int 2012;87:160164.

4. Fancy T, Gallagher D,3rd, Hornig JD. Surgical anatomy of the thyroid and parathyroid glands. Otolaryngol Clin North Am 2010;43:221-7,vii.

5. Ruda JM, Hollenbeak CS, Stack BC,Jr. A systematic review of the diagnosis and treatment of primary hyperparathyroidism from 1995 to 2003. Otolaryngol Head Neck Surg 2005;132:359-372.

6. Adami S, Marcocci C, Gatti D. Epidemiology of primary hyperparathyroidism in Europe. J Bone Miner Res 2002;17 Suppl 2:N18-23.

7. Lundgren E, Rastad J, Thrufjell E, Akerstrom G, Ljunghall S. Population-based screening for primary hyperparathyroidism with serum calcium and parathyroid hormone values in menopausal women. Surgery 1997;121:287-294.

8. Yeh MW, Ituarte PH, Zhou HC, Nishimoto S, Liu IL, Harari A, et al. Incidence and prevalence of primary hyperparathyroidism in a racially mixed population. J Clin Endocrinol Metab 2013;98:1122-1129.

9. Bilezikian JP, Bandeira L, Khan A, Cusano NE. Hyperparathyroidism. Lancet 2018;391:168-178.

10. Rroji M, Spasovski G. Calcimimetics versus parathyroidectomy: what is preferable? Int Urol Nephrol 2018;50:1271-1275.

11. Wilhelm SM, Wang TS, Ruan DT, Lee JA, Asa SL, Duh QY, et al. The American Association of Endocrine Surgeons Guidelines for Definitive Management of Primary Hyperparathyroidism. JAMA Surg 2016;151:959-968.

12. Sackett WR, Barraclough B, Reeve TS, Delbridge LW. Worldwide trends in the surgical treatment of primary hyperparathyroidism in the era of minimally invasive parathyroidectomy. Arch Surg 2002;137:1055-1059.

13. Treglia G, Sadeghi R, Schalin-Jantti C, Caldarella C, Ceriani L, Giovanella L, et al. Detection rate of ${ }^{99 \mathrm{~m} T C-}$ MIBI single photon emission computed tomography (SPECT)/CT in preoperative planning for patients with primary hyperparathyroidism: a meta-analysis. Head Neck 2016;38 Suppl 1:E2159-E2172.

14. Mihai R, Simon D, Hellman P. Imaging for primary hyperparathyroidism - an evidence-based analysis. Langenbecks Arch Surg 2009;394:765-784.

15. Hindie E, Zanotti-Fregonara P, Tabarin A, Rubello D, Morelec I, Wagner T, et al. The role of radionuclide imaging in the surgical management of primary hyperparathyroidism. J Nucl Med 2015;56:737-744.

16. Shukla AK, Kumar U. Positron emission tomography: an overview. J Med Phys 2006;31:13-21.

17. Kluijfhout WP, Pasternak JD, Drake FT, Beninato T, Gosnell JE, Shen WT, et al. Use of PET tracers for parathyroid localization: a systematic review and meta-analysis. Langenbecks Arch Surg 2016;401:925935.

18. Mapelli P, Busnardo E, Magnani P, Freschi M, Picchio M, Gianolli L, et al. Incidental finding of parathyroid adenoma with ${ }^{11} \mathrm{C}$-choline PET/CT. Clin Nucl Med 2012;37:593-595.

19. Quak E, Lheureux S, Reznik Y, Bardet S, Aide N. F18-choline, a novel PET tracer for parathyroid adenoma? J Clin Endocrinol Metab 2013;98:3111-3112. 


\section{Chapter 1}

20. Michaud L, Burgess A, Huchet V, Lefevre M, Tassart M, Ohnona J, et al. Is ${ }^{18} \mathrm{~F}$-fluorocholine-positron emission tomography/computerized tomography a new imaging tool for detecting hyperfunctioning parathyroid glands in primary or secondary hyperparathyroidism? J Clin Endocrinol Metab 2014;99:4531. 4536.

21. Lezaic L, Rep S, Sever MJ, Kocjan T, Hocevar M, Fettich J. ${ }^{18} \mathrm{~F}$-Fluorocholine PET/CT for localization of hyperfunctioning parathyroid tissue in primary hyperparathyroidism: a pilot study. Eur J Nucl Med Mol Imaging $2014 ; 41: 2083-2089$.

22. Roivainen A, Forsback S, Grönroos T, Lehikoinen P, Kähkönen M, Sutinen E, et al. Blood metabolism of [methyl-11 $\mathrm{C}$ ]choline; implications for in vivo imaging with positron emission tomography. Eur J Nucl Med 2000;27:25-32.

23. DeGrado TR, Reiman RE, Price DT, Wang S, Coleman RE. Pharmacokinetics and radiation dosimetry of ${ }^{18}$ F-fluorocholine. J Nucl Med 2002;43:92-96. 


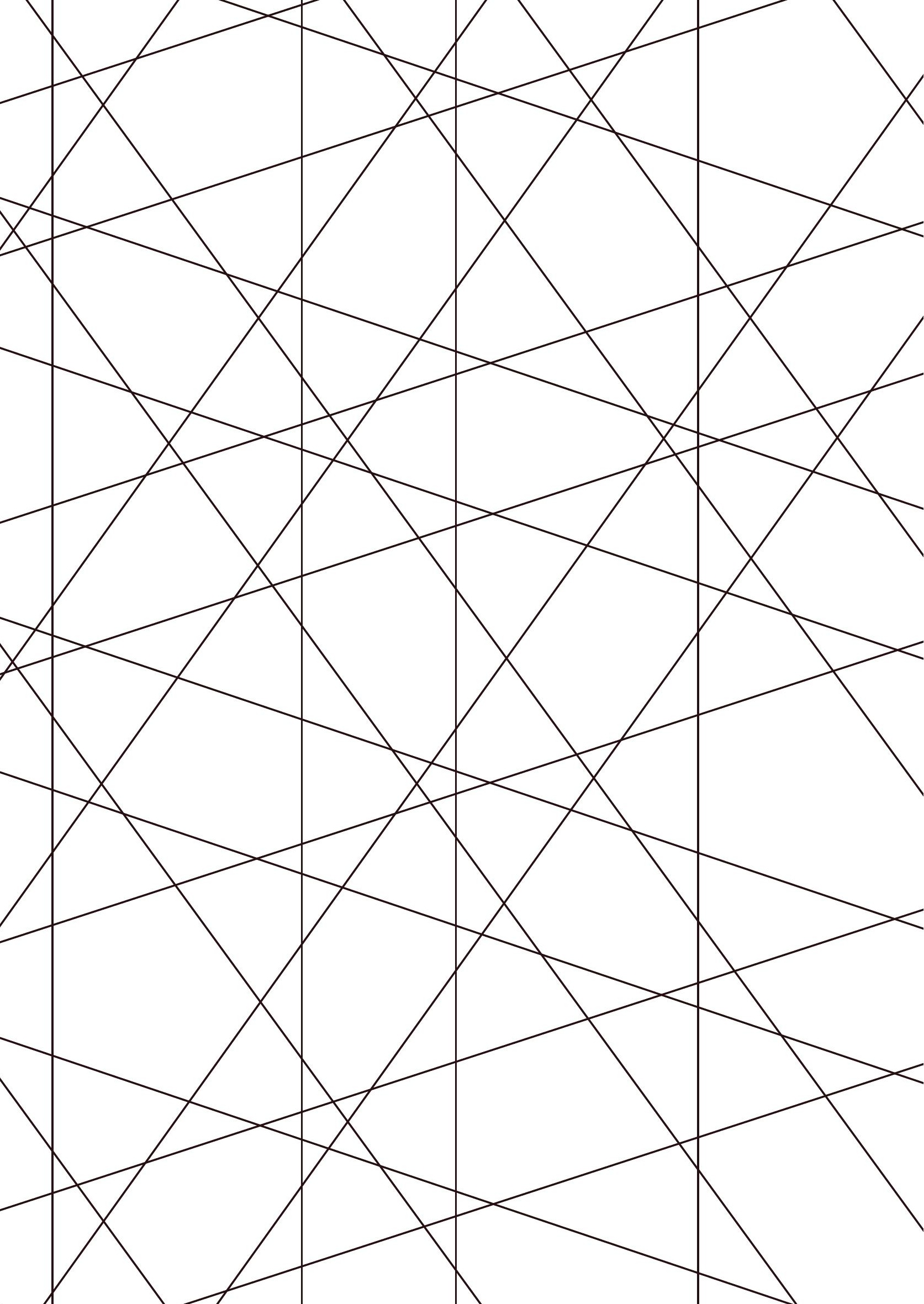




\section{CHAPTER 2}

Choline PET/CT in parathyroid imaging: a systematic review

Wouter A.M. Broos

Friso M. van der Zant

Remco J.J. Knol

Maurits Wondergem

Nuclear Medicine Communications

2019;40(2):96-10 


\section{Abstract}

One of the most promising imaging techniques in primary hyperparathyroidism is PET/ CT with choline-based tracers. To investigate the current evidence of these tracers in localizing parathyroid adenoma, a systematic review was performed. A comprehensive literature search was carried out and eligible studies were analyzed. Data were extracted, the level of evidence was scored, and performance data were pooled to calculate the weighted detection rate. Eleven articles were included in this study. The pooled detection rate was 97 and $94 \%$ on per patient-based and per lesion-based analysis, respectively. There was considerable heterogeneity between studies and the level of evidence was determined to be 3a-, following Oxford criteria. Choline PET/CT has shown favorable results in detection of hyperfunctioning parathyroid tissue and may replace conventional ${ }^{99 m} \mathrm{mc}$-sestamibi scintigraphy in preoperative planning of parathyroid surgery. However, the equality of current evidence is moderate, and additional high quality studies are needed to confirm these numbers. 


\section{Introduction}

Primary hyperparathyroidism is a common endocrine disorder, occurring with a 3/1000 prevalence in the general population and up to $21 / 1000$ in postmenopausal females. $[1,2]$ The disease develops as a result of autonomous production and secretion of parathyroid hormone (PTH) from parathyroid glands. The most common cause is a solitary parathyroid adenoma (89\%), less commonly parathyroid hyperplasia (6\%) or double parathyroid adenoma (4\%), and rarely parathyroid carcinoma (<1\%).[3] Untreated hyperparathyroidism may lead to a variety of symptoms associated with hypercalcemia and excess of circulating PTH, including decreased bone mineral density and fractures, nephrolithiasis, abdominal pain, nausea, constipation, and neuropsychiatric symptoms.

Symptomatic primary hyperparathyroidism is routinely treated with parathyroidectomy. In the past decades, surgical intervention has shifted from extensive bilateral neck exploration to minimally invasive parathyroidectomy.[4] A prerequisite for minimally invasive surgery is successful preoperative localization of the hyperfunctioning parathyroid tissue. To achieve this, various morphology-based imaging techniques can be used such as ultrasonography (US), computed tomography (CT), and MRI. However, the current reference method for localization of hyperfunctioning parathyroid tissue is functional imaging with ${ }^{99 m}$ Tc-sestamibi, which achieves detection rates of $84-88 \%$ when combined with single-photon emission computed tomography/computed tomography (SPECT/CT).[5-7]

In an attempt to further increase diagnostic performance of functional imaging for localization of parathyroid adenoma, several PET tracers have been evaluated in recent years. ${ }^{11} \mathrm{C}$-methionine PET imaging proved to perform similarly to ${ }^{99 \mathrm{mTC}} \mathrm{C}$-sestamibi but requires an on-site cyclotron for production of the radioisotope.[8] The use of the more practical and more readily available tracer ${ }^{18} \mathrm{~F}$-fluorodeoxyglucose $\left({ }^{18} \mathrm{~F}\right.$-FDG) was investigated in a few older studies, but with conflicting results and without improvement of performance compared

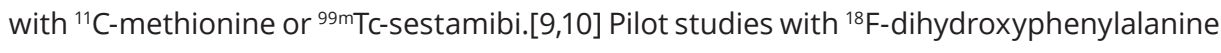
[11] or ${ }^{18} \mathrm{~F}$-fluoroethyl-L-thyrosine [12] also showed less favorable results. More recently, PET/CT with ${ }^{11} \mathrm{C}$-choline $\left({ }^{11} \mathrm{C}-\mathrm{CH}\right)$ and ${ }^{18} \mathrm{~F}$-fluorocholine $\left({ }^{18} \mathrm{~F}-\mathrm{FCH}\right)$ showed encouraging results as compared with ${ }^{99 \mathrm{~m} T c-s e s t a m i b i}$ and is therefore increasingly used.

Choline is a precursor for the biosynthesis of phospholipids, which are essential components of the cell membrane. Cells with high proliferation rate, such as tumor cells, have increased demand for choline because of enhanced phospholipid synthesis. After uptake of choline by the cell, it is phosphorylated by the enzyme choline kinase and is retained in the cell. For imaging purposes, choline can be radiolabeled with positron emitters such as ${ }^{11} \mathrm{C}$ or ${ }^{18} \mathrm{~F}$.[13] 
Choline-based PET tracers have been introduced into clinical practice for imaging of well-differentiated prostate cancer, outperforming ${ }^{18} \mathrm{~F}$-FDG.[14] Moreover, choline PET/ CT has been shown to yield positive imaging results for various nonmalignant diseases. $[15,16]$ Incidental ${ }^{11} \mathrm{C}-\mathrm{CH}$ uptake in parathyroid adenoma was initially reported by Mapelli et al.[17], and incidental uptake of ${ }^{18} \mathrm{~F}-\mathrm{FCH}$ in parathyroid adenoma was first reported by Quak et al.[18]. In clinical practice, choline PET/CT has, since then, gained general acceptance as a technique for localization of parathyroid adenoma. Nonetheless, it remains unclear whether choline PET/CT should replace conventional scintigraphy with ${ }^{99 m}$ TC-sestamibi SPECT/CT. To investigate the current evidence of the performance of choline-based tracers for localization of parathyroid adenoma, a systematic literature review was performed.

\section{Methods}

\section{Search strategy and selection criteria}

All articles reporting data on diagnostic performance of choline PET/CT in primary hyperparathyroidism were considered eligible. A comprehensive literature search was performed using the PubMed database (https://www.ncbi.nlm.nih.gov/pubmed/). Search terms included disease properties, scan type, and studied radiopharmaceutical tracer as a combination of both keywords and medical subject heading terms (Table 1). The search was updated until 15 March 2018. The following exclusion criteria were used: studies not reporting data on diagnostic performance of choline PET/CT in hyperparathyroidism, case reports, a studied population of less than five patients, review articles, editorials, and letters to the editor. No language restrictions or date limits were applied. In addition, a cross-reference check was performed by manually searching reference lists for relevant studies of all included articles, related review papers, relevant case reports, and guidelines.

\section{Data extraction and quality assessment}

Data extracted from eligible studies were author names, publication year, country of origin, patient characteristics, number of studied patients, scan indication, imaging characteristics, study design, used reference standard, and reported performance of choline PET/CT. When serum calcium and serum PTH were reported in $\mathrm{mg} / \mathrm{dL}$ or $\mathrm{pg} / \mathrm{mL}$, these values were converted to $\mathrm{mmol} / \mathrm{L}$ and $\mathrm{pmol} / \mathrm{L}$, respectively. When sensitivity or detection rates were not reported, these were calculated from the available data if possible. Scan performance was both registered on per patient-based as well as per lesion-based analysis and was extracted from the article or calculated from the study 
data if possible. The level of evidence was determined by critical appraisal of each article by two nuclear medicine physicians (W.B. and F.Z.), following the criteria for diagnostic procedures defined by the Oxford Centre for Evidence-based Medicine.[19] Disagreement was resolved in a consensus discussion with a third nuclear medicine physician (M.W.). Studies were scored on study design, representation of patient population, and whether the study could be reproduced based on the reported methods and test characteristics. Moreover, the quality of the reference standard was assessed, whether the reference standard was applied to all patients and whether the scans were read blinded from the reference standard. The patient population was considered representative in case the studied cohort was heterogeneous, had considerable size, consisted of only patients with primary hyperparathyroidism, and choline PET/CT was performed as primary diagnostic tool preoperatively. Study design was scored as either retrospective or prospective. The preferred reference standard was histopathological proof.

\section{Data analysis}

The overall performance of choline PET/CT in localizing parathyroid adenoma was evaluated by pooling collected data. Weighted detection rate was calculated on per patient-based and per lesion-based analysis.

Table 1. Search

\begin{tabular}{|c|c|}
\hline Search categories & Medline/PubMed search query \\
\hline Patient characteristics & $\begin{array}{l}\text { 1. "Parathyroid Diseases"[Mesh] OR parathyroid OR adenoma OR } \\
\text { hyperparathyroidism OR hypercalcemia OR hypercalcaemia }\end{array}$ \\
\hline \multicolumn{2}{|l|}{ Intervention } \\
\hline \multirow[t]{2}{*}{ Scan } & 2. "Positron Emission Tomography Computed Tomography"[Mesh] \\
\hline & $\begin{array}{l}\text { OR “Positron-Emission Tomography"[Mesh] OR PET OR PET/CT OR } \\
\text { positron emission tomography }\end{array}$ \\
\hline \multirow[t]{2}{*}{ Tracer } & 3. Choline OR fluorocholine OR ${ }^{18} \mathrm{~F}$-choline OR ${ }^{11} \mathrm{C}$-choline \\
\hline & Combination search: 1 AND 2 AND 3 \\
\hline
\end{tabular}




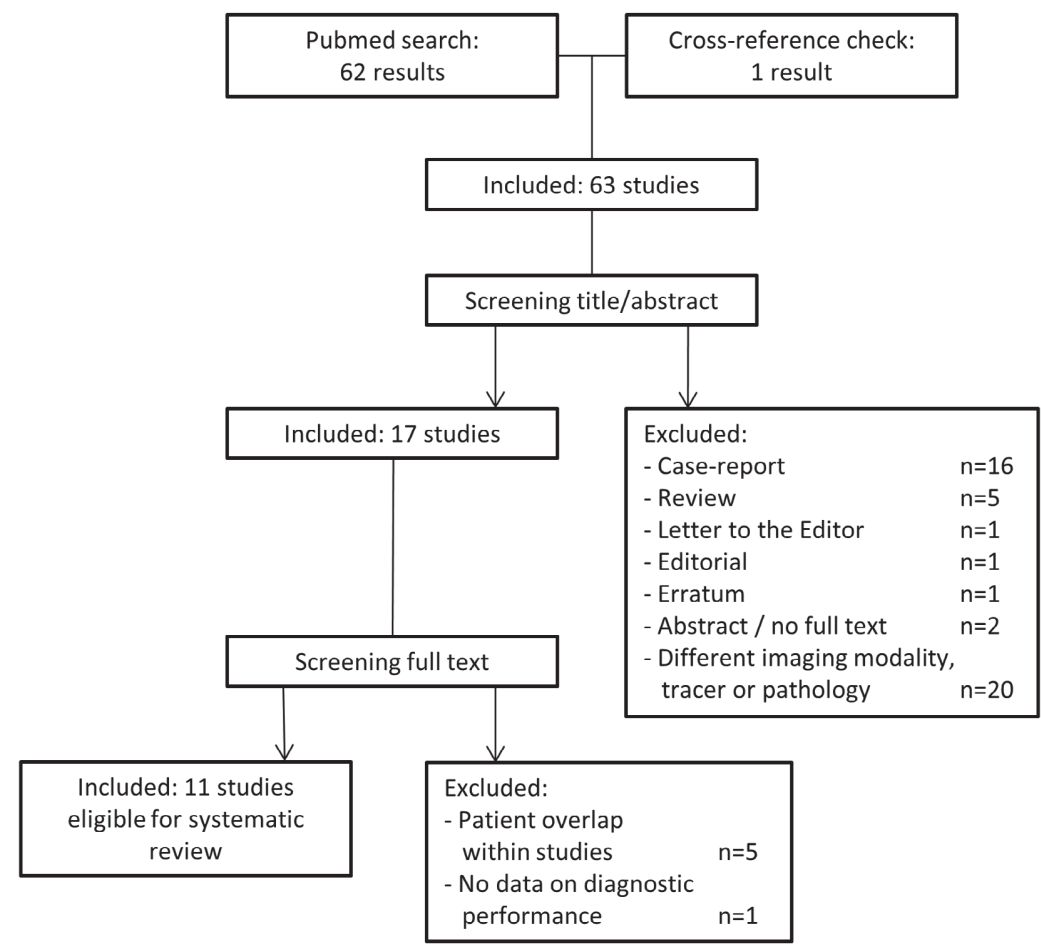

Figure 1. Flow chart for selection of eligible studies.

\section{Results}

The search resulted in 62 hits in the PubMed database. Forty-five studies were excluded following the exclusion criteria after screening the title and abstract. Cross-reference check yielded one more potential relevant study by Kubinyi et al.[20]; however, the results of this study were only presented at a congress and no full text was available, and therefore this study was excluded. Full text was screened of the remaining 15 articles. Two studies by Michaud et al.[21,22], two studies by Kluijfhout et al.[23,24], and four articles from University Medical Centre Ljubljana [25-28] had an overlap of patient cohorts, and the studies with the largest patient groups were included. One study was excluded because it lacked reporting on diagnostic performance.[29] From all searched papers, 11 studies were considered eligible for analysis (Fig. 1).

\section{Characteristics of selected studies}

The study by Orevi et al.[30] reported the preliminary results of ${ }^{11} \mathrm{C}-\mathrm{CH}$ PET/CT for localization of parathyroid adenoma in a cohort of 40 patients with hyperparathyroidism because of various etiologies. At the moment of publication, only 27 patients had 
undergone surgery. Choline PET/CT was compared with ${ }^{99 m T c-s e s t a m i b i ~ i n ~ a l l ~ p a t i e n t s . ~}$ Choline PET/CT was found to be a promising tool, providing clearer images than ${ }^{99 \mathrm{mTC}} \mathrm{C}$ sestamibi, equal accuracy with less equivocal lesions, and quicker and easier acquisition.

The selected study by Michaud et al.[22] studied ${ }^{18} \mathrm{~F}-\mathrm{FCH}$ PET/CT in 17 patients with hyperparathyroidism, also with various underlying pathology. The cohort is an extension of an earlier study published by the same author in 2014, in which 12 patients were scanned.[21] All 17 patients from that cohort underwent surgery, yielding a negative result in only one patient having lithium-induced hyperparathyroidism. Choline PET/ CT was compared with both US and ${ }^{99 m}$ Tc-sestamibi. Different approaches were studied to determine scan performance: an on-site versus a masked reading and considering equivocal images/foci as negative or positive. This pilot study confirmed that choline $\mathrm{PET/CT}$ is an adequate imaging tool in patients with hyperparathyroidism, with better sensitivity than US and not inferior to ${ }^{99 m T c-s e s t a m i b i . ~ I n t e r e s t i n g l y, ~ c h o l i n e-p o s i t i v e ~}$ thyroid lesions were detected in nine patients, among which three proved to be thyroid carcinoma.

Four eligible studies are available from the research group at University Medical Centre Ljubljana. Lezaic et al.[25] were the first to publish a patient cohort study on ${ }^{18} \mathrm{~F}-\mathrm{FCH}$ PET/CT in localizing parathyroid adenoma. The size of this cohort was 24 patients, all diagnosed with primary hyperparathyroidism. Choline PET/CT in this study was compared with ${ }^{99 \mathrm{mT}} \mathrm{T}$-sestamibi and the performance of choline PET/CT was found to be superior, particularly in patients with multiple lesions or hyperplasia. Rep et al.[26] subsequently published results of a cohort of 43 patients with primary hyperparathyroidism. The main goal of this study was to investigate optimal scan time, which was determined to be at 60 min after administration of the tracer, with highest parathyroid uptake in comparison with surrounding tissues, opposed to images at 5 or 120 min after injection. Hocevar et al.[27] retrospectively studied 151 patients with primary hyperparathyroidism, mainly focusing on surgical results, and concluded choline PET/CT is a reliable test, and patients with a single focus can safely undergo a parathyroidectomy without intraoperative PTH testing. Most recently, another study by Rep et al.[28] reported on a significantly lower radiation exposure of patients with choline PET/CT in comparison with conventional nuclear medicine imaging in a cohort of 36 patients. Because of an overlap in patient cohorts, only the study with the largest cohort, from Hocevar and colleagues, was included in the systematic review.

Kluijfhout et al.[23] published a study on ${ }^{99 m T C-s e s t a m i b i ~ i m a g i n g ~ t o ~ e n a b l e ~ m i n i m a l l y ~}$ invasive parathyroidectomy for patients with primary hyperparathyroidism, and in this 
article also their first results (five cases) of ${ }^{18} \mathrm{~F}-\mathrm{FCH}$ PET/CT are presented. In 2016, they reported results of a three-center retrospective study on ${ }^{18} \mathrm{~F}-\mathrm{FCH} \mathrm{PET/CT}$ in 43 patients with hyperparathyroidism of various etiology and inconclusive US or ${ }^{99 \mathrm{~m} T c-s e s t a m i b i .}$ [24] The scan was positive in 34 cases, and 33 patients underwent surgery. An excellent performance was found, and replacement of ${ }^{99 \mathrm{~m} T c-s e s t a m i b i}$ as a first-line imaging modality was suggested.

Taywade et al.[31] evaluated ${ }^{18} \mathrm{~F}-\mathrm{FCH}$ PET/CT in comparison with four-dimensional (4D)CT in five patients with hyperparathyroidism. The imaging modalities showed $100 \%$ concordance in the detection of parathyroid lesions, with four patients having a single lesion and one patient having three lesions.

Thanseer et al.[32] prospectively compared effectiveness of US, ${ }^{99 \mathrm{~m} T C-s e s t a m i b i, ~ a n d ~}{ }^{18} \mathrm{~F}$ FCH PET/CT in 54 patients with primary hyperparathyroidism. All lesions detected on any of the preoperative imaging techniques were surgically excised and histopathologically correlated. It was concluded that choline PET/CT was superior in accurate preoperative localization of parathyroid adenomas, especially in case of ectopic or small lesions.

Parvinian et al.[33] performed a retrospective study of $7088{ }^{11} \mathrm{C}-\mathrm{CH}$ PET/CT scans in 2933 men with prostate cancer. Thirteen patients had a choline-avid parathyroid lesion in combination with abnormally elevated serum calcium and PTH. Eight of those lesions were pathologically proven parathyroid adenomas. Incidence of parathyroid adenoma in the studied population was $0.44 \%$.

Quak et al.[34] published a prospective, bicentric study on ${ }^{18} \mathrm{~F}-\mathrm{FCH}$ PET/CT-guided surgery in patients with primary hyperparathyroidism and negative or inconclusive US and ${ }^{99 \mathrm{mTC}-}$ sestamibi scans. Twenty-four of 25 included patients underwent surgery. Choline PET/ CT guided surgery in 22 patients and bilateral cervical exploration could be avoided in $75 \%$ of patients.

Fischli et al.[35] retrospectively evaluated ${ }^{18} \mathrm{~F}-\mathrm{FCH}$ PET/CT in patients with primary hyperparathyroidism and negative or inconclusive US and ${ }^{99 \mathrm{mTC}} \mathrm{T}$-sestamibi scans. Of the total 39 included patients, 23 underwent surgery. In the subgroup of operated patients, choline PET/CT showed excellent detection and unchanged accuracy in the presence of thyroid nodules or multinodular goiters, a frequent reason of equivocal conventional imaging. 
Huber et al.[36] reported results of a retrospective study on the diagnostic value of ${ }^{18} \mathrm{~F}$ FCH PET imaging in 26 cases where US and ${ }^{99 \mathrm{mT}} \mathrm{T}$-sestamibi failed to localize parathyroid adenoma. In 11 patients, PET/CT was performed, and in 15 patients, PET/MRI. All but one of the scans detected parathyroid adenoma, all patients underwent surgery, and none of the patients needed revision surgery during follow-up.

Beheshti et al.[37] performed a prospective dual-center study in which ${ }^{18} \mathrm{~F}-\mathrm{FCH}$ PET/ CT was compared with ${ }^{99 m T c-s e s t a m i b i ~ o r ~}{ }^{99 m T c-t e t r o f o s m i n ~ S P E C T / C T . ~ O n e ~ h u n d r e d ~}$ patients were consecutively included, of which 76 underwent surgery and six received appropriate follow-up. In this study, choline PET/CT showed to be clearly superior to ${ }^{99 \mathrm{mTC}} \mathrm{C}$ sestamibi or ${ }^{99 \mathrm{mTC}} \mathrm{T}$-tetrofosmin SPECT/CT in first-line imaging of parathyroid adenomas.

A summary of the patient and imaging characteristics of the included studies can be found in Tables 2 and 3, respectively.

\section{Quality assessment of selected studies}

Each study was scored on eight items, and a level of evidence was determined following the Oxford criteria. A summary of the quality assessment is presented in Table 4.

In five studies, the patient population was considered not to be representative because only patients with negative or inconclusive results of earlier imaging received choline PET/CT.[22,24,34-36] Moreover, the small cohort of Taywade et al.[31] and the large prostate cancer cohort of Parvinian et al.[33] were considered not to be representative. Moreover, in four studies, the cohorts consisted not only of patients with primary hyperparathyroidism but also secondary, tertiary, lithium induced and with multiple endocrine neoplasia type 1.[22,24,30,36] Huber et al.[36] not only used PET/CT but also $\mathrm{PET} / \mathrm{MRI}$ in their patient cohort, and no subgroup analysis was provided for the different imaging protocols.

All studies used histopathological proof as reference standard, and in addition, Beheshti et al.[37] also used clinical follow-up. However, in some studies not all included patients underwent surgery: in Orevi et al.[30] 13/40 patients, in Kluijfhout et al.[24] 10/43 patients, in Quak et al.[34] 1/25 patients, in Fischli et al.[35] 23/39 patients, and in Beheshti et al.[37] 24/100 patients did not have surgery, and therefore, no histopathological correlation was available. Because of the different study design of Parvinian et al.[33], no histopathological correlation was provided of every patient. 


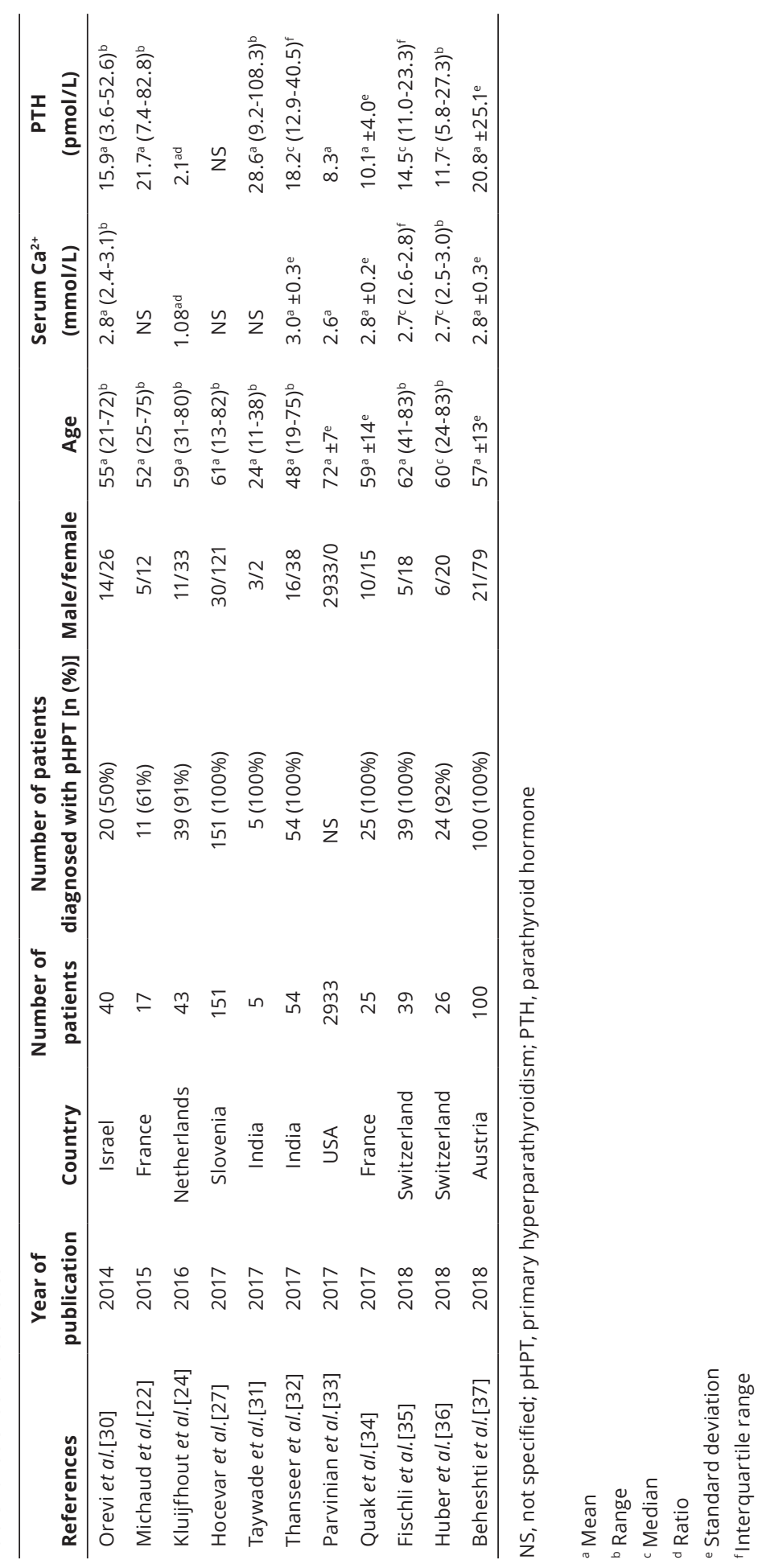




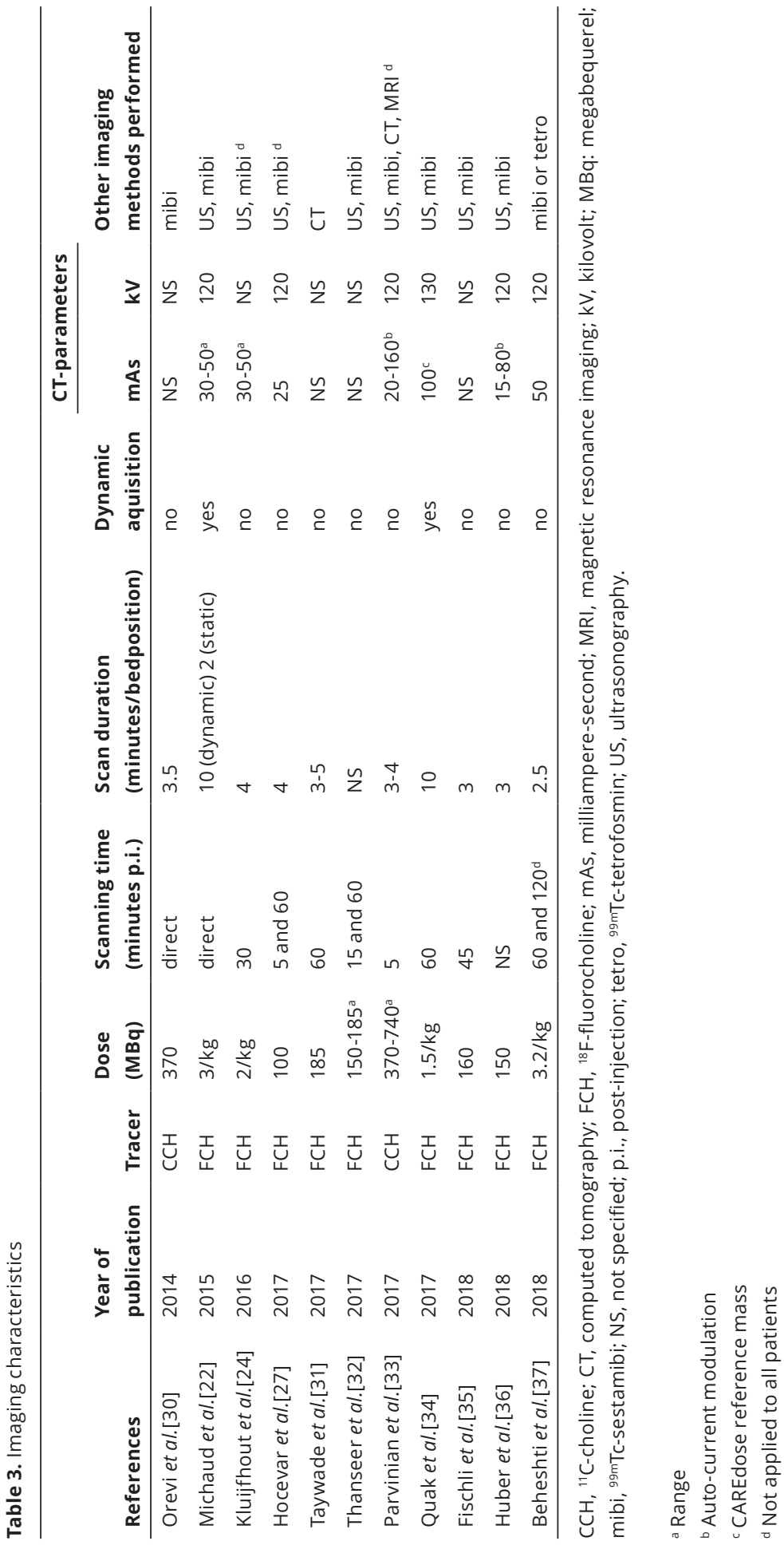


In most studies, test characteristics and study methods were clearly described and seemed reproducible; however, in the article by Huber et al.[36], the duration between administration of the tracer and time of scan acquisition was not reported. Whether patients were recruited consecutively and whether the scan was interpreted blindly was often not reported or not clear from the article.

All studies scored a level of evidence of $3 \mathrm{~b}$ or 4 . No $2 \mathrm{~b}$ or higher scores were appointed because of the lack of prospective studies with consecutively included patients or lack of consistently applied reference standards. Furthermore, none of the studies performed surgery independently of scan results. Altogether, the level of evidence that can be given to the selection of these studies is $3 a-$, with the minus sign denoting the troublesome heterogeneity between the studies (notably the variation in studied patient population).

\section{Pooled data}

For this analysis, the studies with an evidence level of 4 were excluded. An overview of total true-positives, total false-negatives, and calculated detection rate of each included study is presented in Table 5. It is to be noted that these numbers are calculated from the results of the operated patients and therefore histopathologically proven lesions only, with the exception of the cohort by Beheshti et al.[37]. In that study, the per patientbased analysis not only includes patients who underwent surgery but also six patients with negative imaging results who received appropriate clinical follow-up: three were considered false-positive and three were considered false-negative. In the study by Hocevar et al.[27], for the subgroup with two or more foci on the PET/CT, the total of removed parathyroid glands was not reported. Therefore, a per lesion-based detection rate for this study could not be calculated. Pooling of the data resulted in a detection rate of $97 \%$ in the per patient-based analysis and $94 \%$ in the per lesion-based analysis.

\section{Discussion}

In this study the current evidence of choline PET/CT for detecting hyperfunctioning parathyroid tissue was systematically reviewed. This imaging technique has only recently become in use, and all available literature on it dates from 2014 or later. One earlier systematic review is available on parathyroid adenoma localization with PET/CT, including, amongst PET with other tracers, choline PET/CT.[38] Only four studies with unique patient data were available at that time, with a total number of patients of 105 . No pooled results on performance were calculated. Data are still scarce, and published studies are very heterogeneous. Nevertheless, this present review underlines the promising capabilities of choline PET/CT to localize parathyroid adenoma, with high reported detection rates. 


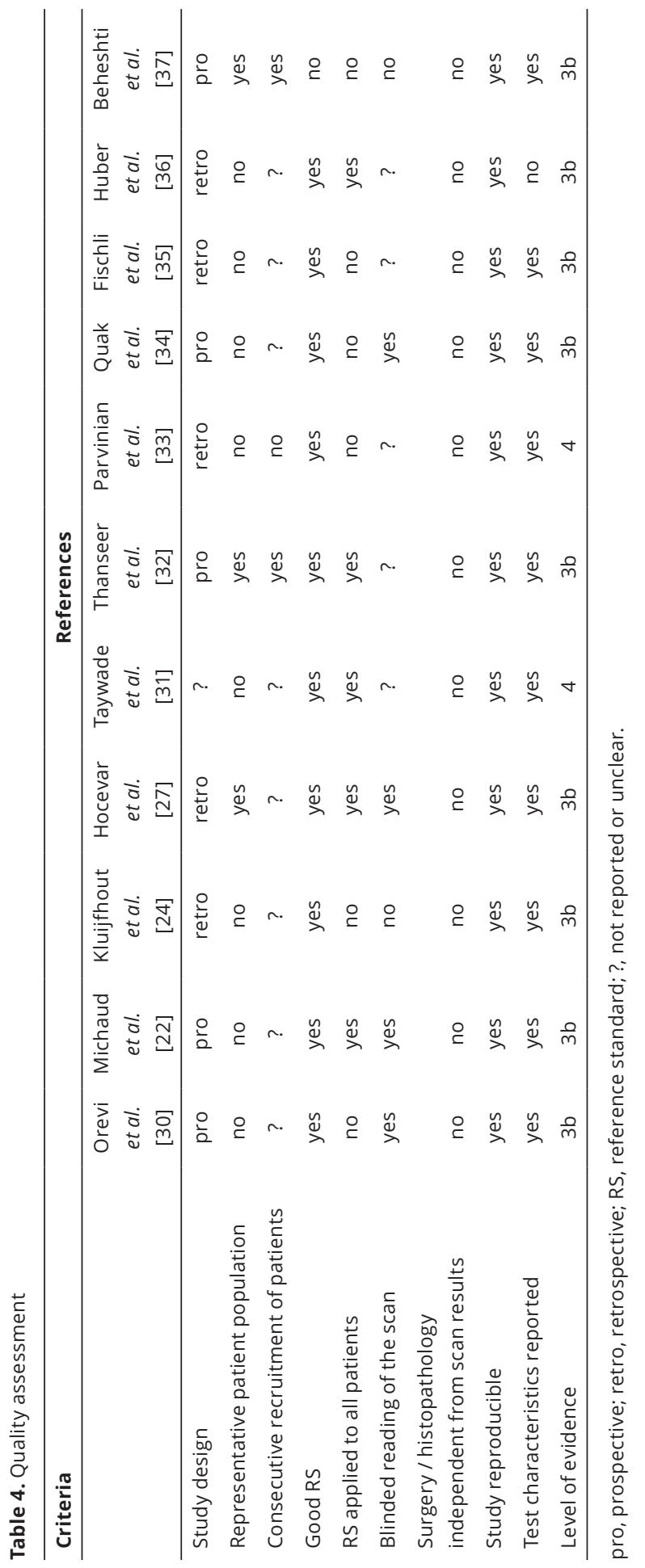


In this review, detection rate is used, calculated from the number of true-positive results divided by the total of true positives and false negatives. This is not the true sensitivity of the test because of the highly selected patient population. In essence, all selected patients with biochemically primary hyperparathyroidism have hyperfunctioning parathyroid tissue. The scans are performed to localize the disease, not to exclude parathyroid adenoma. Moreover, specificity and negative predictive value cannot reliably be calculated because of a lack of the number of true negatives. Acquiring histopathological proof of each parathyroid gland is impractical and not recommended, and impossible for ectopic parathyroid glands.[39] In addition, in most studies, the choline PET/CT was used to guide minimally invasive surgery, and inspection of contralateral glands was not performed. One study performed standard bilateral exploration independently of the scan results.[25] However, the parathyroid glands were only inspected at surgery, and no histopathological correlation was present when the glands seemed normal. Finally, except for the study by Beheshti et al.[37], studies did not report an adequate follow-up of patients to prove definite cure after parathyroidectomy.

Parathyroid imaging is mainly performed in primary hyperparathyroidism patients, in whom surgical resection of adenoma is indicated, and is performed for preoperative planning. As mentioned before, the clinical presentation of primary hyperparathyroidism is heterogeneous, and the diagnosis hyperparathyroidism is biochemical, characterized by the persistent elevation of total serum calcium levels with corresponding elevated or inappropriately normal (i.e. nonsuppressed) PTH levels.[39] Some of the analyzed studies in this review not only included primary hyperparathyroidism patients, but also secondary, tertiary, or hyperparathyroidism because of other causes. The role of

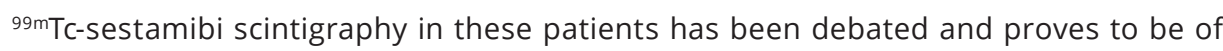
additional value in detection of supernumerary or ectopic parathyroid glands, whereas the main surgical treatment is subtotal parathyroidectomy or total parathyroidectomy with autotransplantation. $[40,41]$ The role of choline PET/CT in these diseases remains unclear but can be expected to be at least equivalent to ${ }^{99 \mathrm{~m} T c-s e s t a m i b i}$ scintigraphy.

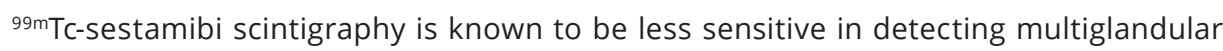
disease.[3,5] Several of the reviewed studies performed choline PET/CT in multiglandular disease. In one study, a separate analysis is reported on the performance of choline $\mathrm{PET} / \mathrm{CT}$ in solitary adenoma versus multiple adenomas or hyperplasia, however, with small patient groups.[25] A detection rate of 91\% for choline PET/CT versus 32\% for ${ }^{99 \mathrm{~m} T c-s e s t a m i b i}$ was found, compared with a detection rate of $94 \%$ for choline PET/CT in detecting solitary adenoma. In addition, the possible added value of choline PET/ CT in multiglandular disease is illustrated in a case report on the detection of a double adenoma with choline PET/CT, missed by ${ }^{99 m} \mathrm{Tc}$-sestamibi scintigraphy.[42] 


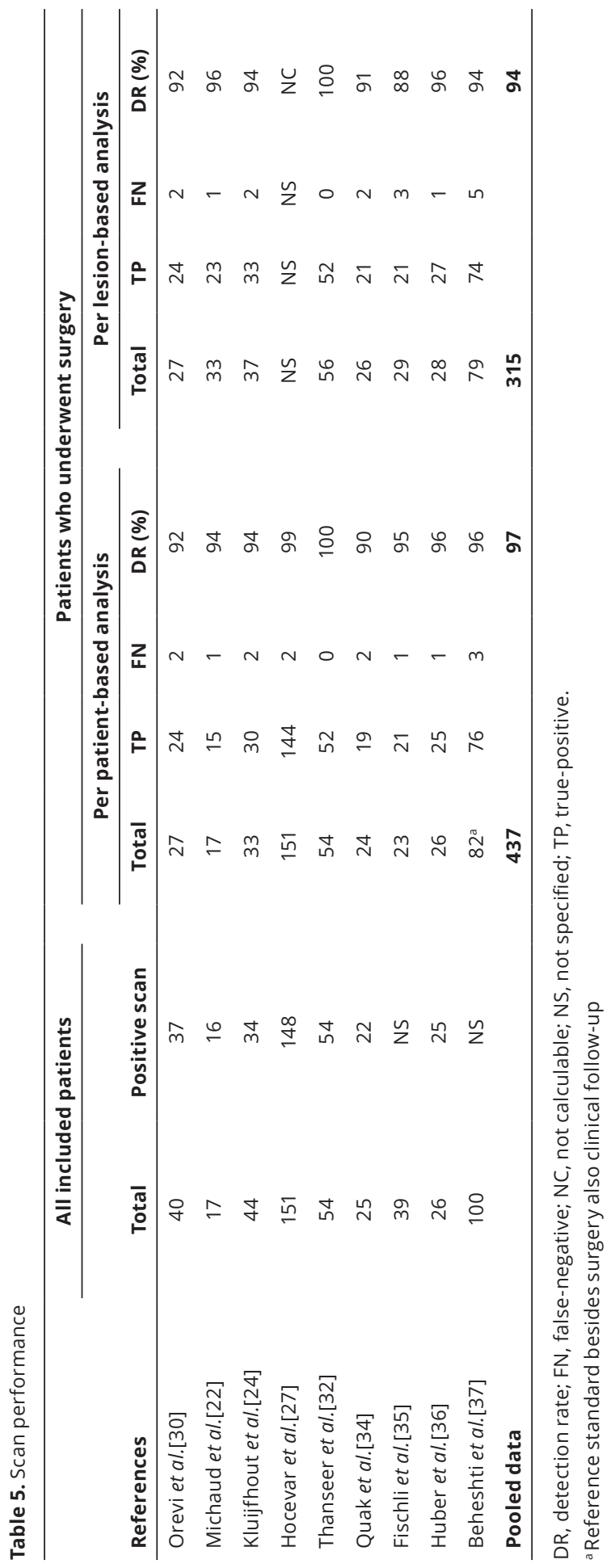


Accurate localization of a hyperfunctioning parathyroid gland allows surgeons to perform a more focused surgical approach and is mandatory for minimally invasive parathyroidectomy.[4] In all included articles, the choline PET/CT scan was used for preoperative planning and therefore was not assessed independently of surgical results, which probably introduced bias. Most studies reported both per patient-based and per lesion-based analyses of the performance. Of these, the lesion-based numbers are of more relevance for clinical practice. It is not always clear how patient-based numbers are obtained, and it is possible that a positive scan with a positive adenoma in another location was counted as a true positive, obviously introducing bias.

Ten of 11 of the reviewed studies compared choline PET/CT with ${ }^{99 m T c-s e s t a m i b i}$ scintigraphy and eight of those studies also compared choline PET/CT with US. These techniques are currently the recommended imaging modalities in primary hyperparathyroidism.[39] Historically, ${ }^{99 m T c-s e s t a m i b i ~ h a s ~ b e e n ~ t h e ~ c o n v e n t i o n a l ~}$ imaging procedure of choice in hyperparathyroidism, which can be performed using a variety of imaging protocols. There is no consensus on which technique is preferred (subtraction vs. wash-out), but SPECT/CT proved to be an important addition to planar images.[7] US is an easily accessible, noninvasive technique without radiation exposure and is the least costly; however, it cannot visualize ectopic lesions and detection rate and accuracy are lower than ${ }^{99 \mathrm{mTC}} \mathrm{C}$-sestamibi.[43,44] Conventional CT and MRI also have low detection rates.[43] Contrast-enhanced CT acquired at multiple time points (4D-CT) seems a promising technique, with high radiation dose as the main disadvantage. In a recent review, pooled sensitivity of $\mathrm{CT}$ at one time point was $71 \%, 76 \%$ for a two-phase CT, and 80\% for a three-phase CT.[45] Although MRI shows lower detection rates in comparison with $\mathrm{CT}$, combination of choline PET and MRI shows promising results with less radiation exposure.[36,46]

Choline is an nonspecific tracer for cell proliferation, and thus it also accumulates in malignancies and inflammation, which may yield false-positive results.[16,47] Several of the analyzed studies found false-positive intrathyroidal uptake, representing thyroid cancer (four cases in Michaud et al.[22], one case in Thanseer et al.[32], and an unspecified number in Parvinian et al.[33]). It must be noted that parathyroid adenoma can be localized intrathyroidal, which has earlier been reported with choline PET/CT.[48] A frequent cause of decreased performance in US and ${ }^{99 \mathrm{~m} T c-s e s t a m i b i}$ scintigraphy is benign nodular thyroid disease but this is less of a problem in choline PET/CT.[35,49,50] In the reviewed studies, no other occult malignancies are reported. 
Parathyroid adenomas may have different histopathological origins. Most adenomas are composed of mainly chief cells and a much smaller percentage is predominantly (>90\%) composed of oxyphilic cells, occurring in an estimated 3\% of cases.[51] Very rare histologic variants of parathyroid adenoma are lipoadenomas and water-clear cell adenomas.[52] Oxyphilic cells are rich in mitochondria, which probably explains the preferential ${ }^{99 \mathrm{~m} T \mathrm{~T}-s e s t a m i b i}$ retention in parathyroid lesions and the lower sensitivity of ${ }^{99 m}$ Tc-sestamibi in adenomas with a low number of oxyphilic cells.[53-55] For choline $\mathrm{PET} / \mathrm{CT}$, the difference in uptake per type of cell is unknown, and none of the reviewed studies reported histological predominant cell type.

Parathyroid hyperplasia is not, contrary to adenoma, a monoclonal proliferation. It is diffuse or multifocal expansion of the parenchymal mass of the parathyroid, involving all of the parathyroid glands to varying degrees. Choline and other tracers do not differentiate between adenoma and hyperplastic glands, and in addition, the histopathological difference is not always clear. In a metabolomics spectroscopy study, a higher concentration of choline metabolites was found in hyperfunctioning parathyroid tissue, indicating higher choline metabolism. The concentration of choline metabolites was higher in adenomas than in hyperplasia, a possible explanation for higher uptake of choline PET tracers.[56]

A rare cause of primary hyperparathyroidism is parathyroid cancer, which occurs in less than one percent of patients. Patients with parathyroid cancer are more often symptomatic. The lesions are usually larger and frequently palpable. Histologic differentiation of parathyroid can be challenging, several pathologic features can be suggestive, but local invasion of contiguous structures and lymph node or distant metastases remain the only absolute criteria for malignancy.[52,57] Parathyroid carcinoma is also known to be able to accumulate choline.[58,59] In two of the reviewed studies, a parathyroid carcinoma was found.[27,30] However, whether resection of the tumor resulted in cure of hyperparathyroidism was not reported. In one study, the lesion was negative on the choline PET/CT, and that case was excluded from analysis. [30] In the other study, it is unclear if the lesion was choline-avid, and how this case was analyzed.[27]

The major limiting factors of choline PET/CT versus conventional ${ }^{99 m}$ Tc-sestamibi scintigraphy are the number of PET centers, availability of the tracer, cost-effectiveness, and the need for more studies. No cost-effectiveness analyses are available, and costs are not discussed in the reviewed articles. In general, PET/CT is more expensive than conventional scintigraphy and SPECT/CT. However, costs can be lower if an on-site 
cyclotron is available and if a hospital functions as a referral center with frequent use of the technique. In addition, it is likely that the costs of PET/CT will further decrease, with newer PET cameras and software, requiring lower doses of PET tracers. Another disadvantage can be the availability. There are fewer centers with PET/CT scanners than with conventional gamma cameras, and a nearby cyclotron is needed for the production of the tracer. However, in the recent years, PET/CT scanners are becoming more common and the 110-min half-life of ${ }^{18} \mathrm{~F}$ makes it suitable for transportation. Practical benefits of choline PET/CT over conventional 99mTc-sestamibi SPECT/CT are shorter acquisition time, no need to stop calcimimetic drugs and lower radiation dose (2.8 mSv for dual-phase ${ }^{18} \mathrm{~F}-\mathrm{FCH}$ PET/CT vs. 6.8-7.4 mSv for ${ }^{99 \mathrm{mT}}$ Tc-sestamibi scintigraphy).[28] Future prospective studies with larger cohorts are needed to confirm the described excellent results of choline PET/CT in localizing parathyroid adenomas, and should be correlated with other upcoming detection techniques like 4D-CT and (PET/)MRI. In addition, the role of choline PET/CT in nonprimary hyperparathyroidism and in multiglandular disease can be further investigated, as well as fine-tuning the optimal scan parameters, such as acquisition technique, scanning time, and image reconstruction.

\section{Conclusion}

This systematic review indicates a high detection rate of choline PET/CT in preoperative localization of hyperfunctioning parathyroid glands in patients with primary hyperparathyroidism. However, before it can be recommended as a first-line diagnostic method, results need to be confirmed by more powerful studies, and cost-effectiveness analyses are warranted. 


\section{References}

1. Adami S, Marcocci C, Gatti D. Epidemiology of primary hyperparathyroidism in Europe.J Bone Miner Res 2002; 17 (Suppl 2):N18-N23.

2. Lundgren E, Rastad J, Thrufjell E, Akerstrom G, Ljunghall S. Population-based screening for primary hyperparathyroidism with serum calcium and parathyroid hormone values in menopausal women. Surgery 1997;121:287-294.

3. Ruda JM, Hollenbeak CS, Stack BC Jr. A systematic review of the diagnosis and treatment of primary hyperparathyroidism from 1995 to 2003. Otolaryngol Head Neck Surg 2005;132:359-372.

4. Sackett WR, Barraclough B, Reeve TS, Delbridge LW. Worldwide trends in the surgical treatment of primary hyperparathyroidism in the era of minimally invasive parathyroidectomy. Arch Surg 2002;137:1055-1059.

5. Wong KK, Fig LM, Gross MD, Dwamena BA. Parathyroid adenoma localization with ${ }^{99 m}$ Tc-sestamibi SPECT/CT: a meta-analysis. Nucl Med Commun 2015;36:363-375.

6. Wei WJ, Shen CT, Song HJ, Qiu ZL, Luo QY. Comparison of SPET/CT, SPET and planar imaging using ${ }^{99 m}$ TC-MIBI as independent techniques to support minimally invasive parathyroidectomy in primary hyperparathyroidism: a meta-analysis. Hell J Nucl Med 2015;18:127-135.

7. Treglia G, Sadeghi R, Schalin-Jantti C, Caldarella C, Ceriani L, Giovanella L, et al. Detection rate of ${ }^{99 \mathrm{~m} T C-}$ MIBI single photon emission computed tomography (SPECT)/CT in preoperative planning for patients with primary hyperparathyroidism: a meta-analysis. Head Neck 2016; 38(Suppl 1):E2159-E2172.

8. Caldarella C, Treglia G, Isgro MA, Giordano A. Diagnostic performance of positron emission tomography using ${ }^{11} \mathrm{C}$-methionine in patients with suspected parathyroid adenoma: a meta-analysis. Endocrine 2013;43:78-83.

9. Neumann DR, Esselstyn CB, Maclntyre WJ, Go RT, Obuchowski NA, Chen EQ, et al. Comparison of FDG-PET and sestamibi-SPECT in primary hyperparathyroidism. J Nucl Med 1996;37:1809-1815.

10. Chicklore S, Schulte KM, Talat N, Hubbard JG, O'Doherty M, Cook GJ. ${ }^{18} \mathrm{~F}$-FDG PET rarely provides additional information to ${ }^{11} \mathrm{C}$-methionine PET imaging in hyperparathyroidism. Clin NuCl Med 2014;39:237-242.

11. Lange-Nolde A, Zajic T, Slawik M, Brink I, Reincke M, Moser E, et al. PET with ${ }^{18} \mathrm{~F}-\mathrm{DOPA}$ in the imaging of parathyroid adenoma in patients with primary hyperparathyroidism. A pilot study. Nuklearmedizin 2006;45:193-196.

12. Krakauer M, Kjaer A, Bennedbaek FN. ${ }^{18} \mathrm{~F}-\mathrm{FET}$-PET in primary hyperparathyroidism: a pilot study. Diagnostics (Basel) 2016;6:30.

13. Vallabhajosula S. ${ }^{18} \mathrm{~F}$-labeled positron emission tomographic radiopharmaceuticals in oncology: an overview of radiochemistry and mechanisms of tumor localization. Semin Nucl Med 2007;37:400-419.

14. Jadvar H. Prostate cancer: PET with ${ }^{18} \mathrm{~F}-\mathrm{FDG},{ }^{18} \mathrm{~F}$ - or ${ }^{11} \mathrm{C}$-acetate, and ${ }^{18} \mathrm{~F}$ - or ${ }^{11} \mathrm{C}$-choline. J Nucl Med 2011;52:81-89.

15. Hodolic M, Huchet V, Balogova S, Michaud L, Kerrou K, Nataf V, et al. Incidental uptake of ${ }^{18} \mathrm{~F}$-fluorocholine $(\mathrm{FCH})$ in the head or in the neck of patients with prostate cancer. Radiol Oncol 2014;48:228-234.

16. Welle CL, Cullen EL, Peller PJ, Lowe VJ, Murphy RC, Johnson GB, et al. ${ }^{11} \mathrm{C}$-choline PET/CT in recurrent prostate cancer and nonprostatic neoplastic processes. Radiographics 2016;36:279-292.

17. Mapelli P, Busnardo E, Magnani P, Freschi M, Picchio M, Gianolli L, et al. Incidental finding of parathyroid adenoma with ${ }^{11} \mathrm{C}$-choline PET/CT. Clin Nucl Med 2012;37:593-595.

18. Quak E, Lheureux S, Reznik Y, Bardet S, Aide N. F18-choline, a novel PET tracer for parathyroid adenoma? J Clin Endocrinol Metab 2013;98:3111-3112. 
19. Howick J CEBM. Oxford Centre for Evidence-based Medicine. Levels of evidence; 2009. Available at: http://www.cebm.net/blog/2009/06/11/oxford-centre-evidence-based-medicine-levels-evidencemarch-2009/.[Accessed 19 December 2017].

20. Kubinyi J, Chroustova D, Fialovà M, Adamek S. The role of PET/CT with ${ }^{18} \mathrm{~F}$-fluorocholine in hyperparathyroidism: preliminary results in 15 patients. Eur J Nucl Med Mol Imaging 2014;41(suppl 2):S284.

21. Michaud L, Burgess A, Huchet V, Lefevre M, Tassart M, Ohnona J, et al. Is ${ }^{18} \mathrm{~F}$-fluorocholine-positron emission tomography/computerized tomography a new imaging tool for detecting hyperfunctioning parathyroid glands in primary or secondary hyperparathyroidism? J Clin Endocrinol Metab 2014;99:4531. 4536.

22. Michaud L, Balogova S, Burgess A, Ohnona J, Huchet V, Kerrou K, et al. A pilot comparison of ${ }^{18} \mathrm{~F}$-fluorocholine PET/CT, ultrasonography and ${ }^{123} /{ }^{99} \mathrm{~m} T \mathrm{~T}$-sestaMIBI dual-phase dual-isotope scintigraphy in the preoperative localization of hyperfunctioning parathyroid glands in primary or secondary hyperparathyroidism: influence of thyroid anomalies. Medicine (Baltimore) 2015;94:e1701.

23. Kluijfhout WP, Vorselaars WM, Vriens MR, Borel Rinkes IH, Valk GD, de Keizer B. Enabling minimal invasive parathyroidectomy for patients with primary hyperparathyroidism using Tc-99m-sestamibi SPECT-CT, ultrasound and first results of ${ }^{18} \mathrm{~F}$-fluorocholine PET-CT. Eur J Radio/ 2015;84:1745-1751.

24. Kluijfhout WP, Vorselaars WM, van den Berk SA, Vriens MR, Borel Rinkes IH, Valk GD, et al. Fluorine-18 fluorocholine PET-CT localizes hyperparathyroidism in patients with inconclusive conventional imaging: a multicenter study from the Netherlands. Nucl Med Commun 2016;37:1246-1252.

25. Lezaic L, Rep S, Sever MJ, Kocjan T, Hocevar M, Fettich J. ${ }^{18}$ F-Fluorocholine PET/CT for localization of hyperfunctioning parathyroid tissue in primary hyperparathyroidism: a pilot study. Eur J Nucl Med Mol Imaging 2014;41:2083-2089.

26. Rep S, Lezaic L, Kocjan T, Pfeifer M, Sever MJ, Simoncic U, et al. Optimal scan time for evaluation of parathyroid adenoma with [ $\left.{ }^{18} \mathrm{~F}\right]$-fluorocholine PET/CT. Radiol Oncol 2015;49:327-333.

27. Hocevar M, Lezaic L, Rep S, Zaletel K, Kocjan T, Sever MJ, et al. Focused parathyroidectomy without intraoperative parathormone testing is safe after pre-operative localization with ${ }^{18} \mathrm{~F}$-Fluorocholine PET/CT. Eur J Surg Oncol 2017;43:133-137.

28. Rep S, Hocevar M, Vaupotic J, Zdesar U, Zaletel K, Lezaic L. ${ }^{18} \mathrm{~F}$-choline PET/CT for parathyroid scintigraphy: significantly lower radiation exposure of patients in comparison to conventional nuclear medicine imaging approaches. J Radiol Prot 2018;38:343-356.

29. Alharbi AA, Alshehri FM, Albatly AA, Sah BR, Schmid C, Huber GF, et al. ${ }^{18} \mathrm{~F}$-Fluorocholine uptake of parathyroid adenoma is correlated with parathyroid hormone level. Mol Imaging Bio/ 2018;20:857-867.

30. Orevi M, Freedman N, Mishani E, Bocher M, Jacobson O, Krausz Y. Localization of parathyroid adenoma by ${ }^{11} \mathrm{C}$-choline PET/CT: preliminary results. Clin Nucl Med 2014;39:1033-1038.

31. Taywade SK, Damle NA, Behera A, Devasenathipathy K, Bal C, Tripathi M, et al. Comparison of ${ }^{18} \mathrm{~F}$-fluorocholine positron emission tomography/computed tomography and four-dimensional computed tomography in the preoperative localization of parathyroid adenomas-initial results. Indian J Endocrinol Metab 2017;21:399-403.

32. Thanseer N, Bhadada SK, Sood A, Mittal BR, Behera A, Gorla AKR, et al. Comparative effectiveness of ultrasonography, ${ }^{99 \mathrm{mTC}} \mathrm{T}-\mathrm{sestamibi}$, and ${ }^{18} \mathrm{~F}$-fluorocholine PET/CT in detecting parathyroid adenomas in patients with primary hyperparathyroidism. Clin Nucl Med 2017;42:e491-e497.

33. Parvinian A, Martin-Macintosh EL, Goenka AH, Durski JM, Mullan BP, Kemp BJ, et al. ${ }^{11}$ C-Choline PET/ CT for detection and localization of parathyroid adenomas. AJR Am J Roentgenol 2018;210:418-422. 
34. Quak E, Blanchard D, Houdu B, Le Roux Y, Ciappuccini R, Lireux B, et al. F18-choline PET/CT guided surgery in primary hyperparathyroidism when ultrasound and MIBI SPECT/CT are negative or inconclusive: the APACH1 study. Eur J Nucl Med Mol Imaging 2017;45:658-666.

35. Fischli S, Suter-Widmer I, Nguyen BT, Muller W, Metzger J, Strobel K, et al. The Significance of ${ }^{18} \mathrm{~F}$-fluorocholine-PET/CT as localizing imaging technique in patients with primary hyperparathyroidism and negative conventional imaging. Front Endocrinol (Lausanne) 2018;8:380.

36. Huber GF, Hullner M, Schmid C, Brunner A, Sah B, Vetter D, et al. Benefit of ${ }^{18} \mathrm{~F}$-fluorocholine PET imaging in parathyroid surgery. Eur Radiol 2018;28:2700-2707.

37. Beheshti M, Hehenwarter L, Paymani Z, RendI G, Imamovic L, Rettenbacher R, et al. ${ }^{18} \mathrm{~F}$-Fluorocholine

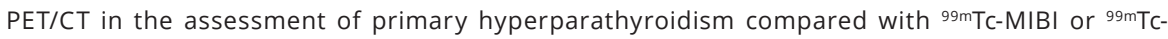
tetrofosmin SPECT/CT: a prospective dual-centre study in 100 patients. Eur J NuCl Med Mol Imaging 2018;45:1762-1771.

38. Kluijfhout WP, Pasternak JD, Drake FT, Beninato T, Gosnell JE, Shen WT, et al. Use of PET tracers for parathyroid localization: a systematic review and meta-analysis. Langenbecks Arch Surg 2016;401:925935.

39. Wilhelm SM, Wang TS, Ruan DT, Lee JA, Asa SL, Duh QY, et al. The American Association of Endocrine Surgeons Guidelines for definitive management of primary hyperparathyroidism. JAMA Surg 2016;151:959-968.

40. Karipineni F, Sahli Z, Somervell H, Mathur A, Prescott JD, Tufano RP, et al. Are preoperative sestamibi scans useful for identifying ectopic parathyroid glands in patients with expected multigland parathyroid disease? Surgery 2018;163:35-41.

41. Gasparri G, Camandona M, Bertoldo U, Sargiotto A, Papotti M, Raggio E, et al. The usefulness of preoperative dual-phase ${ }^{99 \mathrm{~m} T C}$ MIBI-scintigraphy and IO-PTH assay in the treatment of secondary and tertiary hyperparathyroidism. Ann Surg 2009;250:868-871.

42. Lalire P, Ly S, Deguelte S, Patey M, Morland D. Incremental value of ${ }^{18} \mathrm{~F}$-fluorocholine PET/CT in the localization of double parathyroid adenomas. Clin Nucl Med 2017;42:218-220.

43. Mihai R, Simon D, Hellman P. Imaging for primary hyperparathyroidism: an evidence-based analysis. Langenbecks Arch Surg 2009;394:765-784.

44. Smith RB, Evasovich M, Girod DA, Jorgensen JB, Lydiatt WM, Pagedar NA, et al. Ultrasound for localization in primary hyperparathyroidism. Otolaryngol Head Neck Surg 2013;149:366-371.

45. Kluijfhout WP, Pasternak JD, Beninato T, Drake FT, Gosnell JE, Shen WT, et al. Diagnostic performance of computed tomography for parathyroid adenoma localization; a systematic review and meta-analysis. EurJ Radiol 2017;88:117-128.

46. Kluijfhout WP, Pasternak JD, Gosnell JE, Shen WT, Duh QY, Vriens MR, et al. ${ }^{18} \mathrm{~F}$ fluorocholine PET/ MR imaging in patients with primary hyperparathyroidism and inconclusive conventional imaging: a prospective pilot study. Radiology 2017;284:460-467.

47. Burgers AMG, Wondergem M, van der Zant FM, Knol RJJ. Incidental detection of a melanoma by ${ }^{18} \mathrm{~F}$-fluorocholine PET/CT performed for evaluation of primary hyperparathyroidism. Clin NuCl Med 2018;43:265-266.

48. Paul A, Villepelet A, Lefevre M, Perie S. Oncocytic parathyroid adenoma. Eur Ann Otorhinolaryngol Head Neck Dis 2015;132:301-303.

49. Erbil Y, Barbaros U, Yanik BT, Salmaslioglu A, Tunaci M, Adalet I, et al. Impact of gland morphology and concomitant thyroid nodules on preoperative localization of parathyroid adenomas. Laryngoscope 2006;116:580-585. 


\section{Chapter 2}

50. Barbaros U, Erbil Y, Salmashoglu A, Issever H, Aral F, Tunaci M, et al. The characteristics of concomitant thyroid nodules cause false-positive ultrasonography results in primary hyperparathyroidism. $\mathrm{Am} J$ Otolaryngol 2009;30:239-243.

51. Wolpert HR, Vickery AL Jr, Wang CA. Functioning oxyphil cell adenomas of the parathyroid gland. A study of 15 cases. Am J Surg Pathol 1989;13:500-504.

52. Carlson D. Parathyroid pathology: hyperparathyroidism and parathyroid tumors. Arch Pathol Lab Med 2010;134:1639-1644.

53. Melloul M, Paz A, Koren R, Cytron S, Feinmesser R, Gal R. ${ }^{99 m}$ Tc-MIBI scintigraphy of parathyroid adenomas and its relation to tumour size and oxyphil cell abundance. Eur J Nucl Med 2001;28:209-213.

54. Westreich RW, Brandwein M, Mechanick JI, Bergman DA, Urken ML. Preoperative parathyroid localization: correlating false-negative technetium-99m sestamibi scans with parathyroid disease. Laryngoscope 2003;113:567-572.

55. Erbil Y, Kapran Y, Issever H, Barbaros U, Adalet I, Dizdaroglu F, et al. The positive effect of adenoma weight and oxyphil cell content on preoperative localization with ${ }^{99 m} \mathrm{mc}$-sestamibi scanning for primary hyperparathyroidism. Am J Surg 2008;195:34-39.

56. Battini S, Imperiale A, Taieb D, Elbayed K, Cicek AE, Sebag F, et al. High-resolution magic angle spinning ${ }^{1} \mathrm{H}$ nuclear magnetic resonance spectroscopy metabolomics of hyperfunctioning parathyroid glands. Surgery 2016;160:384-394.

57. Quinn CE, Healy J, Lebastchi AH, Brown TC, Stein JE, Prasad ML, et al. Modern experience with aggressive parathyroid tumors in a high-volume New England referral center. J Am Coll Surg 2015;220:1054-1062.

58. Deandreis D, Terroir M, Al Ghuzlan A, Berdelou A, Lacroix L, Bidault F, et al. ${ }^{18}$ Fluorocholine PET/CT in parathyroid carcinoma: a new tool for disease staging? Eur J Nucl Med Mol Imaging 2015;42:1941-1942.

59. Hatzl M, Roper-Kelmayr JC, Fellner FA, Gabriel M. ${ }^{18} \mathrm{~F}$-fluorocholine, ${ }^{18} \mathrm{~F}$-FDG, and ${ }^{18} \mathrm{~F}$-fluoroethyl tyrosine PET/CT in parathyroid cancer. Clin Nucl Med 2017;42:448-450. 


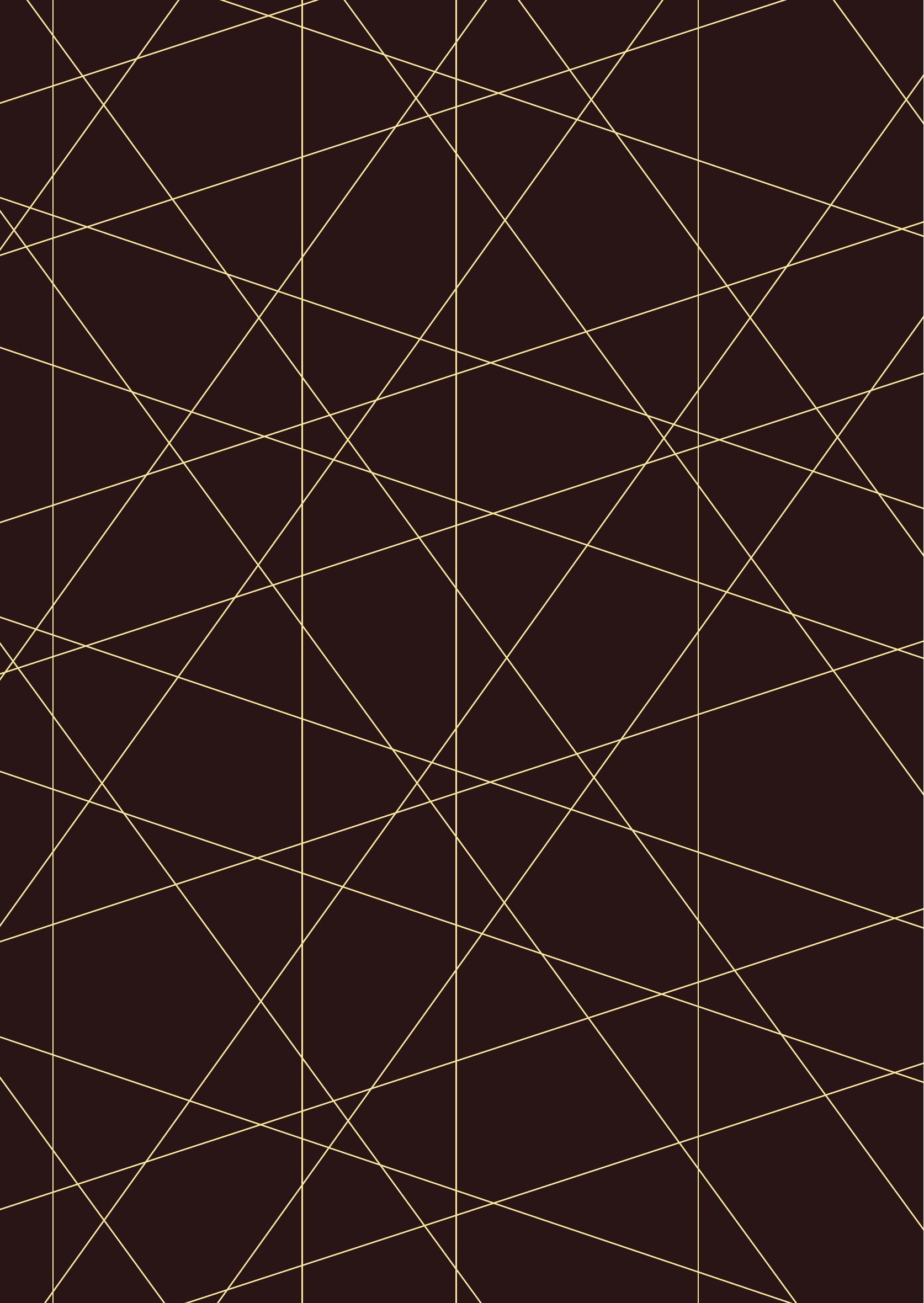




\section{PART 2}

Technical aspects of ${ }^{18} \mathrm{~F}$-fluorocholine PET/CT 


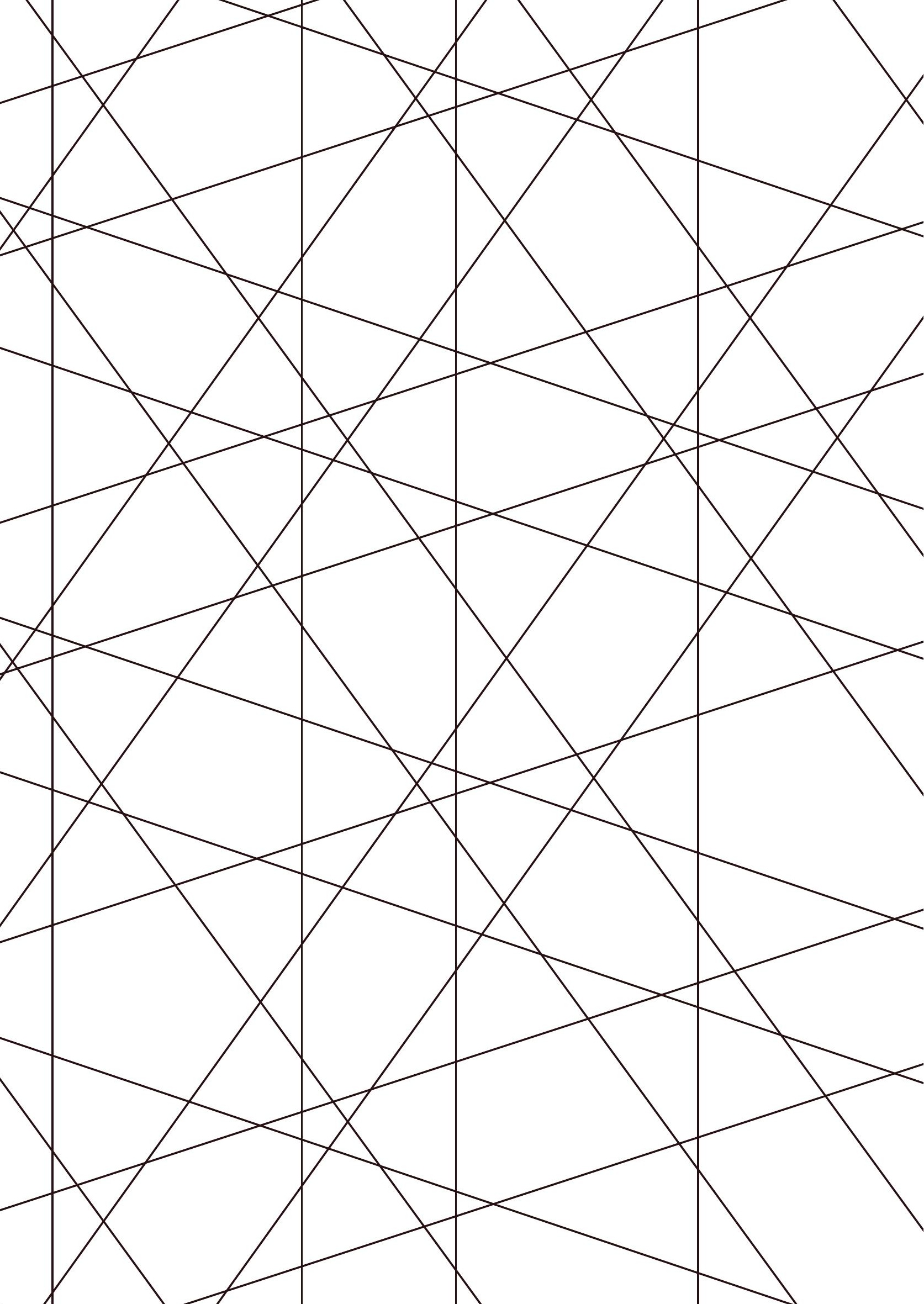




\section{CHAPTER 3 \\ Dual-time-point ${ }^{18} \mathrm{~F}$-fluorocholine PET/CT in parathyroid imaging}

Wouter A.M. Broos

Maurits Wondergem

Friso M. van der Zant

Remco J.J. Knol

Journal of Nuclear Medicine

2019;60(11):1605-1610 


\section{Abstract}

Background: ${ }^{18} \mathrm{~F}$-fluorocholine $\left({ }^{18} \mathrm{~F}-\mathrm{FCH}\right) \mathrm{PET} / \mathrm{CT}$ is a promising and increasingly used scan technique in the preoperative imaging of parathyroid adenoma. Several acquisition methods have been evaluated in the literature, but the optimal image acquisition time point after administration of the tracer is still under debate.

Methods: Patients who had hyperparathyroidism, underwent dual-time-point ${ }^{18} \mathrm{~F}-\mathrm{FCH}$ PET/CT (image acquisition, 5 min; 60 min after injection), and had histologically proven pathologic parathyroid glands were retrospectively included in the study. Early and late images were compared both visually and quantitatively.

Results: Sixty-four patients were included, and a total of 71 parathyroid glands were surgically removed. Visually, there were no differences between early and late images of hyperfunctioning parathyroid glands in 44 patients (69\%); in 13 patients (20\%), visualization on early images was better; in 6 patients (9\%), visualization of hyperfunctioning glands was best on late images; and in 1 patient (2\%), the lesion was exclusively visualized on late images. For the total cohort, there was a significant decrease in ${ }^{18} \mathrm{~F}-\mathrm{FCH}$ uptake in the glands on late versus early time points $(P=0.001)$, but there was a significant increase in the ratio of parathyroid uptake to thyroid uptake $(P=0.037)$. The group of patients with better visualization on early images showed a decrease over time in both parathyroid uptake and the ratio of parathyroid uptake to thyroid uptake, significant in comparison to those in both the group with better visualization at later time points and the group in which visualization was similar at both time points ( $P$ values of $0.000-0.018$ ). There were no significant differences in ${ }^{18} \mathrm{~F}$ $\mathrm{FCH}$ uptake and the ratio of parathyroid uptake to thyroid uptake between the latter 2 groups ( $P$ values of $0.200-0.709$ ).

Conclusion: In most patients (89\%), hyperfunctioning parathyroid glands were adequately visualized on early imaging; however, in a subset of patients (11\%), such glands were best visualized at later time points. Therefore, we recommend the acquisition of dualtime-point images in parathyroid imaging with ${ }^{18} \mathrm{~F}-\mathrm{FCH}$ PET/CT or the creation of an opportunity to acquire additional late images after review of early images when findings are inconclusive. 


\section{Introduction}

Hyperparathyroidism covers a spectrum of parathyroid diseases, with primary hyperparathyroidism being the most common. This disease develops as a result of the autonomous production and secretion of parathyroid hormone (PTH) from the parathyroid glands, with prevalences of 3 in 1000 in the general population and up to 21 in 1000 in postmenopausal women.[1,2] Secondary hyperparathyroidism typically occurs in patients with chronic renal disease because derangements in calcium and phosphorus levels result in increased PTH. Tertiary hyperparathyroidism is the condition that may follow after secondary hyperparathyroidism when PTH secretion transforms from compensatory to autonomous.

Symptomatic primary hyperparathyroidism is routinely treated with parathyroidectomy, in which the accurate localization of hyperfunctioning parathyroid glands can be beneficial in preoperative planning and is a prerequisite for minimally invasive surgery. In secondary and tertiary hyperparathyroidism, which also may be treated with parathyroidectomy, imaging is mainly performed to detect ectopic or supernumerary glands.[3] To visualize parathyroid lesions, various morphology-based imaging techniques, such as ultrasonography, CT, and MRI, can be used; however, the current reference method is functional imaging with ${ }^{99 \mathrm{~m} T c-s e s t a m i b i . ~ R e c e n t l y, ~}{ }^{18} \mathrm{~F}$-fluorocholine $\left({ }^{18} \mathrm{~F}-\mathrm{FCH}\right) \mathrm{PET} / \mathrm{CT}$ was proposed and investigated as a tool for the detection of hyperfunctioning parathyroid tissue, with promising results.[4,5]

Although various acquisition protocols have been described, the optimal time point for image acquisition after injection of ${ }^{18} \mathrm{~F}-\mathrm{FCH}$ is still under debate. In previous studies, time points ranging from 0 to 120 min after injection were used, with conflicting results. Scanning at both early and late time points has been suggested, and authors have reported lesions that were visualized on early images only.[6-8] The objective of the present study was to evaluate different uptake patterns and differences in the visualization of parathyroid glands on early and late images using dual-timepoint ${ }^{18} \mathrm{~F}-\mathrm{FCH}$ $\mathrm{PET/CT}$ in patients with hyperparathyroidism. 


\section{Methods}

\section{Patient selection}

Patients who underwent dual-time-point ${ }^{18} \mathrm{~F}-\mathrm{FCH}$ PET/CT for the evaluation of hyperparathyroidism were included in this study when both PET/CT results were positive and PET/CT was followed by surgery and when parathyroid adenoma or hyperplasia was histopathologically proven. All patient data, including baseline characteristics, scan results, and quantitative measurements, were prospectively entered into a database. Additional follow-up data were retrieved from the electronic patient records. Patients were classified on the basis of clinical indication: primary, secondary, or tertiary hyperparathyroidism. Primary hyperparathyroidism was defined as either a persistent elevation of serum calcium levels with corresponding elevated or nonsuppressed PTH levels or high PTH levels and normal serum calcium levels (i.e., normocalcemic hyperparathyroidism).[9] Secondary hyperparathyroidism was defined as low serum calcium levels and high PTH levels associated with chronic renal failure, and tertiary hyperparathyroidism was defined as a persistent elevation of PTH levels after treatment of secondary hyperparathyroidism because of autonomous PTH production.

\section{Informed consent}

The institutional research department approved this retrospective study, and all patients signed written informed consent for the use of their anonymized data for scientific purposes. Besides the standard imaging protocol and clinical management, no additional measurements or actions affecting the patient were performed. The study was performed in accordance with the Declaration of Helsinki. Approval of the local ethics committee was not necessary for the present study because the study did not fall within the scope of the Dutch Medical Research Involving Human Subjects Act (section 1.b WMO; February 26, 1998).

\section{Scan acquisition}

PET/CT images were acquired on a Siemens Biograph-16 True-Point PET/CT camera (Siemens Healthcare). Early images of the thyroid region were acquired 5 min after intravenous injection of approximately $150 \mathrm{MBq}$ of ${ }^{18} \mathrm{~F}-\mathrm{FCH}$. The following acquisition parameters were used: 1 bed position for $480 \mathrm{~s}$, a matrix size of $256 \times 256$, low-dose CT for attenuation correction, a tube current of $40 \mathrm{mAs}$ at $100 \mathrm{kV}$, Siemens CARE Dose 4D dose modulation, collimation of $24 \times 1.2 \mathrm{~mm}$, and a pitch of 0.95 . Late images ranging from the temporomandibular joint to the diaphragm were acquired $60 \mathrm{~min}$ after injection. Corresponding with early images, late images were acquired at $480 \mathrm{~s}$ per bed position and a matrix size of $256 \times 256$. Late CT images for attenuation correction and anatomic 
mapping were acquired using a tube current of $80 \mathrm{mAs}$ at $120 \mathrm{kV}$, collimation of $16 \times 1.2$ $\mathrm{mm}$, and a pitch of 0.95 . Both early and late PET images were reconstructed with an iterative 3-dimensional method using 5 iterations, 8 subsets, and a gaussian filter. For interpretation of the images and quantitative measurements, dedicated software (Syngo.via; Siemens Medical Solutions) was used. Patient preparation consisted of hydration with $1 \mathrm{~L}$ of water and, if applicable, discontinuation of colchicine $48 \mathrm{~h}$ before ${ }^{18} \mathrm{~F}-\mathrm{FCH}$ administration. Discontinuation of calcimimetic drugs or other medications was not required.

\section{Image interpretation}

Imaging results were prospectively recorded in a database after scan interpretation. The results of each ${ }^{18} \mathrm{~F}-\mathrm{FCH}$ PET/CT scan were scored as negative, equivocal, or positive for hyperfunctioning parathyroid glands. In the presence of well-defined focal ${ }^{18} \mathrm{~F}-\mathrm{FCH}$ uptake at typical locations, the scan results were considered positive for hyperfunctioning parathyroid tissue. If tracer uptake was of very low intensity or could not be distinguished from uptake in the thyroid gland, then the scan results were considered equivocal. $A$ physiologic distribution of ${ }^{18} \mathrm{~F}-\mathrm{FCH}$ or an abnormal distribution with an explanation other than hyperfunctioning parathyroid tissue defined the scan results as negative.

Other image data that were entered into the database included the locations of abnormal foci (left or right, cranial or caudal with respect to the thyroid gland, intrathyroidal or ectopic) and the numbers of abnormal foci (solitary adenoma, multiple adenomas, or 4-gland hyperplasia). Early and late images were compared both quantitatively and visually. Visually, the scans were compared independently by 2 nuclear medicine physicians. Each scan was categorized into 1 of 5 subcategories: visualization on early images only, best visualization on early images, no essential difference, best visualization on late images, and visualization on late images only. In case of a discrepancy between the 2 readings, the appropriate category was assigned by consensus. Acquired quantitative measurements of abnormal foci were SUV $V_{\text {max }}$ and SUV $V_{\text {peak }}$. Additionally, the SUVs of the thyroid gland were measured as a reference, and the ratios of parathyroid uptake to thyroid uptake (P/T ratios) were calculated.

\section{Statistical analysis}

Normally distributed continuous data were expressed as mean \pm SD and range, and nonnormal continuous data were expressed as median and interquartile range. Noncontinuous data were expressed as numbers and percentages. A paired-samples $t$ test or a Mann-Whitney $U$ test was used when appropriate to compare differences between early and late time points and between subgroups. A $P$ value of less than 0.05 was considered significant. Statistical analysis was performed using the Statistical Package for the Social Sciences, version 20 (IBM SPSS Statistics). 


\section{Results}

Sixty-four patients were included in the study, and ${ }^{18} \mathrm{~F}$-FCH scans were performed from April 28, 2016, to March 14, 2018 (Table 1). Clinical indications for ${ }^{18} \mathrm{~F}-\mathrm{FCH}$ PET/CT were primary hyperparathyroidism (including multiple endocrine neoplasia type 2a-associated disease and lithium-induced disease) in 61 patients, secondary hyperparathyroidism in 2 patients, and tertiary hyperparathyroidism in 1 patient. A total of 71 glands were surgically removed. The locations of the removed glands were as follows: 9 (13\%) left superior, 25 (35\%) left inferior, 6 (9\%) right superior, and 27 (38\%) right inferior; 1 gland (1\%) was located within the thyroid gland; and 3 glands (4\%) were ectopically located. Histopathologic examination yielded 59 single adenomas and 2 double adenomas, concordant with the scan results. For 1 patient with secondary hyperparathyroidism, 4-gland hyperplasia was shown on the scan; 3 of the excised glands were histologically confirmed to be hyperplastic; and the fourth gland was not found and was left in situ. The scan of the other patient with clinically suspected secondary hyperparathyroidism showed single-gland disease (recurrent hyperparathyroidism after earlier excision of a contralateral parathyroid adenoma). For the patient with tertiary hyperparathyroidism, 4-gland hyperplasia was also shown on the scan; all of the glands were removed and histopathologically confirmed to be hyperplastic. All patients showed a clinically significant decrease in PTH levels after surgery (at least a 50\% reduction).

Table 1. Patient characteristics

\begin{tabular}{lc}
\hline Age [mean \pm SD (range)] (years) & $60 \pm 12(28-81)$ \\
Sex [n (\%)] & $17(27)$ \\
$\quad$ Male & $47(73)$ \\
Female & $21.0 \pm 22.3(5.3-171.2)$ \\
PTH [mean \pm SD (range)] (pmol/L) & \\
$\quad$ (normal range: $1.3-6.8 \mathrm{pmol} / \mathrm{L})$ & $2.67 \pm 0.19(2.11-3.30)$ \\
\hline$\quad$ (normal range: $2.10-2.50 \mathrm{mmol} / \mathrm{L})$ &
\end{tabular}

In patients with multiple pathologic parathyroid glands shown on ${ }^{18} \mathrm{~F}-\mathrm{FCH}$ PET/CT, 1 gland was randomly selected for further analysis to avoid potential bias due to nonindependent observations, resulting in a total of 64 analyzed lesions. Visual comparison yielded no essential difference in the visibility of the pathologic parathyroid gland on early and late images from 44 scans (69\%). Thirteen scans (20\%) showed better visualization on early images, and 6 scans (9\%) showed better visualization on late images. One scan (2\%) had negative results on early images but showed a single parathyroid adenoma on late images, whereas no scans had exclusively positive results on early images. Examples of best visualization on early and late images are shown in Figures 1 and 2, respectively. 

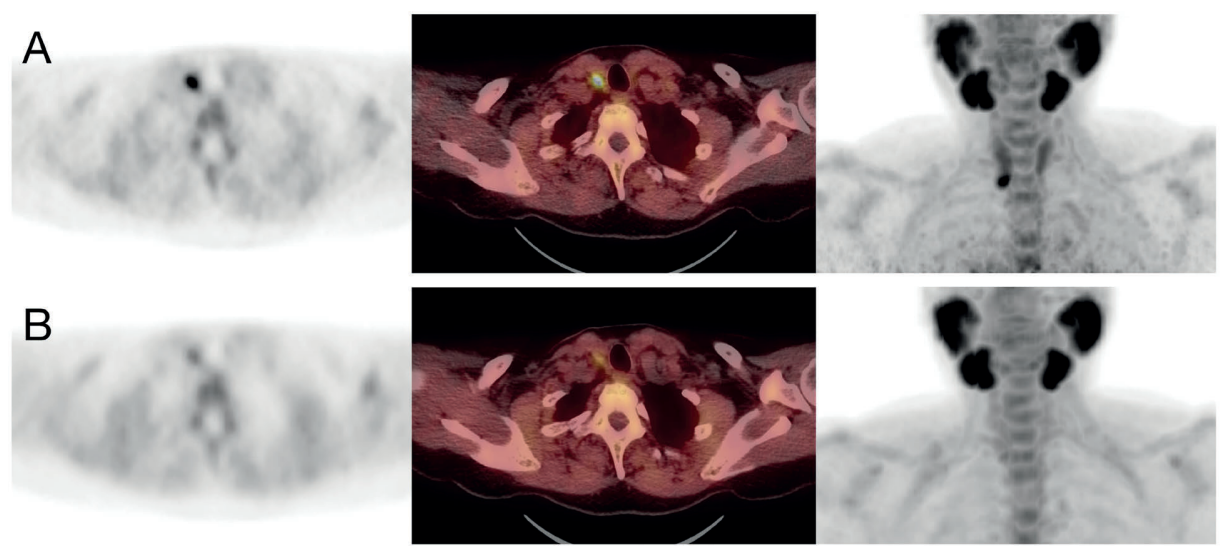

Figure 1. Example of dual-time-point ${ }^{18} \mathrm{~F}$-fluorocholine PET/CT with best visualization of parathyroid adenoma on early images. (A) Early images (from left to right: attenuation-corrected [AC] PET, PET/CT fusion, and maximum-intensity projection [MIP] images acquired at $5 \mathrm{~min}$ after injection) show intense tracer uptake in right inferior parathyroid adenoma. (B) Late images (from left to right: AC PET, PET/CT fusion, and MIP images at 60 min after injection) show nearly complete washout of tracer.
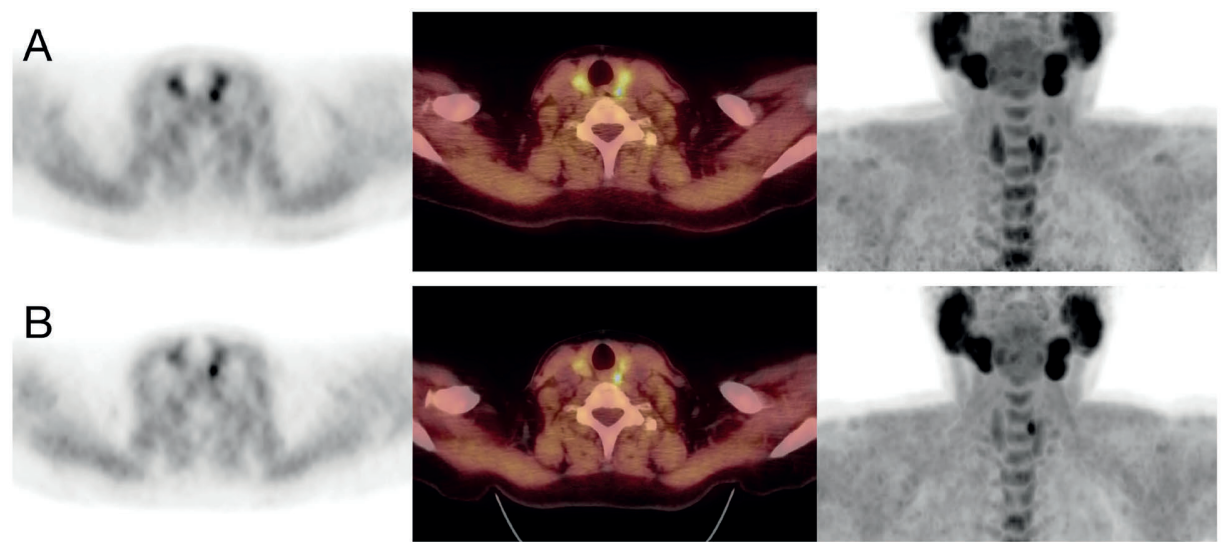

Figure 2. Example of dual-time-point ${ }^{18} \mathrm{~F}$-Fluorocholine PET/CT with best visualization of parathyroid adenoma on late images. (A) Left superior parathyroid adenoma is visualized on early images with tracer uptake approximately isointense with that of thyroid gland (from left to right: attenuation-corrected [AC] PET, PET/CT fusion, and maximum-intensity projection [MIP] images acquired at 5 min after injection). (B) On late images, adenoma is more apparent because of faster washout of tracer from thyroid gland (from left to right: AC PET, PET/CT fusion, and MIP images at 60 min after injection). 
The results of 2 scans were considered equivocal on both early and late images; 1 of these displayed an intrathyroidal hot spot with the differential diagnosis of thyroid adenoma, whereas the other showed an isointense lesion close to the thyroid. Histopathology proved that both lesions were parathyroid adenomas.

The scan in which the parathyroid gland was visualized only on late images was excluded from further quantitative analysis. For the cohort in total, the early SUV max $_{\text {m }}$ was significantly higher whereas the early SUV ${ }_{\max } \mathrm{P} / \mathrm{T}$ ratios were significantly lower than those on late images ( $P$ values were 0.001 for the absolute difference and 0.037 for the ratio difference; paired-samples $t$ test). More or less the same results were found for the measured $\operatorname{SUV}_{\text {peak }}(P$ values were $<0.001$ for the absolute difference and 0.021 for the ratio difference; paired-samples $t$ test) (Fig. 3).

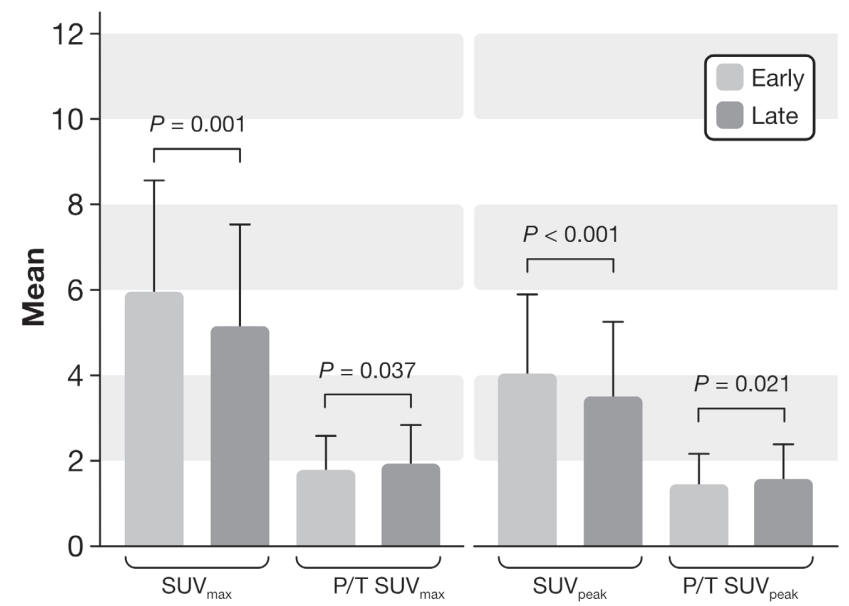

Figure 3. Comparison of early and late SUV measurements and P/T ratios.

Figure 4 shows several differences in quantitative results between the subgroups on the basis of visual interpretation. There was a decrease in ${ }^{18} \mathrm{~F}-\mathrm{FCH}$ uptake on late images for the subgroup with better visualization on early images; this result was more apparent for the absolute difference than for the ratio difference. There was a slight trend toward higher absolute ${ }^{18} \mathrm{~F}-\mathrm{FCH}$ uptake in the parathyroid lesions and higher relative uptake compared with that in the thyroid gland for the subgroup with better visualization on late images. Statistically, a significant difference was found between the subgroup with better visualization on early images and the subgroup with better visualization on late images ( $P$ values were 0.001 for the absolute difference and 0.001 for the ratio difference; Mann-Whitney $U$ test) as well as the subgroup with equivocal visualization ( $P$ values were $<0.001$ for the absolute difference and $<0.001$ for the ratio 
difference; Mann-Whitney $U$ test). No significant difference was found between the subgroup with better visualization on late images and the subgroup with equivocal visualization ( $P$ values were 0.221 for the absolute difference and 0.200 for the ratio difference; Mann-Whitney $U$ test). Analysis of the $S U V_{\text {peak }}$ measurements did not lead to different conclusions (Fig. 5).
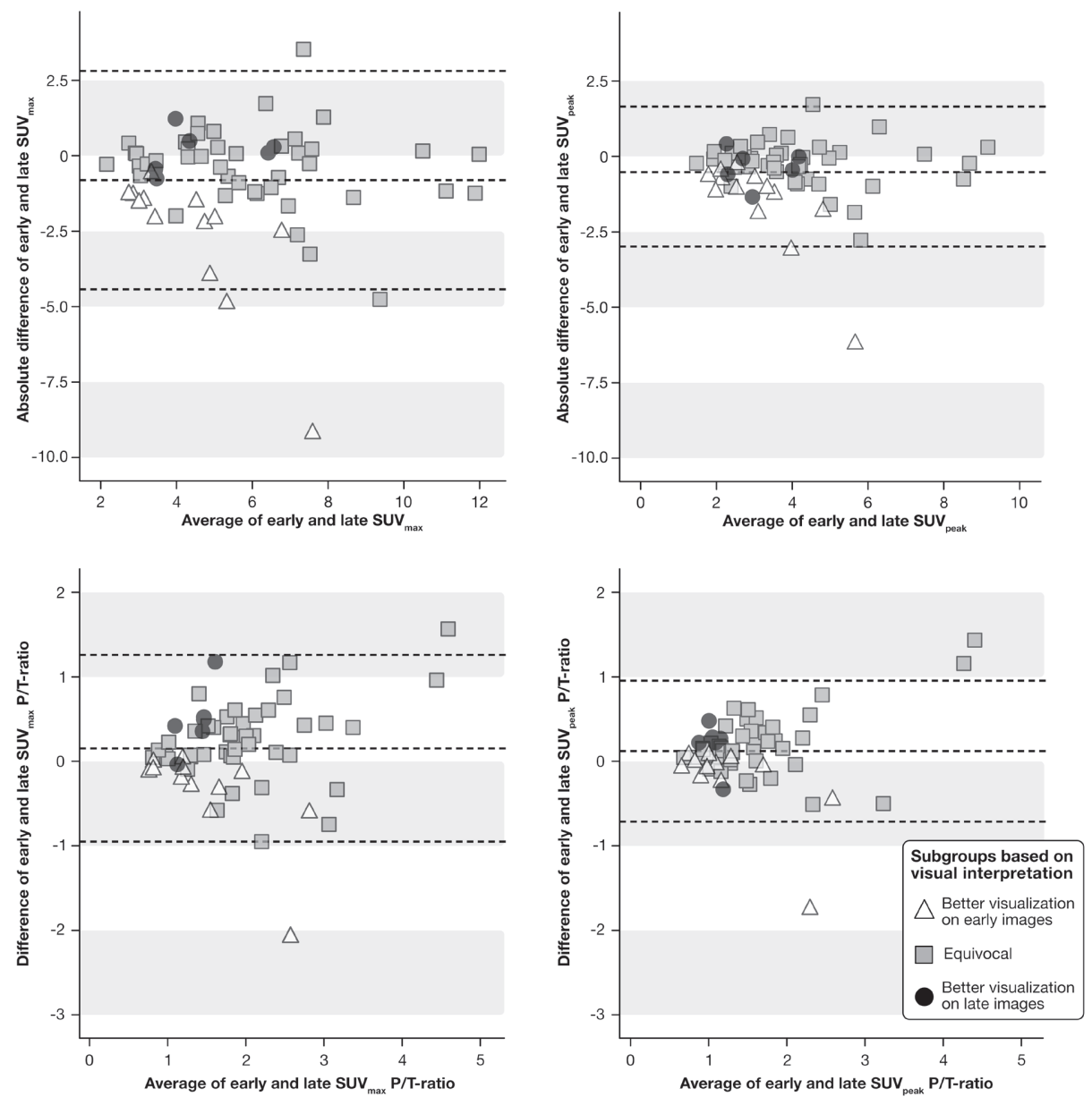

Figure 4. Bland-Altman plots. Plots of differences in ${ }^{18} \mathrm{~F}$-fluorocholine uptake $\left(\mathrm{SUV}_{\max }\right)$ in parathyroid lesions against mean uptake in each lesion (top left). Plots of differences in P/T ratios (SUV $\mathrm{V}_{\max }$ ) against mean ratio for each lesion (bottom left). For SUV peak' data are similarly plotted at top right and bottom right, respectively. Dotted lines are mean values and 95\% Cls for total cohort. 


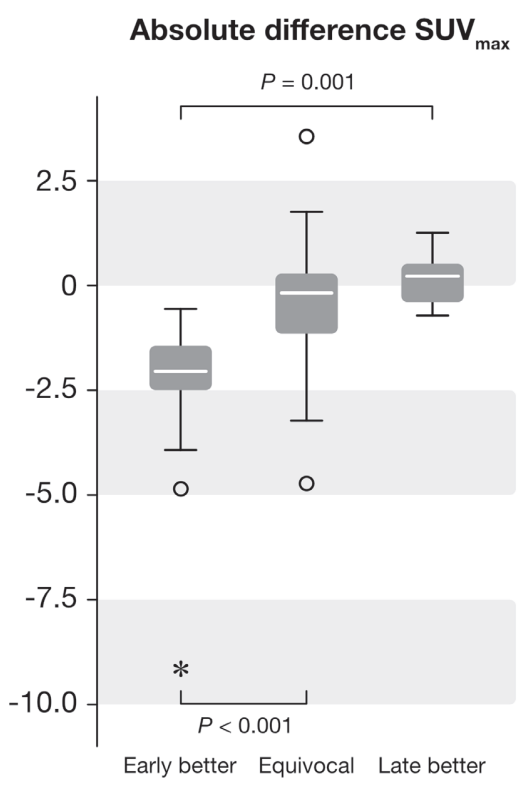

Absolute difference SUV peak
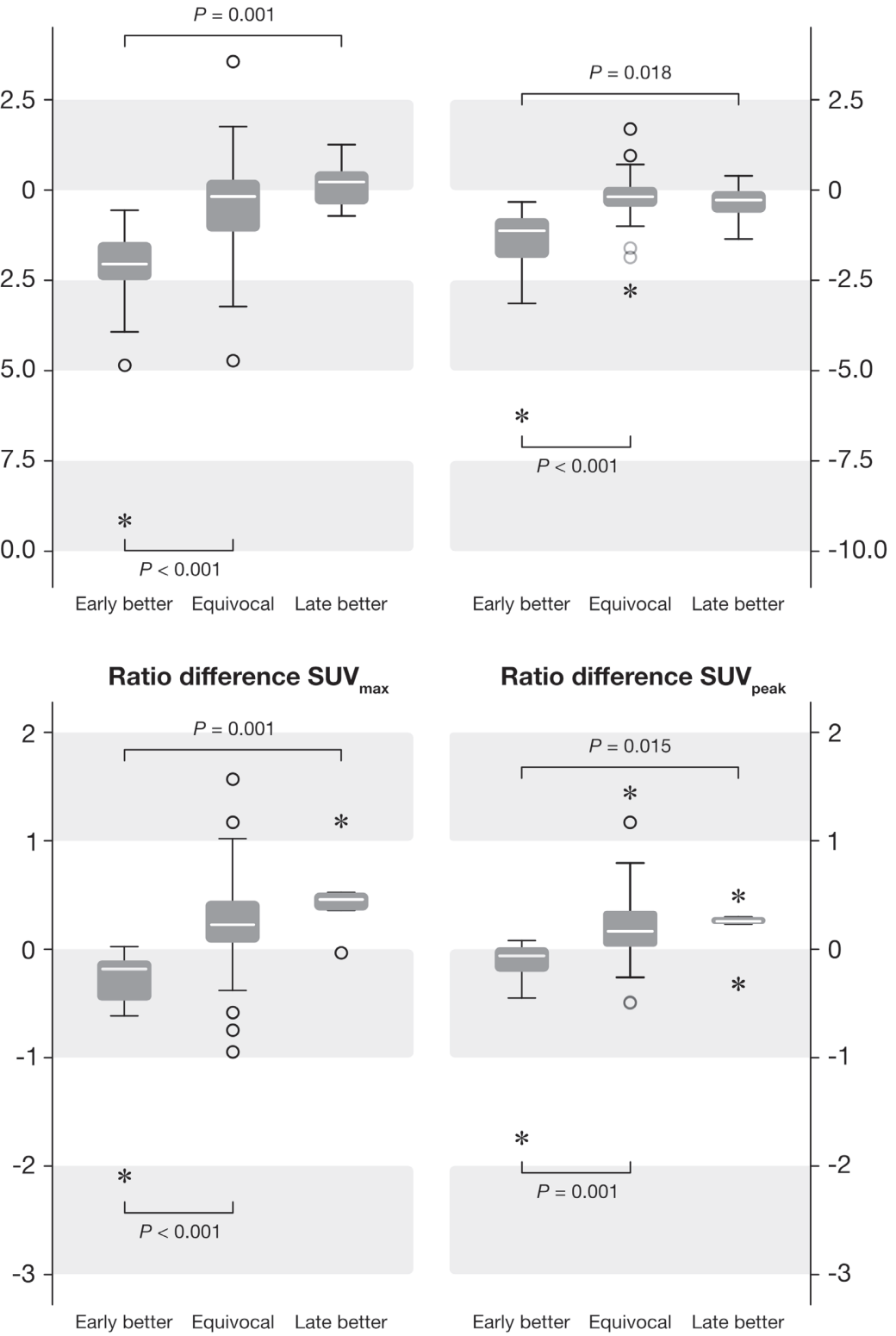

Figure 5. Differences between 3 subgroups on basis of visual interpretation: better visualization on early images (early better), equivocal visualization (equivocal), and better visualization on late images (late better). SUV max $_{\text {absolute differences (top left). SUV }}$ max ratio differences (bottom left). SUV peak $_{\text {absolute }}$

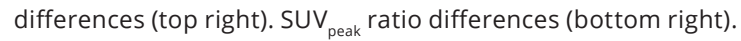




\section{Discussion}

In the present study, differences between parathyroid imaging with ${ }^{18} \mathrm{~F}-\mathrm{FCH}$ PET/CT at 5 min after injection and 60 min after injection were analyzed. Overall, parathyroid lesions showed higher ${ }^{18} \mathrm{~F}-\mathrm{FCH}$ uptake on early images and higher $\mathrm{P} / \mathrm{T}$ ratios on late images. However, different patterns were recognized, and scans with better visualization on early images as well as scans with better visualization on late images were observed.

In previous studies, multiple acquisition protocols were used in parathyroid imaging with ${ }^{18} \mathrm{~F}-\mathrm{FCH} \mathrm{PET} / \mathrm{CT}$, and several studies used a dual-time-point or triple-time-point protocol.[5,6,10-15] Lezaic et al. observed better lesion-to-background and lesion-tothyroid contrast on late images, but all lesions were visible at both time points.[10] Also, in 2 studies by Michaud et al., the late images did not yield additional findings. $[11,12]$ Two studies found an increase in the SUV ${ }_{\max }$ on late images, in contrast to the observations in the present study, and the results of 1 of those studies also suggested that a single late acquisition may suffice in patients with high PTH levels.[4,14] Some reports mentioned a dual-time-point scanning protocol without further explication of different scan results. $[5,13,15]$ In a study by Rep et al., the only study with a triple-timepoint protocol, the optimal scan time was concluded to be 60 min after injection of the tracer, with higher accuracy than early images and the same accuracy as images obtained at 120 min (accuracies of $94.1 \%, 96.5 \%$, and $96.5 \%$ at 5, 60, and 120 min after injection, respectively).[6] These results were due to nonvisualization of 3 parathyroid lesions on early images. Over time, a decrease in absolute uptake was observed, along with an increase in contrast relative to the thyroid gland, because of lower ${ }^{18} \mathrm{~F}-\mathrm{FCH}$ retention in the thyroid tissue - in agreement with the findings of the present study.

In the present study, none of the parathyroid lesions were exclusively visualized on early images, but failure to detect parathyroid lesions on delayed imaging has been reported in the literature. One case involved a cystic parathyroid adenoma with possible fast tracer washout due to a rich blood supply.[7] Because the highest activity was found in the first minutes on dynamic scans, another group of authors also hypothesized that a major portion of the SUV in the early phase represented blood flow in the adenoma.[16] However, no correlation between parathyroid vascularization and choline metabolism was found in a study comparing ${ }^{18} \mathrm{~F}-\mathrm{FCH}$ PET/CT at $10 \mathrm{~min}$ after injection with dual-phase contrast-enhanced CT (arterial phase images acquired 10-15 s after enhancement of the thoracic arch).[17] 
In the study of Rep et al., 1 adenoma could not be visualized on late images because of intense ${ }^{18} \mathrm{~F}-\mathrm{FCH}$ accumulation in bone marrow in 1 patient with polycythemia vera.[6] Fast choline washout was also observed in a small series of 7 patients who underwent a 30-min dynamic scan in combination with a static acquisition 60 min after injection. [18] Three different uptake patterns were recognized (early washout, stable uptake and late increase), indicating the importance of early acquisition for avoiding nondetection of parathyroid lesions with fast washout. Prabhu et al. prospectively compared early dynamic imaging during the first 15 min after injection with delayed imaging after 45 min. [8] A higher SUV $V_{\max }$ was observed on dynamic images, there was no significant difference in $\mathrm{P} / \mathrm{T}$ ratios between early dynamic and late images, and it was suggested that only early imaging would suffice.

For the analysis of ${ }^{18} \mathrm{~F}-\mathrm{FCH}$ uptake in the present study, both of the commonly used parameters (SUV ${ }_{\text {max }}$ and SUV ${ }_{\text {peak }}$ ) that were measured are known to have low interobserver variability (19). We decided not to use SUV ${ }_{\text {mean }}$ because it is strongly dependent on the way in which the volume of interest is drawn and therefore has higher interobserver variability, especially with small parathyroid lesions. In the present study, the use of SUV $_{\text {peak }}$ did not lead to different results than the use of SUV ${ }_{\text {max' }}$ and it seems that both methods are suitable for SUV measurements of parathyroid lesions.

Because the aim of the study was to analyze ${ }^{18} \mathrm{~F}-\mathrm{FCH}$ uptake patterns in hyperfunctioning parathyroid glands, we decided to include only histologically proven pathologic lesions. As a consequence, no difference in the accuracy of early versus late imaging could be calculated. Histopathologic examination was used as a reference standard in the present study. The histopathologic examination of parathyroid tissue can be difficult in certain cases; in particular, the distinction between adenoma and hyperplasia is not always clear. [20] Because of the small number of hyperplastic parathyroid glands versus parathyroid adenomas in the present study, no subgroup analysis was performed.

Additionally, evaluation of the correlations between uptake patterns, laboratory values, and the size or weight of the parathyroid glands may be of interest for future research. A known pitfall of ${ }^{18} \mathrm{~F}-\mathrm{FCH}$ PET/CT is tracer uptake in lymph nodes, which can cause false-positive results in parathyroid scanning, especially for foci in ectopic locations. Multiple-time-point scanning can help to identify benign lymph nodes.[21] Moreover, Prabhu et al. reported that early dynamic imaging was also able to differentiate between adenomas and cervical lymph nodes.[8] 
In the present study, patients did not have to discontinue calcimimetic drugs or other medications, except for colchicine, before PET/CT.[22] In previous studies on parathyroid imaging with ${ }^{18} \mathrm{~F}-\mathrm{FCH}$ PET/CT, discontinuation of parathyroid drugs also was not applied, but no specific research has been done on this matter. Moreover, with conventional ${ }^{99 m} \mathrm{Tc}$ sestamibi parathyroid imaging, the discontinuation of medications is also disputable. $[23,24]$

\section{Conclusion}

Variable ${ }^{18} \mathrm{~F}$-FCH uptake patterns were observed on dual-time-point ${ }^{18} \mathrm{~F}-\mathrm{FCH}$ PET/CT parathyroid imaging. Although most hyperfunctioning parathyroid glands were visualized on both early and late images, some lesions might have been missed if only 1 time point had been used. Therefore, we recommend the acquisition of dual-time-point images in parathyroid imaging with ${ }^{18} \mathrm{~F}-\mathrm{FCH}$ PET/CT or the creation of an opportunity to acquire additional late images after review of early images when findings are inconclusive. 


\section{References}

1. Adami S, Marcocci C, Gatti D. Epidemiology of primary hyperparathyroidism in europe.J Bone Miner Res 2002;17(suppl 2):N18-N23.

2. Lundgren E, Rastad J, Thrufjell E, Akerstrom G, Ljunghall S. Population-based screening for primary hyperparathyroidism with serum calcium and parathyroid hormone values in menopausal women. Surgery 1997;121:287-294.

3. Karipineni F, Sahli Z, Somervell H, et al. Are preoperative sestamibi scans useful for identifying ectopic parathyroid glands in patients with expected multigland parathyroid disease? Surgery 2018;163:35-41.

4. Thanseer N, Bhadada SK, Sood A, et al. Comparative effectiveness of ultrasonography, ${ }^{99 m T C-}$ sestamibi, and ${ }^{18} \mathrm{~F}$-fluorocholine PET/CT in detecting parathyroid adenomas in patients with primary hyperparathyroidism. Clin Nucl Med 2017;42:e491-e497.

5. Beheshti M, Hehenwarter L, Paymani Z, et al. ${ }^{18} \mathrm{~F}$-fluorocholine PET/CT in the assessment of primary

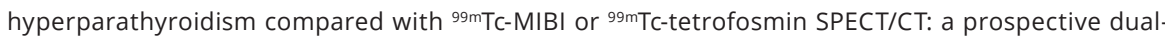
centre study in 100 patients. Eur J Nucl Med Mol Imaging 2018;45:1762-1771.

6. Rep S, Lezaic L, Kocjan T, et al. Optimal scan time for evaluation of parathyroid adenoma with ${ }^{18} \mathrm{~F}$-fluorocholine PET/CT. Radiol Oncol 2015;49:327-333.

7. Vellani C, Hodolic M, Chytiris S, Trifiro G, Rubello D, Colletti PM. Early and delayed ${ }^{18}$ F-FCH PET/CT imaging in parathyroid adenomas. Clin Nucl Med 2017;42:143-144.

8. Prabhu M, Kumari G, Damle NA, et al. Assessment of the role of early dynamic PET/CT with ${ }^{18} \mathrm{~F}$-fluorocholine in detection of parathyroid lesions in patients with primary hyperparathyroidism. Nucl Med Commun 2018;39:1190-1196.

9. Wilhelm SM, Wang TS, Ruan DT, et al. The American Association of Endocrine Surgeons guidelines for definitive management of primary hyperparathyroidism. JAMA Surg 2016;151:959-968.

10. Lezaic L, Rep S, Sever MJ, Kocjan T, Hocevar M, Fettich J. ${ }^{18}$ F-fluorocholine PET/CT for localization of hyperfunctioning parathyroid tissue in primary hyperparathyroidism: a pilot study. Eur J Nucl Med Mol Imaging 2014;41:2083-2089.

11. Michaud L, Burgess A, Huchet V, et al. Is ${ }^{18} \mathrm{~F}$-fluorocholine-positron emission tomography/computerized tomography a new imaging tool for detecting hyperfunctioning parathyroid glands in primary or secondary hyperparathyroidism? J Clin Endocrinol Metab 2014;99:4531-4536.

12. Michaud L, Balogova S, Burgess A, et al. A pilot comparison of ${ }^{18} \mathrm{~F}$-fluorocholine PET/CT, ultrasonography and ${ }^{123 /} /{ }^{99 m}$ Tc-sestaMIBI dual-phase dual-isotope scintigraphy in the preoperative localization of hyperfunctioning parathyroid glands in primary or secondary hyperparathyroidism: influence of thyroid anomalies. Medicine (Baltimore) 2015;94:e1701.

13. Hocevar M, Lezaic L, Rep S, et al. Focused parathyroidectomy without intraoperative parathormone testing is safe after pre-operative localization with ${ }^{18} \mathrm{~F}$-fluorocholine PET/CT. Eur J Surg Oncol 2017;43:133-137.

14. Iharbi AA, Alshehri FM, Albatly AA, et al. ${ }^{18} \mathrm{~F}$-fluorocholine uptake of parathyroid adenoma is correlated with parathyroid hormone level. Mol Imaging Biol 2018;20:857-867.

15. Bossert I, Chytiris S, Hodolic M, et al. PETC/CT with ${ }^{18} \mathrm{~F}$-choline localizes hyperfunctioning parathyroid adenomas equally well in normocalcemic hyperparathyroidism as in overt hyperparathyroidism. $J$ Endocrinol Invest 2019;42:419-426.

16. Prabhu M, Kumari G, Damle NA, et al. Comparability of early dynamic and conventional static imaging with ${ }^{18} \mathrm{~F}$-fluorocholine PET/CT in a patient with primary hyperparathyroidism. Clin Nucl Med 2018;43:e400-e401. 
17. Piccardo A, Trimboli P, Rutigliani $\mathrm{M}$, et al. Additional value of integrated ${ }^{18} \mathrm{~F}$-choline PET/4D contrastenhanced CT in the localization of hyperfunctioning parathyroid glands and correlation with molecular profile. Eur J Nucl Med Mol Imaging 2019;46:766-775.

18. Morland D, Richard C, Godard F, Deguelte S, Delemer B. Temporal uptake patterns of ${ }^{18}$ F-fluorocholine among hyperfunctioning parathyroid glands. Clin Nucl Med 2018;43:504-505.

19. Huang YE, Chen CF, Huang YJ, Konda SD, Appelbaum DE, Pu Y. Interobserver variability among measurements of the maximum and mean standardized uptake values on ${ }^{18} \mathrm{~F}-\mathrm{FDG}$ PET/CT and measurements of tumor size on diagnostic CT in patients with pulmonary tumors. Acta Radiol 2010;51:782-788.

20. Carlson D. Parathyroid pathology: hyperparathyroidism and parathyroid tumors. Arch Pathol Lab Med 2010;134:1639-1644.

21. Oprea-Lager DE, Vincent AD, van Moorselaar RJ, et al. Dual-phase PET-CT to differentiate ${ }^{18} \mathrm{~F}$-fluoromethylcholine uptake in reactive and malignant lymph nodes in patients with prostate cancer. PLoS One 2012;7:e48430.

22. Roef MJ, van der Poel H, van der Laken CJ, Vogel WV. Colchicine must be stopped before imaging with ${ }^{18}$ F-methylcholine PET/CT. Nucl Med Commun 2010;31:1075-1077.

24. Hindié E, Ugur O, Fuster D, et al. 2009 EANM parathyroid guidelines. Eur J Nucl Med Mol Imaging 2009;36:1201-1216.

25. Greenspan BS, Dillehay G, Intenzo C, et al. SNM practice guideline for parathyroid scintigraphy 4.0.J Nucl Med Technol 2012;40:111-118. 


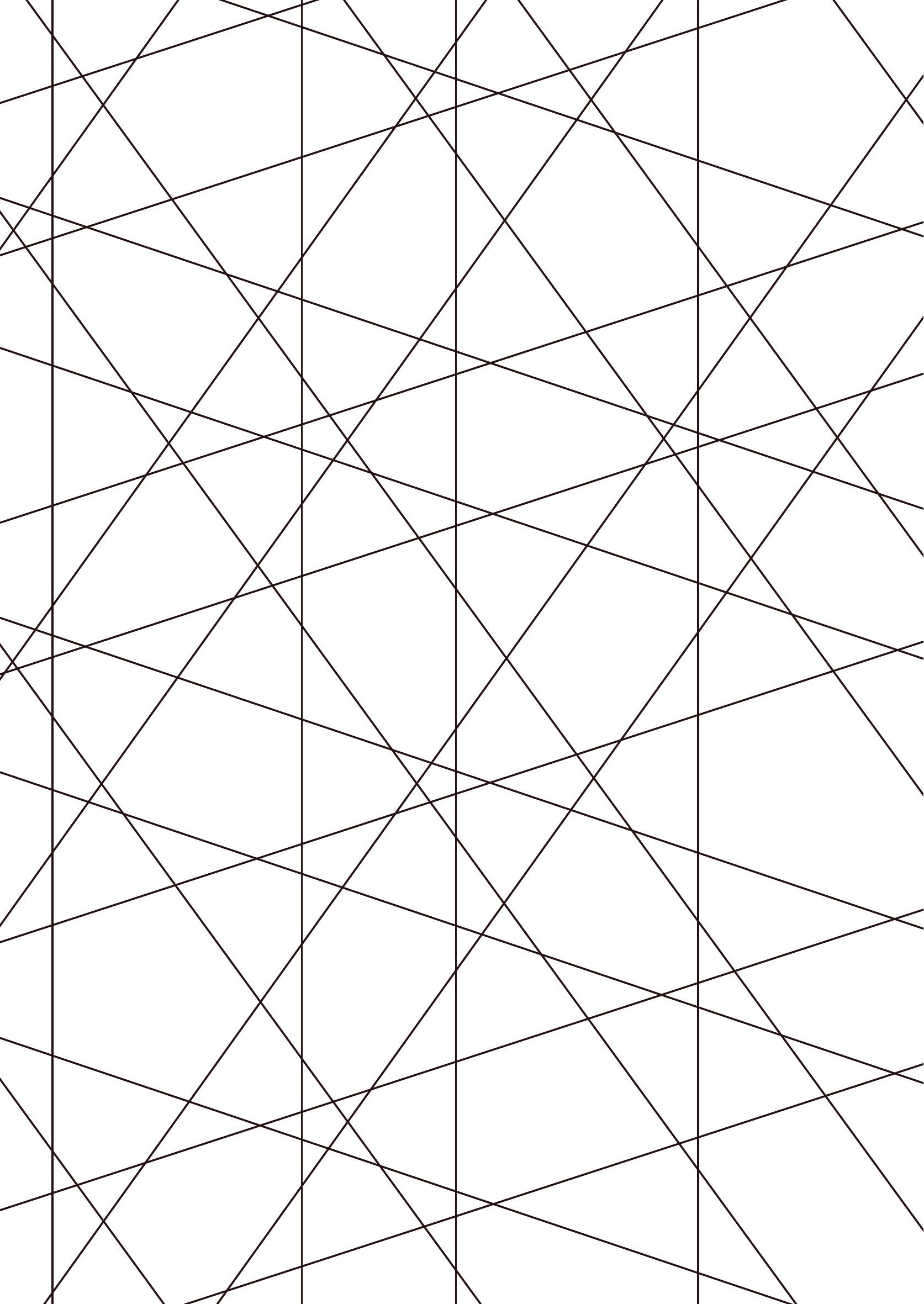




\title{
CHAPTER 4
}

Dynamic ${ }^{18} \mathrm{~F}$-fluorocholine PET/CT for parathyroid imaging in patients with primary hyperparathyroidism

\author{
Wouter A.M. Broos \\ Maurits Wondergem \\ Friso M. van der Zant \\ Nicolaas C. Schaper \\ Remco J.J. Knol \\ Nuclear Medicine Communications \\ 2020;41(8):776-782
}




\section{Abstract}

Background: In the past few years, ${ }^{18} \mathrm{~F}$-fluorocholine $\left({ }^{18} \mathrm{~F}-\mathrm{FCH}\right)$ PET/CT has been established as a promising imaging technique for preoperative localization of parathyroid adenomas, but the optimal time point to start PET/CT acquisition after tracer injection is yet unknown. The aim of the present study was to assess the optimal time frame to acquire the PET/ $\mathrm{CT}$ images and to evaluate the ability of dynamic imaging to differentiate parathyroid adenomas from active lymph nodes, a common cause for false-positive scan results.

Methods: Patients with primary hyperparathyroidism who had undergone a dynamic ${ }^{18} \mathrm{~F}-\mathrm{FCH}$ PET/CT positive for parathyroid disease and who subsequently underwent successful parathyroidectomy were retrospectively included in this study. On the 20 minutes dynamic images, standardized uptake value measurements were acquired per 1 minute frame for the parathyroid adenoma, the thyroid gland, blood pool activity, and, if present, lymph node activity.

Results: A total of 101 patients were included in this study. Time-activity curves showed a decrease of activity in parathyroid and thyroid glands, with faster wash-out from the thyroid gland and on average a stable, lower activity in lymph nodes. Blood pool activity was particularly present in the first 2 minutes. Differentiation of a parathyroid adenoma from active lymph nodes was best before 5 minutes, but no definitive cutoff value could be determined. Differentiation of a parathyroid adenoma from the thyroid gland was best after 10 minutes.

Conclusion: Dynamic imaging starting at the early time point of 2 minutes after injection of ${ }^{18} \mathrm{~F}-\mathrm{FCH}$ is useful for characterization of hyperfunctioning parathyroid glands. 


\section{Introduction}

Accurate preoperative localization of parathyroid disease is a prerequisite for minimally invasive parathyroidectomy.[1] Although conventional imaging techniques such as

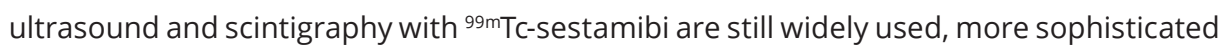
radiological imaging techniques, in particular MRI and multiphase contrast-enhanced CT (4D-CT), but also refined imaging techniques in nuclear medicine are gradually replacing conventional methods. Since the observation of high uptake of the tumor PET-tracer choline in a parathyroid adenoma in 2012 [2], several studies on the value of PET/CT in hyperparathyroidism were conducted, demonstrating favorable detection rates exceeding $90 \% .[3,4]$

Compared with ${ }^{99 m} \mathrm{Tc}$-sestamibi parathyroid scintigraphy, PET offers higher spatial resolution, shorter scanning time and less radiation dose.[5] However, several aspects of the acquisition protocol of ${ }^{18} \mathrm{~F}$-fluorocholine $\left({ }^{18} \mathrm{~F}-\mathrm{FCH}\right) \mathrm{PET} / \mathrm{CT}$ in hyperparathyroidism are under discussion. Previous studies have described the advantages of early image acquisition [6,7], but the optimal time point for scanning after injection of the tracer is yet to be determined. The ability of PET to continuously acquire data during a certain time frame, so-called dynamic scanning, enables reconstruction of tomographic images at multiple time points. This could help to assess the optimal time point for parathyroid imaging with PET/CT. Furthermore, it is known that on static choline PET/CT images, reactive lymph nodes can yield false-positive results.[8,9] This is due to nonspecific uptake of choline, which also accumulates in malignancies and reactive tissue. It has been suggested that dynamic scanning can differentiate lymph nodes from parathyroid glands.[9]

The objectives of the present study were to determine the optimal time point to acquire the ${ }^{18} \mathrm{~F}-\mathrm{FCH}$ PET/CT images and to assess the ability of dynamic imaging to differentiate parathyroid adenomas from active lymph nodes. 


\section{Methods}

\section{Patient selection}

Patients with primary hyperparathyroidism, who received a dynamic ${ }^{18} \mathrm{~F}-\mathrm{FCH}$ PET/ CT positive for parathyroid disease, and who subsequently underwent successful parathyroidectomy, were retrospectively included in this study. In our center, the ${ }^{18} \mathrm{~F}-\mathrm{FCH}$ $\mathrm{PET} / \mathrm{CT}$ is usually performed as the first-line imaging modality for parathyroid adenoma localization. After clinical evaluation of each PET/CT, baseline patient characteristics and scan results were entered into a database. Additional follow-up data were retrieved retrospectively from the electronic patient records.

\section{Informed consent}

The study passed the local scientific board and approval of the local ethical committee for the present study was waived since the study does not fall within the scope of the Dutch Medical Research Involving Human Subjects Act (section 1.b WMO, 26 February 1998). Furthermore, as a standard procedure on our department, all included patients gave written consent for usage of their data for scientific purposes. No additional measurements or actions affecting the patient were performed.

\section{Scan acquisition}

PET/CT images were acquired on a Biograph-16 TruePoint PET/CT camera (Siemens Healthineers, Erlangen, Germany). The intravenously injected activity was approximately $150 \mathrm{MBq}{ }^{18} \mathrm{~F}-\mathrm{FCH}$, produced at the on-site cyclotron facility. Dynamic images were acquired during 20 minutes after tracer injection, at one bed position ranging from the temporomandibular joint to the upper mediastinum. PET images were combined with low-dose CT for attenuation correction using a tube current of $40 \mathrm{mAs}$ at $120 \mathrm{kV}$ with CARE Dose 4D (Siemens Healthineers) dose modulation. Dynamic images were reconstructed to 20 frames of 1 minute. Additionally, an 8-minute static image containing data obtained from the fifth to $13^{\text {th }}$ minute after injection was reconstructed for visual analysis. Image interpretation and quantitative measurements were done with dedicated software (Syngo.via; Siemens Healthineers). Patient preparation consisted of hydration with $1 \mathrm{~L}$ of water and, if applicable, withdrawal of colchicine for $48 \mathrm{~h}$ prior to ${ }^{18} \mathrm{~F}-\mathrm{FCH}$ administration. Discontinuation of calcimimetic drugs or other medications was not required. 


\section{Image analysis}

Scans positive for a parathyroid adenoma that was visualized during the first 20 minutes after tracer injection were reviewed. In patients with more than one removed parathyroid gland, the gland with the highest choline uptake was selected for quantitative analysis. Additionally, if present, the most choline-avid lymph node was selected, which had to have a characteristic aspect of a lymph node on the low-dose CT and had to be located at typical sites, but not nearby typical parathyroid gland sites such as the thyroid region, along the carotid artery or in the mediastinum. Subsequently, on the dynamic images, a volume of interest (VOI) isocontour with a $40 \%$ threshold was drawn over the parathyroid focus, the thyroid gland and, if applicable, the selected lymph node (Fig. 1). Furthermore, a fixed VOI without threshold was drawn in the aortic arch to measure blood pool activity (Fig. 2). All VOIs were drawn on the dynamic frame with the best visualization of the structures and then copied to the same location on the other frames. Standardized uptake values (SUVs) in the VOIs were acquired for each time frame. SUV peak' $^{\prime}$ representing the mean SUV of a $1 \mathrm{~mL}$ spherical volume with the highest uptake within the drawn VOI, was thought to be the most robust measurement for choline activity, as SUV $_{\text {max }}$ representing a single voxel with the highest uptake, is more sensitive to noise and SUV mean the mean uptake of all voxels within the VOI, is strongly dependent on the way in which the VOI is drawn.[10] Therefore, SUV ${ }_{\text {peak }}$ was used to analyze the optimal acquisition time point in this study.
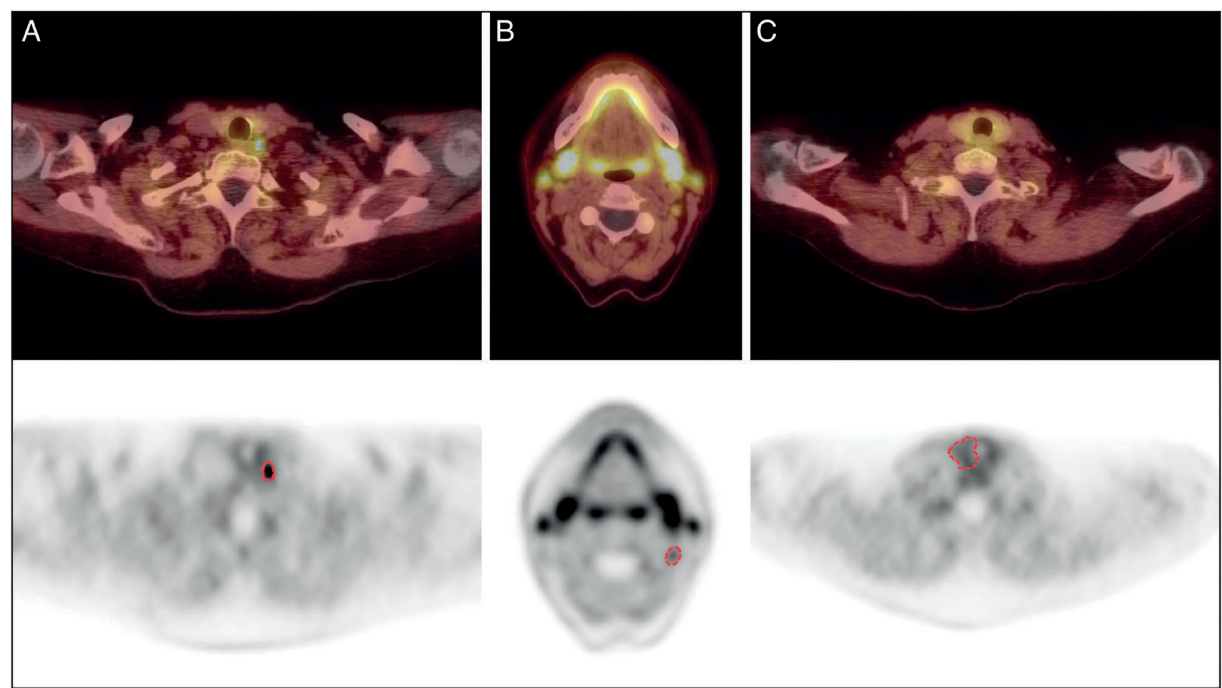

Figure 1. Examples of ${ }^{18} \mathrm{~F}$-fluorocholine PET/CT fusion images and the corresponding attenuation-corrected PET image below with volume of interest ( $40 \%$ threshold isocontour, red circle) of a left superior parathyroid adenoma (A), an active lymph node in left cervical level IIB (B), and thyroid gland (C). 


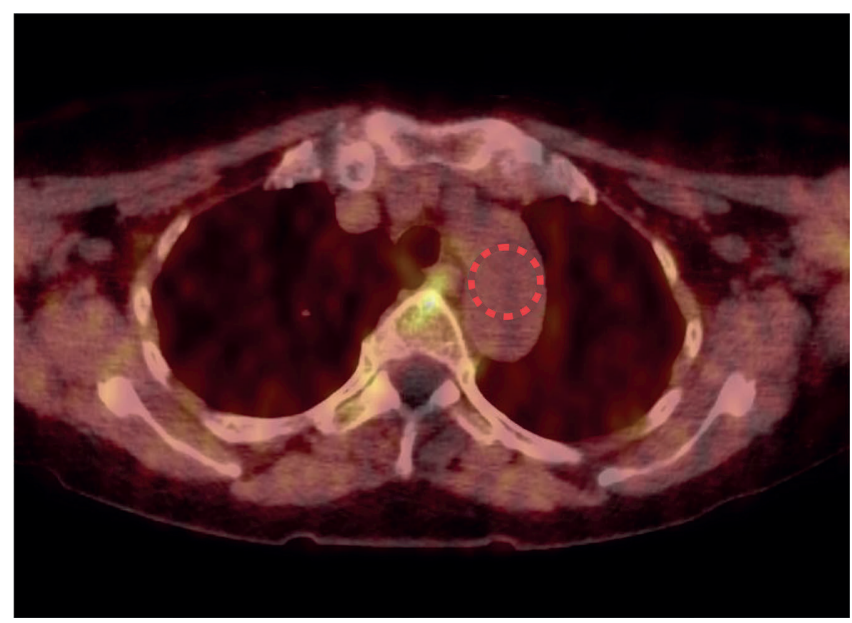

Figure 2. Example of a ${ }^{18} \mathrm{~F}$-fluorocholine PET/CT fusion image with a fixed volume of interest drawn over the aortic arch to measure blood pool activity (red circle).

\section{Statistical analysis}

Normally distributed continuous data were expressed as mean \pm SD and range. Noncontinuous data were expressed as numbers with percentages. Receiver operating characteristic (ROC) curves were drawn for parathyroid activity versus thyroid activity and for parathyroid activity versus lymph node activity. This was done for each time frame and areas under the curve were calculated to compare measurements. Statistical analysis was carried out using the Statistical Package for Social Sciences Version 25 (IBM SPSS Statistics).

\section{Results}

A total of 101 patients scanned from 31 March 2016 to 21 March 2019 were included in this study (Table 1). In 97 scans, a single hyperfunctioning parathyroid gland was visualized. Three scans showed two foci. In one of those patients only one parathyroid adenoma was found during surgery and in the other two patients both parathyroid adenomas were found at the corresponding locations. In one patient, three foci were visualized, of which only two were found during surgery. A total of 104 parathyroid glands were surgically removed of which 39 glands (37\%) were located left inferior to the thyroid gland, 43 glands (41\%) right inferior, nine glands (9\%) left superior, nine glands (9\%) right superior, and four glands were ectopically located in the mediastinum. All patients showed a significant decrease of intraoperatively measured parathyroid hormone (PTH) (at least a 50\% reduction) and reestablishment of normal calcium homeostasis lasting a minimum of 6 months. The mean weight of the removed glands was $0.93 \mathrm{~g}$ [SD 1.57, $95 \%$ confidence interval $(\mathrm{Cl}) 0.61-1.25$ ] and the mean volume was $1.72 \mathrm{~cm}^{3}$ (SD 3.70 , 
95\% Cl 0.87-2.57). At histopathology, 98 of the removed parathyroid glands proved to contain typical adenomas. Three glands showed hyperplasia and another three glands were confirmed to be parathyroid tissue without further specification, which were also considered as hyperfunctioning parathyroid glands because of the normalization of serum calcium and PTH after surgery. Figure 3 shows an example of several time points in a dynamic scan with visualization of a parathyroid adenoma.

The activity of a hyperfunctioning parathyroid gland was measured on all 101 included scans. On four scans, thyroid tissue could not be analyzed due to a history of thyroidectomy or thyroid hypoplasia and on seven scans, the blood pool activity could not be adequately measured because the aortic arch was located outside the scanning area. A choline-avid lymph node could be distinguished on 45 scans.

Table 1. Patient characteristics

\begin{tabular}{lc}
\hline Age [mean \pm SD (range)] (years) & $61 \pm 11(29-81)$ \\
Sex [n (\%)] & $21(21)$ \\
Male & $80(79)$ \\
Female & $17.8 \pm 10.2(6.3-73.6)$ \\
PTH [mean \pm SD (range)] (pmol/L) & \\
$\quad$ (normal range: $1.3-6.8$ pmol/L) & $2.72 \pm 0.18(2.28-3.31)$ \\
$\quad$ (normal range: $2.10-2.50 \mathrm{mmol} / \mathrm{L})$ &
\end{tabular}

The means of the SUV measurements for each time frame were plotted against time.

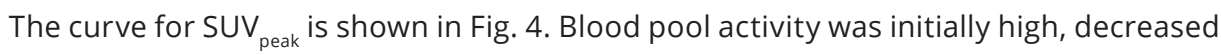
rapidly in the first 2-3 minutes, and was then followed by a slower decrease until the 20th minute. An overestimation of SUV measurements was noticeable in both parathyroid and thyroid glands in the first 2 minutes due to spillover of activity from nearby vascular structures. The parathyroid and thyroid gland demonstrated uptake which was typically slightly higher in the parathyroid glands, and activity in both tissues decreased gradually. The curves of the lymph nodes revealed a more steady activity during the 20 minutes scan. The mean activity of the lymph nodes exceeded the blood pool activity after 3-4 minutes, and remained lower than thyroid or parathyroid gland activity throughout the examination. However, regarding the spread of uptake values, there was an overlap between parathyroid adenoma and lymph node measurements, as is displayed for SUV $_{\text {peak }}$ in Fig. 5. A SUV-based cutoff point could therefore not be determined. 


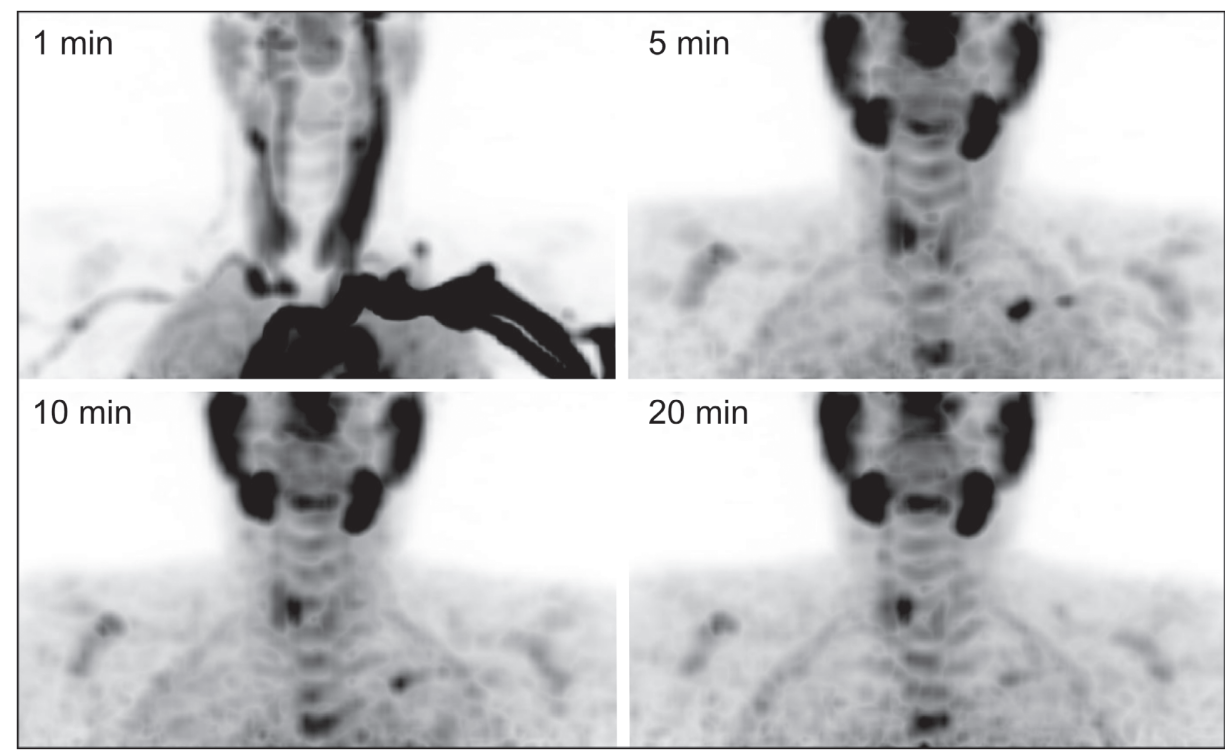

Figure 3. Dynamic ${ }^{18} \mathrm{~F}$-fluorocholine maximum-intensity projection images at four different time points, showing pathologic choline uptake in a parathyroid adenoma medial of the right thyroid lobe, physiologic uptake in salivary glands and bone marrow, rapidly decreasing blood pool activity and a slower wash-out of activity from the thyroid gland.

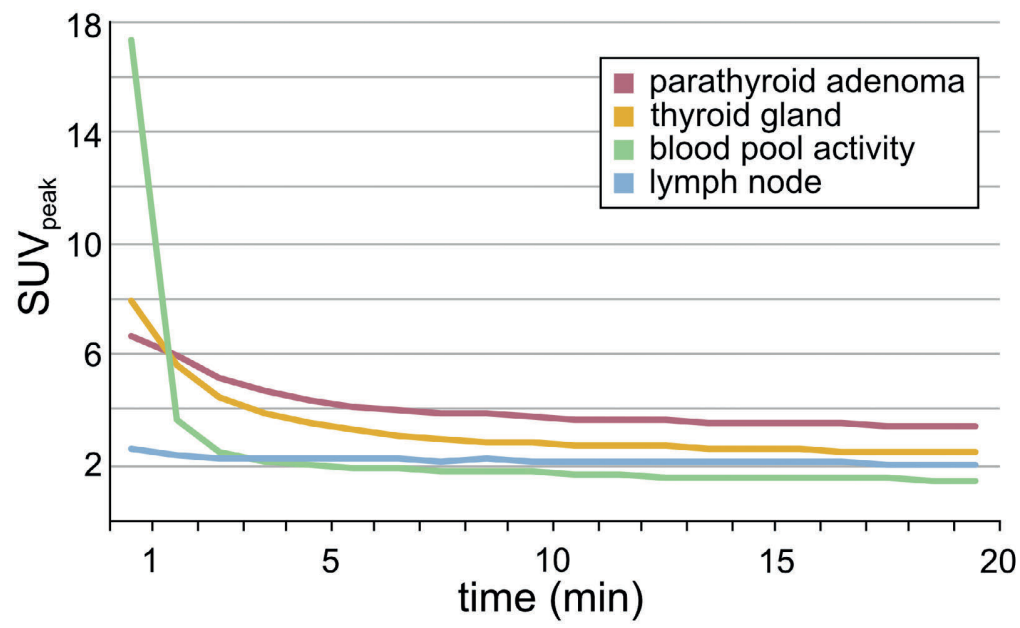

Figure 4. Time-activity curves of SUV $\mathrm{peak}_{\text {in }}$ four regions of interest plotted against 20 time frames of the dynamic ${ }^{18} \mathrm{~F}$-fluorocholine PET/CT scan. 


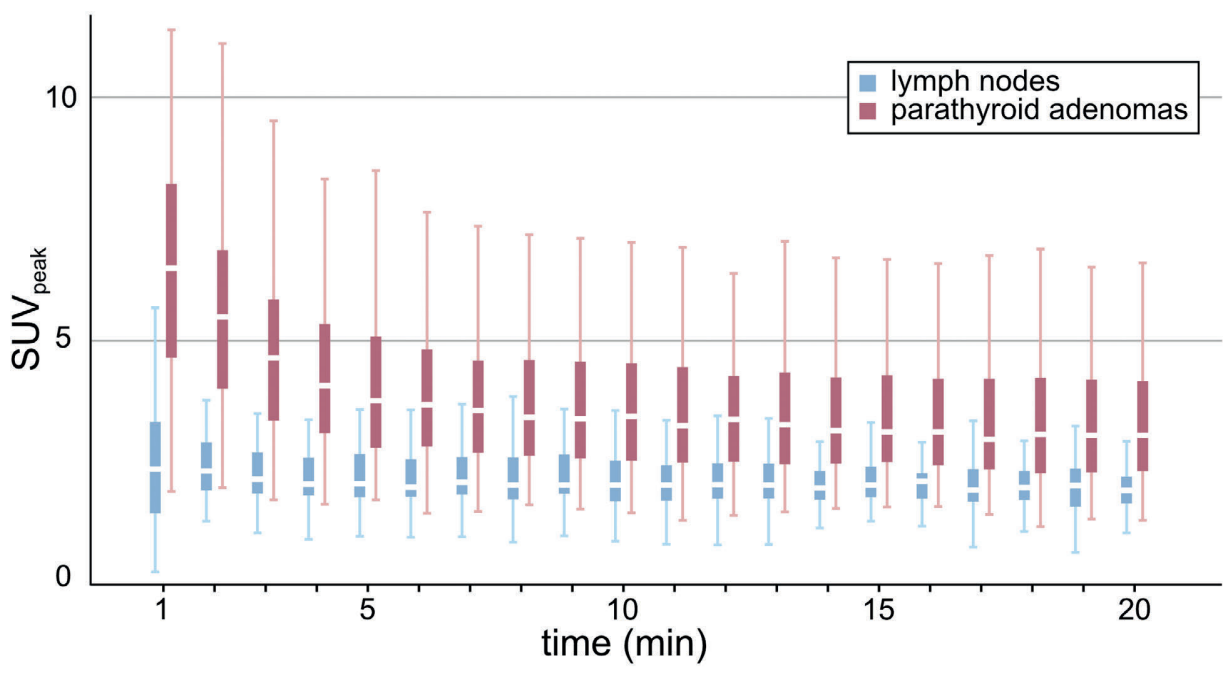

Figure 5. Boxplots showing the spread of uptake values $\left(S U V_{\text {peak }}\right)$ in measured lymph nodes (blue) and parathyroid glands (red). At each time point of the dynamic scan there is an overlap in activities between the two lesions.

To determine the optimal time point for SUV-based discrimination between thyroid gland and parathyroid adenoma, and also between active lymph nodes and parathyroid adenoma, ROC curves containing SUV peak measurements were drawn for each of the studied time points. Based on the corresponding areas under the curve, reflecting accuracy, the optimal time point for discrimination between the various tissues was found (Fig. 6). The highest accuracy based on ${ }^{18} \mathrm{~F}$-FCH uptake to differentiate parathyroid adenomas from thyroid tissue was found from 10 minutes onwards (accuracy of $~ 70 \%$ ). The highest accuracy for discrimination between parathyroid adenomas and lymph nodes was detected within the first 5 minutes of the PET/CT acquisition (accuracy of $>90 \%$ ). It must be emphasized that due to the aforementioned spillover of activity from the blood pool, the calculated accuracy in the first 2 minutes is unreliable. 


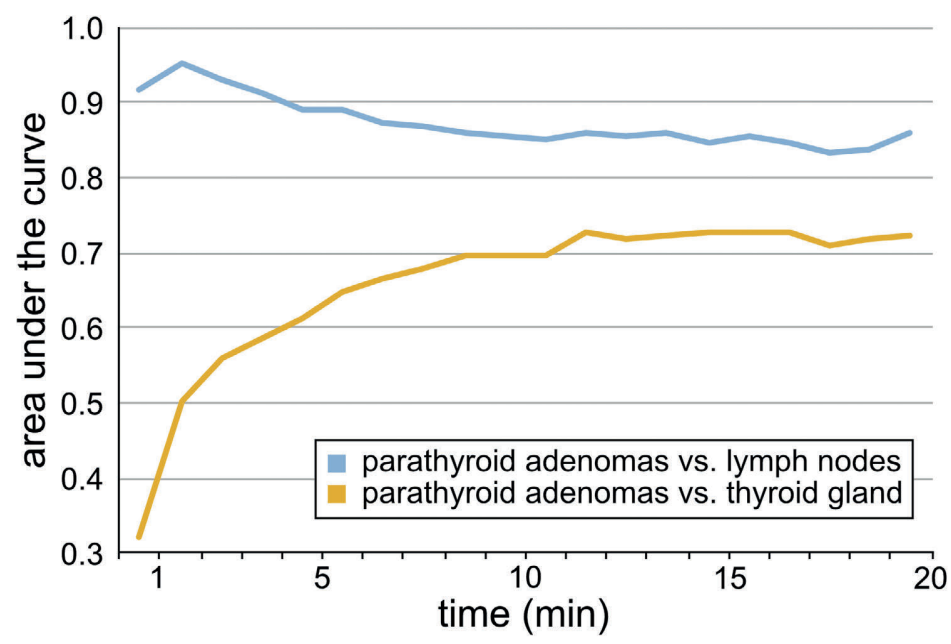

Figure 6. Area under the curve values plotted against time, derived from receiver operating characteristic curves between the activity of the parathyroid glands and lymph nodes (blue) and between the activity of the parathyroid glands and the thyroid glands (orange).

\section{Discussion}

Our data suggest that early dynamic scanning, starting 2 minutes after injection of the choline tracer, can help to characterize hyperfunctioning parathyroid glands. Choline serves as a precursor for phospholipid biosynthesis. Cells with a high proliferation rate have an increased demand for phospholipids, an essential component of cell membranes. After uptake of choline in the cell, the enzyme choline kinase phosphorylates the choline after which it is retained in the cell. Early high uptake of choline, observed in this study on the dynamic images, is probably partially resulting from high vascularity of adenomas, as was hypothesized previously by several authors.[11,12] This high vascularity is also visible on 4D-CT showing early contrast enhancement of parathyroid adenomas followed by wash-out.[13] However, due to retention of choline in the cells, uptake remains relatively high in these adenomas. In a study comparing choline PET/CT with 4D-CT, no correlation was found between choline uptake and vascularization of the parathyroid adenoma on arterial phase contrast enhanced CT (images acquired 10-15 seconds after enhancement of the thoracic arch).[14] However, no dynamic PET/CT images were available in that study, and since only static PET/CT images at 10 minutes after administration of the tracer were acquired, the role of vascularization on ${ }^{18} \mathrm{~F}-\mathrm{FCH}$ uptake in the first minutes could not be assessed. Another issue in reading early choline PET/CT images is the intense blood pool activity, in the present study especially in the first 2 minutes, which can obscure possible lesions. Furthermore, spillover of activity from adjacent vessels 
such as the jugular vein can cause overestimation of activity in both the parathyroid and thyroid gland. Due to this high blood pool activity, it seems preferable to delay scanning with at least 2 minutes after administration of the tracer.

Although numerous articles on choline PET/CT for preoperative localization of parathyroid adenomas were published in the last decade, only few studies, all with small cohort sizes, used dynamic scanning. The main strengths of the present study are the large cohort size and additionally histopathological confirmation of all lesions. In two studies by Michaud et al.[15,16], 10 minutes dynamic scans were acquired. The authors noticed that all the abnormal foci detected on the static PET acquisition, started 10 minutes after injection, were already visible on the early dynamic images, although vascular activity complicated the interpretation of these images. No further analysis of the dynamic images was reported in these studies. In a study by Prabhu et al.[9], 14 patients were dynamically scanned for 15 minutes directly after tracer administration, and in a case series by Morland et al.[17], seven patients received a dynamic PET/CT scan (approximately for 30 minutes, started 3 minutes after injection of the tracer). In both studies, high early choline uptake in adenomas followed by a gradual decrease was observed, which is in accordance with the observations in the present study. One previous study focused on the optimal scanning time with choline PET/CT [18]; however, ${ }^{11} \mathrm{C}$-choline instead of ${ }^{18} \mathrm{~F}-\mathrm{FCH}$ was used as the tracer. In that study, nine patients were dynamically scanned for up to 40-60 minutes, SUV measurements of the thyroid gland and parathyroid adenoma were performed and thyroid to parathyroid ratios were calculated on 10 minutes frames. It was concluded that the time frame of 20-30 minutes was preferable because the ratio did not essentially increase after this time frame. In the present study, the optimal contrast between thyroid and parathyroid was reached after approximately 10 minutes, with a moderate SUV-based accuracy of $70 \%$ (Fig. 6), which is at least 10 minutes earlier than in the aforementioned study. Possibly, this is due to slightly differing properties between the used tracers.[19] Besides, it should be pointed out that this is not the accuracy to detect the parathyroid adenoma, but to differentiate the parathyroid from the thyroid gland purely based on SUV.

We also investigated the ability of ${ }^{18} \mathrm{~F}-\mathrm{FCH}$ PET/CT to differentiate between the parathyroid adenomas and lymph nodes. Earlier studies of choline PET/CT in prostate cancer patients showed that choline not only accumulates in lymph node metastases, but also in reactive lymph nodes.[20] Uptake in such lymph nodes can complicate the interpretation of parathyroid scans, as lymph nodes can be present at locations similar to parathyroid adenomas. This is especially true for ectopic adenomas, which due to migratory descent from the pharyngeal pouches during embryogenesis, may be located anywhere from 
the level of the hyoid bone to the lower mediastinum.[21] The study by Prabhu et al.[9] revealed time-to-peak activity in lymph nodes after 6 minutes compared to the time-topeak activity in parathyroid adenomas after 2 minutes. In the present study, however, time-to-peak activity was approximately equal for all measured structures, and therefore unable to differentiate those structures. Discrimination between parathyroid adenomas and lymph nodes was optimal between 2 and 5 minutes after tracer injection (accuracy of $>90 \%$; Fig. 6). Activity in lymph nodes was often lower than in adenomas but a clear SUV cutoff value could not be assessed. Therefore, SUV measurements are of limited use to discriminate between parathyroid lesions and lymph nodes, and the uptake pattern that is observed on dynamic images seems more useful. No histopathological confirmation of the lymph nodes was acquired, but the lymph nodes were selected in regions of the neck in which presence of a parathyroid adenoma was highly unlikely. Due to the study design, no sensitivity or specificity in differentiating parathyroid adenomas from benign lymph nodes could be adequately calculated. Scanning at a later time point, usually 60 minutes after injection, could also help in differentiating lymph nodes, since benign lymph nodes are known to show wash-out of the tracer over time.[8] It has also been suggested that combining choline PET with 4D-CT may enable differentiation between adenomas and lymph nodes by grading of the arterial phase of contrast enhancement. [22] Additionally, combination of these two techniques may improve the accuracy of parathyroid adenoma localization.[14,23]

In the present study, the optimal time point to start PET/CT acquisition was determined, but not the optimal scan duration as was done in the study by Noltes et al.[18]. No further attempt was made to determine the optimal scan duration with the dynamic data available in the present study, as this is strongly related to several parameters that differ between clinical centers including administered activity, technical capabilities of the used PET scanner, and type of post processing of the acquired PET data, which hampers generalization of collected data and recommendations on this topic. Another point that should be noted is that dynamic imaging limits the scan range to one bed position, which is often not enough to cover the total area of possible adenoma locations, and therefore a static image of two or more bed positions is still required. Since the introduction of ${ }^{18} \mathrm{~F}-\mathrm{FCH}$ PET/CT in our hospital in 2015, patients with primary hyperparathyroidism have been receiving this imaging procedure as the first-line imaging method for localizing parathyroid lesions. In a few patients, previous imaging results were available, including

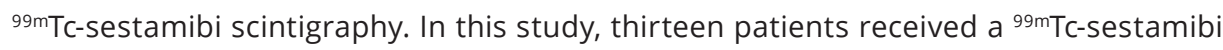
scan; seven with negative results and six with positive results which were concordant with ${ }^{18} \mathrm{~F}-\mathrm{FCH}$ PET/CT results. 
All in all, this study points out that since scanning in the first 2 minutes after injection of the tracer is useless due to spillover of blood pool activity from adjacent vascular structures, acquisition of ${ }^{18} \mathrm{~F}-\mathrm{FCH}$ PET/CT should be started at least 2 minutes after tracer injection. Because the parathyroid glands are commonly located nearby the thyroid gland, it is important to differentiate the activity in a parathyroid adenoma from the physiological uptake in the thyroid gland. The optimal contrast between parathyroid and thyroid tissue was observed from 10 minutes after ${ }^{18} \mathrm{~F}-\mathrm{FCH}$. Furthermore, it is necessary that a choline-avid lymph node is not interpreted as a parathyroid adenoma. The differentiation of lymph nodes from parathyroid adenomas based on SUV was best during the first 5 minutes of the acquisition, but no definitive cutoff value could be determined. Acquisition of dynamic images also enables reconstruction of time-activity curves that may be helpful to differentiate hyperfunctioning parathyroid tissue from choline-avid lymph nodes, since activity in parathyroid tissue often showed a rapidly decreasing activity in the early minutes of the scan, while a more stable activity over time was more indicative for a lymph node. Early dynamic image acquisition starting at 2 minutes after tracer injection is therefore the preferable protocol to choose for ${ }^{18} \mathrm{~F}-\mathrm{FCH}$ PET/CT imaging. When acquisition of dynamic images is not feasible, the optimal time point to acquire static images is 10 minutes after tracer administration, since from this time point parathyroid lesions are less prone to be obscured by thyroid activity which is particularly present earlier. However, benign active lymph nodes seem to remain a source for false-positive or false-negative results.

\section{Conclusion}

Dynamic imaging starting at the early time point of 2 minutes after injection of ${ }^{18} \mathrm{~F}-\mathrm{FCH}$ is useful for characterization of hyperfunctioning parathyroid glands. 


\section{References}

1. Sackett WR, Barraclough B, Reeve TS, Delbridge LW. Worldwide trends in the surgical treatment of primary hyperparathyroidism in the era of minimally invasive parathyroidectomy. Arch Surg 2002;137:1055-1059.

2. Mapelli P, Busnardo E, Magnani P, Freschi M, Picchio M, Gianolli L, Messa C. Incidental finding of parathyroid adenoma with ${ }^{11} \mathrm{C}$-choline PET/CT. Clin Nucl Med 2012;37:593-595.

3. Treglia G, Piccardo A, Imperiale A, Strobel K, Kaufmann PA, Prior JO, Giovanella L. Diagnostic performance of choline PET for detection of hyperfunctioning parathyroid glands in hyperparathyroidism: a systematic review and meta-analysis. Eur J Nucl Med Mol Imaging 2019;46:751765.

4. Broos WAM, van der Zant FM, Knol RJJ, Wondergem M. Choline PET/CT in parathyroid imaging: a systematic review. Nucl Med Commun 2019;40:96-105.

5. Rep S, Hocevar M, Vaupotic J, Zdesar U, Zaletel K, Lezaic L. ${ }^{18} \mathrm{~F}$-choline PET/CT for parathyroid scintigraphy: significantly lower radiation exposure of patients in comparison to conventional nuclear medicine imaging approaches. J Radiol Prot 2018;38:343-356.

6. Rep S, Lezaic L, Kocjan T, Pfeifer M, Sever MJ, Simoncic U, et al. Optimal scan time for evaluation of parathyroid adenoma with [18 $\mathrm{F}]-$ fluorocholine PET/CT. Radiol Oncol 2015;49:327-333.

7. Broos WAM, Wondergem M, van der Zant FM, Knol RJJ. Dual-time-point ${ }^{18} \mathrm{~F}$-fluorocholine PET/CT in parathyroid imaging. J Nucl Med 2019;60:1605-1610.

8. Oprea-Lager DE, Vincent AD, van Moorselaar RJ, Gerritsen WR, van den Eertwegh AJ, Eriksson J, et al. Dual-phase PET-CT to differentiate $\left[{ }^{18} \mathrm{~F}\right]$ fluoromethylcholine uptake in reactive and malignant lymph nodes in patients with prostate cancer. PLoS One 2012;7:e48430.

9. Prabhu M, Kumari G, Damle NA, Arora G, Kumar P, Kumar R, et al. Assessment of the role of early dynamic PET/CT with ${ }^{18} \mathrm{~F}$-fluorocholine in detection of parathyroid lesions in patients with primary hyperparathyroidism. Nucl Med Commun 2018;39:1190-1196.

10. Lodge MA, Chaudhry MA, Wahl RL. Noise considerations for PET quantification using maximum and peak standardized uptake value. J Nucl Med 2012; 53:1041-1047.

11. Vellani C, Hodolič M, Chytiris S, Trifirò G, Rubello D, Colletti PM. Early and delayed ${ }^{18}$ F-FCH PET/CT imaging in parathyroid adenomas. Clin Nucl Med 2017;42:143-144.

12. Prabhu M, Kumari G, Damle NA, Arora G, Tripathi M, Bal C, et al. Comparability of early dynamic and conventional static imaging with ${ }^{18} \mathrm{~F}$-fluorocholine $\mathrm{PET} / \mathrm{CT}$ in a Patient with primary hyperparathyroidism. Clin Nucl Med 2018;43:e400-e401.

13. Raghavan $P$, Durst CR, Ornan DA, Mukherjee S, Wintermark M, Patrie JT, et al. Dynamic CT for parathyroid disease: are multiple phases necessary? AJNR Am J Neuroradiol 2014;35:1959-1964.

14. Piccardo A, Trimboli P, Rutigliani M, Puntoni M, Foppiani L, Bacigalupo L, et al. Additional value of integrated ${ }^{18} \mathrm{~F}$-choline PET/4D contrast-enhanced CT in the localization of hyperfunctioning parathyroid glands and correlation with molecular profile. Eur J Nucl Med Mol Imaging 2019;46:766-775.

15. Michaud L, Burgess A, Huchet V, Lefèvre M, Tassart M, Ohnona J, et al. Is ${ }^{18} \mathrm{~F}$-fluorocholine-positron emission tomography/computerized tomography a new imaging tool for detecting hyperfunctioning parathyroid glands in primary or secondary hyperparathyroidism? J Clin Endocrinol Metab 2014;99:4531. 4536.

16. Michaud L, Balogova S, Burgess A, Ohnona J, Huchet V, Kerrou K, et al. A pilot comparison of ${ }^{18} \mathrm{~F}$-fluorocholine PET/CT, ultrasonography and ${ }^{123} \mathrm{I} / 99 \mathrm{~m} T \mathrm{C}$-sestaMIBI Dual-phase dual-isotope scintigraphy in the preoperative localization of hyperfunctioning parathyroid glands in primary or secondary hyperparathyroidism: influence of thyroid anomalies. Medicine (Baltimore) 2015;94:e1701. 
17. Morland D, Richard C, Godard F, Deguelte S, Delemer B. Temporal uptake patterns of ${ }^{18} \mathrm{~F}$-fluorocholine among hyperfunctioning parathyroid glands. Clin Nucl Med 2018;43:504-505.

18. Noltes ME, Kruijff S, Noordzij W, Telenga ED, Vállez García D, Trofimiuk- Müldner M, et al. Optimization of parathyroid ${ }^{11} \mathrm{C}$-choline PET protocol for localization of parathyroid adenomas in patients with primary hyperparathyroidism. EJNMMI Res 2019;9:73.

19. Haroon A, Zanoni L, Celli M, Zakavi R, Beheshti M, Langsteger W, et al. Multicenter study evaluating extraprostatic uptake of ${ }^{11} \mathrm{C}$-choline, ${ }^{18} \mathrm{~F}$-methylcholine, and ${ }^{18} \mathrm{~F}$-ethylcholine in male patients: physiological distribution, statistical differences, imaging pearls, and normal variants. Nucl Med Commun 2015;36:1065-1075.

20. Evangelista L, Zattoni F, Guttilla A, Saladini G, Zattoni F, Colletti PM, Rubello D. Choline PET or PET/ CT and biochemical relapse of prostate cancer: a systematic review and meta-analysis. Clin Nucl Med 2013;38:305-314.

21. Lappas D, Noussios G, Anagnostis P, Adamidou F, Chatzigeorgiou A, Skandalakis P. Location, number and morphology of parathyroid glands: results from a large anatomical series. Anat Sci Int 2012;87:160164.

22. Christakis I, Khan S, Sadler GP, Gleeson FV, Bradley KM, Mihai R. ${ }^{18}$ Fluorocholine PET/CT scanning with arterial phase-enhanced $C T$ is useful for persistent/recurrent primary hyperparathyroidism: first UK case series results. Ann R Coll Surg Eng/ 2019;101:501-507.

23. Amadou C, Bera G, Ezziane M, Chami L, Delbot T, Rouxel A, et al. ${ }^{18} \mathrm{~F}$-fluorocholine PET/CT and parathyroid 4D computed tomography for primary hyperparathyroidism: the challenge of reoperative patients. World J Surg 2019;43:1232-1242. 


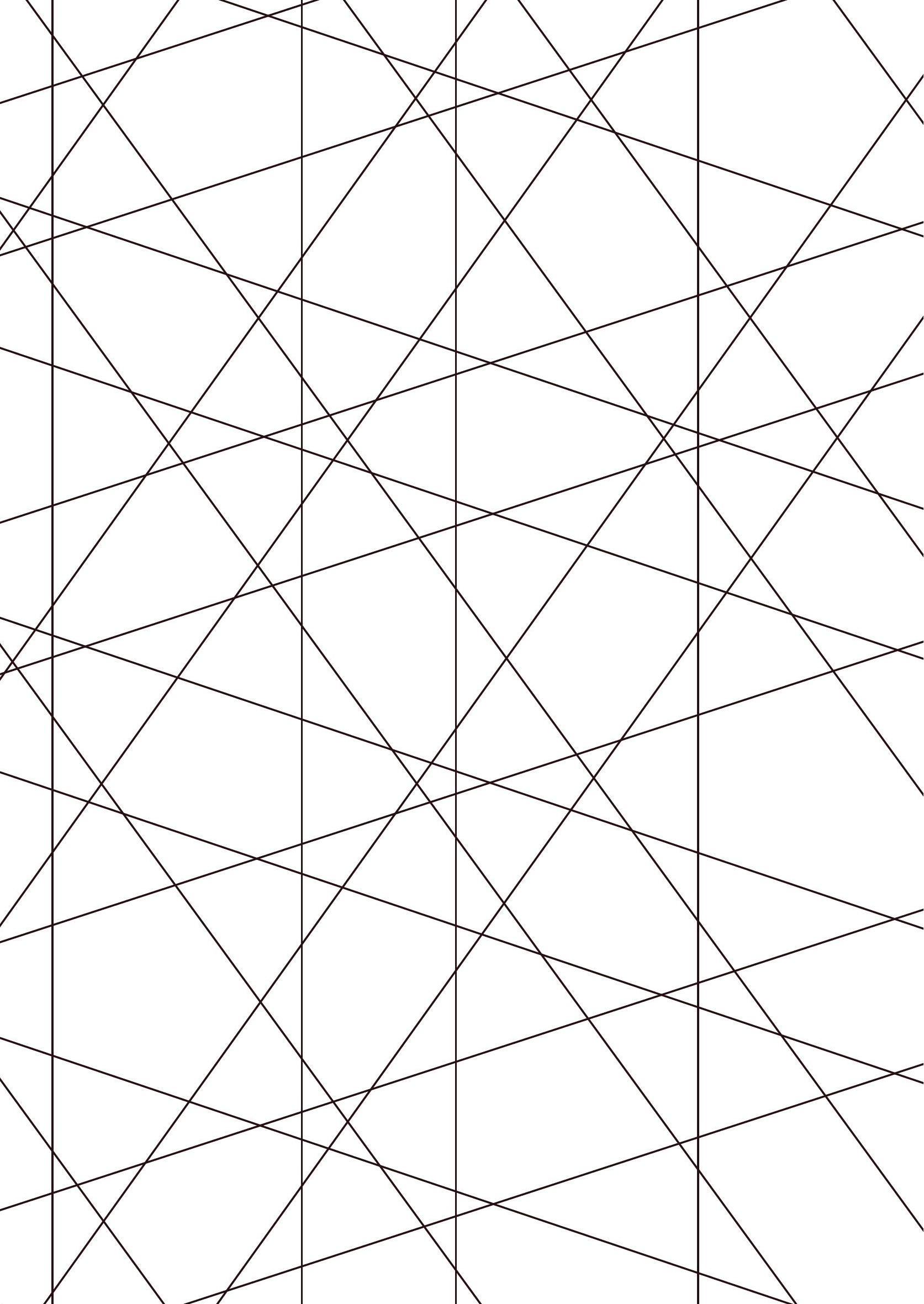




\section{INTERMEZZO}

Tertiary hyperparathyroidism with renal osteodystrophy on ${ }^{18} \mathrm{~F}$-fluorocholine PET/CT

Wouter A.M. Broos

Maurits Wondergem

Friso M. van der Zant

Remco J.J. Knol

Clinical Nuclear Medicine

2018;43(10):766-768 
A 27-year-old man, with a history of chronic renal failure due to obstructive uropathy, treated with hemodialysis and awaiting kidney transplantation, presented with tertiary hyperparathyroidism. Despite regulation of phosphate and calcium, and treatment with cinacalcet, the serum parathyroid hormone levels remained elevated. The patient was referred for parathyroidectomy, and a ${ }^{18} \mathrm{~F}$-fluorocholine $\left({ }^{18} \mathrm{~F}-\mathrm{FCH}\right) \mathrm{PET} / \mathrm{CT}$ was acquired for preoperative planning (Fig. 1). Besides visualization of the hyperfunctioning parathyroid glands, the scan also showed a typical picture of renal osteodystrophy (Fig. 2), a finding on ${ }^{18} \mathrm{~F}-\mathrm{FCH}$ PET/CT that has not been reported before.

Besides its wider use in imaging of malignancies such as prostate cancer, ${ }^{18} \mathrm{~F}-\mathrm{FCH}$ PET/ $\mathrm{CT}$ has recently proven very promising for imaging of hyperfunctioning parathyroid tissue.[1,2] In secondary and tertiary hyperparathyroidism, which can be treated with parathyroidectomy, imaging is mainly performed to detect ectopic or supernumerary glands.[3] In this case, all glands were eutopic and no supernumerary glands were detected. The patient underwent total parathyroidectomy with autotransplantation of approximately $2 \mathrm{~g}$ of fragmented parathyroid tissue into the right sternocleidomastoid muscle. Intraoperative serum parathyroid hormone testing showed a decrease from $341.7 \mathrm{pmol} / \mathrm{L}$ to $43.9 \mathrm{pmol} / \mathrm{L}$. Histopathologic examination of the excised glands revealed hyperplasia.

Renal osteodystrophy occurs in chronic kidney disease, due to a complex pathophysiology, which involves a number of feedback loops between kidney, bone, intestine, and vasculature (a systemic condition also known as chronic kidney disease-mineral and bone disorder). This results in a variety of bone abnormalities comprising osteosclerosis, osteomalacia, osteoporosis, pathologic fractures, osteonecrosis, and bone pain.[4,5] Historically, renal osteodystrophy has been imaged with conventional bone scintigraphy, using ${ }^{99 \mathrm{~m} T c-l a b e l e d ~ d i p h o s p h o n a t e s .[6] ~ T w o ~ m o r e ~ r e c e n t ~ p u b l i c a t i o n s ~ r e p o r t e d ~ o n ~ t h e ~ u s e ~}$ of ${ }^{18} \mathrm{~F}$-FDG PET/CT in this disease.[7,8] Moreover, visualization of renal osteodystrophy

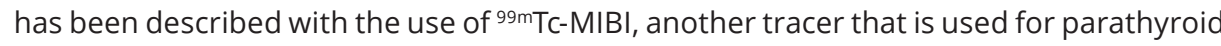
imaging.[9] However, to the best of our knowledge, renal osteodystrophy imaged by ${ }^{18} \mathrm{~F}-\mathrm{FCH}$ PET/CT has not been reported before. After surgery, the patient developed a hungry bone syndrome, requiring extensive calcium supplementation. Although this complication is rare, it has been reported to occur more frequently in patients with radiological evidence of hyperparathyroidism-related bone disease.[10] This, in addition, indicates the importance of recognizing bone disease on preoperative imaging. 


\section{A}

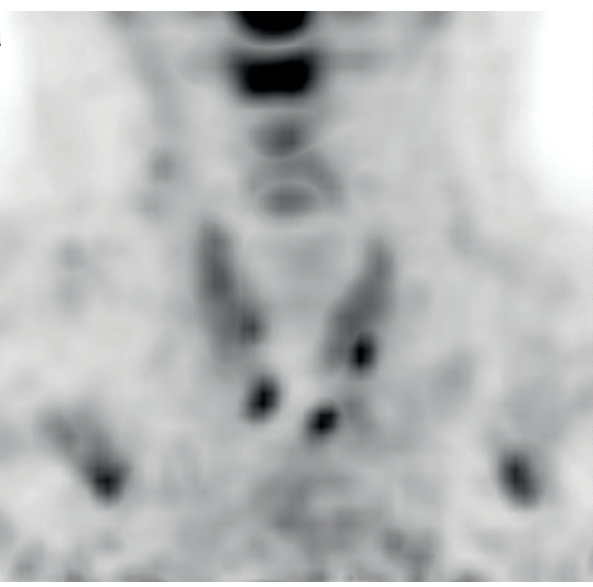

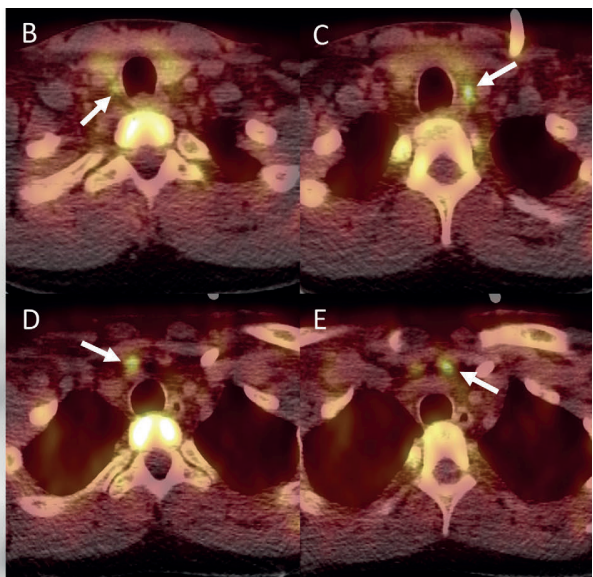

Figure 1. A 27-year-old man with chronic renal failure and tertiary hyperparathyroidism was referred for subtotal parathyroidectomy and received ${ }^{18} \mathrm{~F}$-fluorocholine $\left({ }^{18} \mathrm{~F}-\mathrm{FCH}\right) \mathrm{PET} / \mathrm{CT}$ for localization of hyperfunctioning parathyroid tissue. The scan was acquired 60 minutes after IV administration of $150 \mathrm{MBq}$ (4.1 $\mathrm{mCi})^{18} \mathrm{~F}-\mathrm{FCH}$. The images showed 4 enlarged parathyroid glands with elevated choline uptake, indicative for hyperfunctioning glands (A, MIP coronal thin-reconstruction with a slice thickness of $5 \mathrm{~cm}$. B-E, axial $\mathrm{PET/CT}$ fusion images with right superior, left superior, right inferior, and left inferior parathyroid gland, respectively).
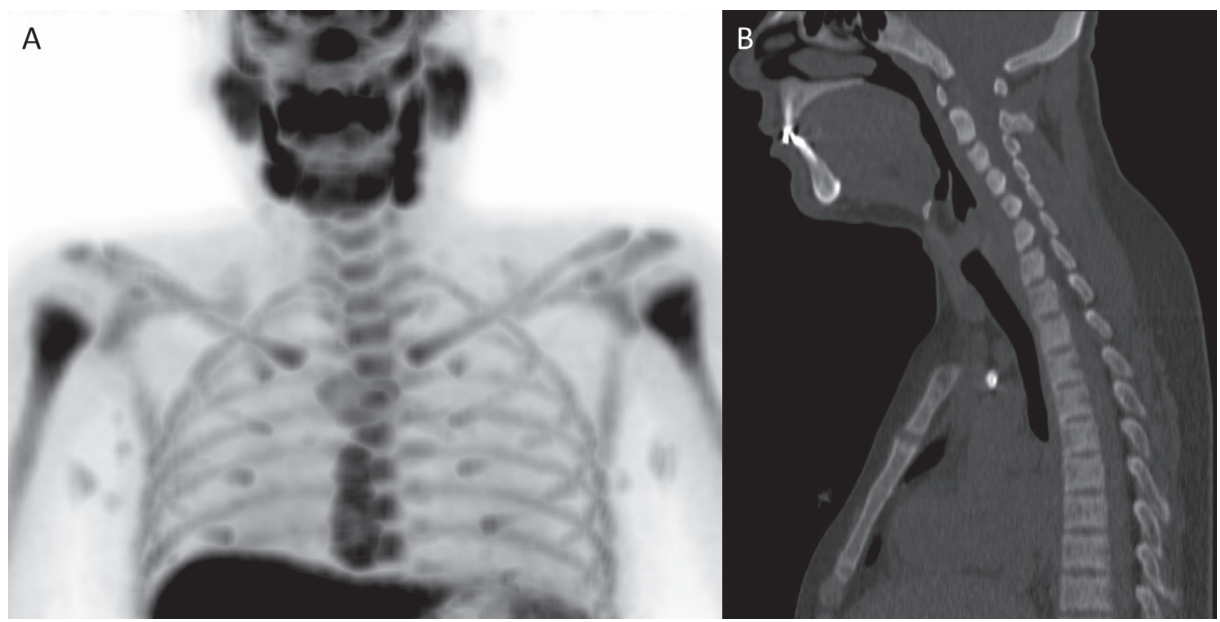

Figure 2. ${ }^{18} \mathrm{~F}$-fluorocholine PET MIP image (A) showing intense tracer uptake in the bones, especially the jaw, distal ends of long bones, and costochondral junctions (rachitic rosary bead configuration), typical for osteodystrophy. Physiological uptake can be seen in the salivary glands, liver, spleen, and some axillary lymph nodes. Furthermore, the sagittal view of the low-dose CT, used for attenuation correction, demonstrates increased osteosclerosis (B). 


\section{References}

1. Thanseer N, Bhadada SK, Sood A, et al. Comparative effectiveness of ultrasonography, ${ }^{99 \mathrm{mTC}-}$ sestamibi, and ${ }^{18} \mathrm{~F}$-fluorocholine PET/CT in detecting parathyroid adenomas in patients with primary hyperparathyroidism. Clin Nucl Med 2017;42:e491-e497.

2. Beheshti $M$, Hehenwarter L, Paymani Z, et al. ${ }^{18} \mathrm{~F}$-fluorocholine PET/CT in the assessment of primary

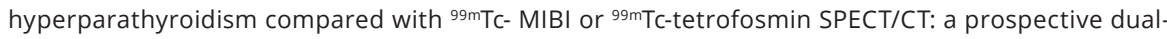
centre study in 100 patients. Eur J Nucl Med Mol Imaging 2018;45:1762-1771.

3. Karipineni F, Sahli Z, Somervell H, et al. Are preoperative sestamibi scans useful for identifying ectopic parathyroid glands in patients with expected multigland parathyroid disease? Surgery 2018;163:35-41.

4. Moe S, Drueke T, Cunningham J, et al. Definition, evaluation, and classification of renal osteodystrophy: a position statement from Kidney Disease: Improving Global Outcomes (KDIGO). Kidney Int 2006;69:1945-1953.

5. Hruska KA, Sugatani T, Agapova O, et al. The chronic kidney disease - mineral bone disorder (CKDMBD): advances in pathophysiology. Bone 2017;100:80-86.

6. de Graaf P, Schicht IM, Pauwels EK, et al. Bone scintigraphy in renal osteodystrophy. I Nucl Med 1978;19:1289-1296.

7. Ghesani N, Jung J, Patel S, et al. Superscan caused by renal osteodystrophy: observed on ${ }^{18}$ F FDG PET/ CT scan. Indian J Nucl Med 2013;28:251-252.

8. Lawal I, Ankrah A, Ololade K, et al. Renal osteodystrophy presenting as a metabolic superscan on F-18 FDG PET/CT: a case report. Medicine (Baltimore) 2017;96:e8471.

9. Reczek J, Elgazzar A. Prominent Tc-99m MIBI skeletal uptake in renal osteodystrophy: a possible role for whole-body scanning. Clin Nucl Med 2003;28:775-777.

10. Witteveen JE, van Thiel S, Romijn JA, et al. Hungry bone syndrome: still a challenge in the post-operative management of primary hyperparathyroidism: a systematic review of the literature. Eur J Endocrinol 2013;168:R45-R53. 


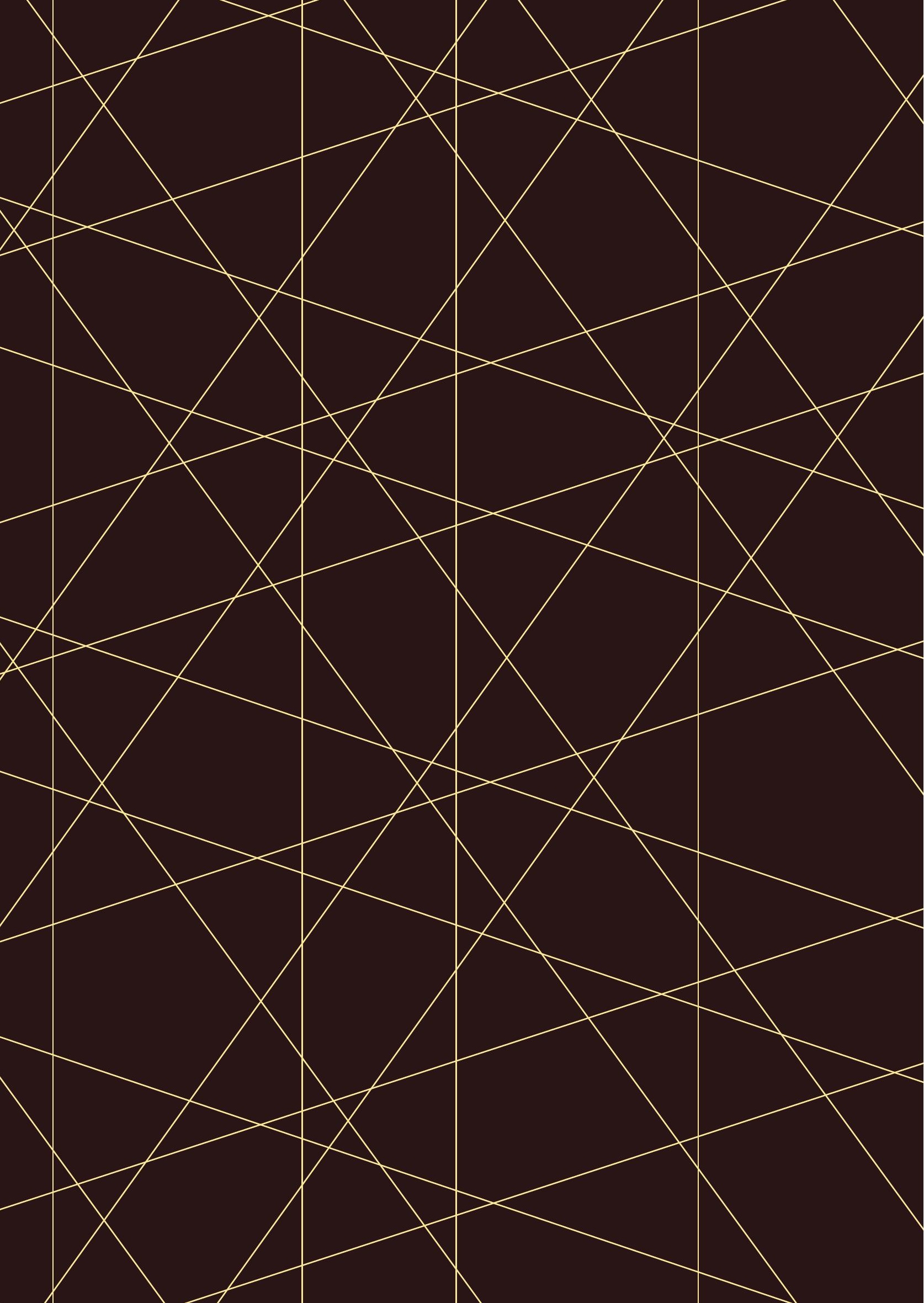




\section{PART 3}

Clinical aspects of ${ }^{18} \mathrm{~F}$-fluorocholine PET/CT 


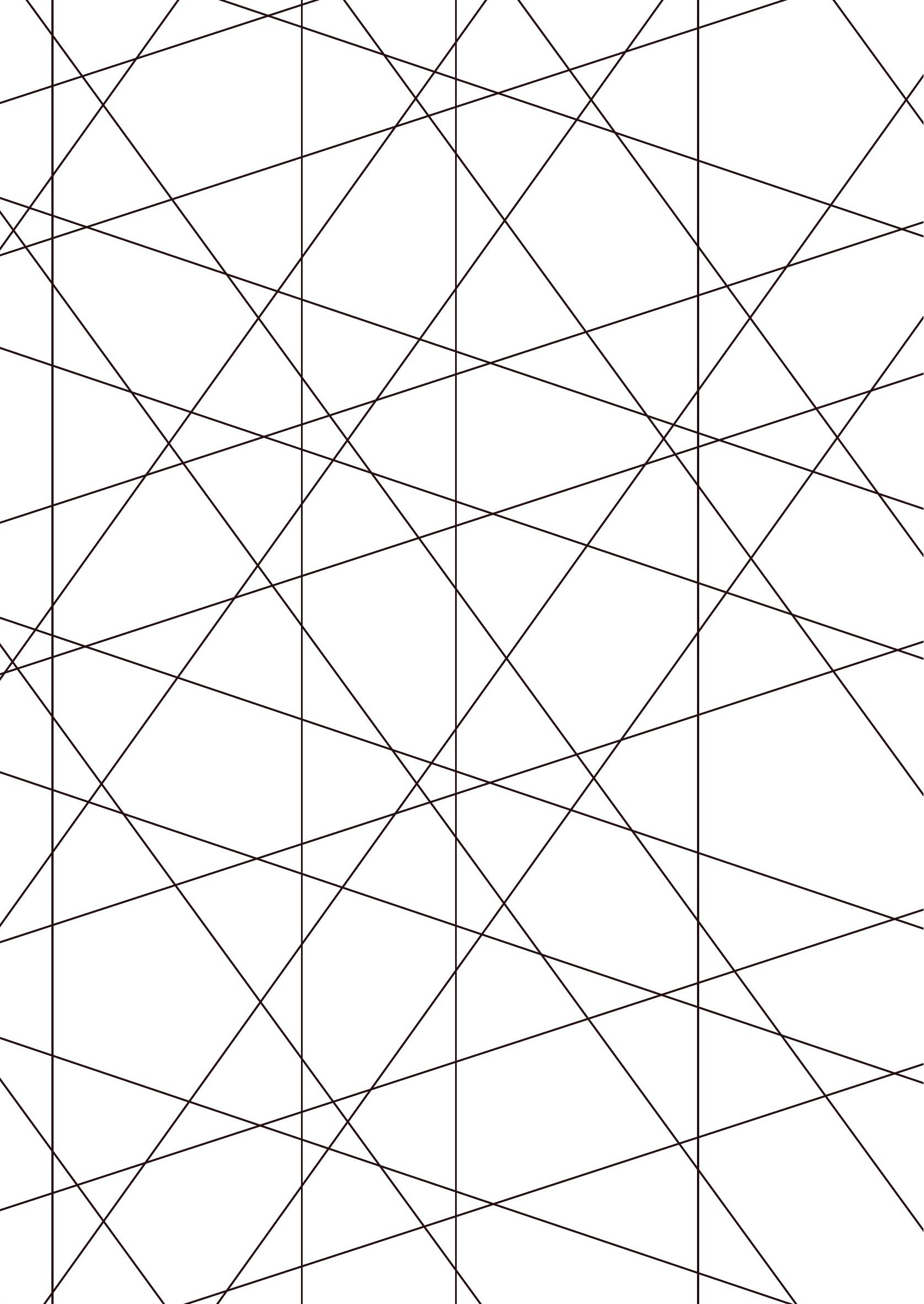




\section{CHAPTER 5}

Parathyroid imaging with ${ }^{18} \mathrm{~F}$-fluorocholine $\mathrm{PET} / \mathrm{CT}$ as a first-line imaging modality in primary hyperparathyroidism: a retrospective cohort study

Wouter A.M. Broos

Maurits Wondergem

Remco J.J. Knol

Friso M. van der Zant

European Journal of Nuclear Medicine and

Molecular Imaging Research

2019;9(1):72 


\section{Abstract}

Background: ${ }^{18} \mathrm{~F}$-fluorocholine $\left({ }^{18} \mathrm{~F}-\mathrm{FCH}\right) \mathrm{PET} / \mathrm{CT}$ is a promising technique for visualizing hyperfunctioning parathyroid glands in hyperparathyroidism. It is still under debate whether to use this technique as a first-line imaging modality or to use it when conventional techniques such as ${ }^{99 \mathrm{mT}} \mathrm{Tc}$-sestamibi scintigraphy or ultrasonography are inconclusive. This study evaluates ${ }^{18} \mathrm{~F}-\mathrm{FCH}$ PET/CT as a first-line modality.

Methods: Patients with primary hyperparathyroidism, referred between June 2015 and December 2018 for ${ }^{18} \mathrm{~F}-\mathrm{FCH}$ PET/CT as a first-line imaging method, were included in this study. Baseline characteristics, clinical data, scan results, and type of treatment were recorded. The rate of correct detection was calculated on a per patient-based and a per lesion-based analysis. The reference standard comprised histopathological results, intraoperative response to parathyroidectomy, and clinical follow-up.

Results: Two hundred and seventy-one patients were included, of which 139 patients underwent parathyroidectomy, 48 were treated with calcimimetics, and 84 patients received further follow-up without active treatment. In the surgically treated group, a single adenoma was suspected in 127 scans, double adenoma in three scans, and one scan showed evidence of three hyperfunctioning glands. In eight scans, no lesions were visualized. A total of 154 parathyroid glands were surgically removed. The rate of correct detection was calculated at $96 \%$ and $90 \%$, on a per patient-based and per lesion-based analysis, respectively.

Conclusion: This retrospective study in a large cohort shows high detection rates of ${ }^{18} \mathrm{~F}-\mathrm{FCH} \mathrm{PET} / \mathrm{CT}$ in primary hyperparathyroidism, which is in accordance to literature. The use of ${ }^{18} \mathrm{~F}-\mathrm{FCH}$ PET/CT as a first-line imaging modality in preoperative planning of parathyroid surgery may therefore be a suitable choice. 


\section{Introduction}

Primary hyperparathyroidism is a relatively common endocrine disorder, which develops as a result of autonomous overproduction of parathyroid hormone (PTH) by parathyroid glands. In most cases, the disease is caused by a single parathyroid adenoma (89\%) and less commonly by parathyroid hyperplasia (6\%), double parathyroid adenoma (4\%), or parathyroid carcinoma (<1\%).[1] Clinical presentation is heterogeneous. Asymptomatic disease is the most frequent presentation in Western countries where routine laboratory testing is common.[2,3] Kidney stones occur in 15-20\% and a second important clinical feature is bone loss with associated fracture risk.[4] Primary hyperparathyroidism is routinely treated with parathyroidectomy, which still is the only definitive management. In surgical treatment of hyperparathyroidism, precise preoperative localization of hyperfunctioning parathyroid tissue has become increasingly important, due to a shift from bilateral neck exploration to minimally invasive parathyroidectomy in the past decades.[5]

The current reference method for localization of hyperfunctioning parathyroid glands is ${ }^{99 m} \mathrm{mc}$-sestamibi scintigraphy, with reported detection rates of $84-88 \% .[6,7]$ In recent years, PET/CT imaging with ${ }^{18} \mathrm{~F}$-fluorocholine $\left({ }^{18} \mathrm{~F}-\mathrm{FCH}\right)$ has gained interest for parathyroid imaging. This technique provides higher spatial resolution, shorter scanning time, and less radiation dose and has provided promising results with detection rates exceeding $90 \%$.[8,9] Whether to use this technique as a first-line imaging modality, or to use it when conventional techniques are inconclusive, is still under discussion. In this study, the use of ${ }^{18} \mathrm{~F}-\mathrm{FCH}$ PET/CT as a first-line modality was evaluated. 


\section{Methods}

\section{Patient selection}

Patients who received ${ }^{18} \mathrm{~F}-\mathrm{FCH}$ PET/CT for analysis of primary hyperparathyroidism from June 2015 through December 2018 were included in this study. Primary hyperparathyroidism, according to the American Association of Endocrine Surgeons Guidelines, was defined as the persistent elevation of total serum calcium levels with corresponding elevated or inappropriately normal PTH levels, or high PTH levels and normal serum calcium levels.[10] Patients with familial hypocalciuric hypercalcemia were excluded.

When patients were referred more than once for ${ }^{18} \mathrm{~F}-\mathrm{FCH}$ PET/CT imaging, all scans except for the most recent scan before surgery were excluded. When patients received multiple scans but no surgery, the follow-up scans were excluded. Patients who were on the waiting list for parathyroidectomy were also excluded. All patient data, including baseline characteristics and scan results, were entered into a database. Additional follow-up data were retrieved from the electronic patient records.

\section{Informed consent}

All patients gave written informed consent for use of their anonymized data for scientific purposes. Besides the standard imaging protocol and clinical management, no additional measurements or actions affecting the patient were performed. The study was approved by the institutional research department and performed in accordance with the Declaration of Helsinki. Approval of the local ethical committee for the present study was not necessary since the study does not fall within the scope of the Dutch Medical Research Involving Human Subjects Act (section 1.b WMO, 26th February 1998).

\section{Scan acquisition}

PET/CT images were acquired on a Siemens Biograph-16 TruePoint PET/CT camera (Siemens Healthcare, Knoxville, TN, USA). Dual-time-point images were acquired at 5 min and 60 min after intravenous injection of approximately $150 \mathrm{MBq}{ }^{18} \mathrm{~F}-\mathrm{FCH}$, ranging from the temporomandibular joint to the diaphragm. Images were acquired at $480 \mathrm{~s}$ per bed position with matrix size $256 \times 256$ and low-dose CT for attenuation correction using a tube current of 40-80 mAs at 100-120 kV with CARE Dose 4D dose modulation, collimation of $24 \times 1.2 \mathrm{~mm}$ and a pitch of 0.95 . PET images were reconstructed with an iterative 3D method using 5 iterations, 8 subsets, and a Gaussian filter. Interpretation of the images and quantitative measurements were done with dedicated software (Syngo. via, Siemens Medical Solutions, Malvern, PA, USA). Patient preparation consisted of 
hydration with $1 \mathrm{~L}$ water and, if applicable, discontinuation of colchicine $48 \mathrm{~h}$ prior to ${ }^{18} \mathrm{~F}-\mathrm{FCH}$ administration. Discontinuation of calcimimetic drugs or other medications was not required.

\section{Image interpretation}

Imaging results were prospectively recorded in a database after reading of the scan. Each ${ }^{18} \mathrm{~F}-\mathrm{FCH}$ PET/CT was scored as negative, equivocal, or positive for hyperfunctioning parathyroid glands. In the presence of well-defined focal ${ }^{18} \mathrm{~F}$-FCH uptake found in typical locations, the scan was considered positive (Fig. 1). A normal distribution of ${ }^{18} \mathrm{~F}-\mathrm{FCH}$ or atypical tracer distribution with an alternative explanation rather than hyperfunctioning parathyroid tissue defined the scan as negative. Equivocal scans were discussed in a consensus meeting with three nuclear medicine physicians (W.B., F.Z., and M.W.), blinded from other patient data, and scored as positive or negative. The location and number of foci were also entered into the database. For scans positive for parathyroid adenoma,

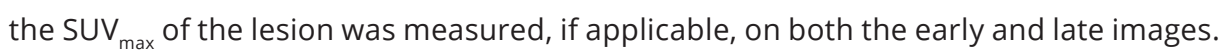

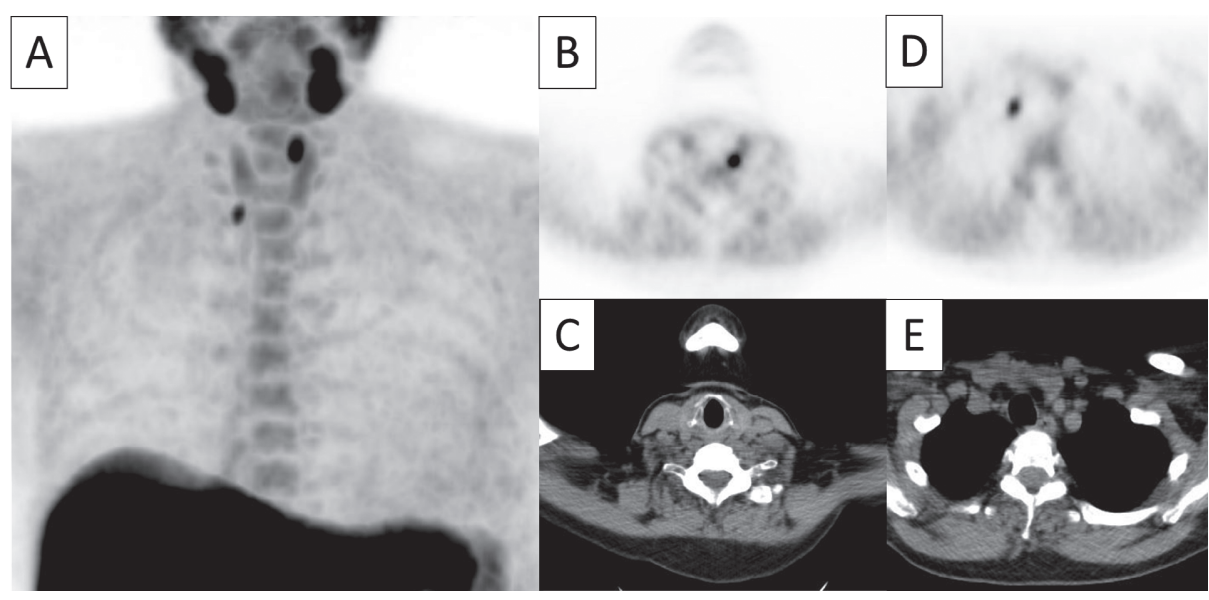

Figure 1. Example of a positive ${ }^{18} \mathrm{~F}$-fluorocholine $\mathrm{PET} / \mathrm{CT}$ representing a double parathyroid adenoma. Serum calcium level in this patient was $2.61 \mathrm{mmol} / \mathrm{L}$ with a parathyroid hormone level of $27.7 \mathrm{pmol} / \mathrm{L}$. The maximum-intensity projection image (A) shows two foci of intense choline uptake in the neck and physiological uptake in the salivary glands, thyroid gland, liver, spleen, and bone marrow. Axial views of PET and CT show foci suspicious for parathyroid adenomas located dorsocranial to the left thyroid lobe (B, C, dimensions $7 \times 5 \mathrm{~mm}$ and SUV ${ }_{\max }$ of 8.7) and inferior to the right thyroid lobe (D, E, dimensions $6 \times$ $4 \mathrm{~mm}$ and SUV max $_{\text {of 5.6). }}$. 


\section{Statistical analysis}

Histopathological findings in combination with intraoperative results (gland inspection by the surgeon and intraoperative PTH monitoring) and clinical follow-up data were used as the reference standard. The rate of correct detection was calculated as the number of true positives divided by the sum of true positives and false negatives. Detection rates were calculated in a per patient-based and per lesion-based analysis. Pearson's correlation coefficient was calculated to evaluate the correlation between parameters

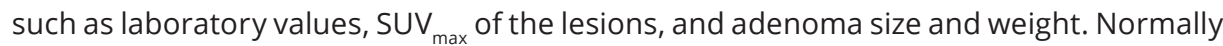
distributed continuous data were expressed as mean \pm standard deviation (SD) and range. Non-continuous data were expressed as numbers with percentages. The analysis was carried out using the Statistical Package for Social Sciences 20 (IBM SPSS Statistics, Chicago, IL, USA).

\section{Results}

A total of $294{ }^{18} \mathrm{~F}-\mathrm{FCH}$ PET/CT scans were performed for analysis of primary hyperparathyroidism during the studied period. Eight scans were excluded because there was a more recent scan available before surgery. Three scans were excluded because these were follow-up scans. Another 12 patients were excluded since they were lost-to-follow-up due to surgery in another hospital, death before surgery, or because patients were still on the waiting list for parathyroidectomy at the time of analysis. A total of 271 patients were included in this study. All ${ }^{18} \mathrm{~F}-\mathrm{FCH}$ PET/CT scans were performed as first-line imaging for localization of hyperfunctioning parathyroid glands as is the standard of care in our institution. Patients were referred from two different hospitals, while imaging and surgery were performed in the same institution.

Patient characteristics and laboratory values (serum calcium, PTH, phosphate, and 24-h urinary calcium levels) are presented in Table 1. Five patients were known with multiple endocrine neoplasia syndrome (three patients with MEN1 and two patients with MEN2a) and 17 patients had lithium associated disease. Ten patients had a history of parathyroid surgery and 7 patients had a history of thyroid surgery. Of the MEN1 patients, one had previous parathyroid surgery. Both MEN2a patients had previous thyroid surgery. Symptomatic disease was present in 146 patients; 51 patients had kidney stones or nephrocalcinosis and 84 patients had osteoporosis or insufficiency fractures. Other reported symptoms, likely to be related to the disease, were musculoskeletal complaints, abdominal pains, nausea, and fatigue. 
Scans were positive for hyperfunctioning parathyroid glands in 202 patients (75\%) and negative in 69 patients (25\%), of which 16 were initially scored as equivocal (6 positives and 10 negatives after consensus). A total of 213 foci that were suspicious for hyperfunctioning parathyroid glands were detected; 23 foci (11\%) were located left superior, 74 (35\%) left inferior, 22 (10\%) right superior, 85 (40\%) right inferior, and nine (4\%) glands were ectopically located. Parathyroidectomy was performed in 139 patients (51\%), 48 patients (18\%) were treated with calcimimetics, and 84 patients (31\%) received no active treatment. Of the pharmacologically treated patients, most received $30 \mathrm{mg}$ of Cinacalcet per day $(n=36)$, and the other patients received dosages of $7.5 \mathrm{mg}(n=1), 60 \mathrm{mg}(n=8)$, $90 \mathrm{mg}(\mathrm{n}=1)$, or $120 \mathrm{mg}(\mathrm{n}=2)$ per day. Demographics and laboratory values of each subgroup are listed in Table 1. Mean follow-up was $22 \pm 11$ months (range 3-43 months).

Ten patients showed evidence of multiple active glands; nine patients with two foci and one patient with three foci. Of the patients with two foci, only three underwent surgery. In two of those patients, the two corresponding parathyroid glands were removed and histopathologically confirmed to be adenomas. In the other patient, only one parathyroid adenoma was found at surgery after which the patient was cured indicating a falsepositive finding on the scan. The patient with three foci, a patient with MEN2a syndrome, underwent surgery as well. In this patient, two out of the three foci were confirmed to be adenomas. The other MEN patients had no signs of multiglandular disease on PET/CT.

The subgroup with patients that underwent surgery was further analyzed. Cure was achieved in 136 patients. In two patients, no parathyroid adenoma was found intraoperatively, both with a negative scan, and in one patient, a parathyroid adenoma was removed but elevated PTH persisted during follow-up. In all other patients, intraoperative PTH significantly decreased to normal values or showed at least a 50\% reduction and no recurrence occurred during follow-up. In these patients, also serum calcium levels normalized without relapse during follow-up. The type of surgery was minimally invasive in $75 \%$ of the procedures, and a standard Kocher incision was performed in the other $25 \%$, in some cases combined with bilateral exploration. A total of 154 parathyroid glands were removed. Fourteen (9\%) of the removed glands were located left superior, 57 (37\%) left inferior, 15 (10\%) right superior, 64 (41\%) right inferior, and four (3\%) glands were ectopically located. Histopathological results revealed adenoma in 133 of the removed glands (86\%) and hyperplasia in five (3\%), and in 16 cases (10\%), the pathology report confirmed that the removed tissue contained parathyroid gland without further specification. When serum PTH decreased after removal of the glands, these were considered as hyperfunctioning parathyroid glands. The mean weight of the removed glands was $1.06 \mathrm{~g}$ (SD 2.43, 95\% Cl 0.62-1.49), the mean maximum length was 1.60 $\mathrm{cm}(\mathrm{SD} 0.95,95 \% \mathrm{Cl} 1.43-1.76)$, and the mean volume was $1.74 \mathrm{~cm}^{3}$ (SD 4.79, 95\% Cl 0.81-2.68). 


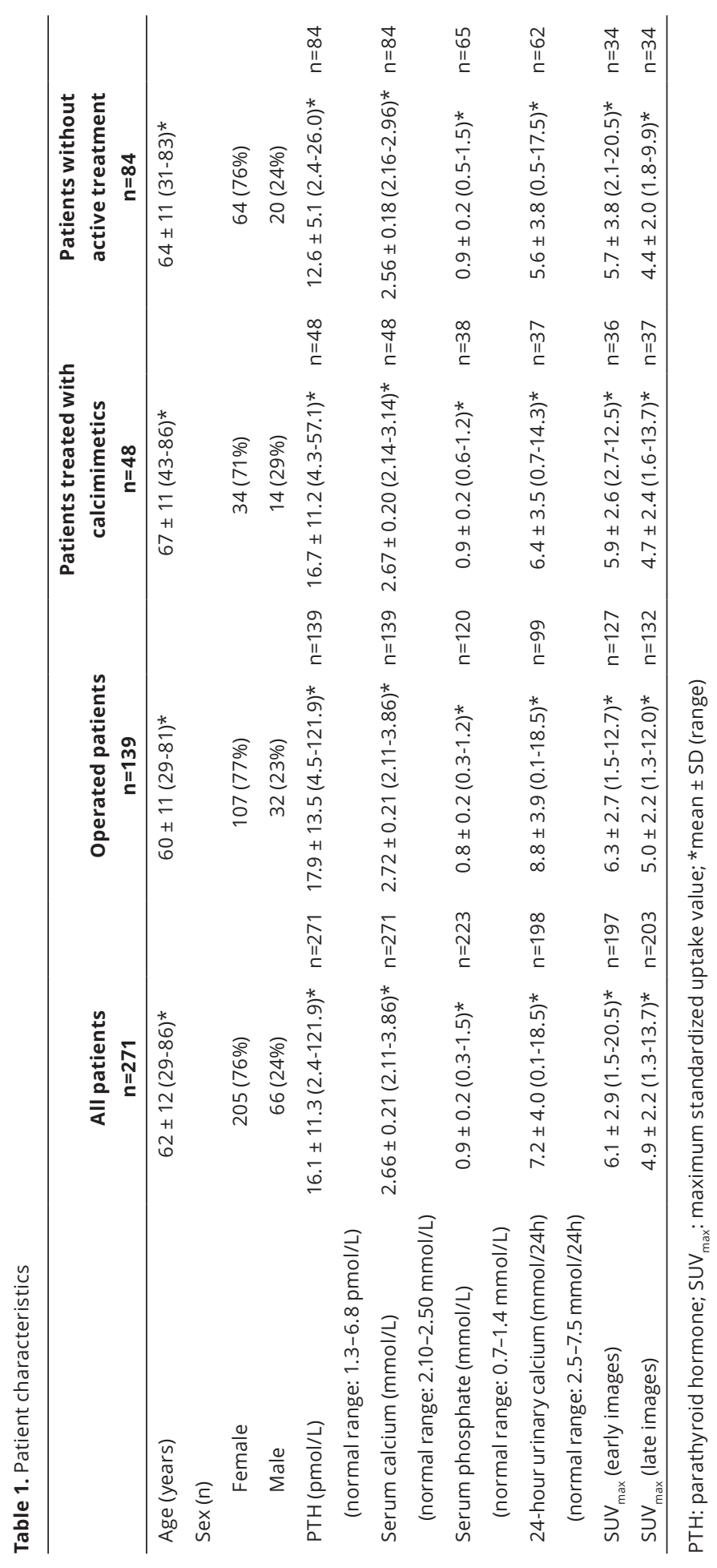


In the subgroup of 139 operated patients, 131 scans were positive for hyperfunctioning parathyroid tissue. These could all be designated as true positives after correlation with surgical and histopathological data. Six of the eight negative scans proved to be false negatives during surgery. This resulted in a calculated detection rate of $96 \%$ (95\% Cl 91-98\%), on a per patient-based analysis. In the per lesion-based analysis, 133 of the 154 removed glands were correctly detected on the scans. There were 15 histopathologically proven parathyroid adenomas that were not detected on the scans. Calculated per lesion-based detection rate was therefore 90\% (95\% Cl 84-94\%). These results are summarized in Table 2 . Detection of hyperfunctioning glands was lower in the non-operatively treated subgroups; 38 out of 48 (79\%) in the subgroup treated with calcimimetics, and only 33 out of 84 (39\%) in the subgroup receiving no active treatment. Because no reference standard was available in those patients, the rate of correct detection could not be calculated for these subgroups.

Table 2. Scan performance

\begin{tabular}{lcccc}
\hline & Total & True positive & False negative & Correct detection rate (95\% CI) (\%) \\
\hline Patient-based & 139 & 131 & 6 & $96(91-98)$ \\
Lesion-based & 154 & 133 & 15 & $90(84-94)$ \\
\hline
\end{tabular}

The SUV ${ }_{\max }$ was measured on 197 of the early images and on 203 of the late images. The mean SUV ${ }_{\max }$ on the early images was 6.1 and decreased to a mean SUV $V_{\max }$ of 4.9 on the late images, which is in line with our previous study on this matter.[11] The uptake values for each subgroup are shown in Table 1. These measurements, along with adenoma sizes and weight which were retrieved from the pathology reports, were correlated with laboratory values by calculating the Pearson's correlation coefficient $(r)$. Weak to very weak correlations were found between SUV and laboratory values ( $r$ values of 0.12-0.31) and size or weight ( $r$ values of 0.17-0.38). There was a moderate correlation between the serum calcium level and adenoma weight $(r=0.40)$ and volume $(r=0.41)$, and a strong correlation was found between the PTH level and weight $(r=0.69)$ and volume $(r=0.72)$. Weight and size were only weakly correlated with serum phosphate levels ( $r$ values of -0.24 and -0.25 , respectively). No significant $r$ could be calculated between 24 -h urinary calcium levels and other parameters. 


\section{Discussion}

In this study, the performance of ${ }^{18} \mathrm{~F}-\mathrm{FCH}$ PET/CT as a first-line imaging method was investigated. Most studies that have been published on the use of ${ }^{18} \mathrm{~F}-\mathrm{FCH}$ PET/CT in hyperparathyroidism used this scan as a second-line imaging method when conventional imaging methods, such as ${ }^{99 m T c-s e s t a m i b i ~ s c i n t i g r a p h y ~ a n d ~ u l t r a s o n o g r a p h y, ~ w e r e ~}$ inconclusive, or when previous surgery had failed.[12-19] Fewer studies were published on the performance of ${ }^{18} \mathrm{~F}-\mathrm{FCH}$ PET/CT as a first-line imaging modality, with reported sensitivities ranging between $89 \%$ and 100\%.[20-23] The results from the present study, with a detection rate of $96 \%$ and $90 \%$, on a per patient-based and a per lesion-based respectively, are in line with these earlier studies.

In this study, the detection rate was calculated as the number of true positives divided by the total of true positives and false negatives. The term detection rate was used instead of sensitivity, because of the highly selected patient population which only contained patients with biochemically proven primary hyperparathyroidism. Additionally, the purpose of imaging is not to confirm or exclude the disease, but to detect and localize the diseased parathyroid glands. Frequently, true negative numbers are not available, because acquiring histopathological proof of intraoperatively normal-looking glands is not recommended and in minimally invasive parathyroidectomy, the other parathyroid glands are not inspected.[10] In this study, both the per patient-based as well as the per lesion-based detection rate were calculated. The use of the lesion-based detection rate is most appropriate, because this number represents the correct localization of the gland, which is essential in preoperative planning. The main strength of this study is the large cohort size, including a substantial group of patients that underwent parathyroidectomy. However, $18 \%$ of the patients were treated with medication and 31\% received further follow-up without active treatment, and for this part of the cohort, no detection rate could be calculated because of the lack of a reference standard. This subjects the analysis of the operated patient group to selection bias and possibly leads to an overestimation of the detection rates, which are, nevertheless, in accordance with the literature. Prospective trials could reduce this selection bias.

Besides the excellent detection rates of ${ }^{18} \mathrm{~F}-\mathrm{FCH}$ PET/CT, there are several other benefits of this modality above conventional scintigraphy. ${ }^{18} \mathrm{~F}-\mathrm{FCH} \mathrm{PET} / \mathrm{CT}$ has shorter acquisition times and lower radiation dose, and there is no need to stop calcimimetic drugs. PET/CT scanners have become more commonly available, and the tracer is suitable to be shipped due to the relatively long half-life of $110 \mathrm{~min}$. In general, PET/CT is more expensive than conventional scintigraphy; however, costs can be reduced if an on-site cyclotron 
is available or when a hospital can act as a referral center with frequent use of the technique. Also, the cost of radiopharmaceuticals could be reduced with more sensitive scanners and more sophisticated software. Cost-effectiveness was not evaluated in this study and has not been investigated in earlier studies but will be relevant for the decision whether to use ${ }^{18} \mathrm{~F}-\mathrm{FCH}$ PET/CT as the first-line imaging method instead of conventional scintigraphy.

It is expected that detection rates will improve with new generation PET/CT scanners. Present digital systems have effective sensitivities up to 98.4 counts per second (cps) per $\mathrm{kBq}$, compared to $9 \mathrm{cps} / \mathrm{kBq}$ for the analog Siemens TruePoint PET/CT, used in this study. This is especially relevant for the detection of small lesions such as parathyroid glands. Besides ${ }^{18} \mathrm{~F}-\mathrm{FCH} \mathrm{PET} / \mathrm{CT}$, another promising imaging technique is contrast-enhanced $\mathrm{CT}$ acquired at multiple time points (4D-CT). Although the performance of $C T$ is good, the main disadvantage is the higher radiation dose.[24] A pilot study demonstrated that ${ }^{18} \mathrm{~F}-\mathrm{FCH}$ PET/CT is comparable in performance to 4D-CT [25], a recent study showed that the combination of ${ }^{18} \mathrm{~F}-\mathrm{FCH}$ PET with 4D-CT was of added value [18], and a third study concluded that 4D-CT appears as a confirmatory imaging modality.[19] Furthermore, an interesting development is the use of ${ }^{18} \mathrm{~F}-\mathrm{FCH}$ PET/MRI, with the reduction of radiation dose and increased soft-tissue resolution.[16,26,27]

In our institution, ${ }^{18} \mathrm{~F}-\mathrm{FCH}$ PET/CT is performed as a first-line imaging method in the routine clinical work-up of hyperparathyroid patients. No ${ }^{99 m T c-s e s t a m i b i ~ s c i n t i g r a p h y, ~}$ $\mathrm{CT}$, or MRI was performed before ${ }^{18} \mathrm{~F}-\mathrm{FCH}$ PET/CT in these patients. Also, ultrasound is no prerequisite in our institution. Only patients with primary hyperparathyroidism were included in this study; therefore, no conclusions can be drawn on the use of ${ }^{18} \mathrm{~F}-\mathrm{FCH}$ PET/CT as a first-line imaging method in secondary of tertiary hyperparathyroidism. The group of patients in this study was heterogeneous, representing the population in our institution. Both symptomatic and asymptomatic patients were represented and patients with typical elevated serum calcium and PTH levels but also with normocalcemic hyperparathyroidism were included.

In the group of patients who underwent surgery, high detection rates were found, and it can be concluded that in this setting, the ${ }^{18} \mathrm{~F}-\mathrm{FCH}$ PET/CT is a useful method to guide the surgeon. A noteworthy part of the studied group, however, did not undergo surgery. In this group (treated with medication or without active treatment), the exact value of the ${ }^{18} \mathrm{~F}-\mathrm{FCH}$ PET/CT cannot be assessed, since the influence of the scan on further treatment of the patient and clinical outcome is unclear. One possible explanation for the relatively high rate of non-operated patients is that ${ }^{18} \mathrm{~F}-\mathrm{FCH}$ PET/CT is performed rather early in the 
diagnostic process in our institution. Regularly, the patients are referred for ${ }^{18} \mathrm{~F}-\mathrm{FCH}$ PET/ CT by the endocrinologist and are referred for surgical intervention at a later time point. However, the general consensus is that imaging of parathyroid glands is performed for preoperative localization in patients for which operation is already indicated.[10] The value of ${ }^{18} \mathrm{~F}-\mathrm{FCH} \mathrm{PET} / \mathrm{CT}$ or other parathyroid imaging techniques to prove or rule out parathyroid disease is unknown.

\section{Conclusion}

This retrospective cohort study shows high detection rates of ${ }^{18} \mathrm{~F}-\mathrm{FCH} \mathrm{PET} / \mathrm{CT}$ in primary hyperparathyroidism, which is in accordance with literature. The use of ${ }^{18} \mathrm{~F}-\mathrm{FCH}$ PET/CT as a first-line imaging modality in preoperative planning of parathyroid surgery seems an appropriate choice; however, cost-effectiveness analyses are warranted. 


\section{References}

1. Ruda JM, Hollenbeak CS, Stack BC Jr. A systematic review of the diagnosis and treatment of primary hyperparathyroidism from 1995 to 2003. Otolaryngol Head Neck Surg 2005;132:359-372.

2. Bilezikian JP, Bandeira L, Khan A, Cusano NE. Hyperparathyroidism. Lancet 2018;391:168-178.

3. Abood A, Vestergaard P. Increasing incidence of primary hyperparathyroidism in Denmark. Dan Med J 2013;60:A4567.

4. Silverberg SJ, Clarke BL, Peacock M, Bandeira F, Boutroy S, Cusano NE, et al. Current issues in the presentation of asymptomatic primary hyperparathyroidism: proceedings of the Fourth International Workshop. J Clin Endocrinol Metab 2014;99:3580-3594.

5. Sackett WR, Barraclough B, Reeve TS, Delbridge LW. Worldwide trends in the surgical treatment of primary hyperparathyroidism in the era of minimally invasive parathyroidectomy. Arch Surg 2002;137:1055-1059.

6. Wong KK, Fig LM, Gross MD, Dwamena BA. Parathyroid adenoma localization with ${ }^{99 m} \mathrm{Tc}$-sestamibi SPECT/CT: a meta-analysis. Nucl Med Commun 2015;36:363-375.

7. Treglia G, Sadeghi R, Schalin-Jantti C, Caldarella C, Ceriani L, Giovanella L, et al. Detection rate of ${ }^{99 \mathrm{~m} T C-}$ MIBI single photon emission computed tomography (SPECT)/CT in preoperative planning for patients with primary hyperparathyroidism: a meta-analysis. Head Neck 2016;38(Suppl 1):E2159-E2172.

8. Broos WAM, van der Zant FM, Knol RJJ, Wondergem M. Choline PET/CT in parathyroid imaging: a systematic review. Nucl Med Commun 2019;40:96-105.

9. Treglia G, Piccardo A, Imperiale A, Strobel K, Kaufmann PA, Prior JO, et al. Diagnostic performance of choline PET for detection of hyperfunctioning parathyroid glands in hyperparathyroidism: a systematic review and meta-analysis. Eur J Nucl Med Mol Imaging 2018;46:751-765.

10. Wilhelm SM, Wang TS, Ruan DT, Lee JA, Asa SL, Duh QY, et al. The American Association of Endocrine Surgeons guidelines for definitive management of primary hyperparathyroidism. JAMA Surg 2016;151:959-968.

11. Broos $\mathrm{W}$, Wondergem $\mathrm{M}$, van der Zant $\mathrm{F}$, Knol R. Dual-time-point ${ }^{18} \mathrm{~F}$-fluorocholine PET/CT in parathyroid imaging. J Nucl Med 2019;60:1605-1610.

12. Michaud L, Balogova S, Burgess A, Ohnona J, Huchet V, Kerrou K, et al. A pilot comparison of

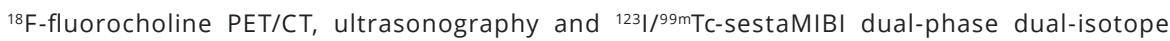
scintigraphy in the preoperative localization of hyperfunctioning parathyroid glands in primary or secondary hyperparathyroidism: influence of thyroid anomalies. Medicine (Baltimore) 2015;94:e1701.

13. Kluijfhout WP, Vorselaars WM, van den Berk SA, Vriens MR, Borel Rinkes IH, Valk GD, et al. Fluorine-18 fluorocholine PET-CT localizes hyperparathyroidism in patients with inconclusive conventional imaging: a multicenter study from the Netherlands. Nucl Med Commun 2016;37:1246-1252.

14. Quak E, Blanchard D, Houdu B, Le Roux Y, Ciappuccini R, Lireux B, et al. F18-choline PET/CT guided surgery in primary hyperparathyroidism when ultrasound and MIBI SPECT/CT are negative or inconclusive: the APACH1 study. Eur J Nucl Med Mol Imaging 2017;45:658-666.

15. Fischli S, Suter-Widmer I, Nguyen BT, Muller W, Metzger J, Strobel K, et al. The significance of ${ }^{18} \mathrm{~F}$-fluorocholine-PET/CT as localizing imaging technique in patients with primary hyperparathyroidism and negative conventional imaging. Front Endocrinol (Lausanne) 2018;8:380.

16. Huber GF, Hullner M, Schmid C, Brunner A, Sah B, Vetter D, et al. Benefit of ${ }^{18} \mathrm{~F}$-fluorocholine PET imaging in parathyroid surgery. Eur Radiol 2018;28:2700-2707.

17. Araz M, Soydal C, Ozkan E, Kir MK, Ibis E, Gullu S, et al. The efficacy of fluorine-18-choline PET/CT in

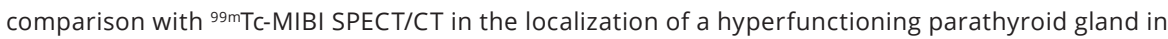
primary hyperparathyroidism. Nucl Med Commun 2018;39:989-994. 
18. Piccardo A, Trimboli P, Rutigliani M, Puntoni M, Foppiani L, Bacigalupo L, et al. Additional value of integrated ${ }^{18} \mathrm{~F}$-choline PET/4D contrast-enhanced CT in the localization of hyperfunctioning parathyroid glands and correlation with molecular profile. Eur J Nucl Med Mol Imaging 2018;46:766-775.

19. Amadou C, Bera G, Ezziane M, Chami L, Delbot T, Rouxel A, et al. ${ }^{18} \mathrm{~F}$-Fluorocholine PET/CT and parathyroid 4D computed tomography for primary hyperparathyroidism: the challenge of reoperative patients. World J Surg 2019;43:1232-1242.

20. Hocevar M, Lezaic L, Rep S, Zaletel K, Kocjan T, Sever MJ, et al. Focused parathyroidectomy without intraoperative parathormone testing is safe after pre-operative localization with ${ }^{18} \mathrm{~F}$-fluorocholine PET/CT. Eur J Surg Oncol 2017;43:133-137.

21. Thanseer N, Bhadada SK, Sood A, Mittal BR, Behera A, Gorla AKR, et al. Comparative effectiveness of ultrasonography, ${ }^{99 m T C-s e s t a m i b i, ~ a n d ~}{ }^{18} \mathrm{~F}$-fluorocholine PET/CT in detecting parathyroid adenomas in patients with primary hyperparathyroidism. Clin Nucl Med 2017;42:e491-e497.

22. Beheshti M, Hehenwarter L, Paymani Z, Rendl G, Imamovic L, Rettenbacher R, et al. ${ }^{18}$ F-fluorocholine

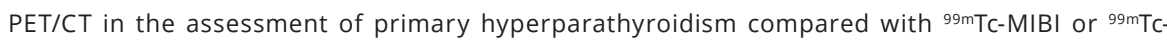
tetrofosmin SPECT/CT: a prospective dual-centre study in 100 patients. Eur J Nucl Med Mol Imaging 2018;45:1762-1771.

23. Bossert I, Chytiris S, Hodolic M, Croce L, Mansi L, Chiovato L, et al. PETC/CT with ${ }^{18}$ F-Choline localizes hyperfunctioning parathyroid adenomas equally well in normocalcemic hyperparathyroidism as in overt hyperparathyroidism. J Endocrinol Invest 2018;42:419-426.

24. Kluijfhout WP, Pasternak JD, Beninato T, Drake FT, Gosnell JE, Shen WT, et al. Diagnostic performance of computed tomography for parathyroid adenoma localization; a systematic review and meta-analysis. Eur J Radiol 2017;88:117-128.

25. Taywade SK, Damle NA, Behera A, Devasenathipathy K, Bal C, Tripathi M, et al. Comparison of ${ }^{18} \mathrm{~F}$-fluorocholine positron emission tomography/computed tomography and four-dimensional computed tomography in the preoperative localization of parathyroid adenomas-initial results. Indian J Endocrinol Metab 2017;21:399-403.

26. Alharbi AA, Alshehri FM, Albatly AA, Sah BR, Schmid C, Huber GF, et al. ${ }^{18} \mathrm{~F}$-Fluorocholine uptake of parathyroid adenoma is correlated with parathyroid hormone level. Mol Imaging Bio/ 2018;20:857-867.

27. Khafif A, Masalha M, Landsberg R, Domachevsky L, Bernstine H, Groshar D, et al. The role of F18fluorocholine positron emission tomography/magnetic resonance imaging in localizing parathyroid adenomas. Eur Arch Otorhinolaryngol 2019;276:1509-1516. 


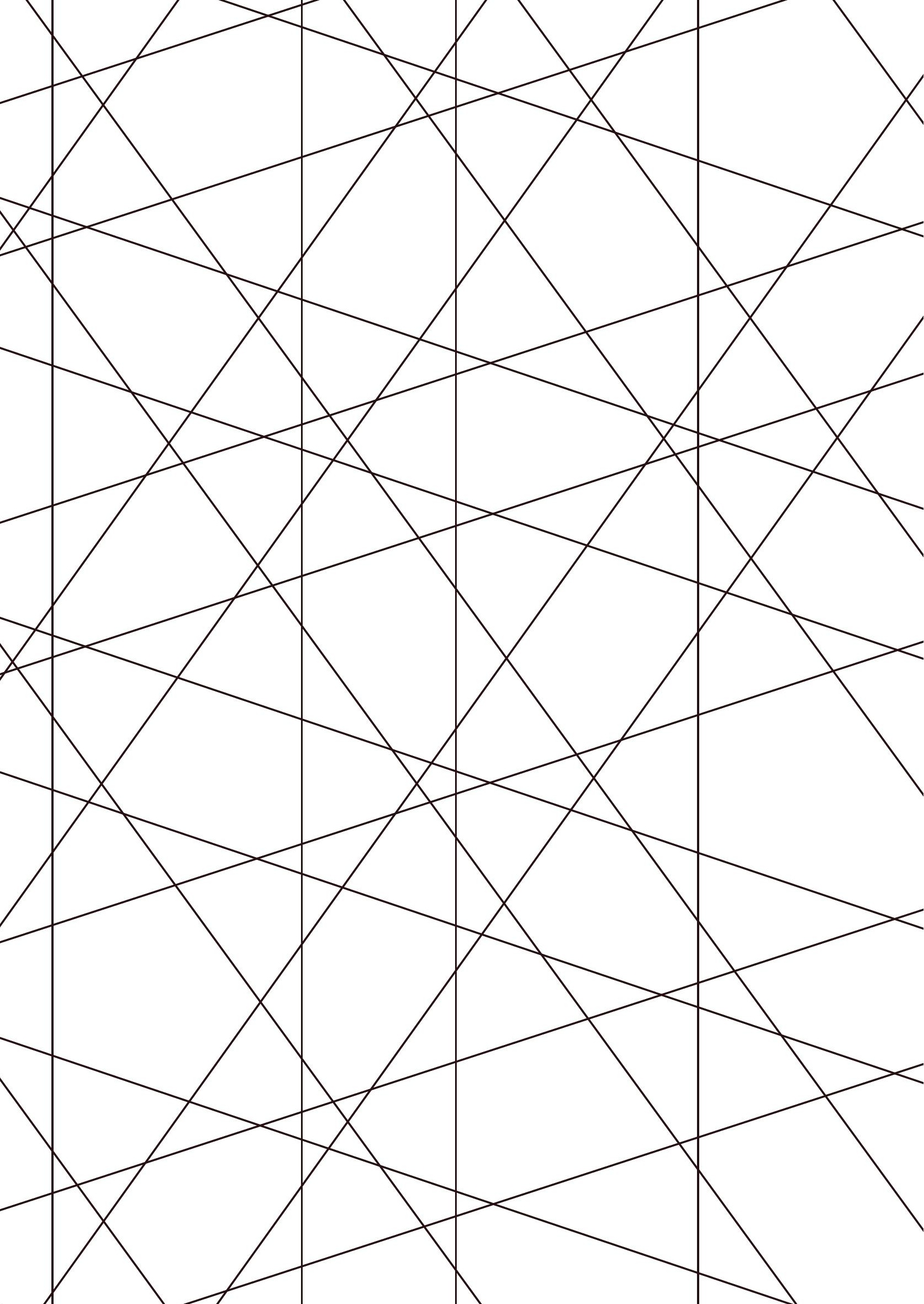




\section{CHAPTER 6 \\ Incidental findings on ${ }^{18} \mathrm{~F}$-fluorocholine PET/CT for parathyroid imaging}

Wouter A.M. Broos

Remco J.J. Knol

Friso M. van der Zant

Nicolaas C. Schaper

Maurits Wondergem

Submitted 


\section{Abstract}

Background: ${ }^{18} \mathrm{~F}$-fluorocholine $\left({ }^{18} \mathrm{~F}-\mathrm{FCH}\right) \mathrm{PET} / \mathrm{CT}$ is an upcoming imaging technique for localization of hyperfunctioning parathyroid glands. However, ${ }^{18} \mathrm{~F}-\mathrm{FCH}$ is a nonspecific tracer that also accumulates in malignancies, inflammatory lesions and several other benign abnormalities. The aim of this study was to determine the occurrence and relevance of incidental findings on ${ }^{18} \mathrm{~F}-\mathrm{FCH} \mathrm{PET} / \mathrm{CT}$ for parathyroid localization.

Methods: ${ }^{18} \mathrm{~F}-\mathrm{FCH}$ PET/CTs performed in our center for parathyroid localization from 2015 to 2019 were reviewed. Abnormal uptake of ${ }^{18} \mathrm{~F}-\mathrm{FCH}$, with or without anatomical substrate on the co-registered low-dose CT, and also incidental findings on CT without increased ${ }^{18} \mathrm{~F}-\mathrm{FCH}$ uptake were recorded. Each finding was correlated with follow-up data from the electronic medical records.

Results: A total of $388{ }^{18} \mathrm{~F}-\mathrm{FCH}$ PET/CTs were reviewed, with 247 incidental findings detected in 226 patients (58\%); $82{ }^{18} \mathrm{~F}-\mathrm{FCH}$ positive findings with corresponding pathology on CT, 16 without CT substrate and $149{ }^{18} \mathrm{~F}-\mathrm{FCH}$ negative abnormalities on CT. Malignant lesions were detected in 10/388 patients (2.6\%). Of all 98 detected ${ }^{18} \mathrm{~F}-\mathrm{FCH}$ positive lesions, 15 were malignant (15.3\%), concerning 4 metastases and 11 primary malignancies; breast carcinoma $(n=7)$, lung carcinoma $(n=2)$, thyroid carcinoma $(n=1)$ and skin melanoma $(n=1)$.

Conclusion: Clinically relevant incidental findings were observed in a substantial number of patients. In $15.3 \%$ of the incidental ${ }^{18} \mathrm{~F}-\mathrm{FCH}$ positive findings, the lesions were malignant. These data contribute to better knowledge of ${ }^{18} \mathrm{~F}-\mathrm{FCH}$ distribution, enhance interpretation of ${ }^{18} \mathrm{~F}-\mathrm{FCH}$ PET/CT and guide follow-up of incidental findings. Attention should especially be paid to breast lesions in this particular patient group with hyperparathyroidism in which women are typically over-represented. 


\section{Introduction}

In recent years, ${ }^{18} \mathrm{~F}$-fluorocholine $\left({ }^{18} \mathrm{~F}-\mathrm{FCH}\right)$ PET/CT has been established as a valuable imaging modality for preoperative identification of hyperfunctioning parathyroid glands in patients with hyperparathyroidism, which is a prerequisite for planning of minimally invasive parathyroidectomy.[1,2] Choline is a precursor for the biosynthesis of phosphatidylcholine, an essential component of the cell membrane. After uptake of choline by the cell, it is phosphorylated by the enzyme choline kinase and is retained in the cell. Cells with a high proliferation rate have increased demand for choline due to increased cell wall membrane synthesis which is mainly regulated by increased activity of choline kinase. After radiolabeling choline with a positron emitter such as ${ }^{18} \mathrm{~F}$, it can be used as a PET tracer to visualize tissue with high phospholipogenesis.[3] ${ }^{18} \mathrm{~F}-\mathrm{FCH}$ PET/CT has been proven beneficial in detecting certain types of malignancy, especially prostate cancer, and has also been investigated in several other malignancies such as hepatocellular carcinoma, brain tumors, lung tumors, breast cancer and genitourinary tumors.[4] However, ${ }^{18} \mathrm{~F}-\mathrm{FCH}$ uptake may also be observed in benign conditions such as parathyroid adenomas, as was initially noticed in a patient scanned for prostate cancer. [5]

Knowledge about various causes of ${ }^{18} \mathrm{~F}-\mathrm{FCH}$ uptake is of major importance for accurate scan interpretation and to recommend appropriate follow-up. Previously published data on ${ }^{18} \mathrm{~F}-\mathrm{FCH} \mathrm{PET} / \mathrm{CT}$ incidental findings were retrieved from cohorts of patients with prostate cancer.[6,7] However, ${ }^{18} \mathrm{~F}-\mathrm{FCH}$ PET/CT for hyperparathyroidism involves a different population in which, for example, women are over-represented. Also, the part of the body that is imaged is different. Therefore, it is expected that both the type and distribution of incidental findings on these scans is different from the abovementioned reports. In the present study, a large cohort of patients with hyperparathyroidism who received ${ }^{18} \mathrm{~F}-\mathrm{FCH} \mathrm{PET} / \mathrm{CT}$ was retrospectively analyzed to assess the frequency and relevance of incidental findings on ${ }^{18} \mathrm{~F}-\mathrm{FCH} \mathrm{PET} / \mathrm{CT}$. 


\section{Methods}

Reports of all ${ }^{18} \mathrm{~F}-\mathrm{FCH}$ PET/CT scans performed for hyperfunctioning parathyroid gland localization between June 2015 and September 2019 were reviewed. All incidental findings on PET or CT were recorded into a database. These findings were correlated with follow-up imaging, histopathological examinations and clinical follow-up data, retrieved from the electronic patient records. The follow-up period was at least six months. Incidental findings were categorized following anatomical localization: thyroid, lung, mediastinum, lymph nodes, breast, upper abdomen, skeleton and skin. Normal variants, vascular calcifications and degenerative bone changes on CT were excluded from further analysis. In patients who received more than one ${ }^{18} \mathrm{~F}-\mathrm{FCH} \mathrm{PET} / \mathrm{CT}$, only the first scan showing additional findings was included; subsequent scans were used as follow-up data.

\section{Informed consent}

All patients gave written informed consent for use of their anonymized data for scientific purposes. Besides the standard imaging protocol and clinical management no additional measurements or actions affecting the patient were performed. The study was approved by the institutional research department and performed in accordance with the Declaration of Helsinki. Approval of the local ethical committee for the present study was not necessary since the study does not fall within the scope of the Dutch Medical Research Involving Human Subjects Act (section 1.b WMO, 26th February 1998).

\section{Scan acquisition}

PET/CT images were acquired on a Siemens Biograph-16 TruePoint PET/CT camera (Siemens Healthcare). Dual-time-point images were acquired at $5 \mathrm{~min}$ and $60 \mathrm{~min}$ after intravenous injection of approximately $150 \mathrm{MBq}{ }^{18} \mathrm{~F}-\mathrm{FCH}$, ranging from the temporomandibular joint to the diaphragm. Images were acquired at $480 \mathrm{~s}$ per bed position with matrix size $256 \times 256$ and low-dose CT for attenuation correction using a tube current of 40-80 mAs at 100-120 kV with CARE Dose 4D dose modulation, collimation of $24 \times 1.2 \mathrm{~mm}$ and a pitch of 0.95 . PET images were reconstructed with an iterative 3D method using 5 iterations, 8 subsets and a Gaussian filter. Patient preparation consisted of hydration with $1 \mathrm{~L}$ water and, if applicable, discontinuation of colchicine $48 \mathrm{~h}$ prior to ${ }^{18} \mathrm{~F}-\mathrm{FCH}$ administration. Discontinuation of calcimimetic drugs or other medications was not required. 


\section{Statistical analysis}

Normally distributed continuous data were expressed as mean \pm standard deviation and range. Non-continuous data were expressed as numbers with percentages. The occurrences of incidental findings were calculated as percentages of the whole cohort. The analysis was carried out using the Statistical Package for Social Sciences 25 (IBM SPSS Statistics, Chicago, IL, USA).

\section{Results}

A total of $408{ }^{18} \mathrm{~F}-\mathrm{FCH}$ PET/CT scans were performed between June 2015 and September 2019. Twenty were excluded as these were follow-up scans of already included ${ }^{18} \mathrm{~F}-\mathrm{FCH}$ PET/CT scans. The reports of the other 388 scans were reviewed for incidental findings. Patient characteristics of this cohort are listed in Table 1. The mean follow-up period was 31 months (range 6-57). A total of 247 incidental findings were observed in 226 patients (58\%) (Table 2). There were $82{ }^{18} \mathrm{~F}-\mathrm{FCH}$ positive findings with accompanying abnormalities on $\mathrm{CT}$ in 70 patients (18\%), $16{ }^{18} \mathrm{~F}-\mathrm{FCH}$ positive findings without evidence of pathology on CT in 15 patients (4\%), and 149 CT abnormalities without pathological ${ }^{18} \mathrm{~F}$-FCH uptake in 130 patients (34\%). Of all $98{ }^{18} \mathrm{~F}-\mathrm{FCH}$ positive incidental findings, 15 were malignant (15.3\%) concerning 11 primary malignancies and 4 metastases. These malignant lesions were detected in 10 patients (2.6\%). None of the ${ }^{18} \mathrm{~F}-\mathrm{FCH}$ negative CTfindings were proven to be malignant during follow-up.

Table 1. Patient characteristics

\begin{tabular}{lc}
\hline Age [mean \pm SD (range)] (years) & $62 \pm 12(25-86)$ \\
Sex [n (\%)] & $98(25)$ \\
Male & $290(75)$ \\
$\quad$ Female & 354 \\
Type of hyperparathyroidism [n] & 15 \\
$\quad$ Primary & 13 \\
$\quad$ Secondary & 6 \\
$\quad$ Tertiary & $20.3 \pm 29.7(1.9-339.9)$ \\
$\quad$ Unclear & $2.64 \pm 0.21(2.01-3.86)$ \\
PTH [mean \pm SD (range)] (pmol/L) & \\
$\quad$ (normal range: $1.3-6.8$ pmol/L) & \\
Serum calcium [mean \pm SD (range)] (mmol/L) & \\
$\quad$ (normal range: $2.10-2.50$ mmol/L) &
\end{tabular}


Table 2. Number of incidental findings detected on ${ }^{18} \mathrm{~F}$-fluorocholine PET/CT scans for hyperparathyroidism categorized by anatomical localization.

\begin{tabular}{lcccc}
\hline Category & \multicolumn{2}{c}{ Incidental findings } & $\begin{array}{c}\text { Primary } \\
\text { malignancies* }\end{array}$ & Metastases* \\
\cline { 2 - 3 } Thyroid & Choline-avid & Not choline-avid & 1 & 1 \\
Lung & 18 & 42 & 2 & 1 \\
Mediastinum & 26 & 21 & 0 & 0 \\
Lymph nodes & 6 & 25 & 0 & 1 \\
Breast & 6 & 2 & 7 & 0 \\
Upper abdomen & 10 & 4 & 0 & 0 \\
Skeleton & 7 & 47 & 0 & 1 \\
Skin & 22 & 7 & 1 & 0 \\
Total & 3 & 1 & $\mathbf{1 1}$ & $\mathbf{4}$ \\
\hline
\end{tabular}

* all detected primary malignancies and metastases were ${ }^{18} \mathrm{~F}$-fluorocholine avid

\section{Thyroid}

In 20 patients a solitary thyroid nodule was detected, of which 10 nodules showed increased ${ }^{18} \mathrm{~F}-\mathrm{FCH}$ uptake. Histology was acquired from six choline-avid lesions, showing one papillary thyroid carcinoma (Fig. 1), one metastasis of renal cell carcinoma and four benign etiologies (colloid cysts and hyperplastic nodules). Two of the cholineavid nodules and five of the nodules without uptake were not suspicious on follow-up ultrasonography. No adequate follow-up data were available for the other nodules. Multinodular goiter with irregular ${ }^{18} \mathrm{~F}-\mathrm{FCH}$ uptake was described in 35 patients and a diffusely increased uptake was seen in five patients ( 2 hyperthyroid patients, 2 hypothyroid patients and 1 patient with normal thyroid function).

\section{Lung}

Increased ${ }^{18} \mathrm{~F}-\mathrm{FCH}$ in the lungs was observed in 26 patients; in 2 of these patients no CT substrate was visible and no follow-up was available; in 18 of these patients the coregistered CT and follow-up imaging revealed infectious infiltrates and in 6 of the 26 patients this concerned uptake in nodular lesions (mean diameter of $10 \mathrm{~mm}$, range 6-19 $\mathrm{mm}$ ). Of these, one was confirmed as primary (squamous) lung carcinoma following resection (Fig. 2), one was regarded as lung carcinoma based on imaging and treated by external radiation therapy and one was regarded as pulmonary metastasis in a patient with known metastasized renal cell carcinoma. The other 3 patients were, according to follow-up imaging with CT or ${ }^{18} \mathrm{~F}$-fluorodesoxyglucose (18 F-FDG) PET/CT, diagnosed with inflammatory lung disease. Lung nodules without ${ }^{18} \mathrm{~F}-\mathrm{FCH}$ uptake were detected in 21 patients (mean diameter of $8 \mathrm{~mm}$, range $6-13 \mathrm{~mm}$ ). None were suspicious for malignancy on follow-up imaging with CT ( $n=13),{ }^{18 F-F D G ~ P E T / C T ~}(n=2)$ or chest X-ray ( $\left.n=3\right)$, no followup was available for the other three patients. 


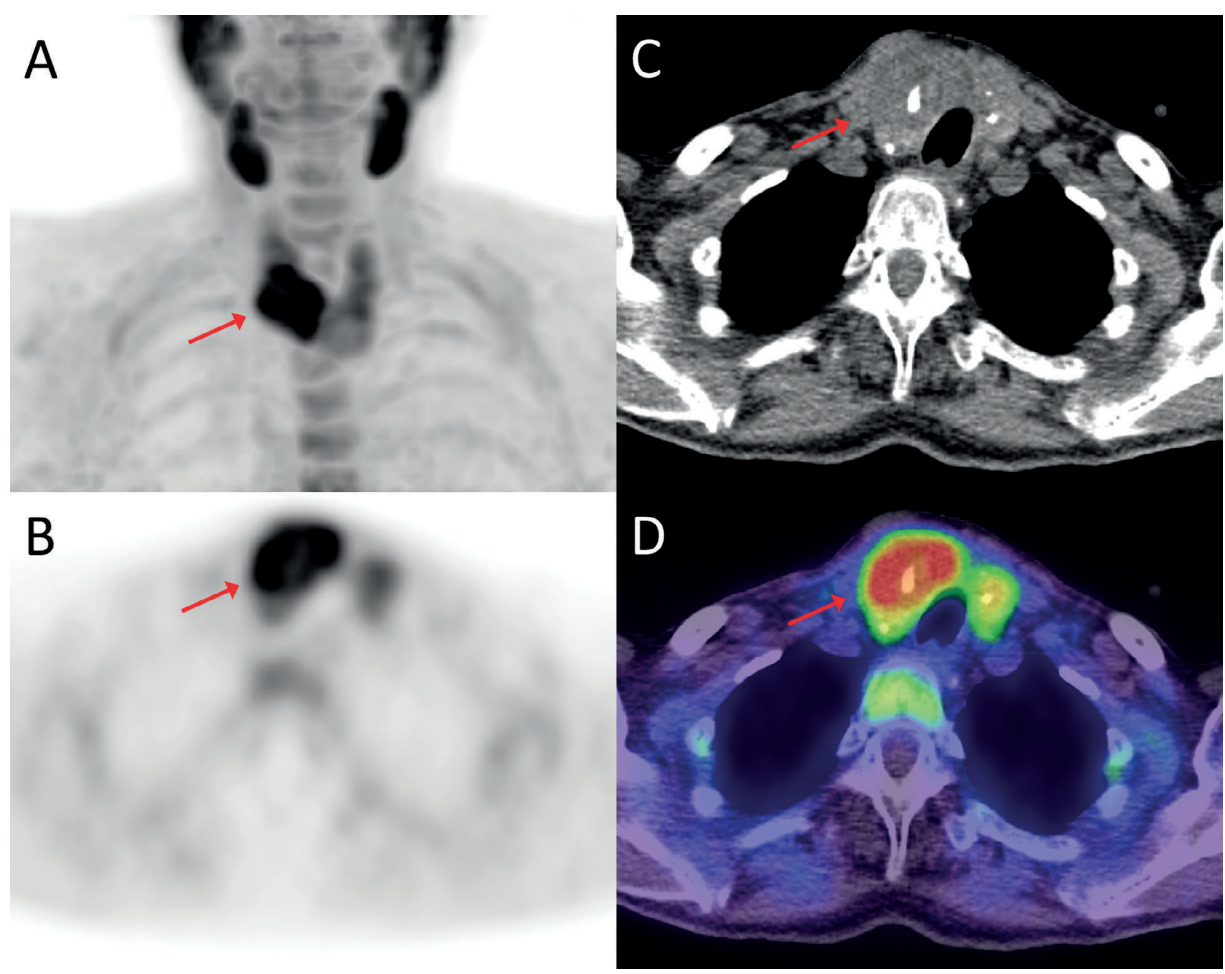

Figure 1. Maximum-intensity projection of ${ }^{18} \mathrm{~F}$-fluorocholine $\left({ }^{18} \mathrm{~F}-\mathrm{FCH}\right)$ PET (A) and transaxial views of the thyroid gland on PET (B), CT (C) and PET/CT fusion images (D) showing intense ${ }^{18} \mathrm{~F}-\mathrm{FCH}$ uptake in the enlarged right thyroid lobe (SUV $\left.{ }_{\max } 8.2\right)$. Histopathologic examination revealed a pT3a papillary thyroid carcinoma.
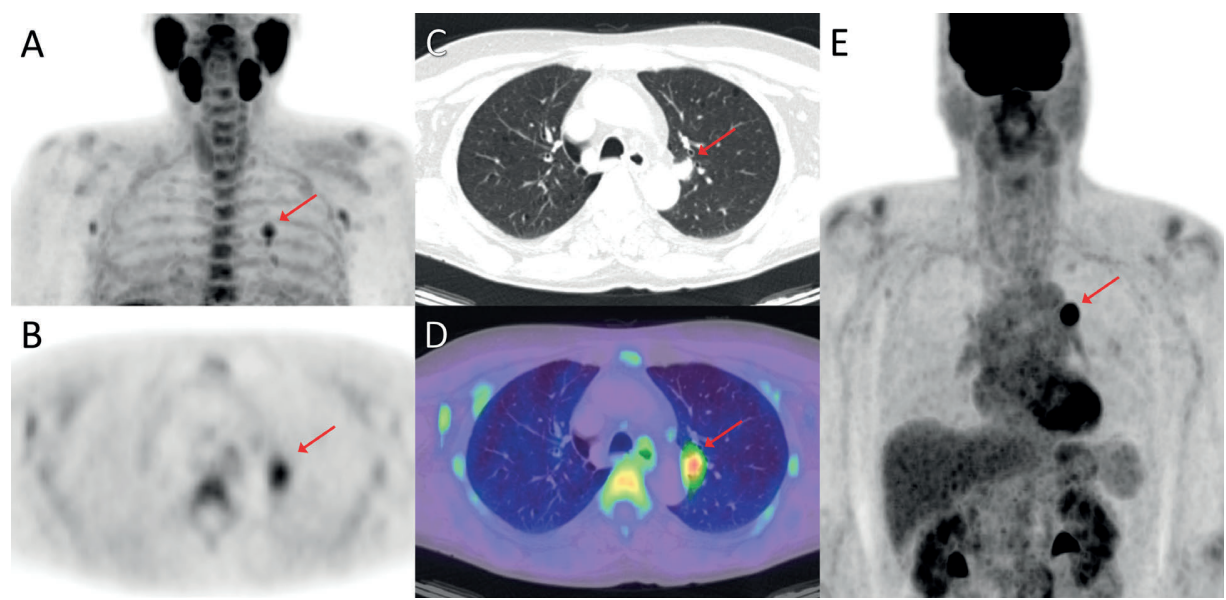

Figure 2. Maximum-intensity projection (MIP) of ${ }^{18} \mathrm{~F}$-fluorocholine $\left({ }^{18} \mathrm{~F}-\mathrm{FCH}\right) \mathrm{PET}(\mathbf{A})$ and transaxial views of the lungs on PET (B), CT (C) and PET/CT fusion images (D) showing focal ${ }^{18} \mathrm{~F}-\mathrm{FCH}$ uptake in a left upper lobe lung nodule (diameter $1.8 \mathrm{~cm}$, SUV max $_{\text {m }} 3.6$ ). MIP of ${ }^{18}$ F-FDG PET (E) showing intense FDG-avidity (SUV ${ }_{\text {max }}$ 9.2) without evidence for metastases. Histopathological examination after lobectomy revealed pT1b squamous cell lung carcinoma. 


\section{Mediastinum}

Increased ${ }^{18} \mathrm{~F}-\mathrm{FCH}$ uptake in lesions in the anterior mediastinum was detected in four patients. Three of those were under $30 \mathrm{y}$ of age and since follow-up imaging with CT or MRI showed involution of the lesions, the uptake was regarded as physiological uptake in residual thymus gland. In the other, 50-year-old, patient the lesion was not suspicious for malignancy on a follow-up ${ }^{18} \mathrm{~F}$-FDG PET/CT but no certain diagnosis was established. Increased ${ }^{18} \mathrm{~F}-\mathrm{FCH}$ uptake in the esophagus was seen in two patients, one due to a candida infection and one to inflammation associated with bulimia nervosa. The esophagus of one patient showed achalasia on CT and an esophageal hiatus hernia was found in 24 patients.

\section{Lymph nodes}

In six patients, increased ${ }^{18} \mathrm{~F}-\mathrm{FCH}$ uptake was detected in enlarged lymph nodes $(>1 \mathrm{~cm})$. Another two patients presented with enlarged lymph nodes without ${ }^{18} \mathrm{~F}-\mathrm{FCH}$ uptake. In one of the patients with choline-avid enlarged lymph nodes, breast cancer metastasis was histologically proven. No metastases, malignant lymphomas or granulomatous lymphadenopathies were diagnosed in the other patients during follow-up with CT $(n=4)$, ultrasonography $(n=2)$ or histopathological evaluation $(n=1)$.

\section{Breast}

Focally increased ${ }^{18} \mathrm{~F}$-FCH uptake in the breasts was detected in 10 patients (Fig. 3). Breast malignancy was histologically proven in seven patients (3 patients with invasive ductal carcinoma, 2 patients with invasive lobular carcinoma, 1 patient with invasive carcinoma of no special type and 1 patient with ductal carcinoma in situ in the left breast and mucinous carcinoma in the right breast). Follow-up imaging with mammography or ultrasonography in the other three patients did not reveal suspicious lesions and no histopathology was acquired. CT abnormalities without ${ }^{18} \mathrm{~F}-\mathrm{FCH}$ uptake were detected in four patients (benign fibrosis in one patient and fibro-adenomas in three patients).

\section{Upper abdomen}

In six patients, increased uptake of ${ }^{18} \mathrm{~F}-\mathrm{FCH}$ was observed in adrenal adenomas with a typical benign appearance on CT (Fig. 4). Diffusely increased ${ }^{18} \mathrm{~F}$-FCH uptake was observed in a cirrhotic liver in one patient. Furthermore, multiple ${ }^{18} \mathrm{~F}-\mathrm{FCH}$ negative findings were identified on CT, such as benign liver lesions ( $n=28$, e.g.: cysts, hemangiomas), splenic cyst $(n=1)$, adrenal myelolipoma $(n=1)$, benign kidney abnormalities ( $n=6$, e.g.: cysts, cortical atrophy) and gallstones ( $n=11)$. 


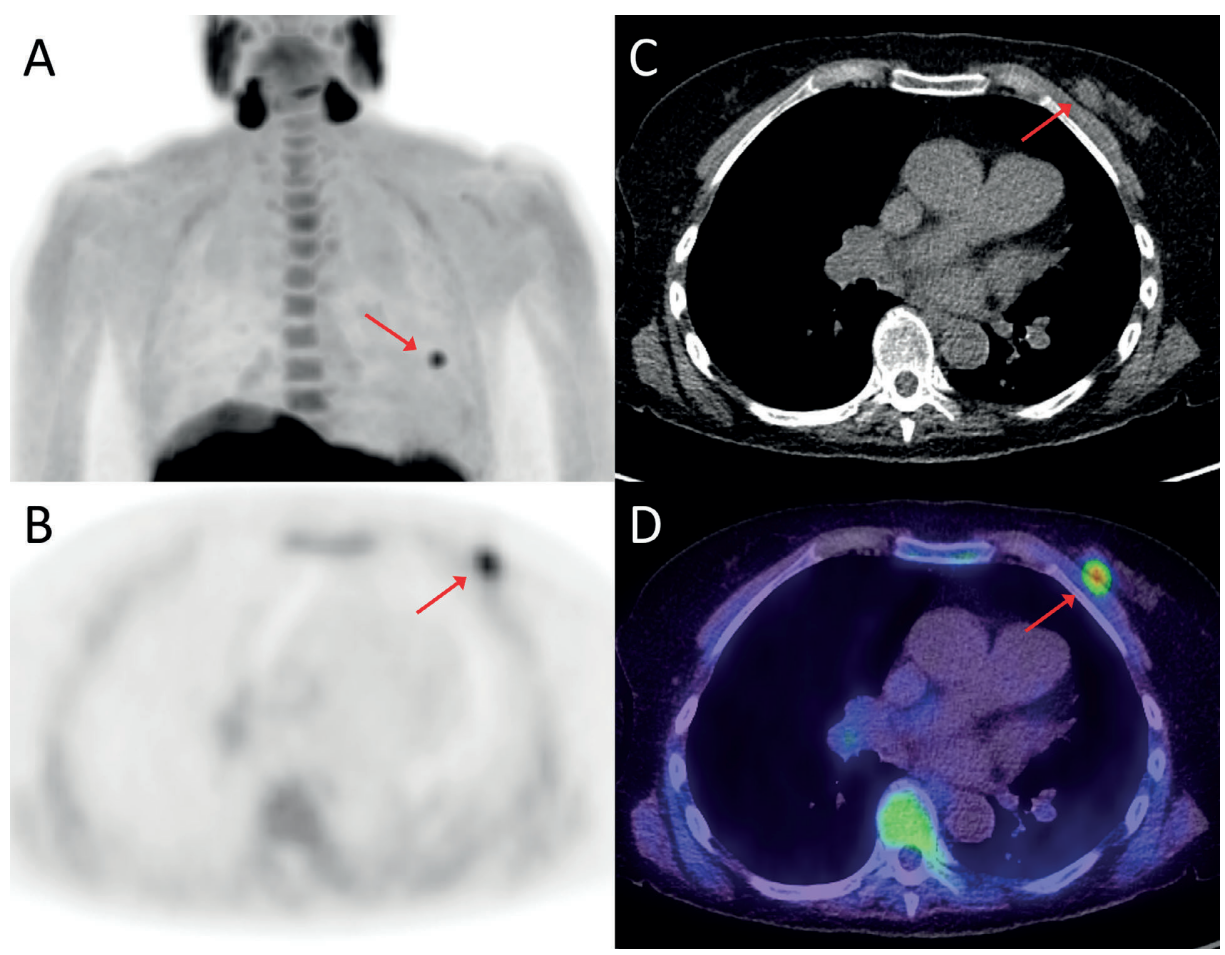

Figure 3. Maximum-intensity projection of ${ }^{18} \mathrm{~F}$-fluorocholine $\left({ }^{18} \mathrm{~F}-\mathrm{FCH}\right) \mathrm{PET}(\mathbf{A})$ and transaxial views of the breasts on PET (B), CT (C) and PET/CT fusion images (D) showing focal ${ }^{18} \mathrm{~F}-\mathrm{FCH}$ uptake in a nodular mass in the left breast (diameter $1.6 \mathrm{~cm}$, SUV $_{\max } 4.5$ ). Histopathologic examination revealed a pT1c infiltrating lobular breast carcinoma.
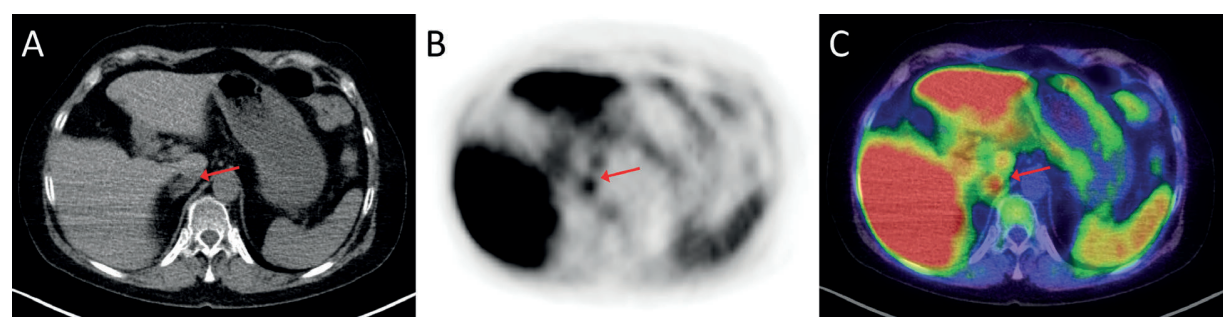

Figure 4. Transaxial views of the upper abdomen on CT (A), PET (B) and PET/CT fusion images (C) showing focal ${ }^{18} \mathrm{~F}$-fluorocholine uptake in a nodular mass of the right adrenal gland (SUV $\left.{ }_{\max } 6.6\right)$ and a typical aspect of an adrenal adenoma on CT (radiodensity of -5 Hounsfield units).

\section{Skeleton}

A physiological, diffuse uptake of ${ }^{18} \mathrm{~F}-\mathrm{FCH}$ was regularly seen in the bone marrow. In 12 patients, fractures were detected of which 8 showed an increased uptake of ${ }^{18} \mathrm{~F}-\mathrm{FCH}$ (mainly rib fractures and vertebral compression fractures). Choline-avid degenerative changes were observed in four patients, a choline-avid osteoid osteoma in one patient and vertebral hemangiomas with decreased ${ }^{18} \mathrm{~F}-\mathrm{FCH}$ uptake in three patients. In two 
patients, both diagnosed with tertiary hyperparathyroidism, a diffusely increased uptake of ${ }^{18} \mathrm{~F}$-FCH was noticed in the skeleton, accompanied with diffuse sclerosis on CT (reported earlier as case report [8]). Intensely increased ${ }^{18} \mathrm{~F}-\mathrm{FCH}$ uptake was detected in seven

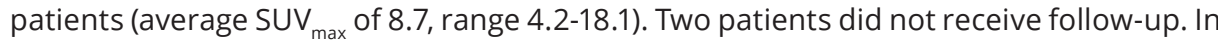
one patient the uptake was related to metastases of breast carcinoma. In three patients follow-up magnetic resonance imaging (MRI) did only show degenerative changes but no signs of malignancy. In the last patient, the lesions were negative on ${ }^{18} \mathrm{~F}-\mathrm{FDG}$ PET/CT and follow-up ${ }^{18} \mathrm{~F}-\mathrm{FCH}$ PET/CT showed evident decrease of uptake without any intervention (Fig. 5). No certain cause for the increased ${ }^{18} \mathrm{~F}-\mathrm{FCH}$ uptake could be established.

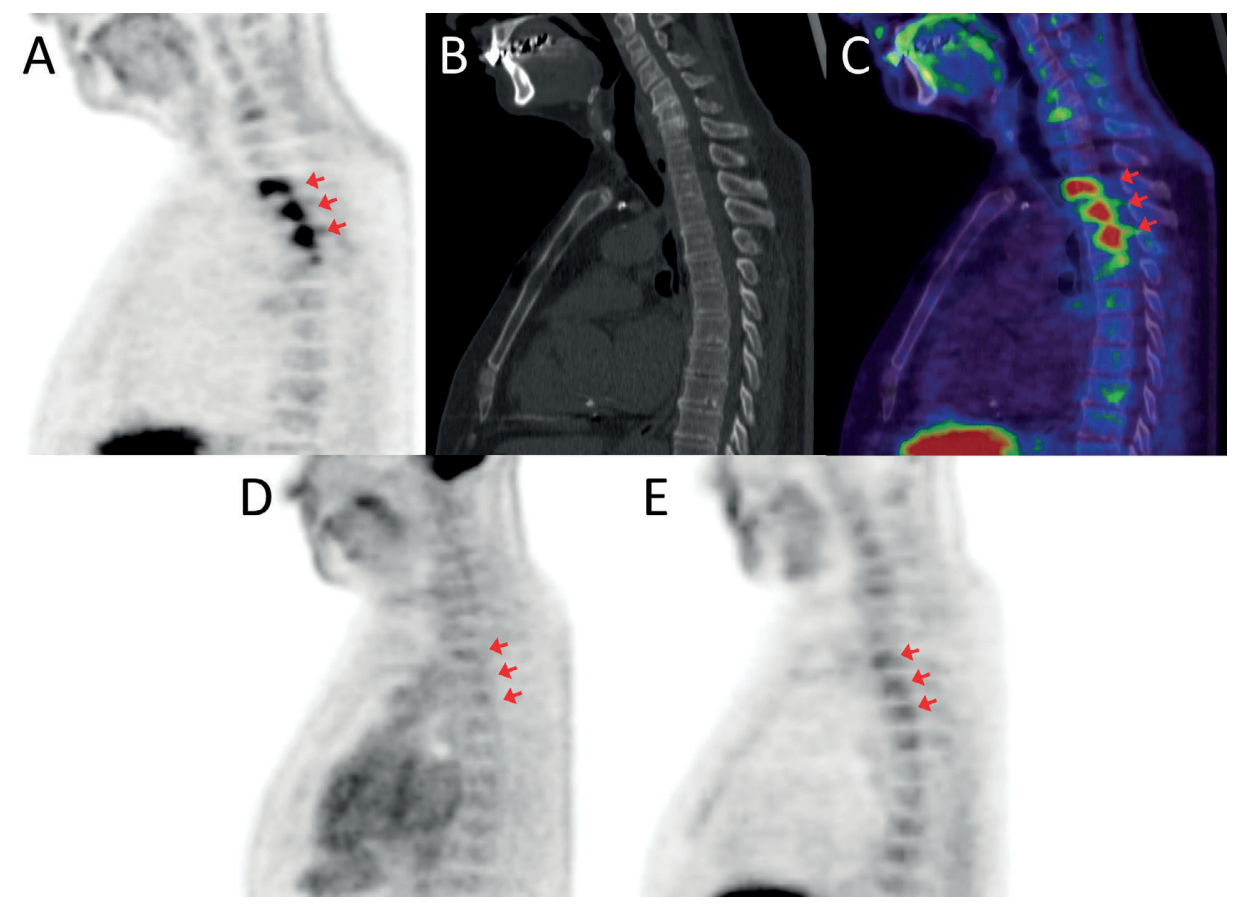

Figure 5. Sagittal views of ${ }^{18} \mathrm{~F}$-fluorocholine $\left({ }^{18} \mathrm{~F}-\mathrm{FCH}\right) \mathrm{PET}(\mathbf{A}), \mathrm{CT}(\mathbf{B})$ and PET/CT fusion images (C) showing an example of nonspecific intense bone uptake (thoracic vertebrae 2-4, SUV ${ }_{\max }$ 18.1). Additional ${ }^{18}$ F-FDG PET (D) did not show signs of malignancy and follow-up ${ }^{18} \mathrm{~F}-\mathrm{FCH}$ PET (E) 3 months later showed a near normalization of uptake. The cause of the increased ${ }^{18} \mathrm{~F}-\mathrm{FCH}$ uptake was not elucidated.

\section{Skin}

Two focally choline-avid cutaneous lesions were detected. One lesion proved to be melanoma upon histopathological evaluation, published earlier in a case report.[9] No follow-up of the other lesion was available. More diffusely increased cutaneous ${ }^{18} \mathrm{~F}-\mathrm{FCH}$ uptake was observed in a patient with erysipelas and multiple subcutaneous lesions without ${ }^{18} \mathrm{~F}-\mathrm{FCH}$ uptake in another patient resembled sebaceous cysts. 


\section{Discussion}

In the past decades, ${ }^{18} \mathrm{~F}-\mathrm{FCH}$ has been extensively used in $\mathrm{PET} / \mathrm{CT}$ imaging for prostate cancer as a valuable alternative to ${ }^{18} \mathrm{~F}-\mathrm{FDG}$, due to its capability to accumulate in lesions with a low rate of glucose metabolism. However, choline is also known to accumulate in various other malignancies and has been investigated for several tumors, with variable results.[4] The reported variety of secondary malignancies detected on choline PET/ CT for prostate cancer imaging is extensive and comprises malignancies of the thyroid, lung, esophagus, colon, kidney and bladder, as well as melanoma, lymphoma, glioma, multiple myeloma, pleural mesothelioma and invasive thymoma.[7,10-12] In larger prostate cancer patient cohorts, secondary malignancies were discovered on choline PET/CT in 10/1000 (1\%) patients by Calabria et al., in 7/454 (1.5\%) patients by Garcia et al. and in $2 / 77(2.6 \%)$ by How Kit et al.[7,10,13] A study specifically focussed on incidental ${ }^{18} \mathrm{~F}-\mathrm{FCH}$ uptake in the thyroid gland found 2 malignant thyroid lesions in a cohort of 368 patients (0.5\%).[14]

In the present study, ${ }^{18} \mathrm{~F}-\mathrm{FCH}$ PET/CT scans were analyzed that were performed for localization of hyperfunctioning parathyroid glands in patients with hyperparathyroidism. Of all $98{ }^{18} \mathrm{~F}-\mathrm{FCH}$ positive incidental findings, 15 were malignant (15.3\%), newly detected in $10 / 388$ patients (2.6\%). The most frequently found malignancy was breast cancer, which was detected in 7 patients. In two of those breast cancer patients a coincidental second primary tumor was detected (one thyroid carcinoma and one skin melanoma) and in another breast cancer patient metastases to the bone and lymph nodes were detected. Lung cancer was diagnosed in two patients and renal cell carcinoma metastases to the lung and thyroid gland in another patient. Several case reports of incidental findings on ${ }^{18} \mathrm{~F}-\mathrm{FCH}$ PET/CT for hyperparathyroidism are available; however, to our knowledge, no comparable patient cohort has been studied. The population scanned for hyperparathyroidism often consists of younger patients and relatively more women are represented, as a result of a female-to-male prevalence ratio of approximately 2.5 to 1.[15] In the current study this ratio was 3 to 1, which explains the relatively high number of incidentally found breast malignancies. Distribution and pitfalls of ${ }^{18} \mathrm{~F}-\mathrm{FCH}$ in female patients was studied in a small cohort of 21 breast cancer patients, but besides uptake in breast cancer and metastases, only benign incidental findings were found.[16] Another difference with earlier studied prostate cancer cohorts is the scan range, which for parathyroid PET/CT typically is limited to the level of the diaphragm. One research group purposely scanned to the level of the pelvis in men and the liver in women, in order not to miss prostate, breast or hepatocellular carcinoma, but in their small cohort, none of these were detected.[17,18] Furthermore, the risk of a second primary malignancy in 
cancer patients is higher than the risk of cancer among the general population.[19] In contrast, the present study concerns PET/CT performed for benign pathology.

Apart from ${ }^{18} \mathrm{~F}-\mathrm{FCH}$ uptake in malignancies and physiologic uptake in liver, spleen, pancreas, kidney, bone marrow and salivary glands, ${ }^{18} \mathrm{~F}-\mathrm{FCH}$ uptake can also be observed in several benign conditions.[6] In the aforementioned study by Calabria et al., ${ }^{18} \mathrm{~F}-\mathrm{FCH}$ uptake was detected in inflammation related findings in 80/1000 patients (e.g. lymph nodes, skin, thyroid, lungs), benign tumour uptake in 26/1000 patients (e.g. meningiomas, colon adenomas, thymomas), uptake associated with hypermetabolism in 7/1000 patients (e.g. hyperthyroidism, adrenal adenomas) and nonspecific uptake in 46/1000 patients (abnormal uptake without clinical evidence, lab tests or correlative imaging). [7] In a retrospective study on ${ }^{11} \mathrm{C}$-choline PET/CT in 2933 men with prostate cancer, parathyroid adenoma was diagnosed in 13 patients.[20] In the present study, incidental ${ }^{18} \mathrm{~F}-\mathrm{FCH}$ uptake with a high likelihood of benign aetiology was seen in 78/388 patients (20\%) and the most common cause of increased ${ }^{18} \mathrm{~F}-\mathrm{FCH}$ uptake was inflammation. In inflammatory tissue ${ }^{18} \mathrm{~F}-\mathrm{FCH}$ is mainly accumulated in macrophages, as was demonstrated in mice by Wyss et al.[21], to increase phosphatidylcholine biosynthesis which primes the macrophages to respond appropriately to immune stimuli.[22]

Several patients in the studied cohort showed intense osseous uptake of ${ }^{18} \mathrm{~F}-\mathrm{FCH}$ without a clear explanation despite thorough follow-up. Since literature also provides no clues regarding the aetiology of those lesions, these are considered nonspecific. Degenerative changes have been shown to accumulate ${ }^{18} \mathrm{~F}-\mathrm{FCH}$, probably due to inflammation.[23] Moreover, we hypothesize that occult (micro) fractures could lead to increased ${ }^{18} \mathrm{~F}-\mathrm{FCH}$ uptake. Also, some benign bone lesions have been shown to demonstrate uptake, such as fibrous dysplasia.[24] Besides known uptake in bone metastases from prostate carcinoma and other malignancies, uptake of ${ }^{18} \mathrm{~F}-\mathrm{FCH}$ has also been described in multiple myeloma and various primary bone tumours.[4,25,26]

A total of 154 findings without increased ${ }^{18} \mathrm{~F}-\mathrm{FCH}$ uptake were detected on the coregistered low-dose CT in 134 patients (35\%). In the literature, a variable frequency of incidental findings on low-dose CT has been reported for PET/CT with other radiopharmaceuticals, such as ${ }^{18} \mathrm{~F}-\mathrm{FDG},{ }^{18} \mathrm{~F}$-sodium fluoride and ${ }^{13} \mathrm{~N}$-ammonia, ranging from $51-93 \%$, and with potentially clinical significance in $8-23 \%$ of patients.[27-29] In case of incidentally detected abnormalities on the co-registered low-dose CT, it is advised to follow radiological guidelines such as recommendations of the Fleischner Society or the American College of Radiology incidental findings committee.[30,31] 
Limitations of this study are its retrospective design and the relatively short follow-up period for some patients. Also, in several patients with additional findings no followup imaging or histopathological examination was available, therefore the number of detected malignancies may be underestimated. One lung carcinoma and one pulmonary metastasis were not biopsied but treatment was initiated based on the convincing imaging results only, all other reported malignancies were histopathologically proven.

To summarize, increased ${ }^{18} \mathrm{~F}-\mathrm{FCH}$ uptake during PET/CT parathyroid imaging resulted in a variety of incidental findings in $58 \%$ of the patients and malignancies were detected in $2.6 \%$. The chance of an incidental ${ }^{18} \mathrm{~F}-\mathrm{FCH}$ positive finding to be malignant was $15.3 \%$. In general, we advise additional imaging or biopsy for all focal ${ }^{18} \mathrm{~F}-\mathrm{FCH}$ uptake in the breasts or thyroid gland. Also, focal pulmonary uptake should warrant biopsy or followup imaging. Cutaneous uptake should be correlated with at least visual examination of the skin. Moderate ${ }^{18} \mathrm{~F}-\mathrm{FCH}$ uptake in lymph nodes is frequently benign or physiological; however, no maximum uptake value can be given to discriminate pathological lymph nodes based on the results of this study. In any case, patients with enlarged lymph nodes or nodes with a typical pathologic distribution pattern should be further analysed. Focal uptake in the skeleton can be nonspecific and, in the absence of a corresponding benign lesion on CT, further analysis is advised to exclude malignancy. Non choline-avid CT abnormalities should be classified according to radiological guidelines.

\section{Conclusion}

In this study, a substantial number of incidental findings were observed in ${ }^{18} \mathrm{~F}-\mathrm{FCH} \mathrm{PET/CT}$ scans performed for parathyroid adenoma localization, including malignancies in $2.6 \%$ of the patients. Since a large number of patients scanned for hyperparathyroidism are women, relatively high numbers of breast cancer were incidentally detected. These data contribute to better knowledge of both physiological and pathological ${ }^{18} \mathrm{~F}-\mathrm{FCH}$ uptake in the human body and therefore enhance interpretation of ${ }^{18} \mathrm{~F}-\mathrm{FCH}$ PET/CT and guide follow-up of incidental findings. 


\section{References}

1. Thanseer N, Bhadada SK, Sood A, Mittal BR, Behera A, Gorla AKR, et al. Comparative effectiveness of

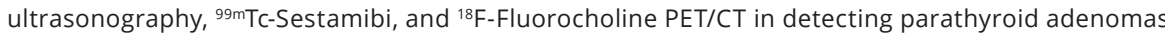
in patients with primary hyperparathyroidism. Clin Nucl Med 2017;42:e491-e497.

2. Cuderman A, Senica K, Rep S, Hocevar M, Kocjan T, Jensterle Sever M, et al. ${ }^{18} \mathrm{~F}$-Fluorocholine PET/ CT in primary hyperparathyroidism: superior diagnostic performance to conventional scintigraphic imaging for localization of hyperfunctioning parathyroid glands. J Nucl Med 2020;61:577-583.

3. Vallabhajosula S. ${ }^{18} \mathrm{~F}$-labeled positron emission tomographic radiopharmaceuticals in oncology: an overview of radiochemistry and mechanisms of tumor localization. Semin Nucl Med 2007;37:400-419.

4. Treglia G, Giovannini E, Di Franco D, Calcagni ML, Rufini V, Picchio M, et al. The role of positron emission tomography using carbon-11 and fluorine-18 choline in tumors other than prostate cancer: a systematic review. Ann Nucl Med 2012;26:451-461.

5. Quak E, Lheureux S, Reznik Y, Bardet S, Aide N. F18-choline, a novel PET tracer for parathyroid adenoma? J Clin Endocrinol Metab 2013;98:3111-3112.

6. Beheshti M, Haroon A, Bomanji JB, Langsteger W. Fluorocholine PET/computed tomography: physiologic uptake, benign findings, and pitfalls. PET Clin 2014;9:299-306.

7. Calabria F, Chiaravalloti A, Ciccio C, Gangemi V, Gulla D, Rocca F, et al. PET/CT with ${ }^{18}$ F-choline: physiological whole bio-distribution in male and female subjects and diagnostic pitfalls on 1000 prostate cancer patients: ${ }^{18} \mathrm{~F}$-choline PET/CT bio-distribution and pitfalls. A southern Italian experience. Nucl Med Biol 2017;51:40-54.

8. Broos WAM, Wondergem M, van der Zant FM, Knol RJJ. Tertiary hyperparathyroidism with renal osteodystrophy on ${ }^{18} \mathrm{~F}$-fluorocholine PET/CT. Clin Nucl Med 2018;43:766-768.

9. Burgers AMG, Wondergem M, van der Zant FM, Knol RJJ. Incidental detection of a melanoma by ${ }^{18} \mathrm{~F}$-fluorocholine PET/CT performed for evaluation of primary hyperparathyroidism. Clin Nucl Med 2018;43:265-266.

10. Garcia JR, Ponce A, Canales M, Ayuso J, Moragas M, Soler M. Detection of second tumors in ${ }^{11} \mathrm{C}$-choline PET/CT studies performed due to biochemical recurrence of prostate cancer. Rev Esp Med Nucl Imagen Mol 2014;33:28-31.

11. Sollini M, Pasqualetti F, Perri M, Coraggio G, Castellucci $P$, Roncali $M$, et al. Detection of a second malignancy in prostate cancer patients by using $\left[{ }^{18} \mathrm{~F}\right]$ Choline PET/CT: a case series. Cancer Imaging 2016;16:27.

12. Welle CL, Cullen EL, Peller PJ, Lowe VJ, Murphy RC, Johnson GB, et al. ${ }^{11} \mathrm{C}$-Choline PET/CT in recurrent prostate cancer and nonprostatic neoplastic processes. Radiographics 2016;36:279-292.

13. How Kit N, Dugue AE, Sevin E, Allouache N, Lesaunier F, Joly F, et al. Pairwise comparison of ${ }^{18} \mathrm{~F}-\mathrm{FDG}$ and ${ }^{18} \mathrm{~F}-\mathrm{FCH}$ PET/CT in prostate cancer patients with rising PSA and known or suspected second malignancy. Nucl Med Commun 2016;37:348-355.

14. Albano D, Durmo R, Bertagna F, Giubbini R. ${ }^{18} \mathrm{~F}$-choline PET/CT incidental thyroid uptake in patients studied for prostate cancer. Endocrine 2019;63:531-536.

15. Yeh MW, Ituarte PH, Zhou HC, Nishimoto S, Liu IL, Harari A, et al. Incidence and prevalence of primary hyperparathyroidism in a racially mixed population. J Clin Endocrinol Metab 2013;98:1122-1129.

16. Ahmad Saad FF, Zakaria MH, Appanna B. PET/CT analysis of 21 patients with breast cancer: physiological distribution of ${ }^{18} \mathrm{~F}$-choline and diagnostic pitfalls. J Int Med Res 2018;46:3138-3148. 
17. Michaud L, Burgess A, Huchet V, Lefevre M, Tassart M, Ohnona J, et al. Is ${ }^{18} \mathrm{~F}$-fluorocholine-positron emission tomography/computerized tomography a new imaging tool for detecting hyperfunctioning parathyroid glands in primary or secondary hyperparathyroidism? J Clin Endocrinol Metab 2014;99:45314536.

18. Michaud L, Balogova S, Burgess A, Ohnona J, Huchet V, Kerrou K, et al. A Pilot Comparison of 18F-fluorocholine PET/CT, Ultrasonography and ${ }^{123} /{ }^{99 m} \mathrm{mC}$-sestaMIBI dual-phase dual-isotope scintigraphy in the preoperative localization of hyperfunctioning parathyroid glands in primary or secondary hyperparathyroidism: influence of thyroid anomalies. Medicine (Baltimore) 2015;94:e1701.

19. Travis LB. The epidemiology of second primary cancers. Cancer Epidemiol Biomarkers Prev 2006;15:20202026.

20. Parvinian A, Martin-Macintosh EL, Goenka AH, Durski JM, Mullan BP, Kemp BJ, et al. ${ }^{11}$ C-Choline PET/ CT for detection and localization of parathyroid adenomas. AJR Am J Roentgenol 2017:1-5.

21. Wyss MT, Weber B, Honer M, Spath N, Ametamey SM, Westera G, et al. ${ }^{18} \mathrm{~F}$-choline in experimental soft tissue infection assessed with autoradiography and high-resolution PET. Eur J Nucl Med Mol Imaging 2004;31:312-316.

22. Snider SA, Margison KD, Ghorbani P, LeBlond ND, O'Dwyer C, Nunes JRC, et al. Choline transport links macrophage phospholipid metabolism and inflammation. J Biol Chem 2018;293:11600-11611.

23. Masselli G, Monti R, Guida M, Gualdi G. Giant schmorl's node may cause high uptake and mimic a bone metastasis on ${ }^{18} \mathrm{~F}$-Choline positron emission tomography/computed tomography. World J Nucl Med 2015;14:140-141.

24. Gu CN, Hunt CH, Lehman VT, Johnson GB, Diehn FE, Schwartz KM, et al. Benign fibrous dysplasia on $\left[{ }^{11} \mathrm{C}\right]$ choline PET: a potential mimicker of disease in patients with biochemical recurrence of prostate cancer. Ann Nucl Med 2012;26:599-602.

25. Wondergem M, van der Zant FM, van der Ploeg T, Knol RJ. A literature review of ${ }^{18} \mathrm{~F}$-fluoride PET/CT and ${ }^{18} \mathrm{~F}$-choline or ${ }^{11} \mathrm{C}$-choline PET/CT for detection of bone metastases in patients with prostate cancer. Nucl Med Commun 2013;34:935-945.

26. Florimonte L, Orunesu E, Castellani M, Longari V, Cortelezzi A. ${ }^{18} \mathrm{~F}$-Choline PET/CT-positive lytic bone lesions in prostate cancer and accidental myeloma detection. Clin Nucl Med 2016;41:394-396.

27. Sheldon JA, Yap KK, Taubman KL, Schlicht SM. Prevalence of non ${ }^{18} \mathrm{~F}$-fluorodeoxyglucose-avid incidental findings of clinical significance on whole body positron emission tomography/computed tomography: a review of 500 consecutive cases. J Med Imaging Radiat Oncol 2018;62:194-202.

28. Guo HH, Moradi F, lagaru A. Clinical significance of extraskeletal computed tomography findings on ${ }^{18} \mathrm{~F}-\mathrm{NaF}$ PET/CT performed for osseous metastatic disease evaluation. Nucl Med Commun 2016;37:975982.

29. Kan $\mathrm{H}$, van der Zant FM, Wondergem M, Knol RJJ. Incidental extra-cardiac findings on ${ }^{13} \mathrm{~N}$-ammonia myocardial perfusion PET/CT. J Nucl Cardiol 2017;24:1860-1868.

30. MacMahon H, Naidich DP, Goo JM, Lee KS, Leung ANC, Mayo JR, et al. Guidelines for management of incidental pulmonary nodules detected on CT images: from the Fleischner Society 2017. Radiology 2017;284:228-243.

31. Berland LL, Silverman SG, Gore RM, Mayo-Smith WW, Megibow AJ, Yee J, et al. Managing incidental findings on abdominal CT: white paper of the ACR incidental findings committee. J Am Coll Radiol 2010;7:754-773. 


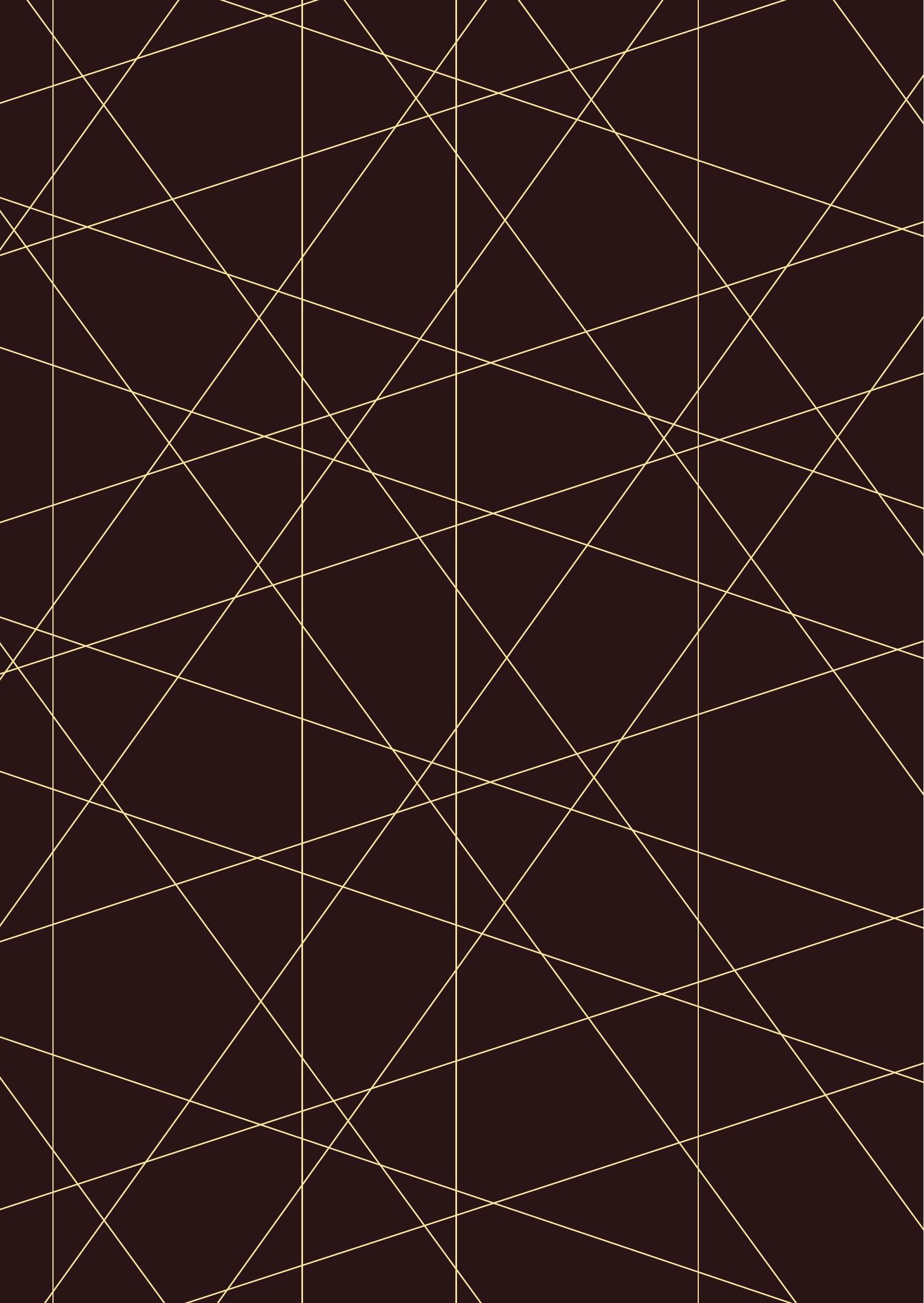




\section{PART 4}

Summary, discussion and future perspectives 


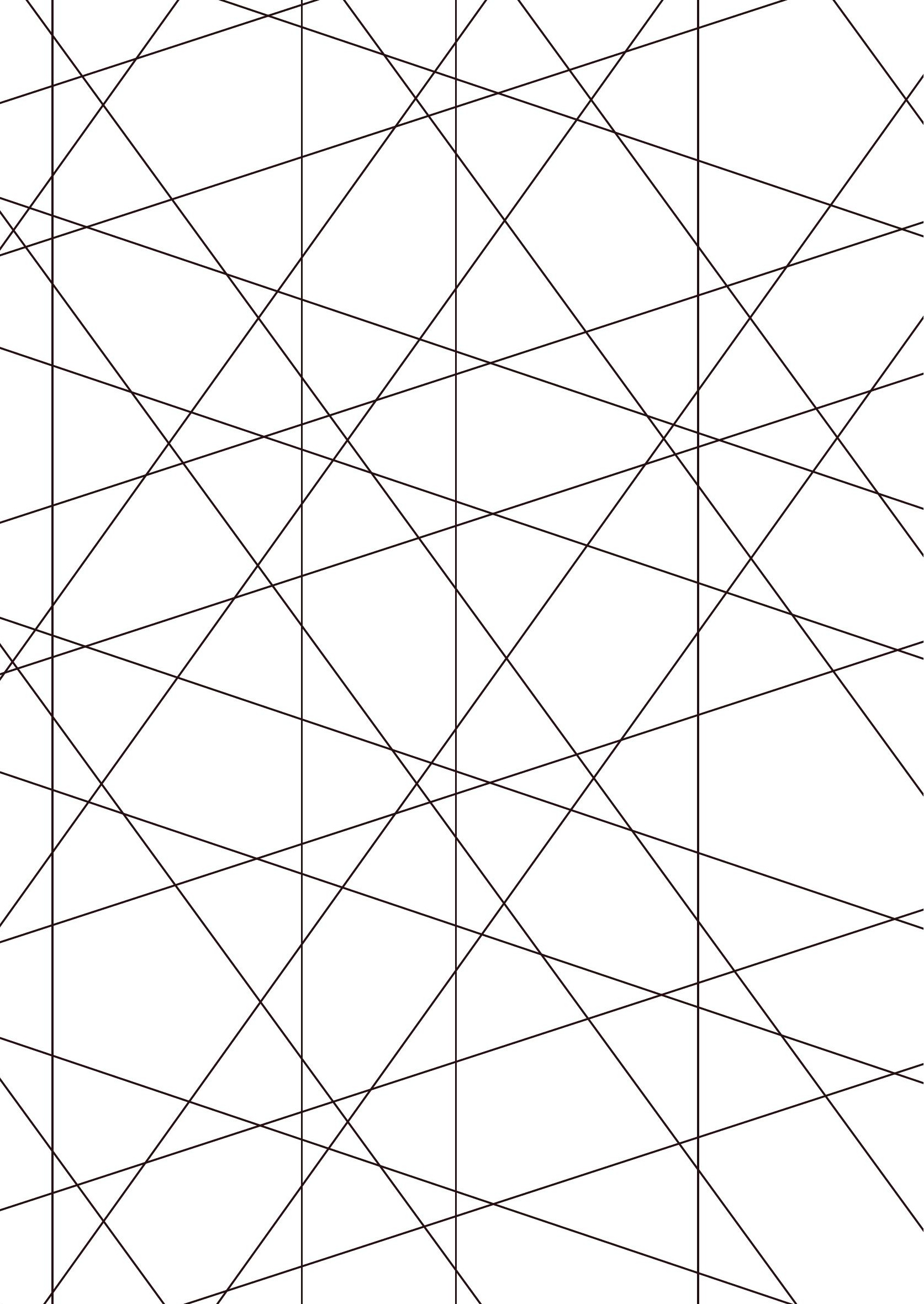




\section{CHAPTER 7}

Thesis summary 
Accurate preoperative localization of hyperfunctioning parathyroid glands is important in patients with primary hyperparathyroidism who are planned for parathyroidectomy, especially since minimally invasive surgical procedures are regularly used nowadays. An upcoming and promising imaging technique to localize hyperfunctioning parathyroid glands is PET/CT with ${ }^{18} \mathrm{~F}$-fluorocholine $\left({ }^{18} \mathrm{~F}-\mathrm{FCH}\right)$; however, the scientific evidence is still relatively sparse. The objectives of this thesis were to critically review the current scientific literature related to ${ }^{18} \mathrm{~F}-\mathrm{FCH}$ PET/CT, to further specify scan acquisition protocols and to explore the clinical implications of parathyroid localization using ${ }^{18} \mathrm{~F}-\mathrm{FCH}$ PET/CT.

In chapter 2, a comprehensive literature review was performed in which 11 studies on ${ }^{18} \mathrm{~F}-\mathrm{FCH}$ PET/CT parathyroid localization were included. Although the pooled performance data demonstrated high parathyroid lesion detection rates (94-97\%), there was a considerable heterogeneity among the published studies, the level of evidence was moderate and we concluded that further research was needed.

Since different scan acquisition protocols have been used throughout literature without consensus about the optimal protocol, several aspects involving scan acquisition parameters were studied in the following two chapters. Because some hyperfunctioning parathyroid glands are known to show fast wash-out of the tracer, they can be missed on regular imaging at 60 min post-injection (p.i.) of ${ }^{18} \mathrm{~F}-\mathrm{FCH}$. In chapter 3, we studied the value of an additional early scan at 5 min p.i. which was compared with standard late images (60 min p.i.). In a cohort of 64 patients with histopathologically proven parathyroid adenomas or hyperplasia, $20 \%$ was better visualized at the early time point, in $6 \%$ visualization was best at 60 min p.i. and in $2 \%$ the gland was exclusively visualized on late images. With demonstration of these different wash-out patterns, we concluded that dual-time-point images can be of added value in localizing parathyroid glands.

Moreover, parathyroid detection can be complicated by ${ }^{18} \mathrm{~F}-\mathrm{FCH}$ activity in surrounding structures such as the thyroid gland, obscuring parathyroid glands, or uptake in lymph nodes, a possible cause for false positive results. In chapter $\mathbf{4}$, the value of dynamic imaging to distinguish parathyroid glands from other structures with ${ }^{18} \mathrm{~F}-\mathrm{FCH}$ uptake was studied and, additionally, these data were analyzed to determine the optimal scan acquisition time points. A total of 101 dynamic scans in patients with histopathologically proven parathyroid adenomas were analyzed. Differentiation of a parathyroid adenoma from active lymph nodes was best in the first 5 min p.i., but could not definitively discriminate between the two structures. The optimal time point to distinguish a parathyroid adenoma from thyroid activity was after $10 \mathrm{~min}$. All in all, dynamic scanning contributed to characterization of hyperfunctioning parathyroid glands and determining optimal scan acquisition time points. 
To study ${ }^{18} \mathrm{~F}$-FCH PET/CT as a first-line imaging modality in our own clinical practice, scan performance was evaluated in a large patient cohort consisting of 271 patients with primary hyperparathyroidism, presented in chapter 5. In this cohort 139 patients underwent parathyroidectomy and the calculated detection rate of ${ }^{18} \mathrm{~F}-\mathrm{FCH}$ PET/CT was $90 \%$ and $96 \%$, in a per lesion-based and a per patient-based analysis, respectively. We concluded that the use of ${ }^{18} \mathrm{~F}-\mathrm{FCH}$ PET/CT as a first-line imaging modality is suitable in preoperative planning of parathyroid surgery.

Incidental findings are regularly detected on ${ }^{18} \mathrm{~F}-\mathrm{FCH}$ PET/CT for parathyroid imaging, but frequency and relevance are undetermined. Therefore, in the study presented in chapter 6, $388{ }^{18} \mathrm{~F}-\mathrm{FCH}$ PET/CT scans were reviewed for abnormalities unrelated to the parathyroid glands and were correlated with follow-up data. Incidental findings were detected on $58 \%$ of the scans, abnormally increased ${ }^{18} \mathrm{~F}-\mathrm{FCH}$ uptake was detected in $22 \%$ of the patients and malignant lesions were detected in 10 patients (2.6\%). Especially breast cancer was a frequently detected incidentaloma in this particular patient population in which women are typically over-represented.

In conclusion, the results of the studies presented in this thesis are in line with the available scientific literature on ${ }^{18} \mathrm{~F}-\mathrm{FCH}$ PET/CT parathyroid imaging and demonstrate superior performance of this imaging technique to localize hyperfunctioning parathyroid glands. Moreover, the findings in this thesis contribute to optimization of scan acquisition protocols and give a better insight into the frequency and relevance of incidental findings on ${ }^{18} \mathrm{~F}-\mathrm{FCH}$ PET/CT scans. Finally, although some aspects of this novel imaging technique could be further clarified, the expectation is that in the future ${ }^{18} \mathrm{~F}-\mathrm{FCH}$ PET/CT will have a prominent role in parathyroid imaging. 


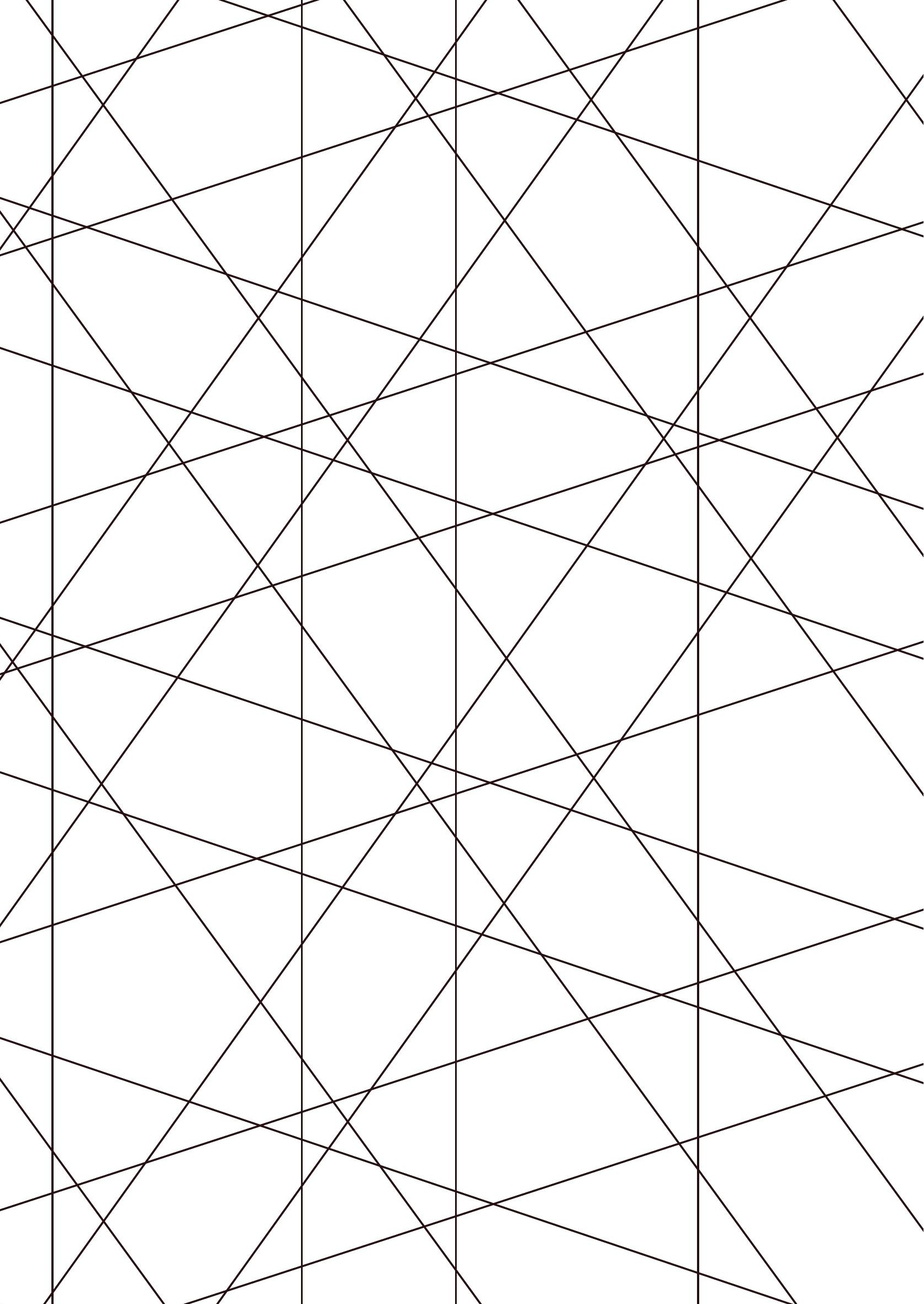




\section{CHAPTER 8}

General discussion 


\section{Uptake mechanism of ${ }^{18} \mathrm{~F}$-fluorocholine}

The exact underlying mechanisms for increased choline uptake in hyperfunctioning parathyroid glands have not yet been fully elucidated. Choline is an essential precursor for the biosynthesis of phospholipids, such as phosphatidylcholine, which are important components of cell membranes. In fact, cells cannot proliferate in the absence of choline.[1] Choline metabolism has been extensively studied in cancer. The majority of cancer cells demonstrate abnormal choline metabolism resulting in increased levels of intracellular choline. These cells also show increased amounts of choline products such as phosphorylcholine, which is formed by phosphorylation of choline by the enzyme choline kinase, which is the first step in the synthesis of phosphatidylcholine.[2] Identified molecular causes for increased choline concentration in cancer cells include increased choline kinase activity, increased choline kinase expression, higher rate of choline transport and increased activity of several other enzymes such as phosphatidylcholinespecific phospholipase.[2]

The known up-regulation of choline uptake and phosphorylation in malignancies stimulated the development of choline analogues labeled with positron emitting isotopes for cancer imaging with PET in the late 1990's. ${ }^{11} \mathrm{C}$-labeled choline $\left({ }^{11} \mathrm{C}-\mathrm{CH}\right)$ was initially developed and has been successfully used for cancer imaging, especially in prostate cancer.[3] Subsequently, ${ }^{18} \mathrm{~F}$-labeled tracers became of interest because of the favorable physical properties of ${ }^{18} \mathrm{~F}$ including its longer half-life (110 min versus 20 min for ${ }^{11} \mathrm{C}$ ) making it more suitable for transportation and its shorter positron range ( $2.4 \mathrm{~mm}$ versus $4.2 \mathrm{~mm}$ for ${ }^{11} \mathrm{C}$ ) which leads to higher spatial resolution of PET images.[4] Additionally, ${ }^{18} \mathrm{~F}$-labeled tracers show less tissue clearance compared to ${ }^{11} \mathrm{C}-\mathrm{CH}$ due to specific metabolic trapping of ${ }^{18} \mathrm{~F}$-labeled choline through phosphorylation and less oxidation. [5] Moreover, it has been demonstrated that the ${ }^{18} \mathrm{~F}$-tracer is not only phosphorylated but is actually incorporated into the cellular membrane as phosphatidylcholine.[6] Different ${ }^{18} \mathrm{~F}$-labeled analogues were developed, but the analogue ${ }^{18} \mathrm{~F}$-fluoro(methyl)choline $\left({ }^{18} \mathrm{~F}\right.$ $\mathrm{FCH}$ ), which was used in the studies presented in this thesis, demonstrated the best properties, such as better acceptance by choline kinase and higher uptake in prostate cancer cells in vitro.[7] Subsequent clinical studies also suggested superiority of ${ }^{18} \mathrm{~F}$ $\mathrm{FCH}$ over other choline analogues. However, in vivo studies directly comparing these radiopharmaceuticals are not available.[8]

Increased accumulation of ${ }^{18} \mathrm{~F}-\mathrm{FCH}$ in hyperfunctioning parathyroid glands was incidentally detected on ${ }^{18} \mathrm{~F}-\mathrm{FCH}$ PET/CT for prostate cancer detection.[9] Uptake mechanisms for this phenomenon are probably similar to those in cancer. For 
example, increased choline kinase activity has also been demonstrated in other benign tumors such as adenomas of the colon.[10] Higher amounts of choline metabolites in hyperfunctioning parathyroid cells, which have been found with nuclear magnetic resonance spectroscopy studies, also support this theory.[11] Moreover, a significant correlation between ${ }^{18} \mathrm{~F}-\mathrm{FCH}$ uptake and cell proliferation markers such as $\mathrm{Ki}-67$ expression in parathyroid cells has been demonstrated.[12]

\section{Update and discussion of the scientific literature on ${ }^{18} \mathrm{~F}$-fluorocholine PET/CT for parathyroid localization}

Since it was recognized that PET with radiolabeled choline can visualize hyperfunctioning parathyroid glands, the diagnostic value of this imaging method has been extensively studied. The systematic review presented in chapter $\mathbf{2}$ of this thesis was conducted with a search update until March 2018 and comprised eleven independent studies. Nine of those studies were eligible for extraction of performance data, which resulted in a pooled detection rate of $97 \%$ on a per patient-based analysis and $94 \%$ on a per lesion-based analysis. The detection rate was defined as the number of true-positives divided by (true-positives + false-negatives). Throughout the literature on ${ }^{18} \mathrm{~F}-\mathrm{FCH}$ PET/CT detection rate is used interchangeably with sensitivity and is calculated the same way. However, we used detection rate because the real number of false-negatives is often unknown, which impairs calculation of the true sensitivity. Several additional cohort studies have been published since our systematic review. An updated overview of all cohort studies on ${ }^{18} \mathrm{~F}$-FCH PET/CT for parathyroid localization is presented in Table 1. In general, the results of more recent studies are in line with the studies included in the systematic review. One of these more recent studies is our own cohort study presented in chapter 5 of this thesis, which comprises one of the largest cohorts presently available. This study showed detection rates of $96 \%$ on a per patient-based analysis and $90 \%$ on a per lesionbased analysis for ${ }^{18} \mathrm{~F}-\mathrm{FCH}$ PET/CT as a first-line imaging modality. Two other systematic reviews were published around the same period as our review, both including a metaanalysis.[13,14] Later on, in 2019, a systematic review was published by Boccalatte et al.[15]; however, they reported no pooled results. Most recently, a systematic review by Evangelista et al.[18] comprising 18 individual studies up to December 2019 was published. All these reviews are generally in line with our own review showing pooled detection rates of $\geq 90 \%$. An overview of all published systematic reviews with reported results on ${ }^{18} \mathrm{~F}-\mathrm{FCH}$ PET/CT parathyroid localization is presented in Table 2. 


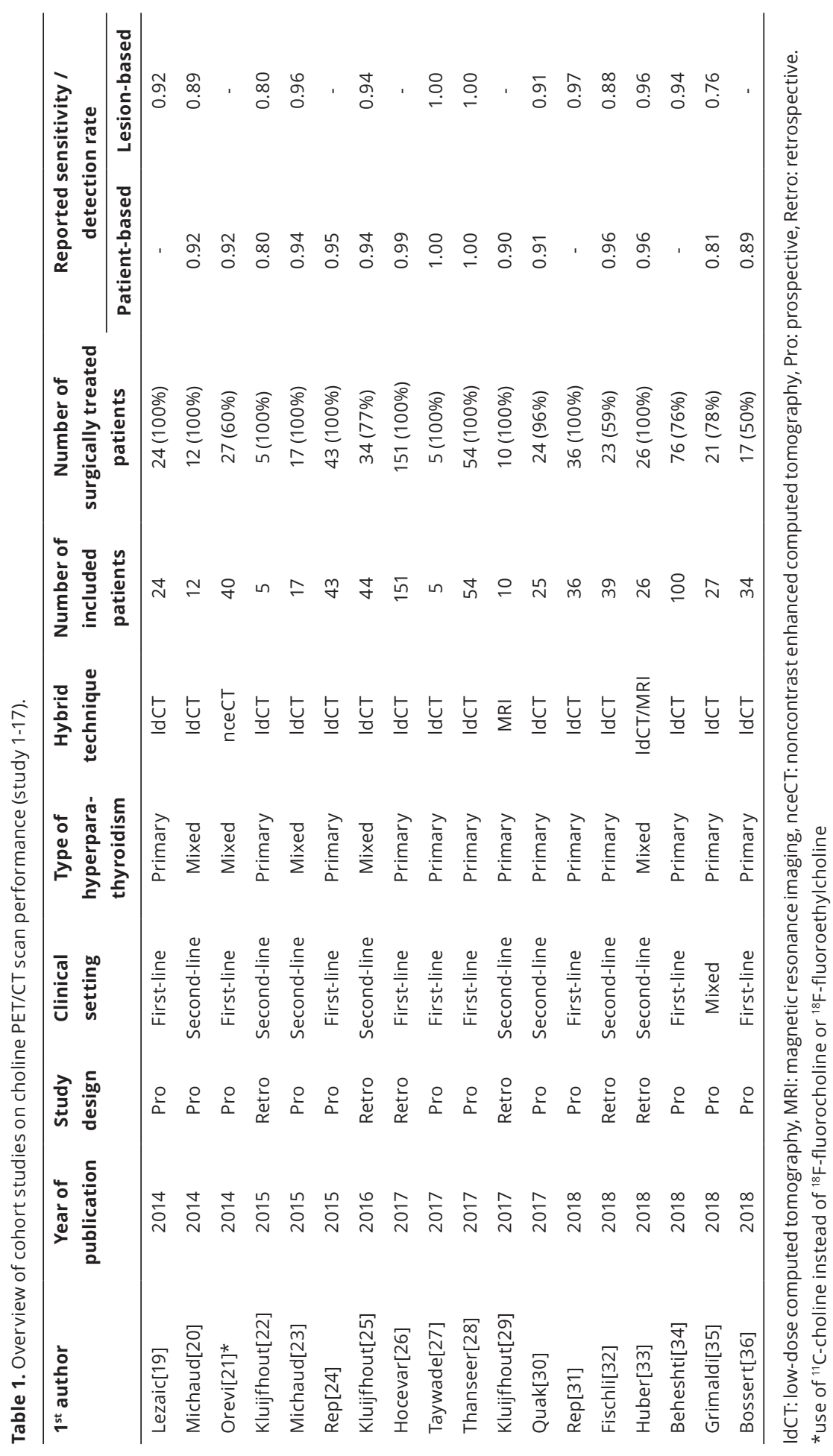




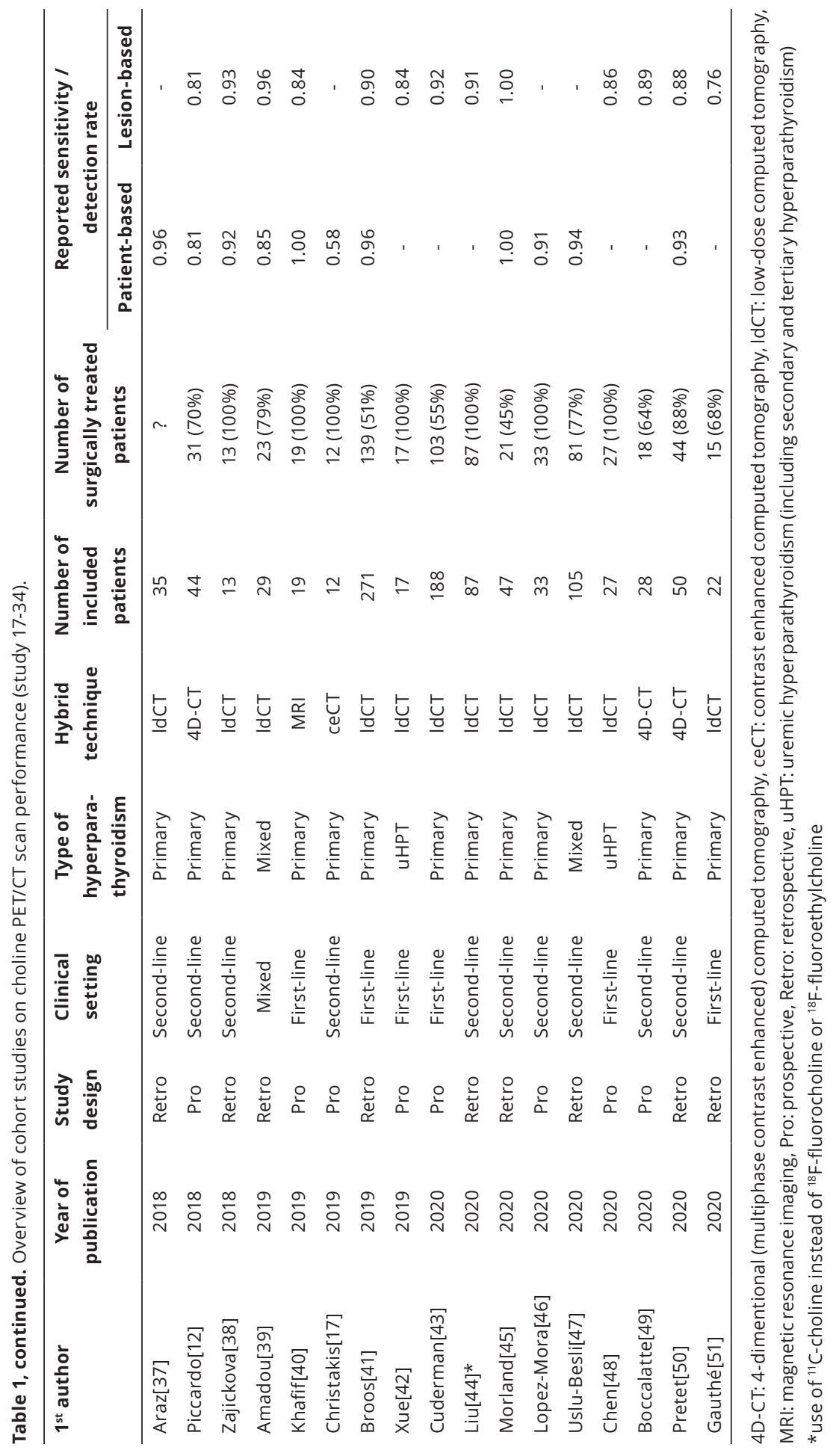


Table 2. Systematic reviews.

\begin{tabular}{|c|c|c|c|c|c|c|}
\hline \multirow[b]{2}{*}{ Author } & \multirow[b]{2}{*}{$\begin{array}{c}\text { Year of } \\
\text { publication }\end{array}$} & \multirow[b]{2}{*}{$\begin{array}{l}\text { Inclusion } \\
\text { until }\end{array}$} & \multirow{2}{*}{$\begin{array}{l}\text { Number } \\
\text { of studies } \\
\text { included }\end{array}$} & \multirow[b]{2}{*}{$\begin{array}{l}\text { Meta- } \\
\text { analysis }\end{array}$} & \multicolumn{2}{|c|}{$\begin{array}{c}\text { Pooled sensitivity / } \\
\text { detection rate }\end{array}$} \\
\hline & & & & & $\begin{array}{l}\text { Patient } \\
\text { based }\end{array}$ & $\begin{array}{l}\text { Lesion } \\
\text { based }\end{array}$ \\
\hline Kim et al.[14] & 2018 & Apr'18 & 8 & yes & - & 0.90 \\
\hline Broos et al.[52] & 2019 & Mar'18 & 9 & no & 0.97 & 0.94 \\
\hline Treglia et al.[13] & 2019 & May'18 & 14 & yes & 0.95 & 0.92 \\
\hline Boccalatte et al.[15] & 2019 & Nov'18 & 16 & no & - & - \\
\hline Evangelista et al.[18] & 2020 & Dec'19 & 18 & no & 0.94 & 0.91 \\
\hline
\end{tabular}

As shown by several systematic reviews and meta-analyses; the main limitation of the presently available literature is the heterogeneity between the published studies. This is caused by several factors, including: the clinical setting in which ${ }^{18} \mathrm{~F}-\mathrm{FCH}$ PET/CT is used (as a first-line or second-line imaging modality), patient population (only patients with primary hyperparathyroidism or also patients with other parathyroid disorders), study design (prospective or retrospective), used tracer $\left({ }^{18} \mathrm{~F}-\mathrm{FCH}\right.$ or $\left.{ }^{11} \mathrm{C}-\mathrm{CH}\right)$ and type of hybrid imaging technique (PET with low-dose CT, contrast enhanced CT or MRI). Each of these factors will be briefly discussed here.

Clinical setting: In clinical practice, ${ }^{18} \mathrm{~F}-\mathrm{FCH}$ PET/CT for parathyroid localization can be used in various ways. The scan can be used as a second-line imaging modality when conventional imaging (usually ${ }^{99 m}$ Tc-sestamibi scintigraphy and/or ultrasonography) is negative or inconclusive, or it can be used as a first-line imaging modality without prior imaging. The use of ${ }^{18} \mathrm{~F}-\mathrm{FCH}$ PET/CT has been studied in both settings. If used as a second-line imaging method, the detection rate can be expected to be lower due to preselection of patients with parathyroid glands which are harder to visualize, such as small adenomas. In the recent meta-analysis by Treglia et al.[13], a subgroup analysis was presented by patient type, which showed lower diagnostic performance in the patient group with inconclusive conventional imaging ( $85 \%$ positive scans) compared to patients without prior imaging ( $94 \%$ positive scans). In our own cohort study, we exclusively studied the technique as a first-line imaging modality.

Patient population: Most studies only included patients with primary hyperparathyroidism, while several others also studied patients with secondary and tertiary hyperparathyroidism. For cohorts including patients with secondary and tertiary hyperparathyroidism, a lower detection rate is expected because it often concerns multiglandular disease / hyperplasia with smaller parathyroid glands. These are conditions for which conventional imaging such as ${ }^{99 \mathrm{~m} T c-s e s t a m i b i}$ scintigraphy is 
known to have a lower sensitivity.[16] Regarding ${ }^{18} \mathrm{~F}-\mathrm{FCH}$ PET/CT, Treglia et al.[13] reported a similar diagnostic performance for studies focusing only on primary hyperparathyroidism compared with mixed cohorts; however, the number of nonprimary hyperparathyroidism patients in those studies was relatively small, possibly obscuring effects on detection rate. Our own cohort study only comprised patients with primary hyperparathyroidism. The use of ${ }^{18} \mathrm{~F}-\mathrm{FCH}$ PET/CT in other types of hyperparathyroidism will be further discussed later in this chapter.

Study design: The majority of the published studies on ${ }^{18} \mathrm{~F}-\mathrm{FCH}$ PET/CT parathyroid localization have a prospective study design with the remaining of the studies, including our own cohort study, a retrospective study design. Both study designs have their pros and cons and will be discussed in more detail later. Nevertheless, the results of the available prospective studies are generally in line with the results of the retrospective studies, which is further expressed in the meta-analysis by Treglia et al.[13] which showed no difference in the diagnostic performance between the two study designs.

Tracer: The vast majority of available studies were performed using ${ }^{18} \mathrm{~F}-\mathrm{FCH}$, the tracer that was also used in the studies presented in this thesis; two studies used ${ }^{11} \mathrm{C}-\mathrm{CH}$; and only one study explicitly mentioned the use of a ${ }^{18} \mathrm{~F}$-labeled choline tracer other than ${ }^{18} \mathrm{~F}-\mathrm{FCH}$ (Christakis et al.[17] reported the use of ${ }^{18} \mathrm{~F}$-fluoroethylcholine). In our own systematic review, studies with all available choline tracers were included, although some systematic reviews excluded studies that used ${ }^{11} \mathrm{C}-\mathrm{CH}$ instead of ${ }^{18} \mathrm{~F}-\mathrm{FCH} .[14,15,18]$ Theoretically, ${ }^{18} \mathrm{~F}-\mathrm{FCH}$ has superior properties over other choline tracers, as was described in the former paragraph. However, no studies directly comparing different choline tracers are available concerning parathyroid imaging and it is likely that choline tracers other than ${ }^{18} \mathrm{~F}-\mathrm{FCH}$ can be effectively used if ${ }^{18} \mathrm{~F}-\mathrm{FCH}$ is not available.

Hybrid imaging technique: ${ }^{18} \mathrm{~F}-\mathrm{FCH}$ PET is usually combined with low-dose CT for attenuation correction and anatomical localization but can also be combined with more sophisticated techniques such as higher-dose contrast enhanced CT or MRI. These techniques yield additional anatomic information which could lead to higher sensitivity and specificity. No subgroup analyses that differentiated between these different approaches were found in the present literature. In the studies presented in this thesis ${ }^{18} \mathrm{~F}-\mathrm{FCH}$ PET was combined with low-dose CT. In the following paragraphs, the other hybrid techniques are further discussed. 
Although present data is heterogeneous, the results of ${ }^{18} \mathrm{~F}-\mathrm{FCH}$ PET/CT imaging for parathyroid localization are consistently favorable throughout the literature, as compared to conventional imaging. This applies for both second-line imaging, when conventional imaging is inconclusive, as well as for first-line imaging with ${ }^{18} \mathrm{~F}-\mathrm{FCH}$ PET/CT replacing conventional methods, as has been demonstrated in chapter $\mathbf{5}$ of this thesis.

\section{Other imaging modalities for parathyroid localization}

Besides ${ }^{18} \mathrm{~F}-\mathrm{FCH} \mathrm{PET} / \mathrm{CT}$, several other imaging modalities can be used to localize enlarged or hyperfunctioning parathyroid glands. One of the most frequently used imaging modalities is parathyroid scintigraphy. In current clinical practice different combinations of techniques are applied for parathyroid scintigraphy. Most use ${ }^{99 m T C}$ sestamibi, in which the gamma ray emitting radioisotope ${ }^{99 \mathrm{~m}} \mathrm{Tc}$ is bound to six (sesta) methoxyisobutylisonitrile (mibi) ligands. This complex is lipophilic and cationic and especially accumulates in cells with high numbers of negatively charged mitochondria, such as parathyroid cells. The tracer also accumulates in the thyroid gland, but with lower uptake per gram of tissue and with faster wash-out. Therefore, sequential images can visualize hyperfunctioning parathyroid glands (wash-out or dual-phase protocol). Another method is a subtraction technique, using a tracer that only visualizes the thyroid gland (such as ${ }^{99 \mathrm{~m} T c-p e r t e c h n e t a t e ~ o r}{ }^{123}$ /-iodine) and subsequently subtract these images from the ${ }^{99 m}$ Tc-sestamibi images (subtraction or dual-tracer protocol). The wash-out and subtraction protocols can also be combined to improve the sensitivity and both can be performed planar as well as tomographic with singe-photon emission computed tomography (SPECT) to acquire 3-dimensional images, also increasing sensitivity. Adding CT to SPECT (SPECT/CT) provides additional anatomical detail and further increases accuracy. Variability of used scanning protocols makes comparison of published studies on parathyroid scintigraphy difficult. In a meta-analysis by Wong et al.[53], a sensitivity of $70 \%$ was calculated for planar scintigraphy, $74 \%$ for SPECT and $86 \%$ for SPECT/CT. Several studies directly compared ${ }^{99 m} \mathrm{Tc}$-sestamibi scintigraphy with ${ }^{18} \mathrm{~F}-\mathrm{FCH}$ PET/CT in which ${ }^{18} \mathrm{~F}-\mathrm{FCH}$ PET/CT consistently yielded higher accuracy (Table 3).

${ }^{99 m T c-s e s t a m i b i ~ s c i n t i g r a p h y ~ i s ~ o f t e n ~ c o m b i n e d ~ w i t h ~ u l t r a s o n o g r a p h y ~(U S) . ~ U S ~ i t s e l f ~}$ has a lower sensitivity but has low costs, is non-invasive, has no radiation and can be performed by the surgeon preoperatively. Furthermore, it is complementary to ${ }^{99 m T c-}$ sestamibi scintigraphy and accuracies of $>90 \%$ have been described when the two modalities are combined.[54,55] However, accuracy of the technique is highly operatordependent and diagnostic performance is reduced for small adenomas, multiglandular disease and in the presence of goiter. In one study sensitivity of 94-100\% in patients with 
normal thyroid dropped to 54-84\% in the presence of thyroid nodules.[56] Moreover, US has very limited use for detection of ectopic parathyroid glands. Accuracy could be increased with the use of new US techniques such as elastography and contrast enhanced US, as was demonstrated in proof-of-concept studies.[57,58]

Table 3. Studies directly comparing ${ }^{18} \mathrm{~F}-\mathrm{FCH}$ PET/CT with ${ }^{99 \mathrm{mTC}} \mathrm{T}-\mathrm{sestamibi}$ scintigraphy.

\begin{tabular}{|c|c|c|c|c|}
\hline \multirow[b]{2}{*}{$1^{\text {st }}$ author } & \multirow[b]{2}{*}{ Year } & \multirow{2}{*}{$\begin{array}{l}{ }^{99 m} \text { Tc-sestamibi } \\
\text { scintigraphy } \\
\text { acquisition protocol }\end{array}$} & \multicolumn{2}{|c|}{$\begin{array}{c}\text { Reported sensitivity / } \\
\text { detection rate* }\end{array}$} \\
\hline & & & $\begin{array}{l}\text { 99mTc-sestamibi } \\
\text { scintigraphy }\end{array}$ & ${ }^{18} \mathrm{~F}-\mathrm{FCH} \mathrm{PET} / \mathrm{CT}$ \\
\hline \multirow[t]{3}{*}{ Lezaic[19] } & 2014 & Subtraction & 0.64 & 0.92 \\
\hline & & Wash-out & & \\
\hline & & SPECT/CT & & \\
\hline \multirow[t]{3}{*}{ Orevi[21] } & 2014 & Subtraction & 0.88 & 0.92 \\
\hline & & Wash-out & & \\
\hline & & SPECT/CT & & \\
\hline \multirow[t]{2}{*}{ Thanseer[28] } & 2017 & Wash-out & 0.81 & 1.00 \\
\hline & & SPECT/CT & & \\
\hline \multirow[t]{2}{*}{ Beheshti[34] } & 2018 & Wash-out & 0.61 & 0.94 \\
\hline & & SPECT/CT & & \\
\hline \multirow[t]{3}{*}{ Grimaldi[35] } & 2018 & Subtraction & $0.15-0.41$ & 0.76 \\
\hline & & Wash-out & & \\
\hline & & SPECT/CT & & \\
\hline \multirow[t]{3}{*}{ Bossert[36] } & 2018 & Subtraction & 0.17 & 0.89 \\
\hline & & Wash-out & & \\
\hline & & SPECT/CT & & \\
\hline \multirow[t]{2}{*}{ Araz[37] } & 2018 & Wash-out & 0.78 & 0.96 \\
\hline & & SPECT/CT & & \\
\hline \multirow[t]{3}{*}{ Amadou[39] } & 2019 & Subtraction & 0.50 & 0.96 \\
\hline & & Wash-out & & \\
\hline & & SPECT/CT & & \\
\hline Khafif[40] & 2019 & Not reported & 0.74 & 0.84 \\
\hline \multirow[t]{2}{*}{ Xue[42] } & 2019 & Wash-out & 0.63 & 0.84 \\
\hline & & SPECT/CT & & \\
\hline \multirow[t]{3}{*}{ Cuderman[43] } & 2020 & Subtraction & 0.65 & 0.92 \\
\hline & & Wash-out & & \\
\hline & & SPECT/CT & & \\
\hline \multirow[t]{2}{*}{ Chen[48] } & 2020 & Wash-out & 0.55 & 0.86 \\
\hline & & SPECT/CT & & \\
\hline \multirow[t]{3}{*}{ Gauthé[51] } & 2020 & Subtraction & 0.61 & 0.76 \\
\hline & & Wash-out & & \\
\hline & & SPECT/CT & & \\
\hline
\end{tabular}

*lesion-based, if not available patient-based 
Contrast enhanced CT may also be used to visualize hyperfunctioning parathyroid glands and has advantages such as precise anatomical localization, the ability to detect ectopic glands and its wide availability. 4D-CT is a promising scanning technique, where 4D implies the addition of the $4^{\text {th }}$ dimension "time", which is achieved by combination of different contrast phases, usually a pre-contrast scan, an arterial phase scan (25-30 s after contrast administration), a venous phase scan (approximately $30 \mathrm{~s}$ later) and sometimes a delayed phase scan (another $30 \mathrm{~s}$ later). Detection of parathyroid lesions are based on changes in enhancement over time: parathyroid adenomas typically have low attenuation on noncontrast images, peak enhancement during the arterial phase and wash-out in subsequent phases. In a systematic review by Kluijfhout et al.[59], the addition of contrast phases increased sensitivity from $68 \%$ to $71 \%, 76 \%$ and $80 \%$ for one, two or three additional phases, respectively. A main disadvantage of 4D-CT is the additional radiation burden to the patient with each extra phase, although this may be reduced with modern dual-energy CT scanners that enable reconstruction of noncontrast images from contrast enhanced images. Several studies demonstrated superiority of 4D-CT over ${ }^{99 \mathrm{~m} T c-s e s t a m i b i}$ scintigraphy (sensitivities of 79-82\% for 4D-CT vs. $49-58 \%$ for scintigraphy). $[60,61]$ A limited number of studies also directly compared 4D-CT with ${ }^{18} \mathrm{~F}-\mathrm{FCH}$ PET/CT in the same patient group. In a small case-series by Taywade et al.[27] both 4D-CT and ${ }^{18} \mathrm{~F}-\mathrm{FCH}$ PET/CT had a $100 \%$ sensitivity as first-line imaging in five patients. Other available studies comparing 4D-CT and ${ }^{18} \mathrm{~F}-\mathrm{FCH}$ PET/CT used this for second-line imaging. A study by Amadou et al.[39] retrospectively compared ${ }^{18} \mathrm{~F}-\mathrm{FCH}$ PET/ CT and 4D-CT that were both performed in 20 patients. They demonstrated a lesionbased sensitivity of $96 \%$ for ${ }^{18} \mathrm{~F}-\mathrm{FCH}$ PET/CT versus $75 \%$ for 4D-CT. Two studies analyzed hybrid ${ }^{18} \mathrm{~F}-\mathrm{FCH}$ PET/4D-CT: Pretet et al.[50] observed a significantly better lesion-based $(n=54)$ sensitivity of $88 \%$ for ${ }^{18} \mathrm{~F}-\mathrm{FCH}$ PET vs. $66 \%$ for $4 \mathrm{D}-\mathrm{CT}(P=0.01)$. Integrated ${ }^{18} \mathrm{~F}-\mathrm{FCH}$ PET/4D-CT yielded a slightly higher sensitivity of $92 \%$, not significantly better than ${ }^{18} \mathrm{~F}$ FCH PET $(P=0.48)$. A prospective study by Piccardo et al.[12] demonstrated a significantly better performance of hybrid ${ }^{18} \mathrm{~F}-\mathrm{FCH}$ PET/4D-CT (sensitivity of $100 \%$ in 31 patients) compared with ${ }^{18} \mathrm{~F}$-FCH PET or 4D-CT separately $(P<0.05) .{ }^{18} \mathrm{~F}-\mathrm{FCH}$ PET demonstrated a higher sensitivity than $4 \mathrm{D}-\mathrm{CT}(80 \%$ vs. $74 \%)$ but no statistic comparison was reported. Boccalatte et al.[49] also studied ${ }^{18} \mathrm{~F}-\mathrm{FCH}$ PET/4D-CT and demonstrated a sensitivity of $89 \%$ but did not analyze ${ }^{18} \mathrm{~F}-\mathrm{FCH}$ PET and 4D-CT separately. Altogether, abovementioned data show generally better performance of ${ }^{18} \mathrm{~F}-\mathrm{FCH}$ PET/CT over 4D-CT in studies that directly compared the two modalities.

Magnetic resonance imaging (MRI) has also been studied for imaging of parathyroid glands and may be combined with PET in hybrid PET/MRI scanners. MRI has superior softtissue contrast as compared to $\mathrm{CT}$ and does not use ionizing radiation which is especially 
favorable in pregnant women or children. Conventional MRI (1.5T, without contrast enhancement) has a moderate sensitivity of approximately $80 \%$, but may be increased using stronger magnetic fields (3T) and contrast enhancement with gadolinium. A recent small cohort study demonstrated the promising ability of dynamic contrast-enhanced MRI (4D-MRI) with correct localization of a parathyroid adenoma in 34 out of 37 patients (92\%).[62] Additionally, 4D-MRI has been shown to accurately differentiate parathyroid glands from lymph nodes and adjacent thyroid gland.[63] Limitations are common MRI contraindications such as non-MRI compatible cardiac pacemakers and intracranial aneurysm clips, and the limited availability of 3T MRI scanners, specific coils and required software for dynamic image acquisition. All in all, parathyroid imaging using MRI seems promising but remains in a relatively early phase of development.

Several PET tracers other than ${ }^{18} \mathrm{~F}-\mathrm{FCH}$ have been studied for parathyroid imaging. ${ }^{11} \mathrm{C}$-methionine has been one of the most promising, with reported sensitivities ranging between $44 \%$ to $91 \%$ and a pooled estimate of 77\%.[64] PET/CT with ${ }^{18} \mathrm{~F}$-fluordeoxyglucose $\left({ }^{18} \mathrm{~F}-\mathrm{FDG}\right)$ has shown variable results: although Neumann et al.[65] published several articles with reported sensitivities up to $94 \%$, other studies showed poor sensitivity of 0 to 27\%.[66-68] Pilot studies with other PET tracers yielded no useful results; no significant uptake of ${ }^{18} \mathrm{~F}$-dihydroxyphenylalanine $\left({ }^{18} \mathrm{~F}\right.$-DOPA $)$ or ${ }^{18} \mathrm{~F}$-fluoroethyl-L-thyrosine $\left({ }^{18} \mathrm{~F}-\mathrm{FET}\right)$ was demonstrated in parathyroid adenomas.[69,70] Since the introduction of choline-based PET tracers to localize parathyroid glands, other PET tracers became of little relevance and interest in finding additional PET tracers for parathyroid detection declined.

Table 4. Comparison of meta-analyses on various imaging modalities used to localize parathyroid abnormalities.

\begin{tabular}{llccc}
\hline Imaging modality & \multicolumn{1}{c}{$\mathbf{1}^{\text {st author }}$} & $\begin{array}{c}\text { Year of } \\
\text { publication }\end{array}$ & $\begin{array}{c}\text { Number of } \\
\text { studies }\end{array}$ & $\begin{array}{c}\text { Pooled } \\
\text { sensitivity }\end{array}$ \\
\hline Choline PET/CT & Treglia[13] & 2019 & 14 & 0.95 \\
Sestamibi SPECT/CT & Wong[53] & 2015 & 24 & 0.86 \\
Methionine PET/CT & Kluijfhout[64] & 2016 & 14 & 0.77 \\
Ultrasonography & Cheung[71] & 2012 & 19 & 0.76 \\
CT (4D-CT) & Kluijfhout[59] & 2017 & $34(12)$ & $0.73(0.80)$ \\
MRI & & \multicolumn{3}{c}{ No meta-analysis available } \\
\hline
\end{tabular}

An overview of the diagnostic performance of the abovementioned imaging modalities is listed in Table 4. For each modality, pooled sensitivity was extracted from the metaanalysis with the highest number of included studies. No meta-analyses on MRI or PET tracers other than ${ }^{18} \mathrm{~F}-\mathrm{FCH}$ and ${ }^{11} \mathrm{C}$-methionine were available. According to this overview, PET/CT with choline-based tracers yield the highest sensitivity compared 
to other imaging modalities. Although studies on ${ }^{18} \mathrm{~F}-\mathrm{FCH}$ PET/CT parathyroid imaging are regularly subject to bias and heterogeneity as described in the former paragraph, studies with other imaging techniques have similar study designs and deal with the same limitations; therefore, it seems appropriate to compare those studies directly. One of the most promising modern imaging techniques next to ${ }^{18} \mathrm{~F}-\mathrm{FCH}$ PET/CT is 4D-CT. In the meta-analysis by Kluijfhout et al.[59] the reported sensitivity of 4D-CT is $80 \%$, lower than conventional scintigraphy with ${ }^{99 m}$ Tc-sestamibi (86\%).[53] Although, as mentioned before, several studies that compared these modalities directly, demonstrated superiority of 4D-CT over scintigraphy.[60,61] Nonetheless, in studies that directly compared 4D-CT with ${ }^{18} \mathrm{~F}-\mathrm{FCH} \mathrm{PET} / \mathrm{CT}$, the latter consistently performed better.[12,39,50]

\section{Practical benefits and drawbacks of ${ }^{18} \mathrm{~F}$-fluorocholine PET/CT}

Besides the superior detection rates described in the former paragraphs, ${ }^{18} \mathrm{~F}-\mathrm{FCH}$ PET/ CT for parathyroid localization has several other benefits over conventional scintigraphy, including shorter examination time, less patient preparation and less radiation exposure. However, cost-effectivity is unknown. Each of these issues will be discussed below.

Examination time: ${ }^{18} \mathrm{~F}-\mathrm{FCH}$ PET/CT has a shorter examination time as compared to parathyroid scintigraphy. Commonly, the patient is scanned at $60 \mathrm{~min}$ post ${ }^{18} \mathrm{~F}-\mathrm{FCH}$ p.i. with 2 bed positions of 2-10 min (depending on administered dose and sensitivity of the PET scanner). Additionally, some protocols require an early scan at approximately $5 \mathrm{~min}$ p.i.. A common scan protocol for ${ }^{99 m} \mathrm{Tc}$-sestamibi scintigraphy contains acquisition of static images at 10, 60 and 120 min p.i. (duration of 5 min per scan, wash-out protocol) and a SPECT/CT between $60-120$ p.i. (duration of $30 \mathrm{~min}$ ). $\mathrm{A}^{123}$ thyroid scintigraphy is acquired $24 \mathrm{~h}$ after oral administration of ${ }^{123}$ ( (subtraction protocol). The duration of an MRI can take up to 30 min, for US it is operator-dependent and CT takes the least time (several minutes and depending on the number of phases that are scanned).

Patient preparation: Prior to scanning with ${ }^{18} \mathrm{~F}-\mathrm{FCH} \mathrm{PET} / \mathrm{CT}$, there is no need to stop calcimimetic drugs or other medications with the exception of colchicine, of which it is advised to discontinue for $48 \mathrm{~h}$ because it may impair the normal metabolic choline pathway.[72] For ${ }^{99 m}$ Tc-sestamibi scintigraphy, need for discontinuation of possible interacting drugs is unclear; the American guidelines [73] do not advise to discontinue any medication but the European guidelines [74] advise to discontinue calcimimetics for at least 2 weeks before parathyroid imaging and active vitamin D therapy for at least 1 week. If subtraction scintigraphy with ${ }^{123}$ I is performed, also discontinuation of thyroid medication for 4-15 days and additionally absence of iodine saturation is recommended. 
Contrast media are needed for CT (iodine) and MRI (gadolinium). Therefore patients with allergies for these contrast media need to be prepared with cortisone and antihistamines, and patients with impaired renal function need to be prehydrated to prevent post contrast acute kidney injury. The tracers used in nuclear medicine techniques are of very low concentrations (nanomolar to picomolar concentration range); they normally do have no side-effects such as allergic reactions or acute kidney injury.

Radiation exposure: The radiation exposure of ${ }^{18} \mathrm{~F}-\mathrm{FCH}$ PET/CT is lower than ${ }^{99 \mathrm{mTC}-\mathrm{sestamibi}}$ scintigraphy, while CT induces the highest effective dose (Table 5). US and MRI give no radiation dose. Radiation exposure may be 3-4 times higher for 4D-CT compared with monophasic $\mathrm{CT}$, but can be reduced with new dual energy techniques, as mentioned before. Furthermore, although radiation exposure is relatively high for 4D-CT, the lifetime attributable risk of cancer from radiation exposure is considered negligible.[75]

Table 5. Radiation exposure

\begin{tabular}{lccc}
\hline Imaging modality & $\begin{array}{c}\text { Administered } \\
\text { activity (MBq) }\end{array}$ & $\begin{array}{c}\text { Effective dose conversion } \\
\text { factor (mSv/MBq) }\end{array}$ & $\begin{array}{c}\text { Effective dose } \\
\text { (mSv) }\end{array}$ \\
\hline${ }^{18}$ F-fluorocholine PET & 150 & $0.02^{(\mathrm{a})}$ & 3.0 \\
${ }^{11}$ C-choline PET & 370 & $0.0044^{(\mathrm{b})}$ & 1.6 \\
${ }^{11}$ C-methionine PET & 740 & $0.0084^{(\mathrm{c})}$ & 6.2 \\
${ }^{99 m T C-s e s t a m i b i ~ s c i n t i g r a p h y ~}$ & 700 & $0.009^{(\mathrm{d})}$ & 6.3 \\
${ }^{99 m T C-p e r t e c h n e t a t e ~ s c i n t i g r a p h y ~}$ & 100 & $0.013^{(\mathrm{d})}$ & 1.3 \\
${ }^{123}$ I scintigraphy & 18 & $0.22^{(\mathrm{d})}$ & 4.0 \\
Low-dose CT & - & - & $0.8^{(\mathrm{e})}$ \\
4D-CT & - & - & $10.4-28.7^{(\mathrm{f})}$ \\
MRI & - & - & 0 \\
Ultrasonography & - & - & 0 \\
\hline
\end{tabular}

Sources:

(a) A fourth addendum to ICRP Publication 53, 2013. (www.icrp.org)

(b) Tolvanen et al.[76]

(c) ICRP Publication 106 (addendum 3 to ICRP publication 53).[77]

(d) ICRP Publication 80 (addendum 2 to ICRP publication 53).[78]

(e) Rep et al.[31]

(f) Mahajan et al.[79] and Hoang et al.[75]

Cost and availability: Gamma cameras for scintigraphy are more commonly available than PET/CT scanners, although in the past decades PET/CT has become widely available in the Western world. For ${ }^{11} \mathrm{C}$-based tracers such as ${ }^{11} \mathrm{C}-\mathrm{CH}$, an on-site cyclotron is needed because of fast disintegration of the radio-isotope (half-life of $20 \mathrm{~min}$ ). ${ }^{18} \mathrm{~F}$-based tracers such as ${ }^{18} \mathrm{~F}-\mathrm{FCH}$, however, have a half-life of $110 \mathrm{~min}$ and are therefore more suitable for transportation. Currently, no cost-effectiveness analyses are available on ${ }^{18} \mathrm{~F}-\mathrm{FCH}$ PET/ 
CT for parathyroid imaging. As a result of differences in availability of PET/CT cameras, PET tracers and cyclotrons, costs vary greatly between countries, regions and even between hospitals in the same region. The costs for PET/CT are generally higher than for conventional scintigraphy or radiologic techniques; however, a number of aspects have led to a decrease in the costs of PET/CT scans. The use of modern PET cameras and more sophisticated reconstruction and postprocessing software has led to a decrease in the required doses of expensive PET tracers. Growing indications for PET tracers and further technical developments has made cyclotron facilities more cost-effective and there is an ongoing expansion of cyclotron facilities. Therefore, it is likely that the costs of PET tracers and hence PET/CT scans will decrease even more in the near future.

\section{Incidental findings on ${ }^{18} \mathrm{~F}$-fluorocholine PET/CT}

${ }^{18} \mathrm{~F}-\mathrm{FCH}$ PET/CT scans to localize hyperfunctioning parathyroid glands regularly demonstrate incidental findings due to nonspecific uptake of ${ }^{18} \mathrm{~F}-\mathrm{FCH}$. Uptake can be observed in other benign abnormalities including inflammatory lesions or in malignancies. In the study presented in chapter 6 of this thesis, incidental findings on $388{ }^{18} \mathrm{~F}-\mathrm{FCH}$ PET/CT scans for parathyroid localization performed in our center were analyzed. Abnormally increased ${ }^{18} \mathrm{~F}-\mathrm{FCH}$ uptake was detected in $22 \%$ of the patients. Malignancy was found in 10 patients (2.6\%) of which breast cancer was most common (7 patients). The high frequency of incidentally detected breast cancer on ${ }^{18} \mathrm{~F}-\mathrm{FCH}$ PET/CT was not demonstrated before, mainly because earlier studies on incidental findings with ${ }^{18} \mathrm{~F}-\mathrm{FCH}$ PET/CT concentrated on male cohorts with scans acquired for prostate cancer imaging. In those large prostate cancer cohorts, secondary malignancies were discovered on ${ }^{18} \mathrm{~F}-\mathrm{FCH}$ PET/CT in 1-1.5\% and on ${ }^{11} \mathrm{C}-\mathrm{CH}$ PET/CT in 2.6\%.[80-82] Interestingly, in a recent study on $2413{ }^{99 m}$ Tc-sestamibi SPECT/CT scans [83], 27\% of all patients demonstrated additional findings and 72 scans (3\%) revealed malignant or premalignant conditions, numbers that are roughly in line with the ${ }^{18} \mathrm{~F}-\mathrm{FCH}$ PET/CT cohort presented in this thesis. ${ }^{18} \mathrm{~F}-\mathrm{FCH}$ uptake in malignancies is explained by known upregulation of choline transport and choline kinase activity, as described in the first paragraph, and is probably also the reason for uptake in benign tumors such as adrenal adenomas and hyperplastic thyroid nodules that were found in our cohort. High ${ }^{18} \mathrm{~F}-\mathrm{FCH}$ uptake in inflammatory lesions, which was the most common cause for incidental ${ }^{18} \mathrm{~F}-\mathrm{FCH}$ uptake in our cohort, is probably related to a higher choline demand in macrophages.[84,85]

Although incidental findings are an inevitable effect of modern imaging technology, especially in techniques that image large parts of the body, our data show that these findings cannot easily be ignored. It is important to establish a solid explanation for 
unexpected uptake of the tracer, which may be found when ${ }^{18} \mathrm{~F}-\mathrm{FCH}$ uptake is correlated with findings on (low-dose) CT and clinical features. By using other hybrid techniques such as ${ }^{18} \mathrm{~F}-\mathrm{FCH}$ PET/4D-CT or PET/MRI anatomical correlation could be enhanced; however, the additional acquired data could also further increase the number of incidental findings. The results of our study can guide clinicians in follow-up strategies and help nuclear medicine physicians to interpret incidental findings.

\section{${ }^{18} \mathrm{~F}$-fluorocholine PET/CT acquisition protocol optimization}

Acquisition protocols that have been used for ${ }^{18} \mathrm{~F}-\mathrm{FCH}$ PET/CT vary considerably between published studies in the literature. Protocols usually include static image acquisition somewhere between 10-60 min after administration of the tracer. Additionally, several studies use an early acquisition and sometimes dynamic images are acquired. Rep et al.[24] reported on a triple-time-point protocol (5, 60 and 120 min p.i.) and concluded that the optimal scan time was at 60 min p.i.. At this time point, a higher accuracy was achieved compared to early images, whereas a similar accuracy was achieved compared to images acquired at 120 min p.i. (accuracy of $94.1 \%, 96.5 \%$ and $96.5 \%$ at 5, 60 and 120 min p.i., respectively). That research group also recognized incidental early wash-out of lesions and recommended additional early imaging if logistically possible.[24] Acquisition of early images has also been suggested by others since in some hyperfunctioning parathyroid glands early wash-out is observed.[86-88] Therefore, we evaluated a dual-time-point acquisition protocol with imaging at 5 and 60 min p.i., presented in chapter 3 of this thesis. Early imaging at 5 min p.i. yielded adequate visualization of hyperfunctioning parathyroid glands in most patients (89\%). However, in the other $11 \%$ of the cohort, the glands were best visualized at $60 \mathrm{~min} \mathrm{p.i.} \mathrm{due} \mathrm{to} \mathrm{tracer} \mathrm{wash-out} \mathrm{from}$ the thyroid gland at the late time point, which resulted better image contrast. On the other hand, we also observed cases with near complete tracer wash-out on late images (in $20 \%$ of the patients visualization of parathyroids was better on early images), showing that images acquired 5 and 60 min p.i. are complementary. The pathophysiological mechanism of fast wash-out from those glands is unclear but it could be related to high arterial perfusion (which is also regularly observed on contrast enhanced CT) with subsequently low retention i.e. phosphorylation of the tracer in the cells.

Tracer uptake over time can be studied with dynamic imaging, in which data is continuously acquired during a certain time frame and reconstructed to images at multiple time points. In the study presented in chapter $\mathbf{4}$ of this thesis, dynamic imaging was used to determine the optimal acquisition time point to differentiate a parathyroid adenoma from other ${ }^{18} \mathrm{~F}-\mathrm{FCH}$ accumulating structures. The first 2 min were unsuitable for 
diagnostic use due to blood pool activity. Best discrimination with active lymph nodes (a possible cause for false positive results) was before $5 \mathrm{~min}$ and best discrimination with the thyroid gland, whose activity can obscure nearby located parathyroids, was after 10 min. Therefore, a single optimal time-point that covered all the mentioned aspects could not be determined and we concluded that dynamic imaging could contribute in some cases. Another ability of dynamic imaging is the reconstruction of time-activity-curves. Certain characteristics of these time-activity curves could further help in differentiating parathyroid glands from lymph nodes, as was suggested by Prabhu et al.[87] who found a typical time-to-peak activity after 6 min for lymph nodes; however, this could not be reproduced in our own study. The main difference between the curves that was observed in our own study was the relatively stable activity over time in lymph nodes and rapidly decreasing activity in parathyroid glands.

Other aspects of PET/CT scanning protocols that can be altered are counting time per bed position, administered activity and scan range. The counting time per bed position (usually 2-10 min) and administered activity (usually between 100-300 MBq) are related to the sensitivity of the used PET/CT system and the quality of the scan that is aimed for. Throughout the literature, there seems to be a general consensus about the part of the body that should be imaged. Commonly, the scan ranges at least from the level of the skull base to the mediastinum to cover possible ectopic adenomas, which have been found anywhere along the carotid sheath to the pericardium.[89] Especially the inferior parathyroid glands, which during embryogenesis descent together with the thymus from the third pharyngeal pouch, are relatively frequently (up to 6.3\%) located in the anterior mediastinum.[90]

\section{Parathyroid ${ }^{18} \mathrm{~F}$-fluorocholine PET/CT beyond primary hyperparathyroidism}

Except for imaging in primary hyperparathyroidism, ${ }^{18} \mathrm{~F}-\mathrm{FCH}$ PET/CT can also be used in other parathyroid related abnormalities, such as secondary hyperparathyroidism and tertiary hyperparathyroidism. In secondary hyperparathyroidism, hypersecretion of PTH is stimulated by an external cause, such as impaired intestinal calcium absorption, vitamin D deficiency or renal failure. In renal failure, the most common cause, phosphate retention leads to hypocalcemia and in response, to increased PTH secretion. Continuous stimulation of the parathyroid glands can lead to hyperplasia, which subsequently can lead to autonomous PTH secretion, i.e. tertiary hyperparathyroidism. This condition is usually detected when patients with secondary hyperparathyroidism continue to have elevated PTH levels after treatment of the underlying cause, because the hypertrophied parathyroid tissue fails to resolute and continues to autonomously secrete PTH.[91] This 
most often occurs after kidney transplantation but can also occur with other forms of secondary hyperparathyroidism. Regardless of advances in the medical management in secondary and tertiary hyperparathyroidism, surgery (subtotal parathyroidectomy or total parathyroidectomy with parathyroid autotransplantation) remains a valuable treatment option, especially in tertiary hyperparathyroidism. However, parathyroid glands in these disorders are often small and therefore less likely to be detected before or during surgery. Moreover, as mentioned above, usually multiple parathyroid glands are affected. In order to perform successful surgery, all parathyroid glands have to be identified, including ectopic and supernumerary glands. Although preoperative imaging is not routinely recommended in these cases, imaging can be used to identify all glands and to guide the surgeon. Especially in recurrent disease preoperative imaging can be of added value.[92,93] It has also been suggested that imaging could help to choose the most appropriate gland to be partially preserved when performing a subtotal parathyroidectomy.[94] ${ }^{99 m}$ Tc-sestamibi scintigraphy has been shown to have a sensitivity of only $58 \%$ to detect hyperfunctioning parathyroid glands in secondary and tertiary hyperparathyroidism.[16] Some ${ }^{18} \mathrm{~F}-\mathrm{FCH}$ PET/CT studies investigated mixed cohorts consisting of both patients with primary hyperparathyroidism as well as secondary or tertiary hyperparathyroidism (Table 1); however, without separate analysis of scan performance for the different patient groups. Two recent studies were performed specifically in patients with secondary and tertiary hyperparathyroidism, all due to renal failure.[42,48] In both studies ${ }^{18} \mathrm{~F}-\mathrm{FCH}$ PET/CT was compared with both US and ${ }^{99 m T C-s e s t a m i b i ~ S P E C T / C T, ~ a n d ~ a l l ~ i n c l u d e d ~ p a t i e n t s ~ u n d e r w e n t ~ s u r g e r y . ~ X u e ~ e t ~}$ al. [42] demonstrated a lesion-based sensitivity of $84 \%$ for ${ }^{18} \mathrm{~F}-\mathrm{FCH}$ PET/CT against $63 \%$ and $62 \%$ for US and SPECT/CT, respectively. Findings in the study by Chen et al.[48] were comparable, with lesion-based sensitivity of $86 \%, 55 \%$ and $62 \%$ for ${ }^{18}$ F-FCH PET/ $\mathrm{CT}$, US and SPECT/CT, respectively. Overall, ${ }^{18} \mathrm{~F}-\mathrm{FCH}$ PET/CT yielded favorable detection rates in the abovementioned studies and may be the imaging modality of choice when preoperative imaging is preferred.

${ }^{18} \mathrm{~F}-\mathrm{FCH}$ PET/CT has also been used to detect hyperfunctioning parathyroid glands in familial hyperparathyroidism, such as multiple endocrine neoplasia (MEN) syndromes type 1 and 2a in which hyperparathyroidism is very common. In MEN1, hyperparathyroidism is the most common manifestation with a prevalence of around $90 \%$. Because hyperparathyroidism in MEN1 is often multiglandular and has high recurrence rates, the preferred surgical technique is bilateral neck exploration. Therefore, preoperative imaging is not routinely recommended but is usually reserved for persistent or recurrent disease.[95,96] Detection of hyperfunctioning parathyroid glands in MEN1 patients using ${ }^{18} \mathrm{~F}-\mathrm{FCH}$ PET/CT has recently been studied by Gauthé et 
al. who reported a higher sensitivity of ${ }^{18} \mathrm{~F}-\mathrm{FCH}$ PET/CT compared with conventional

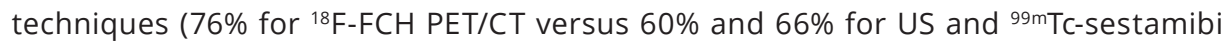
SPECT/CT, respectively).[51] In MEN2a, the prevalence of hyperparathyroidism is lower (20-30\%). Contrary to MEN1, less MEN2a patients present with multiglandular disease and minimal invasive parathyroidectomy is often sufficient with low recurrence rates. Therefore, in MEN2a preoperative imaging to localize the hyperfunctioning parathyroid glands is recommended.[96]

Brown tumors, also known as osteitis fibrosa cystica, are a manifestation of hyperparathyroidism and present as purely lytic skeletal lesions resulting from rapid osteoclastic turnover of the bone. These lesions have occasionally been visualized with ${ }^{11} \mathrm{C}-\mathrm{CH}$ and ${ }^{18} \mathrm{~F}-\mathrm{FCH}$ PET/CT, probably also due to increased cell membrane turnover and/or upregulation of choline kinase.[97,98] Possibly, ${ }^{18} \mathrm{~F}-\mathrm{FCH}$ PET/CT has the potential to differentiate with other lytic bone lesions and to screen the skeleton for additional localizations. ${ }^{18} \mathrm{~F}$-FCH PET/CT was also shown to be able to detect renal osteodystrophy associated with tertiary hyperparathyroidism, as was described in this thesis' intermezzo and an additional case-report.[99] Furthermore, increased uptake of ${ }^{18} \mathrm{~F}-\mathrm{FCH}$ has been demonstrated in parathyroid carcinoma, a rare malignant tumor and a rare cause of hyperparathyroidism. In patients with parathyroid carcinoma, imaging with ${ }^{18} \mathrm{~F}-\mathrm{FCH}$ $\mathrm{PET} / \mathrm{CT}$ could be feasible to detect metastases or local recurrence of the disease after resection.[100,101]

All in all, although relatively little evidence is presently available, ${ }^{18} \mathrm{~F}-\mathrm{FCH}$ PET/CT has been demonstrated to be of additional value in parathyroid diseases other than primary hyperparathyroidism, such as secondary/tertiary hyperparathyroidism, familial hyperparathyroidism syndromes, brown tumors and parathyroid carcinoma.

\section{Scientific methods}

The diagnosis of primary hyperparathyroidism is biochemical, characterized by persistent elevation of total serum calcium levels with corresponding elevated or inappropriately normal PTH levels.[102] In general, parathyroid imaging is only advised to localize hyperfunctioning parathyroid glands preoperatively in biochemically confirmed primary hyperparathyroidism and is not recommended for diagnostic purposes. However, clinical diagnosis is not always clear; serum calcium and PTH can fluctuate, and although guidelines mention that repeated measurements of calcium are required it is not clear how many measurements are necessary and for how long hypercalcemia must be present. Additionally, laboratory tests can under- or overestimate PTH levels 
and the role of vitamin $D$ repletion in patients with low levels of vitamin $D$ associated with hyperparathyroidism needs further evaluation.[103] There is no data on the additional value of ${ }^{18} \mathrm{~F}-\mathrm{FCH}$ PET/CT for diagnosis of primary hyperparathyroidism when there is clinical doubt, but it is reasonable to believe that imaging can provide guidance in those cases; a positive scan result will underpin the clinical diagnosis, while the diagnosis should be reconsidered when ${ }^{18} \mathrm{~F}-\mathrm{FCH}$ PET/CT is negative.

In studies on performance of parathyroid imaging, scan findings have to be correlated with a reference standard. Preferably, each suspicious gland should be surgically resected and histopathologically examined to confirm that the active parathyroid gland on the scan is actually a parathyroid adenoma or a hyperplastic gland, and therefore represents a true-positive result. Acquiring histology by biopsy is not recommended because it can complicate subsequent surgery by inducing inflammation and fibrosis. $[102,104]$ To exclude existence of false-negative parathyroid glands, the patient has to be cured after parathyroidectomy, which means no hyperfunctioning parathyroid glands were missed. If in a cohort also non-cured patients are present (i.e. the surgeon could not locate and remove all the diseased glands), the number of false-negative lesions cannot be determined and sensitivity and negative predictive value cannot be calculated. Because nonsuspicious parathyroid glands are not routinely examined, especially in possible ectopic locations, the number of true-negatives cannot be established and therefore negative predictive value, specificity and accuracy cannot reliably be calculated. Moreover, the use of pre- and post-test probability is complicated in parathyroid imaging. First, because theoretically only patients with definite primary hyperparathyroidism are scanned (100\% pre-test probability), although this is not always the case in clinical practice as mentioned above. Secondly, the "likelihood-ratio", commonly used to calculate post-test probability, cannot be determined reliably. The likelihood-ratio is the ratio between the chance of a positive test result in patients with the disease (sensitivity) and the chance of a positive test result in patients without the disease (1-specificity); however, as discussed above, specificity is generally unknown in parathyroid imaging. The use of positive predictive value (true-positives divided by the sum of true- and false-positives) seems most reliable to estimate post-test probability when pre-test probability is known.

In the cohort study presented in this thesis (chapter 5) a total of 271 patients were included. The large cohort size, compared with other available studies, in one of the main strengths. We used histopathological examination of surgically excised glands in combination with intra- and post-operative PTH measurements as the reference standard. However, not every patient in our cohort underwent surgery and detection rate 
could only be calculated for a subgroup of 139 patients, which is a limitation. A possible explanation for the relatively high rate of non-operated patients could be that ${ }^{18} \mathrm{~F}-\mathrm{FCH}$ $\mathrm{PET} / \mathrm{CT}$ is performed rather early in the diagnostic process in our institution. Potential other reasons why patients not underwent surgery were: contraindications for surgery, patients who refused surgery or postponement of the procedure because of other medical conditions. Patients without visualization of one or more hyperfunctioning glands on ${ }^{18} \mathrm{~F}$-FCH PET/CT probably had a higher change to receive pharmacological treatment or not receiving active treatment at all, rather than undergo parathyroidectomy. Therefore, it is likely that the reported detection rate is an overestimation. No reference standard was available for 132 patients in the cohort, of which 71 had a positive scan and 61 a negative scan. Hypothetically, if all excluded scans were assumed to be correct (71 true-positives and 61 true-negatives), the detection rate would be $97 \%$. If all those scans were assumed to be wrong (71 false-positives and 61 false-negatives), the detection rate would be $66 \%$ compared to a detection rate of $96 \%$ with only the operated patients taken into account. Besides decrease of intraoperatively measured PTH, also absence of relapse in the following months is used to define successful surgery. In our cohort study the follow-up was 3-43 months, although some guidelines define cure as the reestablishment of normal calcium homeostasis lasting a minimum of 6 months.[102] As mentioned above, confirmation of cure is needed to exclude false-negative results and to adequately calculate detection rate. In studies concerning scan acquisition protocols such as those presented in chapter $\mathbf{3}$ and chapter $\mathbf{4}$ of this thesis, exclusion of patients with negative scan results does not lead to bias in the way it does in studies on scan performance. Only scans with histopathologically proven parathyroid adenomas or hyperplastic glands were analyzed in the two mentioned studies, which only strengthens the conclusions on the ${ }^{18} \mathrm{~F}-\mathrm{FCH}$ uptake patterns in those glands.

The studies in this thesis have a retrospective design. Such studies usually have the limitation that not all desired data can be collected, because some measurements were not performed in all patients. For example in our own cohort study, a 24-h urinary calcium or vitamin D level was not available for every patient. Additionally, there is more risk of selection bias, which has been minimized in our cohort study by consecutively including patients following strict inclusion criteria. Moreover, it is crucial to interpret the index test $\left({ }^{18} \mathrm{~F}-\mathrm{FCH}\right.$ PET/CT) independently from the reference standard (surgery and pathology results). To achieve this, scan results were entered into database directly after reading of the scan. Furthermore, equivocal scans were discussed in a consensus meeting blinded from follow-up data. As mentioned before, one of the main problems concerning our own cohort study, along with a substantial number of other studies on ${ }^{18} \mathrm{~F}-\mathrm{FCH}$ PET/CT parathyroid localization, was the lack of an available reference standard 
for every patient. Although this problem can also present with a prospective study, for example due to unforeseen contra-indications to surgery or loss-to-follow-up, it is more feasible to exclude patients without a clear indication for surgery in a prospective design. With a prospective study design it is possible to strictly protocolize the patient work-up and treatment strategy which can be regulated with quality checks during the study. Another advantage of a prospective study is the possibility to directly compare two or more different imaging modalities. On the other hand, a retrospective cohort study like the one presented in this thesis, potentially reflects clinical practice better than a prospective cohort: in a retrospective study the clinical work-up is less strictly protocolized and more dependent on personal decisions of the treating physicians. Still, our study had a single-center design and translation of the results to other centers should be done with caution.

\section{Future developments and research opportunities}

Several developments in parathyroid imaging with ${ }^{18} \mathrm{~F}-\mathrm{FCH}$ PET/CT could lead to a further increase of accuracy in the future. One of the most promising developments is the ongoing transition of analog PET scanners (using conventional photomultiplier tubes for photon-to-electron conversion) to digital PET scanners (in which the tubes are replaced by high-performance solid-state detectors and every detector is coupled to its own scintillation crystal). Digital PET systems have higher sensitivity and higher spatial as well as temporal resolution, resulting in better image quality, diagnostic confidence and accuracy.[105,106] Especially for small lesions, such as parathyroid adenomas, digital PET/CT demonstrates higher detection rates. In a recent study by López-Mora et al.[46], 33 patients with primary hyperparathyroidism were scanned with both an analog and a digital PET/CT system. Hyperfunctioning parathyroid glands were detected in 30/33 patients with the digital system whereas those glands were detected with the analog system in only 22/33 patients. Each of the lesions that was solely detected by the digital PET/CT system was $<10 \mathrm{~mm}$ at surgical resection. Although digital PET/CT scanners still are relatively expensive, it is expected that in the coming years these systems will become more available. As mentioned before, it is possible to combine ${ }^{18} \mathrm{~F}-\mathrm{FCH}$ PET with MRI on dedicated PET/MRI scanners. The advantages are mainly the combination of the high detection rate of ${ }^{18} \mathrm{~F}-\mathrm{FCH}$ PET with the superior soft-tissue resolution of MRI together with the reduction of radiation exposure. Moreover, first results of clinical use in hyperparathyroidism are favorable.[29,33,40] Currently, only few PET/MRI systems are available, purchase costs are high and clinical applicability in general has not been clearly delineated. In the near future, these systems are probably limited to academic centers and will be mainly used in research settings. 
Several more questions remain unanswered at the time of writing this thesis. Along with ${ }^{18} \mathrm{~F}-\mathrm{FCH} \mathrm{PET} / \mathrm{CT}$, the currently most extensive studied imaging modality to visualize parathyroid glands is $4 \mathrm{D}-\mathrm{CT}$. Although available studies comparing these modalities generally show favorable results for ${ }^{18} \mathrm{~F}-\mathrm{FCH}$ PET as was mentioned earlier, there is a need for prospectively designed studies with larger populations in which each imaging modality is reviewed separately and preferably findings are histopathologically confirmed for every patient. This could clarify which modality is more accurate and if a "one-stop-shop" ${ }^{18} \mathrm{~F}-\mathrm{FCH}$ PET/4D-CT is of additional value, considering the tradeoff between more detailed anatomical information for better surgical guidance and higher radiation exposure from multi-phase CT. Usually it is not advised to discontinue

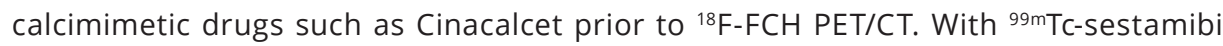
scintigraphy, however, it has been demonstrated that hyperplastic parathyroid glands can show decreased uptake after treatment with calcimimetics.[107] Studies on the effect of calcimimetics on ${ }^{18} \mathrm{~F}-\mathrm{FCH}$ PET/CT performance could clarify if these medications can indeed be used without impairing the detection of parathyroid glands. Furthermore, those studies could demonstrate the ability of ${ }^{18} \mathrm{~F}-\mathrm{FCH}$ PET/CT to monitor treatment response. Future research could also further clarify the uptake mechanisms of ${ }^{18} \mathrm{~F}-\mathrm{FCH}$ in hyperfunctioning parathyroid glands and may correlate the level of ${ }^{18} \mathrm{~F}-\mathrm{FCH}$ uptake with the different histopathological subtypes of parathyroid lesions, since expanding our basic knowledge of this scanning technique could possibly lead to broader applications. Finally, it is important to perform cost-effectiveness analyses on the use of ${ }^{18} \mathrm{~F}-\mathrm{FCH}$ $\mathrm{PET/CT}$. Earlier it has been demonstrated that advanced imaging techniques such as SPECT, SPECT/CT and 4D-CT are associated with cost-savings due to shortening surgical times and obviating the need for more costly bilateral neck explorations.[108,109] No comparable analyses are currently available for ${ }^{18} \mathrm{~F}-\mathrm{FCH}$ PET/CT but such assessment would contribute to justify its use for parathyroid imaging, especially as a first-line imaging modality.

In conclusion, although certain aspects of ${ }^{18} \mathrm{~F}-\mathrm{FCH}$ PET/CT parathyroid imaging may be further elucidated, the technique consistently yields excellent results as has been described in the scientific literature and elaborated on in this thesis. It has been demonstrated to be superior compared to ${ }^{99 \mathrm{mTC}} \mathrm{C}$-sestamibi SPECT/CT and also compared with newer techniques such as 4D-CT and MRI. ${ }^{18} \mathrm{~F}-\mathrm{FCH}$ PET/CT not only seems suitable in case conventional imaging methods are inconclusive, but can also be effectively deployed as a first-line imaging modality to detect hyperfunctioning parathyroid glands, although cost-effectiveness analyses are warranted. Moreover, future research will be relevant for further optimization of scan acquisition protocols and to evaluate the additional value of novel hybrid scanning techniques such as PET/4D-CT or PET/MRI. 


\section{References}

1. Vallabhajosula S. ${ }^{18} \mathrm{~F}$-labeled positron emission tomographic radiopharmaceuticals in oncology: an overview of radiochemistry and mechanisms of tumor localization. Semin Nucl Med 2007;37:400-419.

2. Glunde K, Penet MF, Jiang L, Jacobs MA, Bhujwalla ZM. Choline metabolism-based molecular diagnosis of cancer: an update. Expert Rev Mol Diagn 2015;15:735-747.

3. Hara T, Kosaka N, Kishi H. PET imaging of prostate cancer using carbon-11-choline. J Nucl Med 1998;39:990-995.

4. Conti M, Eriksson L. Physics of pure and non-pure positron emitters for PET: a review and a discussion. EJNMMI Phys 2016;3:8.

5. DeGrado TR, Reiman RE, Price DT, Wang S, Coleman RE. Pharmacokinetics and radiation dosimetry of ${ }^{18} \mathrm{~F}$-fluorocholine. J Nucl Med 2002;43:92-96.

6. Hara T, Kosaka N, Kishi H. Development of ${ }^{18} \mathrm{~F}$-fluoroethylcholine for cancer imaging with PET: synthesis, biochemistry, and prostate cancer imaging. J Nucl Med 2002;43:187-199.

7. DeGrado TR, Baldwin SW, Wang S, Orr MD, Liao RP, Friedman HS, et al. Synthesis and evaluation of ${ }^{18}$ F-labeled choline analogs as oncologic PET tracers. J Nucl Med 2001;42:1805-1814.

8. Evangelista L, Cervino AR, Guttilla A, Zattoni F, Cuccurullo V, Mansi L. ${ }^{18}$ F-fluoromethylcholine or ${ }^{18} \mathrm{~F}$-fluoroethylcholine pet for prostate cancer imaging: which is better? A literature revision. Nucl Med Biol 2015;42:340-348.

9. Quak E, Lheureux S, Reznik Y, Bardet S, Aide N. F18-choline, a novel PET tracer for parathyroid adenoma? J Clin Endocrinol Metab 2013;98:3111-3112.

10. Nakagami K, Uchida T, Ohwada S, Koibuchi Y, Suda Y, Sekine T, et al. Increased choline kinase activity and elevated phosphocholine levels in human colon cancer. Jpn J Cancer Res 1999;90:419-424.

11. Battini S, Imperiale A, Taieb D, Elbayed K, Cicek AE, Sebag F, et al. High-resolution magic angle spinning ${ }^{1} \mathrm{H}$ nuclear magnetic resonance spectroscopy metabolomics of hyperfunctioning parathyroid glands. Surgery 2016;160:384-394.

12. Piccardo A, Trimboli P, Rutigliani M, Puntoni M, Foppiani L, Bacigalupo $L$, et al. Additional value of integrated ${ }^{18} \mathrm{~F}$-choline PET/4D contrast-enhanced CT in the localization of hyperfunctioning parathyroid glands and correlation with molecular profile. Eur J Nucl Med Mol Imaging 2018;46:766-775.

13. Treglia G, Piccardo A, Imperiale A, Strobel K, Kaufmann PA, Prior JO, et al. Diagnostic performance of choline PET for detection of hyperfunctioning parathyroid glands in hyperparathyroidism: a systematic review and meta-analysis. Eur J Nucl Med Mol Imaging 2018;46:751-765.

14. Kim SJ, Lee SW, Jeong SY, Pak K, Kim K. Diagnostic performance of F-18 fluorocholine PET/CT for parathyroid localization in hyperparathyroidism: a systematic review and meta-analysis. Horm Cancer 2018;9:440-447.

15. Boccalatte LA, Higuera F, Gomez NL, de la Torre AY, Mazzaro EL, Galich AM, et al. Usefulness of ${ }^{18} \mathrm{~F}$-fluorocholine positron emission tomography-computed tomography in locating lesions in hyperparathyroidism: a Systematic review. JAMA Otolaryngol Head Neck Surg 2019;145:743-750.

16. Caldarella C, Treglia G, Pontecorvi A, Giordano A. Diagnostic performance of planar scintigraphy

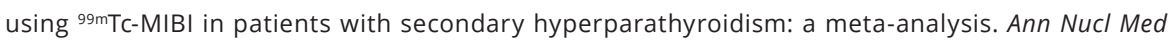
2012;26:794-803.

17. Christakis I, Khan S, Sadler GP, Gleeson FV, Bradley KM, Mihai R. ${ }^{18}$ F-Fluorocholine PET/CT scanning with arterial phase-enhanced CT is useful for persistent/recurrent primary hyperparathyroidism: first UK case series results. Ann R Coll Surg Eng/ 2019;101:501-507. 
18. Evangelista L, Ravelli I, Magnani F, lacobone M, Giraudo C, Camozzi V, et al. ${ }^{18} \mathrm{~F}$-choline PET/CT and PET/ MRI in primary and recurrent hyperparathyroidism: a systematic review of the literature. Ann NuCl Med 2020;34:601-619.

19. Lezaic L, Rep S, Sever MJ, Kocjan T, Hocevar M, Fettich J. ${ }^{18}$ F-Fluorocholine PET/CT for localization of hyperfunctioning parathyroid tissue in primary hyperparathyroidism: a pilot study. Eur J Nucl Med Mol Imaging 2014;41:2083-2089.

20. Michaud L, Burgess A, Huchet V, Lefevre M, Tassart M, Ohnona J, et al. Is ${ }^{18} \mathrm{~F}$-fluorocholine-positron emission tomography/computerized tomography a new imaging tool for detecting hyperfunctioning parathyroid glands in primary or secondary hyperparathyroidism? J Clin Endocrinol Metab 2014;99:45314536.

21. Orevi M, Freedman N, Mishani E, Bocher M, Jacobson O, Krausz Y. Localization of parathyroid adenoma by ${ }^{11} \mathrm{C}$-choline PET/CT: preliminary results. Clin Nucl Med 2014;39:1033-1038.

22. Kluijfhout WP, Vorselaars WM, Vriens MR, Borel Rinkes IH, Valk GD, de Keizer B. Enabling minimal invasive parathyroidectomy for patients with primary hyperparathyroidism using Tc-99m-sestamibi SPECT-CT, ultrasound and first results of ${ }^{18} \mathrm{~F}$-fluorocholine PET-CT. Eur J Radio/ 2015;84:1745-1751.

23. Michaud L, Balogova S, Burgess A, Ohnona J, Huchet V, Kerrou K, et al. A pilot comparison of ${ }^{18} \mathrm{~F}$-fluorocholine PET/CT, ultrasonography and ${ }^{123} /{ }^{99} \mathrm{~m} T \mathrm{~T}$-sestaMIBI dual-phase dual-isotope scintigraphy in the preoperative localization of hyperfunctioning parathyroid glands in primary or secondary hyperparathyroidism: influence of thyroid anomalies. Medicine (Baltimore) 2015;94:e1701.

24. Rep S, Lezaic L, Kocjan T, Pfeifer M, Sever MJ, Simoncic U, et al. Optimal scan time for evaluation of parathyroid adenoma with ${ }^{18}$ F-fluorocholine PET/CT. Radiol Oncol 2015;49:327-333.

25. Kluijfhout WP, Vorselaars WM, van den Berk SA, Vriens MR, Borel Rinkes IH, Valk GD, et al. Fluorine-18 fluorocholine PET-CT localizes hyperparathyroidism in patients with inconclusive conventional imaging: a multicenter study from the Netherlands. Nucl Med Commun 2016;37:1246-1252.

26. Hocevar M, Lezaic L, Rep S, Zaletel K, Kocjan T, Sever MJ, et al. Focused parathyroidectomy without intraoperative parathormone testing is safe after pre-operative localization with ${ }^{18} \mathrm{~F}$-fluorocholine PET/CT. Eur J Surg Oncol 2017;43:133-137.

27. Taywade SK, Damle NA, Behera A, Devasenathipathy K, Bal C, Tripathi M, et al. Comparison of ${ }^{18} \mathrm{~F}$-fluorocholine positron emission tomography/computed tomography and four-dimensional computed tomography in the preoperative localization of parathyroid adenomas-initial results. Indian J Endocrinol Metab 2017;21:399-403.

28. Thanseer N, Bhadada SK, Sood A, Mittal BR, Behera A, Gorla AKR, et al. comparative effectiveness of ultrasonography, ${ }^{99 \mathrm{mTC}} \mathrm{T}-\mathrm{sestamibi}$, and ${ }^{18} \mathrm{~F}$-fluorocholine PET/CT in detecting parathyroid adenomas in patients With primary hyperparathyroidism. Clin Nucl Med 2017;42:e491-e497.

29. Kluijfhout WP, Pasternak JD, Gosnell JE, Shen WT, Duh QY, Vriens MR, et al. ${ }^{18} \mathrm{~F}$ fluorocholine PET/ MR imaging in patients with primary hyperparathyroidism and inconclusive conventional imaging: a prospective pilot study. Radiology 2017;284:460-467.

30. Quak E, Blanchard D, Houdu B, Le Roux Y, Ciappuccini R, Lireux B, et al. F18-choline PET/CT guided surgery in primary hyperparathyroidism when ultrasound and MIBI SPECT/CT are negative or inconclusive: the APACH1 study. Eur J Nucl Med Mol Imaging 2017;45:658-666.

31. Rep S, Hocevar M, Vaupotic J, Zdesar U, Zaletel K, Lezaic L. ${ }^{18} \mathrm{~F}$-choline PET/CT for parathyroid scintigraphy: significantly lower radiation exposure of patients in comparison to conventional nuclear medicine imaging approaches. J Radiol Prot 2018;38:343-356.

32. Fischli S, Suter-Widmer I, Nguyen BT, Muller W, Metzger J, Strobel K, et al. The significance of ${ }^{18} \mathrm{~F}$-fluorocholine-PET/CT as localizing imaging technique in patients with primary hyperparathyroidism and negative conventional imaging. Front Endocrinol (Lausanne) 2018;8:380. 
33. Huber GF, Hullner M, Schmid C, Brunner A, Sah B, Vetter D, et al. Benefit of ${ }^{18} \mathrm{~F}$-fluorocholine PET imaging in parathyroid surgery. Eur Radiol 2018;28:2700-2707.

34. Beheshti M, Hehenwarter L, Paymani Z, Rendl G, Imamovic L, Rettenbacher R, et al. ${ }^{18}$ F-Fluorocholine $\mathrm{PET/CT}$ in the assessment of primary hyperparathyroidism compared with ${ }^{99 \mathrm{~m} T \mathrm{TC}-\mathrm{MIBI} \text { or }}{ }^{99 \mathrm{~m} \mathrm{TC}-}$ tetrofosmin SPECT/CT: a prospective dual-centre study in 100 patients. Eur J Nucl Med Mol Imaging 2018;45:1762-1771.

35. Grimaldi S, Young J, Kamenicky P, Hartl D, Terroir M, Leboulleux S, et al. Challenging pre-surgical localization of hyperfunctioning parathyroid glands in primary hyperparathyroidism: the added value of ${ }^{18} \mathrm{~F}$-Fluorocholine PET/CT. EurJ Nucl Med Mol Imaging 2018;45:1772-1780.

36. Bossert I, Chytiris S, Hodolic M, Croce L, Mansi L, Chiovato L, et al. PETC/CT with ${ }^{18} \mathrm{~F}$-Choline localizes hyperfunctioning parathyroid adenomas equally well in normocalcemic hyperparathyroidism as in overt hyperparathyroidism. J Endocrinol Invest 2018;42:419-426.

37. Araz M, Soydal C, Ozkan E, Kir MK, Ibis E, Gullu S, et al. The efficacy of fluorine-18-choline PET/CT in

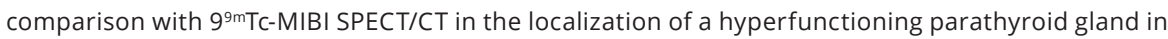
primary hyperparathyroidism. Nucl Med Commun 2018;39:989-994.

38. Zajickova K, Zogala D, Kubinyi J. Parathyroid imaging by ${ }^{18} \mathrm{~F}$-fluorocholine PET/CT in patients with primary hyperparathyroidism and inconclusive conventional methods: clinico-pathological correlations. Physiol Res 2018;67:S551-S557.

39. Amadou C, Bera G, Ezziane M, Chami L, Delbot T, Rouxel A, et al. ${ }^{18} \mathrm{~F}$-fluorocholine PET/CT and parathyroid 4D computed tomography for primary hyperparathyroidism: the challenge of reoperative patients. World J Surg 2019;43:1232-1242.

40. Khafif A, Masalha M, Landsberg R, Domachevsky L, Bernstine H, Groshar D, et al. The role of F18fluorocholine positron emission tomography/magnetic resonance imaging in localizing parathyroid adenomas. Eur Arch Otorhinolaryngol 2019;276:1509-1516.

41. Broos WAM, Wondergem M, Knol RJJ, van der Zant FM. Parathyroid imaging with ${ }^{18}$ F-fluorocholine $\mathrm{PET} / \mathrm{CT}$ as a first-line imaging modality in primary hyperparathyroidism: a retrospective cohort study. EJNMMI Res 2019;9:72.

42. Xue Y, Li W, Xia Z, Lei C, Cao Y, Wang Z, et al. The role of ${ }^{18} \mathrm{~F}-\mathrm{FCH}$ PET/CT in patients with uremic hyperparathyroidism compared with ${ }^{99 \mathrm{mTC}}$-sestaMIBI SPECT/CT and ultrasonography. EJNMMI Res 2019;9:118.

43. Cuderman A, Senica K, Rep S, Hocevar M, Kocjan T, Jensterle Sever M, et al. ${ }^{18} \mathrm{~F}$-Fluorocholine PET/ CT in primary hyperparathyroidism: superior diagnostic performance to conventional scintigraphic imaging for localization of hyperfunctioning parathyroid glands. J Nucl Med 2020;61:577-583.

44. Liu Y, Dang Y, Huo L, Hu Y, Wang O, Liu H, et al. Preoperative localisation of adenomas in primary hyperparathyroidism: the value of ${ }^{11} \mathrm{C}$-choline PET/CT in patients with negative or discordant ultrasonography and 99mTc-Sesta-MIBI-SPECT/CT. J Nucl Med 2020;61:584-589.

45. Morland D, Lalire P, Deguelte S, Zalzali M, Richard C, Dejust S, et al. Added value of ${ }^{18} \mathrm{~F}$-fluorocholine positron emission tomography-computed tomography in presurgical localization of hyperfunctioning parathyroid glands after dual tracer subtraction scintigraphy failure: A retrospective study of 47 patients. Medicine (Baltimore) 2020;99:e18681.

46. Lopez-Mora DA, Sizova M, Estorch M, Flotats A, Camacho V, Fernandez A, et al. Superior performance of ${ }^{18} \mathrm{~F}$-fluorocholine digital PET/CT in the detection of parathyroid adenomas. Eur J Nucl Med Mol Imaging 2020; 47:572-578.

47. Uslu-Besli L, Sonmezoglu K, Teksoz S, Akgun E, Karayel E, Pehlivanoglu H, et al. Performance of F-18 fluorocholine PET/CT for detection of hyperfunctioning parathyroid tissue in patients with elevated parathyroid hormone levels and negative or discrepant results in conventional imaging. Korean J Radiol 2020;21:236-247. 
48. Chen YH, Chen HT, Lee MC, Liu SH, Wang LY, Lue KH, et al. Preoperative F-18 fluorocholine PET/CT for the detection of hyperfunctioning parathyroid glands in patients with secondary or tertiary hyperparathyroidism: comparison with Tc-99m sestamibi scan and neck ultrasound. Ann Nucl Med 2020;34:527-537.

49. Boccalatte LA, Gomez NL, Musumeci M, Galich AM, Collaud C, Figari MF. ${ }^{18} \mathrm{~F}$-choline PET/4D CT in hyperparathyroidism: correlation between biochemical data and study parameters. Rev Esp Med NuCl Imagen Mol 2020;39:273-278.

50. Pretet V, Rotania M, Helali M, Ignat M, Vix M, Imperiale A. ${ }^{18} \mathrm{~F}$-fluorocholine PET and multiphase CT integrated in dual modality PET/4D-CT for preoperative evaluation of primary hyperparathyroidism. J Clin Med 2020;9:2005.

51. Gauthe M, Dierick-Gallet A, Delbot T, Bricaire L, Bertherat J, North MO, et al. ${ }^{18} \mathrm{~F}$-fluorocholine PET/CT in MEN1 patients with primary hyperparathyroidism. World J Surg 2020;44:3761-3769.

52. Broos WAM, van der Zant FM, Knol RJJ, Wondergem M. Choline PET/CT in parathyroid imaging: a systematic review. Nucl Med Commun 2019;40:96-105.

53. Wong KK, Fig LM, Gross MD, Dwamena BA. Parathyroid adenoma localization with ${ }^{99 m}$ Tc-sestamibi SPECT/CT: a meta-analysis. Nucl Med Commun 2015;36:363-375.

54. Patel CN, Salahudeen HM, Lansdown M, Scarsbrook AF. Clinical utility of ultrasound and ${ }^{99 m} T C$ sestamibi SPECT/CT for preoperative localization of parathyroid adenoma in patients with primary hyperparathyroidism. Clin Radiol 2010;65:278-287.

55. Noda S, Onoda N, Kashiwagi S, Kawajiri H, Takashima T, Ishikawa T, et al. Strategy of operative treatment of hyperparathyroidism using US scan and ${ }^{99 m}$ TC-MIBI SPECT/CT. Endocr J 2014;61:225-230.

56. Erbil Y, Barbaros U, Yanik BT, Salmaslioglu A, Tunaci M, Adalet I, et al. Impact of gland morphology and concomitant thyroid nodules on preoperative localization of parathyroid adenomas. Laryngoscope 2006;116:580-585.

57. Parra Ramírez P, Santiago Hernando A, Barquiel Alcalá B, Martín Rojas-Marcos P, Lisbona Catalán A, Álvarez Escolá C. Potential utility of contrast-enhanced ultrasound in the preoperative evaluation of primary hyperparathyroidism. J Ultrasound Med 2019;38:2565-2571.

58. Stangierski A, Wolinski K, Ruchala M. Shear wave elastography in the diagnostics of parathyroid adenomas-new application of the method. Endocrine 2018;60:240-245.

59. Kluijfhout WP, Pasternak JD, Beninato T, Drake FT, Gosnell JE, Shen WT, et al. Diagnostic performance of computed tomography for parathyroid adenoma localization; a systematic review and meta-analysis. Eur J Radiol 2017;88:117-128.

60. Yeh R, Tay YD, Tabacco G, Dercle L, Kuo JH, Bandeira L, et al. Diagnostic performance of 4D CT and sestamibi SPECT/CT in localizing parathyroid adenomas in primary hyperparathyroidism. Radiology 2019;291:469-476.

61. Bahl M. Preoperative parathyroid imaging: trends in utilization and comparative accuracy of sonography, scintigraphy, and 4-dimensional computed tomography. J Comput Assist Tomogr 2019;43:264-268.

62. Becker JL, Patel V, Johnson KJ, Guerrero M, Klein RR, Ranvier GF, et al. 4D-dynamic contrast-enhanced MRI for preoperative localization in patients with primary hyperparathyroidism. AJNR Am J Neuroradiol 2020;41:522-528.

63. Nael K, Hur J, Bauer A, Khan R, Sepahdari A, Inampudi R, et al. Dynamic 4D MRI for characterization of parathyroid adenomas: multiparametric analysis. AJNR Am J Neuroradio/ 2015;36:2147-2152.

64. Kluijfhout WP, Pasternak JD, Drake FT, Beninato T, Gosnell JE, Shen WT, et al. Use of PET tracers for parathyroid localization: a systematic review and meta-analysis. Langenbecks Arch Surg 2016;401:925935. 
65. Neumann DR, Esselstyn CB,Jr, Maclntyre WJ, Chen EQ, Go RT, Kohse LM, et al. Primary hyperparathyroidism: preoperative parathyroid imaging with regional body FDG PET. Radiology 1994;192:509-512.

66. Melon P, Luxen A, Hamoir E, Meurisse M. Fluorine-18-fluorodeoxyglucose positron emission tomography for preoperative parathyroid imaging in primary hyperparathyroidism. Eur J Nucl Med 1995;22:556-558.

67. Chicklore S, Schulte KM, Talat N, Hubbard JG, O'Doherty M, Cook GJ. ${ }^{18}$ F-FDG PET rarely provides additional information to ${ }^{11} \mathrm{C}-$ methionine PET imaging in hyperparathyroidism. Clin Nucl Med 2014;39:237-242.

68. Sisson JC, Thompson NW, Ackerman RJ, Wahl RL. Use of 2-[F-18]-fluoro-2-deoxy-D-glucose PET to locate parathyroid adenomas in primary hyperparathyroidism. Radiology 1994;192:280.

69. Krakauer M, Kjaer A, Bennedbaek FN. ${ }^{18} \mathrm{~F}-\mathrm{FET}$-PET in primary hyperparathyroidism: a pilot study. Diagnostics (Basel) 2016;6:30.

70. Lange-Nolde A, Zajic T, Slawik M, Brink I, Reincke M, Moser E, et al. PET with ${ }^{18} \mathrm{~F}$-DOPA in the imaging of parathyroid adenoma in patients with primary hyperparathyroidism. A pilot study. Nuklearmedizin 2006;45:193-196.

71. Cheung K, Wang TS, Farrokhyar F, Roman SA, Sosa JA. A meta-analysis of preoperative localization techniques for patients with primary hyperparathyroidism. Ann Surg Oncol 2012;19:577-583.

72. Roef MJ, van der Poel H, van der Laken CJ, Vogel WV. Colchicine must be stopped before imaging with ${ }^{18} \mathrm{~F}$-methylcholine PET/CT. Nucl Med Commun 2010;31:1075-1077.

73. Greenspan BS, Dillehay G, Intenzo C, Lavely WC, O'Doherty M, Palestro CJ, et al. SNM practice guideline for parathyroid scintigraphy 4.0.J Nucl Med Technol 2012;40:111-118.

74. Hindie E, Ugur O, Fuster D, O'Doherty M, Grassetto G, Urena P, et al. 2009 EANM parathyroid guidelines. EurJ Nucl Med Mol Imaging 2009;36:1201-1216.

75. Hoang JK, Reiman RE, Nguyen GB, Januzis N, Chin BB, Lowry C, et al. Lifetime attributable risk of cancer from radiation exposure during parathyroid imaging: comparison of 4D CT and parathyroid scintigraphy. AJR Am J Roentgenol 2015;204:W579-85.

76. Tolvanen T, Yli-Kerttula T, Ujula T, Autio A, Lehikoinen P, Minn H, et al. Biodistribution and radiation dosimetry of $\left[{ }^{11} \mathrm{C}\right]$ choline: a comparison between rat and human data. Eur J Nucl Med Mol Imaging 2010;37:874-883.

77. ICRP. Radiation dose to patients from radiopharmaceuticals. Addendum 3 to ICRP Publication 53. ICRP Publication 106. Approved by the Commission in October 2007. Ann ICRP 2008;38:1-197.

78. ICRP. Radiation dose to patients from radiopharmaceuticals (addendum 2 to ICRP publication 53). Ann ICRP 1998;28:1-126.

79. Mahajan A, Starker LF, Ghita M, Udelsman R, Brink JA, Carling T. Parathyroid four-dimensional computed tomography: evaluation of radiation dose exposure during preoperative localization of parathyroid tumors in primary hyperparathyroidism. World J Surg 2012;36:1335-1339.

80. Calabria F, Chiaravalloti A, Ciccio C, Gangemi V, Gulla D, Rocca F, et al. PET/CT with ${ }^{18}$ F-choline: Physiological whole bio-distribution in male and female subjects and diagnostic pitfalls on 1000 prostate cancer patients: ${ }^{18} \mathrm{~F}$-choline PET/CT bio-distribution and pitfalls. A southern Italian experience. Nucl Med Biol 2017;51:40-54.

81. How Kit N, Dugue AE, Sevin E, Allouache N, Lesaunier F, Joly F, et al. Pairwise comparison of ${ }^{18} \mathrm{~F}-\mathrm{FDG}$ and ${ }^{18} \mathrm{~F}-\mathrm{FCH}$ PET/CT in prostate cancer patients with rising PSA and known or suspected second malignancy. Nucl Med Commun 2016;37:348-355. 
82. Garcia JR, Ponce A, Canales M, Ayuso J, Moragas M, Soler M. Detection of second tumors in ${ }^{11} \mathrm{C}$-choline $\mathrm{PET/CT}$ studies performed due to biochemical recurrence of prostate cancer. Rev Esp Med Nucl Imagen Mol 2014;33:28-31.

83. Mallick R, Malik J, Yip L, Muthukrishnan A, Carty SE, McCoy KL. Novel findings on SPECT-CT TC-99 sestamibi imaging for primary hyperparathyroidism. J Surg Res 2020;252:216-221.

84. Snider SA, Margison KD, Ghorbani P, LeBlond ND, O'Dwyer C, Nunes JRC, et al. Choline transport links macrophage phospholipid metabolism and inflammation. J Biol Chem 2018;293:11600-11611.

85. Wyss MT, Weber B, Honer M, Spath N, Ametamey SM, Westera G, et al. ${ }^{18} \mathrm{~F}$-choline in experimental soft tissue infection assessed with autoradiography and high-resolution PET. Eur J Nucl Med Mol Imaging 2004;31:312-316.

86. Vellani C, Hodolic M, Chytiris S, Trifiro G, Rubello D, Colletti PM. Early and delayed ${ }^{18}$ F-FCH PET/CT imaging in parathyroid adenomas. Clin Nucl Med 2017;42:143-144.

87. Prabhu M, Kumari G, Damle NA, Arora G, Kumar P, Kumar R, et al. Assessment of the role of early dynamic PET/CT with ${ }^{18} \mathrm{~F}$-fluorocholine in detection of parathyroid lesions in patients with primary hyperparathyroidism. Nucl Med Commun 2018;39:1190-1196.

88. Morland D, Richard C, Godard F, Deguelte S, Delemer B. Temporal uptake patterns of ${ }^{18} \mathrm{~F}$-Fluorocholine among hyperfunctioning parathyroid glands. Clin Nucl Med 2018;43:504-505.

89. Lengelé B, Hamoir M. Anatomy and embryology of the parathyroid glands. Acta Otorhinolaryngol Belg 2001;55:89-93.

90. Lappas D, Noussios G, Anagnostis P, Adamidou F, Chatzigeorgiou A, Skandalakis P. Location, number and morphology of parathyroid glands: results from a large anatomical series. Anat Sci Int 2012;87:160-164.

91. Pitt SC, Sippel RS, Chen H. Secondary and tertiary hyperparathyroidism, state of the art surgical management. Surg Clin North Am 2009;89:1227-1239.

92. Lorenz K, Bartsch DK, Sancho JJ, Guigard S, Triponez F. Surgical management of secondary hyperparathyroidism in chronic kidney disease - a consensus report of the European Society of Endocrine Surgeons. Langenbecks Arch Surg 2015;400:907-927.

93. van der Plas WY, Noltes ME, van Ginhoven TM, Kruijff S. Secondary and tertiary hyperparathyroidism: a narrative review. Scand J Surg 2020;109:271-278.

94. Taieb D, Hindie E, Grassetto G, Colletti PM, Rubello D. Parathyroid scintigraphy: when, how, and why? A concise systematic review. Clin Nucl Med 2012;37:568-574.

95. van Leeuwaarde RS, de Laat JM, Pieterman CRC, Dreijerink K, Vriens MR, Valk GD. The future: medical advances in MEN1 therapeutic approaches and management strategies. Endocr Relat Cancer 2017;24:T179-T193.

96. Twigt BA, Scholten A, Valk GD, Rinkes IH, Vriens MR. Differences between sporadic and MEN related primary hyperparathyroidism; clinical expression, preoperative workup, operative strategy and follow-up. Orphanet J Rare Dis 2013;8:50.

97. Garcia JR, Alvarez Moro FJ, Bassa P, Soler M, Llinares E, Riera E. Brown tumours due to secondary hyperparathyroidism detected by ${ }^{11}$ C-choline PET/CT. Rev Esp Med Nucl Imagen Mol 2016;35:209-210.

98. Zhang-Yin J, Gaujoux S, Delbot T, Gauthe M, Talbot JN. ${ }^{18} \mathrm{~F}$-Fluorocholine PET/CT imaging of brown tumors in a patient with severe primary hyperparathyroidism. Clin Nucl Med 2019;44:971-974.

99. Ferrari C, Lavelli V, Santo G, Frugis MT, Iuele F, Rubini G, et al. ${ }^{18} \mathrm{~F}$-Fluorocholine PET/CT, Tc-99m-MIBI and TC-99m-MDP SPECT/CT in tertiary hyperparathyroidism with renal osteodystrophy. Diagnostics (Basel) 2020;10:E851.

100. Hatzl M, Roper-Kelmayr JC, Fellner FA, Gabriel M. ${ }^{18} \mathrm{~F}$-Fluorocholine, ${ }^{18} \mathrm{~F}$-FDG, and ${ }^{18} \mathrm{~F}$-Fluoroethyl Tyrosine PET/CT in Parathyroid Cancer. Clin Nucl Med 2017;42:448-450. 
101. Deandreis D, Terroir M, Al Ghuzlan A, Berdelou A, Lacroix L, Bidault F, et al. ${ }^{18} \mathrm{~F}$-Fluorocholine PET/CT in parathyroid carcinoma: a new tool for disease staging? Eur J Nucl Med Mol Imaging 2015;42:19411942.

102. Wilhelm SM, Wang TS, Ruan DT, Lee JA, Asa SL, Duh QY, et al. The American Association of Endocrine Surgeons guidelines for definitive management of primary hyperparathyroidism. JAMA Surg 2016;151:959-968.

103. Eastell R, Brandi ML, Costa AG, D'Amour P, Shoback DM, Thakker RV. Diagnosis of asymptomatic primary hyperparathyroidism: proceedings of the Fourth International Workshop. J Clin Endocrinol Metab 2014;99:3570-3579.

104. Norman J, Politz D, Browarsky I. Diagnostic aspiration of parathyroid adenomas causes severe fibrosis complicating surgery and final histologic diagnosis. Thyroid 2007;17:1251-1255.

105. Zhang J, Maniawski P, Knopp MV. Performance evaluation of the next generation solid-state digital photon counting PET/CT system. EJNMMI Res 2018;8:97.

106. Nguyen NC, Vercher-Conejero JL, Sattar A, Miller MA, Maniawski PJ, Jordan DW, et al. Image quality and diagnostic performance of a digital PET prototype in patients with oncologic diseases: initial experience and comparison with analog PET. J Nucl Med 2015;56:1378-1385.

107. Fuster D, Torregrosa JV, Domenech B, Solà O, Martín G, Casellas J, et al. Dual-phase ${ }^{99 m} T c-$ $\mathrm{MIBI}$ scintigraphy to assess calcimimetic effect in patients on haemodialysis with secondary hyperparathyroidism. Nucl Med Commun 2009;30:890-894.

108. Frank E, Watson W, Fujimoto S, De Andrade Filho P, Inman J, Simental A. Surgery versus imaging in non-localizing primary hyperparathyroidism: a cost-ffectiveness model. Laryngoscope 2020:130:E963E969.

109. Pata G, Casella C, Magri GC, Lucchini S, Panarotto MB, Crea N, et al. Financial and clinical implications

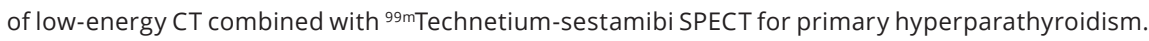
Ann Surg Oncol 2011;18:2555-2563. 


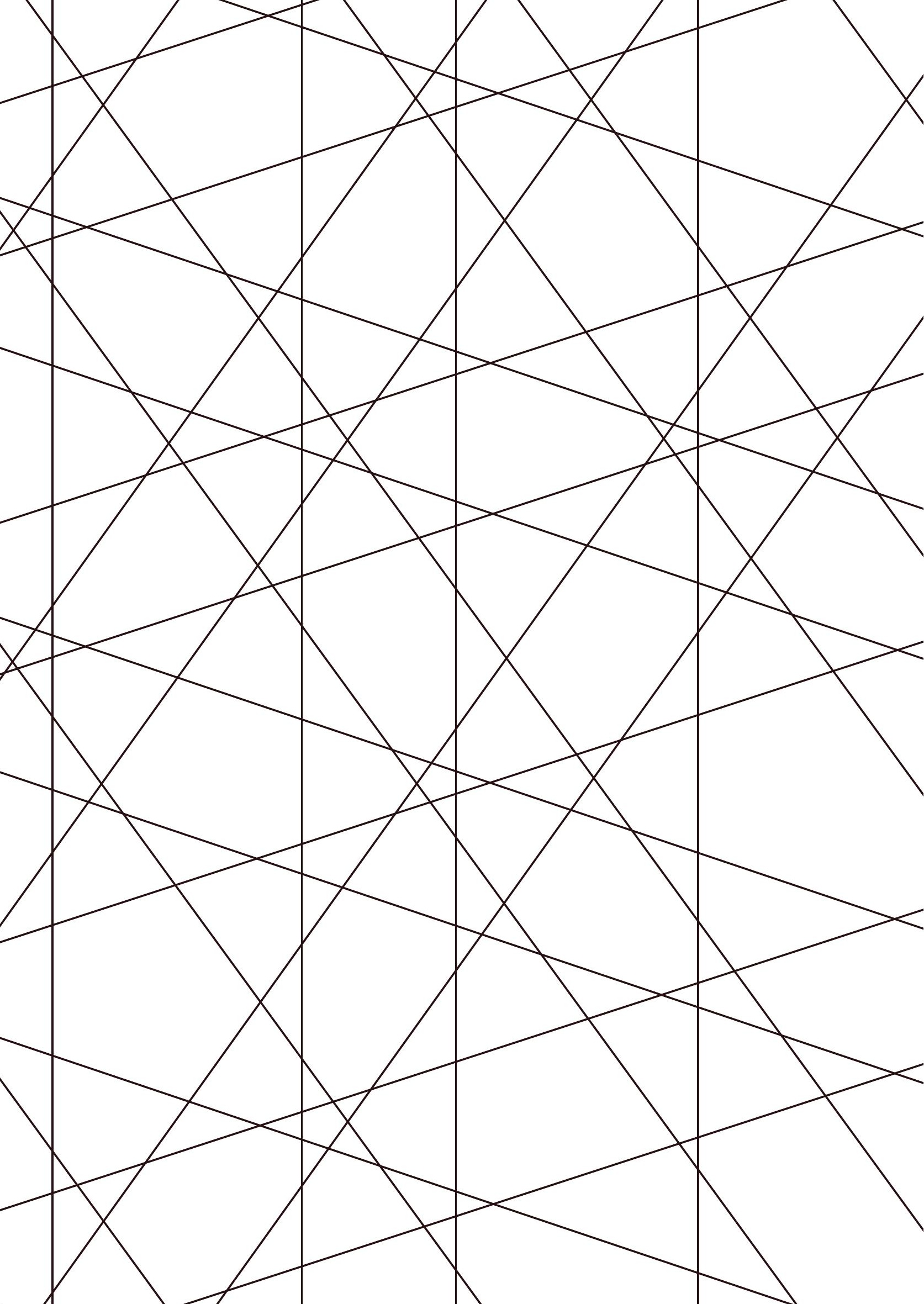




\section{CHAPTER 9}

Impact paragraph 
Primary hyperparathyroidism is an endocrine disorder in which one or more of the parathyroid glands in the neck produces too much parathyroid hormone. The disease is preferably treated by parathyroidectomy, i.e. surgical removal of the hyperfunctioning parathyroid gland(s). Accurate preoperative localization of abnormal glands is important to guide the surgeon, since finding those during surgery can be quite a challenge. In this thesis, parathyroid imaging with ${ }^{18} \mathrm{~F}$-fluorocholine $\left({ }^{18} \mathrm{~F}-\mathrm{FCH}\right)$ PET/CT was studied, a novel imaging technique to visualize hyperfunctioning parathyroid glands. The objectives of this thesis were to review the current scientific literature on the performance of ${ }^{18} \mathrm{~F}-\mathrm{FCH}$ PET/ $\mathrm{CT}$ to detect hyperfunctioning parathyroid glands, to optimize scan acquisition protocols and to explore the clinical implications of parathyroid localization using ${ }^{18} \mathrm{~F}-\mathrm{FCH}$ PET/CT.

Research to optimize imaging techniques for parathyroid localization in hyperparathyroidism is of clinical relevance in several ways. In the first place, primary hyperparathyroidism is a relatively common disease and its incidence has increased significantly in the past decades due to routine blood calcium level measurements. The prevalence in the general population has been estimated at 3 in 1000 and up to 21 in 1000 in postmenopausal females.[1,2] Furthermore, although nowadays most patients present without overt symptoms, the disease can lead to debilitating multi-organ involvement and decreased quality of life. Symptoms in hyperparathyroidism usually involve the skeleton (loss of bone density associated with fractures, skeletal deformities, bone pain), the kidneys (kidney stones, reduced renal function), the gastrointestinal tract (peptic ulcers, inflammation of the pancreas) and the cardiovascular system (high blood pressure, accelerated heart and blood vessel disease). Moreover, patients also experience symptoms which are more subjective, such as neurocognitive symptoms (sleep, concentration and memory problems, anxiety, depression), nonspecific abdominal pain, nausea and muscle weakness, which additionally contribute to the burden of disease. $[3,4]$

Parathyroidectomy remains the only curative treatment for primary hyperparathyroidism. Surgical excision of hyperfunctioning parathyroid glands has been shown to increase quality of life, determined with health related quality of life measuring instruments such as the SF-36 short form health survey as well as disease specific questionnaires.[5,6] Surgery is the first choice when certain diagnostic criteria are met (such as age $<50$ years, significant elevation of blood calcium levels, osteoporosis, vertebral fracture or kidney stones). However, studies have also demonstrated the positive effect of parathyroidectomy on quality of life in patients with milder and nonspecific symptoms.[4,7] Moreover, parathyroidectomy has been shown to be cost-effective compared to observation or treatment with medication for both symptomatic as well as asymptomatic disease.[8,9] The preferred surgical 
technique is minimally invasive parathyroidectomy instead of conventional bilateral neck exploration, which is a more extensive surgical procedure. Both surgical techniques have high success rates, but minimally invasive parathyroidectomy shortens operative and hospital time, reduces postoperative discomfort and is cosmetically superior due to reduction of incision length.[10] Preoperative imaging to localize all hyperfunctioning parathyroid glands is essential when performing minimally invasive surgery, since this procedure is only targeted at the preoperatively defined abnormal gland(s). Additionally, adequate preoperative visualization has been demonstrated to reduce overall costs, in particular because the cost-savings due to reduction of surgery duration and in-hospital length of stay, associated with minimally invasive surgery, outweighs the extra costs of advanced imaging without significantly decreasing the success rate of surgery.[11-13]

Apart from the abovementioned relevance for patients, the results of this thesis are more specifically of relevance for clinicians working in the field of hyperparathyroidism and nuclear medicine imaging. Although in recent years ${ }^{18} \mathrm{~F}-\mathrm{FCH}$ PET/CT has been increasingly used to localize hyperfunctioning parathyroid glands, there is no consensus on what scan acquisition protocols should be used. In the studies presented in chapter 3 and 4 of this thesis, different ${ }^{18} \mathrm{~F}-\mathrm{FCH}$ PET/CT scanning protocols were evaluated to optimize acquisition parameters, which is important to improve scan accuracy. We concluded from these studies that early scan acquisition is of additional value besides the standard acquisition at a later time point, because some hyperfunctioning parathyroid glands show fast tracer wash-out and therefore can be missed on late images. Moreover, with the help of dynamic ${ }^{18} \mathrm{~F}$-FCH PET imaging, we determined the optimal scanning time points for discrimination of hyperfunctioning parathyroid glands from other anatomic structures that can show uptake of ${ }^{18} \mathrm{~F}-\mathrm{FCH}$ and complicate reading of the scan. These results are especially relevant for nuclear medicine physicians to determine the optimal acquisition protocols on their department and for the development of standard procedure guidelines on PET/CT parathyroid imaging. At our department a standard dual-time-point ${ }^{18} \mathrm{~F}-\mathrm{FCH}$ PET/CT scan acquisition is already implemented.

Precise preoperative localization of the hyperfunctioning glands is essential for optimal surgical treatment. In chapter 5, the performance of ${ }^{18} \mathrm{~F}-\mathrm{FCH}$ PET/CT for detection and localization of hyperfunctioning parathyroid glands was studied as a first-line imaging modality in patients with primary hyperparathyroidism. ${ }^{18} \mathrm{~F}-\mathrm{FCH}$ PET/CT in this setting yielded excellent detection rates of $>90 \%$. These data are supported by the findings of the systematic review on ${ }^{18} \mathrm{~F}$-FCH PET/CT performance, presented in chapter 2 of this thesis. Although we concluded in this review that the level of evidence was moderate, mainly due to a low number of studies and heterogeneity between studies, these results were 
confirmed in recent systematic reviews with larger patient populations.[14,15] ${ }^{18} \mathrm{~F}-\mathrm{FCH}$ PET/ CT parathyroid imaging is often only performed when conventional imaging techniques are negative or equivocal. However, ${ }^{18} \mathrm{~F}-\mathrm{FCH}$ PET/CT is increasingly used as a first-line imaging modality, as it was applied in the aforementioned cohort study and implemented in our clinic. The findings of these studies are relevant both for nuclear medicine physicians as well as other medical professionals such as surgeons and endocrinologists who may order the PET/CT scans. It supports physicians to establish the optimal imaging routine in their clinic and to determine in which patient groups these imaging modalities are ideally implemented. Moreover, the results are relevant for further implementation of the technique as a first-line imaging modality in other nuclear medicine departments and adding the option of parathyroid imaging with ${ }^{18} \mathrm{~F}-\mathrm{FCH}$ PET/CT to clinical guidelines.

Regularly, ${ }^{18} \mathrm{~F}-\mathrm{FCH}$ PET/CT scans for parathyroid imaging demonstrate lesions which are not related to the parathyroid disorder, but the frequency and relevance of these findings are unknown. In chapter 6 of this thesis, we evaluated all incidental findings on ${ }^{18} \mathrm{~F}-\mathrm{FCH}$ PET/CT scans performed at our department and concluded that these abnormalities were commonly detected, present in more than half of the scans. Moreover, incidental malignant lesions were found in $2.6 \%$ of the patients, most frequently breast cancer. This overview of frequency and relevance of incidental findings on ${ }^{18} \mathrm{~F}-\mathrm{FCH}$ PET/CT especially facilitates the nuclear medicine physician with the interpretation of the scan and also guides clinicians in follow-up strategies.

All in all, from the results of this thesis and other scientific literature, it is clear that ${ }^{18} \mathrm{~F}$ $\mathrm{FCH} \mathrm{PET/CT} \mathrm{is} \mathrm{superior} \mathrm{in} \mathrm{detecting} \mathrm{hyperfunctioning} \mathrm{parathyroid} \mathrm{glands} \mathrm{compared} \mathrm{to}$ conventional imaging techniques. For some patients with hyperparathyroidism, the use of ${ }^{18} \mathrm{~F}-\mathrm{FCH}$ PET/CT may lead to faster cure, less symptoms, therefore higher quality of life, and could additionally save costs of medical treatment and follow-up imaging. Moreover, the findings in this thesis are not only clinically relevant, but also scientifically in supporting further research. For example to study in which patient groups, with parathyroid disorders other than primary hyperparathyroidism, imaging with ${ }^{18} \mathrm{~F}-\mathrm{FCH}$ $\mathrm{PET} / \mathrm{CT}$ is additionally useful. Also, since detection of hyperfunctioning parathyroid glands can still be a challenge in some patients, subsequent studies could determine if combining ${ }^{18} \mathrm{~F}-\mathrm{FCH}$ PET/CT with other upcoming imaging techniques such as MRI or multiphase contrast-enhanced CT is beneficial. Furthermore, the findings in this thesis could be useful for additional research on optimizing scanning protocols. It is to be expected that future research will further clarify the role of ${ }^{18} \mathrm{~F}-\mathrm{FCH}$ PET/CT in parathyroid imaging and will enshrine ${ }^{18} \mathrm{~F}-\mathrm{FCH}$ PET/CT as an important part of the clinical work-up of hyperparathyroidism. 


\section{References}

1. Adami S, Marcocci C, Gatti D. Epidemiology of primary hyperparathyroidism in Europe. J Bone Miner Res 2002;17 Suppl 2:N18-23.

2. Lundgren E, Rastad J, Thrufjell E, Akerstrom G, Ljunghall S. Population-based screening for primary hyperparathyroidism with serum calcium and parathyroid hormone values in menopausal women. Surgery 1997;121:287-294.

3. Bilezikian JP, Bandeira L, Khan A, Cusano NE. Hyperparathyroidism. Lancet 2018;391:168-178.

4. Zhu CY, Nguyen DT, Yeh MW. Who benefits from treatment of primary hyperparathyroidism? Surg Clin North Am 2019;99:667-679.

5. Coker LH, Rorie K, Cantley L, Kirkland K, Stump D, Burbank N, et al. Primary hyperparathyroidism, cognition, and health-related quality of life. Ann Surg 2005;242:642-650.

6. Gopinath P, Sadler GP, Mihai R. Persistent symptomatic improvement in the majority of patients undergoing parathyroidectomy for primary hyperparathyroidism. Langenbecks Arch Surg 2010;395:941946.

7. McDow AD, Sippel RS. Should symptoms be considered an indication for parathyroidectomy in primary hyperparathyroidism? Clin Med Insights Endocrinol Diabetes 2018;11:1179551418785135.

8. Zanocco K, Heller M, Sturgeon C. Cost-effectiveness of parathyroidectomy for primary hyperparathyroidism. Endocr Pract 2011;17 Suppl 1:69-74.

9. Zanocco KA, Wu JX, Yeh MW. Parathyroidectomy for asymptomatic primary hyperparathyroidism: a revised cost-effectiveness analysis incorporating fracture risk reduction. Surgery 2017;161:16-24.

10. Ishii H, Mihai R, Watkinson JC, Kim DS. Systematic review of cure and recurrence rates following minimally invasive parathyroidectomy. BJS Open 2018;2:364-370.

11. Fahy BN, Bold RJ, Beckett L, Schneider PD. Modern parathyroid surgery: a cost-benefit analysis of localizing strategies. Arch Surg 2002;137:917-22; discussion 922-3.

12. Ruda J, Stack BC,Jr, Hollenbeak CS. The cost-effectiveness of sestamibi scanning compared to bilateral neck exploration for the treatment of primary hyperparathyroidism. Otolaryngol Clin North Am 2004;37:855-70, x-xi.

13. Frank E, Watson W, Fujimoto S, De Andrade Filho P, Inman J, Simental A. Surgery versus imaging in non-localizing primary hyperparathyroidism: a cost-effectiveness model. Laryngoscope 2020:130:E963E969.

14. Treglia G, Piccardo A, Imperiale A, Strobel K, Kaufmann PA, Prior JO, et al. Diagnostic performance of choline PET for detection of hyperfunctioning parathyroid glands in hyperparathyroidism: a systematic review and meta-analysis. EurJ Nucl Med Mol Imaging 2018;46:751-765.

15. Evangelista L, Ravelli I, Magnani F, lacobone M, Giraudo C, Camozzi V, et al. 18F-choline PET/CT and PET/MRI in primary and recurrent hyperparathyroidism: a systematic review of the literature. Ann Nucl Med 2020;34:601-619. 


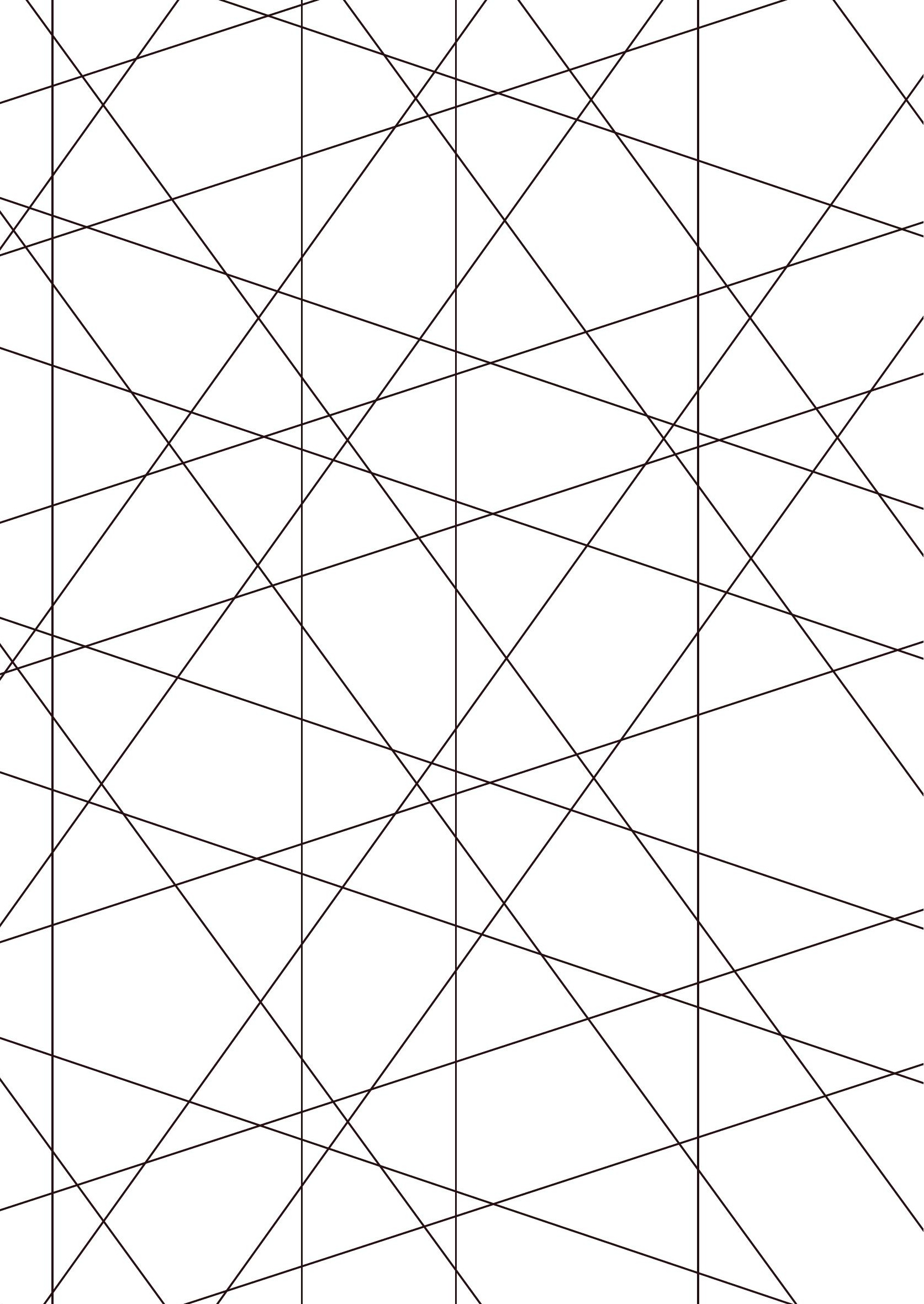


APPENDICES 


\section{Appendix 1 - Nederlandse samenvatting}

In dit proefschrift werd de beeldvormende techniek ${ }^{18} \mathrm{~F}$-fluorocholine PET/CT (in het vervolg afgekort tot ${ }^{18} \mathrm{~F}$-FCH PET/CT) onderzocht voor het afbeelden van te snel werkende bijschildklieren bij patiënten lijdend aan hyperparathyreoïdie. Alvorens dieper in te gaan op de in deze studies gevonden resultaten, wordt hieronder eerst meer uitleg gegeven over de bijschildklieren, de aandoening hyperparathyreoïdie en de scantechniek ${ }^{18} \mathrm{~F}$ FCH PET/CT.

\section{Achtergrondinformatie}

Bijschildklieren zijn orgaantjes die zich bevinden in de hals, gelegen achter de schildklier, onder het niveau van het strottenhoofd. Het zijn kleine, bolronde structuren van vaak nog geen halve centimeter groot, normaliter vier in aantal. De bijschildklieren hebben een belangrijke functie bij het in evenwicht houden van calciumwaarden in het bloed. Wanneer het calciumniveau te laag is, wordt dit bemerkt door de bijschildklieren die vervolgens bijschildklierhormoon (PTH) uitscheiden. Dit hormoon zorgt ervoor dat de calciumspiegel in het bloed stijgt door calcium vrij te maken uit het bot, door extra calcium op te nemen uit voedsel via de darmen en door ervoor te zorgen dat er minder calcium wordt uitgescheiden door de nieren.

Wanneer de bijschildklieren te veel PTH aanmaken spreekt men van hyperparathyreoïdie. Als de oorzaak van de hoge PTH-uitscheiding bij de bijschildklieren zelf ligt, wordt dit een primaire hyperparathyreoïdie genoemd; een aandoening die bij ongeveer in 3 op de 1000 mensen voorkomt. De hoge PTH-secretie bij primaire hyperparathyreoïdie wordt meestal veroorzaakt door een goedaardig gezwel (adenoom) in een enkele bijschildklier (ca. $89 \%$ van de gevallen), minder vaak door meerdere adenomen of een algehele te snelle groei (hyperplasie) van de bijschildklieren (ca. 10\%) en in zeer zeldzame gevallen $(<1 \%)$ door een kwaadaardige bijschildkliertumor. In veel gevallen hebben mensen met primaire hyperparathyreoïdie weinig klachten, hoewel de ziekte kan leiden tot een verscheidenheid aan lichamelijke symptomen zoals botbreuken door botontkalking, spierzwakte, buikpijn, misselijkheid, nierstenen en hart- en vaatziekten, maar ook tot psychische klachten zoals slaap-, concentratie- en geheugenproblemen, angstgevoelens en depressie.

Hoewel primaire hyperparathyreoïdie medicamenteus behandeld kan worden, is operatieve verwijdering van de te snel werkende bijschildklieren de enige manier om de ziekte definitief te genezen. In het verleden was een halsexploratie de standaard operatie 
hiervoor, waarbij de hals na incisie aan beide zijden grondig geïnspecteerd wordt op vergrote bijschildklieren. Tegenwoordig wordt echter meestal een minimaal invasieve techniek gebruikt, waarbij gericht op de aangedane bijschildklier wordt afgegaan middels een kleine incisie. Bij deze aanpak is het uiteraard wel van belang dat van tevoren duidelijk is welke bijschildklier verwijderd moet worden en waar deze zich exact bevindt. Hiervoor zijn verschillende beeldvormende technieken beschikbaar. Daarnaast bevindt een bijschildklier zich soms niet op de typische plek achter de schildklier en wordt dan ectopisch genoemd. Wanneer een ectopische bijschildklier zich tot adenoom ontwikkelt en operatief verwijderd moet worden, zal deze met een standaard halsexploratie niet altijd gevonden kunnen worden en zijn mogelijk andere operatietechnieken nodig. Derhalve is, in het bijzonder in deze gevallen, een accurate preoperatieve lokalisatie essentieel.

Er zijn diverse beeldvormende technieken beschikbaar om de aangedane bijschildklieren te lokaliseren. Radiologische technieken zijn vooral gericht op detectie van een afwijkende anatomie, zoals bijschildklieren die vergroot zijn. Nucleair geneeskundige beeldvorming is daarentegen gericht op het in beeld brengen van functionaliteit. Hierbij wordt een radioactieve stof (tracer) toegediend, welke zich in de patiënt concentreert ter plaatse van het te onderzoeken ziekteproces en welke vervolgens met speciale camera's gelokaliseerd kan worden. Voor bijschildklierdetectie wordt de laatste jaren steeds vaker de gevoelige nucleair geneeskundige scantechniek positron-emissie tomografie (PET) gebruikt. Dit wordt gecombineerd met de radiologische techniek computertomografie (CT) voor een driedimensionale anatomische afbeelding van het lichaam om zo de verhoogde activiteit te kunnen lokaliseren. De voor bijschildklierlokalisatie gebruikte tracer ${ }^{18} \mathrm{~F}-\mathrm{FCH}$ is gebaseerd op choline, een molecuul dat opgenomen wordt door weefsel met een hoge celdelingsactiviteit, zoals bijschildklieradenomen.

Omdat ${ }^{18} \mathrm{~F}-\mathrm{FCH}$ PET/CT een nog relatief weinig gebruikte scantechniek is voor bijschildklierdetectie werden in dit proefschrift verschillende facetten van deze techniek onderzocht om de scanmethode te optimaliseren en te beoordelen wat in de klinische praktijk de waarde is van bijschildklierdetectie middels ${ }^{18} \mathrm{~F}-\mathrm{FCH}$ PET/CT.

\section{Samenvatting studies}

In DEEL 1 van het proefschrift werd het onderwerp geïntroduceerd. Na een beknopte inleiding in hoofdstuk 1, werd in hoofdstuk 2 een systematische review beschreven waarin de bestaande medische literatuur met betrekking tot de opbrengst van bijschildklierdetectie met ${ }^{18} \mathrm{~F}-\mathrm{FCH}$ PET/CT tegen het licht gehouden werd. Hierbij waren 
we geïnteresseerd in de detectieratio die in deze studies gevonden werd, dit wil zeggen: het percentage van alle bij operatie verwijderde bijschildklieradenomen dat correct gelokaliseerd werd op de ${ }^{18} \mathrm{~F}-\mathrm{FCH}$ PET/CT scan. Dit werd bekeken per patiënt en ook per bijschildklieradenoom, daar er soms meerdere bijschildklieren aangedaan zijn. Alle relevante artikelen uit de wetenschappelijke literatuur werden bekeken en gescoord op wetenschappelijke waarde. De resultaten van de geselecteerde studies werden uit de artikelen overgenomen en vervolgens werd de gemiddelde detectieratio berekend, gewogen naar het aantal patiënten dat meedeed per studie. Er werden in totaal 11 relevante studies gevonden, waarvan er 9 geschikt bleken voor analyse van de resultaten. Er werd een hoge gemiddelde detectieratio gevonden van 97\% (per patiënt) en 94\% (per bijschildklier). Dit wil zeggen dat er met deze scantechniek slechts weinig snelwerkende bijschildklieren worden gemist. Wel werd geconstateerd dat het wetenschappelijke niveau van de artikelen vaak matig was en de onderzochte patiëntgroepen tussen de studies veelal niet goed vergelijkbaar waren. Wij concludeerden dan ook dat er meer onderzoek gewenst was.

In DEEL 2 van het proefschrift werd een tweetal door ons uitgevoerde studies beschreven waarin verschillende manieren van uitvoering van een ${ }^{18} \mathrm{~F}-\mathrm{FCH}$ PET/CT scan zijn onderzocht. Er kunnen namelijk verschillende scanprotocollen gebruikt worden en in de wetenschappelijke literatuur is er nog geen volledige overeenstemming welke hiervan het meest geschikt is. Zoals bovenbeschreven vindt er in bijschildklieradenomen stapeling plaats van de tracer ${ }^{18} \mathrm{~F}-\mathrm{FCH}$. Vervolgens blijft echter niet alles in de cellen van het bijschildklieradenoom, maar wordt ook een deel van de tracer weer losgelaten in het bloed; dit wordt wash-out genoemd. Het is bekend dat sommige bijschildklieradenomen een snelle wash-out hebben waardoor deze gemist kunnen worden op standaard opnamen (gewoonlijk verricht op 60 minuten na injectie van de tracer). In hoofdstuk $\mathbf{3}$ hebben we onderzocht wat de toegevoegde waarde is van extra opnamen vroeg na injectie van de tracer, namelijk na 5 minuten. In deze studie werden in totaal 64 patiënten geïncludeerd, allen met een bewezen bijschildklieradenoom/-hyperplasie en gescand op zowel het vroege als late tijdstip. Bij vergelijking van de 2 opnamen werd $20 \%$ van de snelwerkende bijschildklieren beter gevisualiseerd op de vroege opnamen, $6 \%$ op de late en in $2 \%$ was de bijschildklier alleen zichtbaar op de late opnamen. In de overige $62 \%$ was er een vergelijkbare visualisatie op vroege en late beelden. Wij concludeerden uit deze studie dat bijschildklieren inderdaad verschillende wash-out patronen vertonen en dat het verrichten van een scan op twee tijdstippen van toegevoegde waarde is om snelwerkende bijschildklieren optimaal te detecteren. 
Naast opname van ${ }^{18} \mathrm{~F}-\mathrm{FCH}$ in snelwerkende bijschildklieren wordt er ook stapeling van de tracer gezien in andere anatomische structuren zoals speekselklieren, schildklier, lever, milt, beenmerg en regelmatig ook lymfeklieren. Stapeling in de schildklier kan de interpretatie van een scan bemoeilijken doordat de bijschildklieren hier meestal vlak achter liggen en een actieve lymfeklier kan de foute indruk geven van een bijschildklieradenoom. In hoofdstuk $\mathbf{4}$ werd een studie beschreven waarin we met behulp van een dynamische scanmethode bekeken hebben op welk scantijdstip de snelwerkende bijschildklieren het beste onderscheiden konden worden van schildklier dan wel actieve lymfeklieren. In dit onderzoek werden 101 patiënten, allen met een bewezen bijschildklieradenoom/-hyperplasie, gedurende 20 minuten gescand (dynamische scan). Actieve lymfeklieren konden het best van snelwerkende bijschildklieren onderscheiden worden in de eerste 5 minuten na injectie van de tracer, alhoewel geen eenduidige afkapwaarde kon worden vastgesteld. Tracer wash-out van de schildklier was meestal sneller dan die van de bijschildklier en het beste tijdstip om de activiteit in de snelwerkende bijschildklier te onderscheiden van schildklieractiviteit was minimaal 10 minuten na injectie van de tracer. Een enkel scantijdstip voor een optimaal onderscheid tussen de structuren kon dus niet bepaald worden, maar wij concludeerden wel dat dynamische opnamen kunnen bijdragen aan het karakteriseren van snelwerkende bijschildklieren.

Het intermezzo van dit proefschrift beschrijft een interessante casus van een patiënt met chronisch nierfalen en hyperparathyreoïdie. De ${ }^{18} \mathrm{~F}-\mathrm{FCH}$ PET/CT die bij deze patiënt werd verricht toonde, naast een mooi voorbeeld van een viertal snelwerkende bijschildklieren op de typische locaties passend bij hyperplasie, ook een verhoogde activiteit in het skelet. Dit werd geduid als een renale osteodystrofie, een ziektebeeld dat gerelateerd is aan chronisch nierfalen en leidt tot botabnormaliteiten zoals botverharding of botontkalking met kans op botbreuken, botpijnen en soms zelfs afsterving van bot. Renale osteodystrofie afgebeeld met ${ }^{18} \mathrm{~F}-\mathrm{FCH}$ PET/CT werd niet eerder beschreven in de wetenschappelijke literatuur en is een ziektebeeld dat relevant is om te onderkennen voorafgaand aan bijschildklierchirurgie.

In DEEL 3 van het proefschrift werden verschillende klinische aspecten van ${ }^{18} \mathrm{~F}-\mathrm{FCH}$ PET/ CT bij hyperparathyreoïdie belicht. In hoofdstuk $\mathbf{5}$ werd een groot cohort van 271 patiënten met primaire hyperparathyreoïdie bestudeerd, allen gescand in ons eigen instituut met gebruik van ${ }^{18} \mathrm{~F}-\mathrm{FCH}$ PET/CT als eerstelijns beeldvormingsmodaliteit, wat wil zeggen dat de patiënten geen eerdere conventionele beeldvorming ondergingen. In totaal werden 139 van de patiënten geopereerd en de ${ }^{18} \mathrm{~F}-\mathrm{FCH}$ PET/CT detectieratio die berekend werd in deze groep was 90\% (per patiënt) en 96\% (per bijschildklier), 
vergelijkbaar met de getallen die genoemd worden in de wetenschappelijke literatuur. Naar aanleiding van deze resultaten concludeerden wij dat ${ }^{18} \mathrm{~F}-\mathrm{FCH}$ PET/CT geschikt is voor gebruik als eerstelijns beeldvormingsmodaliteit in het kader van preoperatieve planning bij patiënten met primaire hyperparathyreoïdie.

Regelmatig worden op ${ }^{18} \mathrm{~F}-\mathrm{FCH}$ PET/CT scans nevenbevindingen waargenomen; afwijkingen die niet direct gerelateerd zijn aan de indicatie waarvoor de scan verricht werd. Dit kunnen allerlei afwijkingen betreffen, zowel op PET als op CT, zoals ontstekingsprocessen en goedaardige gezwellen, maar ook kwaadaardige tumoren kunnen zo per toeval worden ontdekt. Om de frequentie en relevantie van nevenbevindingen op ${ }^{18} \mathrm{~F}$-FCH PET/CT voor bijschildklierdetectie in kaart te brengen werd in hoofdstuk 6 een totaal van $388{ }^{18} \mathrm{~F}-\mathrm{FCH}$ PET/CT scans bekeken. Nevenbevindingen waren zichtbaar op 226 scans (58\%), met abnormaal verhoogde stapeling van ${ }^{18} \mathrm{~F}$-FCH op 85 scans (22\%) en in 10 patiënten (2,6\%) werd een kwaadaardige tumor gedetecteerd. Dit betrof met name borstkanker (7 patiënten) en daarnaast long-, schildklier- en huidkanker. De resultaten van dit onderzoek dragen bij aan kennis over de distributie van ${ }^{18} \mathrm{~F}-\mathrm{FCH}$ in het lichaam en helpt artsen bij het beoordelen van ${ }^{18} \mathrm{~F}-\mathrm{FCH}$ PET/CT en bij het bepalen welke afwijkingen verder onderzoek behoeven of juist kunnen worden genegeerd.

In DEEL 4 van het proefschrift werden de resultaten samengevat (hoofdstuk 7) met vervolgens een uitgebreide discussie over de verschillende aspecten van ${ }^{18} \mathrm{~F}-\mathrm{FCH}$ PET/ CT (hoofdstuk 8) en een toelichting over de maatschappelijke en wetenschappelijke impact van het onderzoek (hoofdstuk 9).

\section{Conclusie}

Al met al laten de resultaten van de studies in dit proefschrift zien dat ${ }^{18} \mathrm{~F}-\mathrm{FCH}$ PET/ CT een zeer geschikte scantechniek is voor het detecteren en lokaliseren van snelwerkende bijschildklieren bij patiënten met hyperparathyreoïdie. De resultaten zijn in overeenstemming met de beschikbare wetenschappelijke literatuur die bovendien aantonen dat ${ }^{18} \mathrm{~F}-\mathrm{FCH}$ PET/CT superieur is aan conventionele beeldvormingstechnieken, zoals echografie en ${ }^{99 \mathrm{~m} T c-s e s t a m i b i}$ scintigrafie, maar ook minstens zo accuraat is als andere nieuwe technieken, zoals MRI en 4D-CT (CT met meerdere contrastfasen). ${ }^{18} \mathrm{~F}$ FCH PET/CT is geschikt gebleken als tweedelijnstechniek, in gevallen dat conventionele beeldvorming geen snelwerkende bijschildklieren hebben kunnen aantonen, maar kan eveneens adequaat worden ingezet als eerstelijnstechniek, hoewel kosteneffectiviteitsanalyses tot dusver ontbreken. Hiernaast hebben de studies in dit proefschrift bijgedragen aan het optimaliseren van scantechnieken en hebben deze een 
beter inzicht gegeven in de frequentie en relevantie van toevalsbevindingen op ${ }^{18} \mathrm{~F}-\mathrm{FCH}$ PET/CT. Hoewel verschillende aspecten rondom ${ }^{18} \mathrm{~F}-\mathrm{FCH}$ PET/CT nog verder opgehelderd kunnen worden, zoals een goede één-op-één vergelijking met andere scantechnieken (inclusief het kostenaspect) en verdere optimalisatie van scanprotocollen, is het de verwachting dat ${ }^{18} \mathrm{~F}-\mathrm{FCH} \mathrm{PET} / \mathrm{CT}$ in de toekomst een prominente plaats zal innemen bij het preoperatief lokaliseren van snelwerkende bijschildklieren bij patiënten met hyperparathyreoïdie. 


\section{Appendix 2 - Abbreviations}

\begin{aligned} & \hline Radio-isotopes \\ & \hline${ }^{11} \mathrm{C}$ Carbon-11 \\ &${ }^{123}$ lodine-123 \\ &${ }^{18} \mathrm{~F}$ Fluorine-18 \\ &${ }^{99 \mathrm{~m} T \mathrm{C}}$ Technetium-99m (metastable) \\ & \hline\end{aligned}

\begin{tabular}{ll}
\hline \multicolumn{2}{l}{ Radiopharmaceuticals } \\
\hline${ }^{11} \mathrm{C}-\mathrm{CH}$ & ${ }^{11} \mathrm{C}$-choline \\
${ }^{18} \mathrm{~F}-\mathrm{DOPA}$ & ${ }^{18} \mathrm{~F}$-dihydroxyphenylalanine \\
${ }^{18} \mathrm{~F}-\mathrm{FCH}$ & ${ }^{18} \mathrm{~F}$-fluorocholine / fluoro(methyl)choline \\
${ }^{18} \mathrm{~F}-\mathrm{FDG}$ & ${ }^{18} \mathrm{~F}$-fluordeoxyglucose \\
${ }^{18} \mathrm{~F}-\mathrm{FEC}$ & ${ }^{18} \mathrm{~F}$-fluoroethylcholine \\
${ }^{18} \mathrm{~F}$-FET & ${ }^{18} \mathrm{~F}$-fluoroethyl-L-thyrosine \\
\hline
\end{tabular}

\begin{tabular}{ll}
\hline Other abbreviations \\
\hline AC & attenuation-corrected \\
CI & confidence interval \\
CPS & counts per second \\
CT & computed tomography \\
MEN & multiple endocrine neoplasia syndrome \\
MIP & maximum-intensity projection \\
MRI & magnetic resonance imaging \\
p.i. & post injection \\
PET & positron emission tomography \\
PTH & parathyroid hormone \\
ROC & receiver operating characteristic \\
SD & standard deviation \\
SPECT & single-photon emission computed tomography \\
SUV & standardized uptake value \\
US & ultrasonography \\
VOI & volume of interest \\
\hline
\end{tabular}




\begin{tabular}{ll}
\hline Units & \\
\hline $\mathrm{A}$ & ampere \\
$\mathrm{Bq}$ & bequerel \\
$\mathrm{Ci}$ & curie \\
$\mathrm{g}$ & gram \\
$\mathrm{h}$ & hour \\
$\mathrm{L}$ & liter \\
$\mathrm{m}$ & meter \\
min & minute \\
mol & molar \\
$\mathrm{S}$ & second \\
SV & sievert \\
$\mathrm{T}$ & tesla \\
$\mathrm{V}$ & volt \\
\hline
\end{tabular}

\section{Conversion factors}

\begin{tabular}{ll}
\hline Serum calcium & $1 \mathrm{mmol} / \mathrm{L} \rightarrow 4.0 \mathrm{mg} / \mathrm{dL}$ \\
PTH & $1 \mathrm{pmol} / \mathrm{L} \rightarrow 9.43 \mathrm{pg} / \mathrm{mL}$ \\
Radioactivity & $1 \mathrm{MBq} \rightarrow 0.027 \mathrm{mCi}$
\end{tabular}




\section{Appendix 3 - Dankwoord}

Bedankt beoordelingscommissie, prof. dr. Mottaghy, prof. dr. Bouvy, prof. dr. Valk en prof. dr. Glaudemans, dat jullie de moeite hebben willen nemen dit proefschrift te beoordelen.

Bedankt prof. dr. Schaper, Nicolaas, dat je me niet vergeten was toen ik zo'n 3 jaar na mijn vertrek uit Maastricht bij je aanklopte en vroeg of je mijn promotor wilde zijn. Helaas zijn onze subsidieaanvragen voor het onderzoek naar TNFa-scintigrafie bij Charcot-artropathie destijds nooit gehonoreerd, maar gelukkig verliep het huidige promotietraject een stuk soepeler. Bedankt voor je uitstekende begeleiding, het delen van je ruime ervaring en je steeds eindeloze enthousiasme.

Bedankt Maurits, zonder jouw voorwerk met het implementeren van choline PET op onze afdeling en opzet van scanprotocollen was de totstandkoming van dit proefschrift een stuk lastiger geweest. Bedankt voor je begeleiding als copromotor, je altijd scherpe inhoudelijke commentaren en je gezelligheid op de werkvloer. Helaas per 2021 wat meer op afstand nu je naar het Antoni van Leeuwenhoek bent vertrokken. Ik wens je veel succes daar.

Bedankt Remco, eveneens voor je begeleiding als copromotor. Naast je inhoudelijke bijdrage aan de teksten ook bedankt voor het beter leesbaar maken ervan en natuurlijk voor je professionele hulp met de figuren.

Bedankt Friso, dat je mij sinds dag één dat ik kwam werken in Alkmaar motiveerde te promoveren en daar niet mee op zal houden totdat het boekje fysiek op je bureau ligt. Uiteraard ook bedankt voor je overige bijdragen aan alle stukken.

Bedankt Henna, wellicht wat minder direct betrokken geweest bij het schrijven van de artikelen maar des te meer als collega nucleair geneeskundige.

Daarnaast ook dank aan de rest van de afdeling; laboranten, secretariaat, fysici, cyclotrongroep en apothekers. Bedankt voor alle hulp en de prettige sfeer op de afdeling wat dit proefschrift ten goede is gekomen.

Bedankt Jochem, dat je mijn paranimf wilde zijn. Naast dat je hebt bijgedragen aan een onvergetelijke opleidingsperiode in Maastricht ben je ook daarna altijd een goede vriend gebleven. Hopelijk is er snel weer de mogelijkheid om samen een mooi congres te bezoeken! 
Bedankt Jelle, eveneens dat je mijn paranimf wilde zijn. Daarnaast kan ik me geen betere zwager bedenken en wil ik je ook bedanken voor de goede zorgen voor Josien.

Bedankt alle vrienden en vriendinnen, voor het regelmatige verzetten van zinnen, met een drankje in de kroeg, op het terras of gewoon thuis. Bedankt allen die zich aangesproken voelen als ik roep: 'Proost!'

Bedankt familie, ouders Henk en Paula, schoonouders Piet en Margaret, zusje Anneloes en zusje Josien, dat jullie me altijd hebben genomen voor wie ik ben en dat jullie mij in elk geval het idee geven dat ik altijd kan aankloppen.

En veel dank mijn lieve Tamara, jij bent het doel in mijn leven, jij bent mijn inspiratie. 


\section{Appendix 4 - List of publications}

1. Wondergem M, van der Zant FM, Broos WAM, Knol RJ. Matched-pair comparison of ${ }^{18} \mathrm{~F}$-DCFPyL PET/CT and ${ }^{18} \mathrm{~F}$-PSMA-1007 PET/CT in 240 prostate cancer patients; inter-reader agreement and lesion detection rate of suspected lesions. J Nucl Med 2021:epub ahead of print.

2. Govers S, van der Zant FM, Wondergem M, Broos WAM, Knol RJJ. "Skull on fire": monostotic Paget disease of the skull bone. Clin Nucl Med 2021;46:55-57.

3. Broos WAM, Wondergem M, van der Zant FM, Schaper NC, Knol RJJ. Dynamic ${ }^{18} \mathrm{~F}$-fluorocholine PET/CT for parathyroid imaging in patients with primary hyperparathyroidism. Nucl Med Commun 2020;41:776-782.

4. van der Zant FM, Maurits W, Lazarenko SV, Broos WAM, Umans VAWM, Cornel JH, et al. Relation between pharmacological stress ECG, ${ }^{13} \mathrm{NH}_{3}$-PET/CT outcome and the occurrence of cardiac events during follow-up in women with chest pain. Nucl Med Commun 2020;41:783-789.

5. Wondergem M, van der Zant FM, Broos WAM, Knol RJJ. Clinical impact of PSMA PET in biochemically recurrent prostate cancer; a review of the literature. Tijdschr Urol 2020;10:109-121.

6. Bernsen MLE, van der Zant FM, Wondergem M, Broos WAM, Knol RJJ. Incidental breast carcinoma detected with ${ }^{13} \mathrm{~N}-\mathrm{NH}_{3}$ cardiac PET imaging. Clin Nucl Med 2020;45:e252-e253.

7. Meijer D, Wondergem M, Knol RJJ, Broos WAM, van der Zant FM. ${ }^{18}$ F-DCFPyL uptake in an incidentally detected follicular lymphoma by PET/CT performed for biochemically recurrent prostate cancer. Clin Nucl Med 2020;45:e96-e97.

8. Wondergem M, van der Zant FM, Broos WAM, Roeleveld TA, Donker R, Ten Oever D, et al. ${ }^{18} \mathrm{~F}$-DCFPyL PET/CT for primary staging in 160 high-risk prostate cancer patients; metastasis detection rate, influence on clinical management and preliminary results of treatment efficacy. Eur J Nucl Med Mol Imaging 2021;48:521-531.

9. Verberne SJ, Kuiper JWP, Broos WAM, Temmerman OPP. Systematic review on reproducibility of nuclear imaging in the assessment of painful hip and knee replacements. SN Compr Clin Med 2020;2:1491-1504.

10. Broos WAM, Wondergem M, Knol RJJ, van der Zant FM. Parathyroid imaging with ${ }^{18} \mathrm{~F}$-fluorocholine PET/CT as a first-line imaging modality in primary hyperparathyroidism: a retrospective cohort study. EJNMMI Res 2019;9:72.

11. Broos WAM, Wondergem M, van der Zant FM, Knol RJJ. Dual-time-point ${ }^{18}$ F-fluorocholine PET/CT in parathyroid imaging. J Nucl Med 2019;60:1605-1610. 
12. Wondergem M, Srbljin S, van der Zant FM, Broos WAM, Knol RJJ. ${ }^{18} \mathrm{~F}$-DCFPyL PET/CT in a single large metastasis with prostate-specific antigen level of $856 \mathrm{ng} / \mathrm{mL}$. Clin Nucl Med 2019;44:e588-e589.

13. Wondergem M, van der Zant F, Broos W, Knol R. Increased PSMA expression in castration-resistant prostate cancer metastases 3 months after initiation of enzalutamide indicated by ${ }^{18}$ F-DCFPyL PET/CT. Clin Nucl Med 2019;44:582-584.

14. Broos WAM, van der Zant FM, Knol RJJ, Wondergem M. Choline PET/CT in parathyroid imaging: a systematic review. Nucl Med Commun 2019;40:96-105.

15. Broos WAM, Kocken M, van der Zant FM, Knol RJJ, Wondergem M. Metastasized ${ }^{18} \mathrm{~F}$-DCFPyL-negative prostatic adenocarcinoma without neuroendocrine differentiation. Clin Nucl Med 2018;43:120-122.

16. Broos WAM, van der Zant FM, Wondergem M, Knol RJJ. Accuracy of ${ }^{18} \mathrm{~F}-\mathrm{NaF}$ PET/CT in bone metastasis detection and its effect on patient management in patients with breast carcinoma. Nucl Med Commun 2018;39:325-333.

17. Broos WAM, Wondergem M, van der Zant FM, Knol RJJ. Tertiary hyperparathyroidism with renal osteodystrophy on ${ }^{18}$ F-fluorocholine PET/CT. Clin Nucl Med 2018;43:766768.

18. Kwee RM, Broos WA, Brans B, Walenkamp GH, Geurts J, Weijers RE. Added value of ${ }^{18}$ F-FDG PET/CT in diagnosing infected hip prosthesis. Acta Radiol 2018;59:569-576.

19. Broos WAM, Mottaghy FM, Brans B. Nuclear Medicine Imaging of Lower Leg Injuries. In: Glaudemans AWJM, editor. Nuclear Medicine and Radiologic Imaging in Sports Injuries. Berlin, Heidelberg: Springer-Verlag; 2015. p. 743-755. 


\section{Appendix 5 - Curriculum vitae}

De auteur van dit proefschrift, Walterus (Wouter) Adrianus Maria Broos, werd geboren op 21 september 1986 te Roosendaal en Nispen. Na het behalen van het gymnasiumexamen aan het Norbertuscollege te Roosendaal begon hij in 2005 met de studie Geneeskunde aan de Universiteit Maastricht. De coschappen werden doorlopen in het Catharina Ziekenhuis te Eindhoven en de semi-artsstage in het laatste studiejaar op de afdeling orthopedie van het toenmalige Atrium Medisch Centrum te

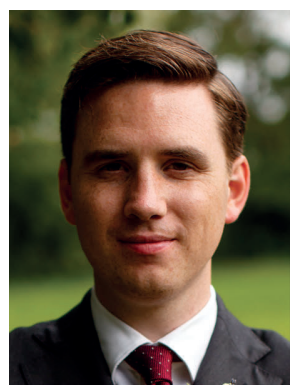
Heerlen. Het artsexamen werd behaald op 31 juli 2011.

Datzelfde jaar startte hij met de opleiding tot Nucleair Geneeskundige in het Maastricht Universitair Medisch Centrum met als opleider dr. B. Brans. Alhier werd de eerste wetenschappelijke interesse gewekt die vooral op het orthopedisch vlak gericht was (beeldvorming bij geïnfecteerde gewrichtsimplantaten), maar ook reeds raakvlakken met de endocrinologie had (beeldvorming bij Charcot-artropathie).

De laatste maanden van de opleiding tot Nucleair Geneeskundige werden gevolgd in het Medisch Centrum Alkmaar (thans Noordwest Ziekenhuisgroep) met als opleider dr. F.M. van der Zant. De opleiding werd afgerond op 1 oktober 2016 waarna Wouter toetrad tot de vakgroep Nucleaire Geneeskunde van Noordwest, met als voornaamste aandachtsgebieden de nucleaire beeldvorming binnen de oncologie, hematologie, orthopedie en endocrinologie. In 2017 startte hij met een onderzoekstraject naar de detectie van hyperfunctionerende bijschildklieren middels ${ }^{18} \mathrm{~F}$-fluorocholine PET/CT met als promotor prof. dr. N.C. Schaper, hoogleraar endocrinologie verbonden aan het Maastricht Universitair Medisch Centrum. Dit onderzoek heeft uiteindelijk geleid tot het proefschrift dat voor u ligt. 



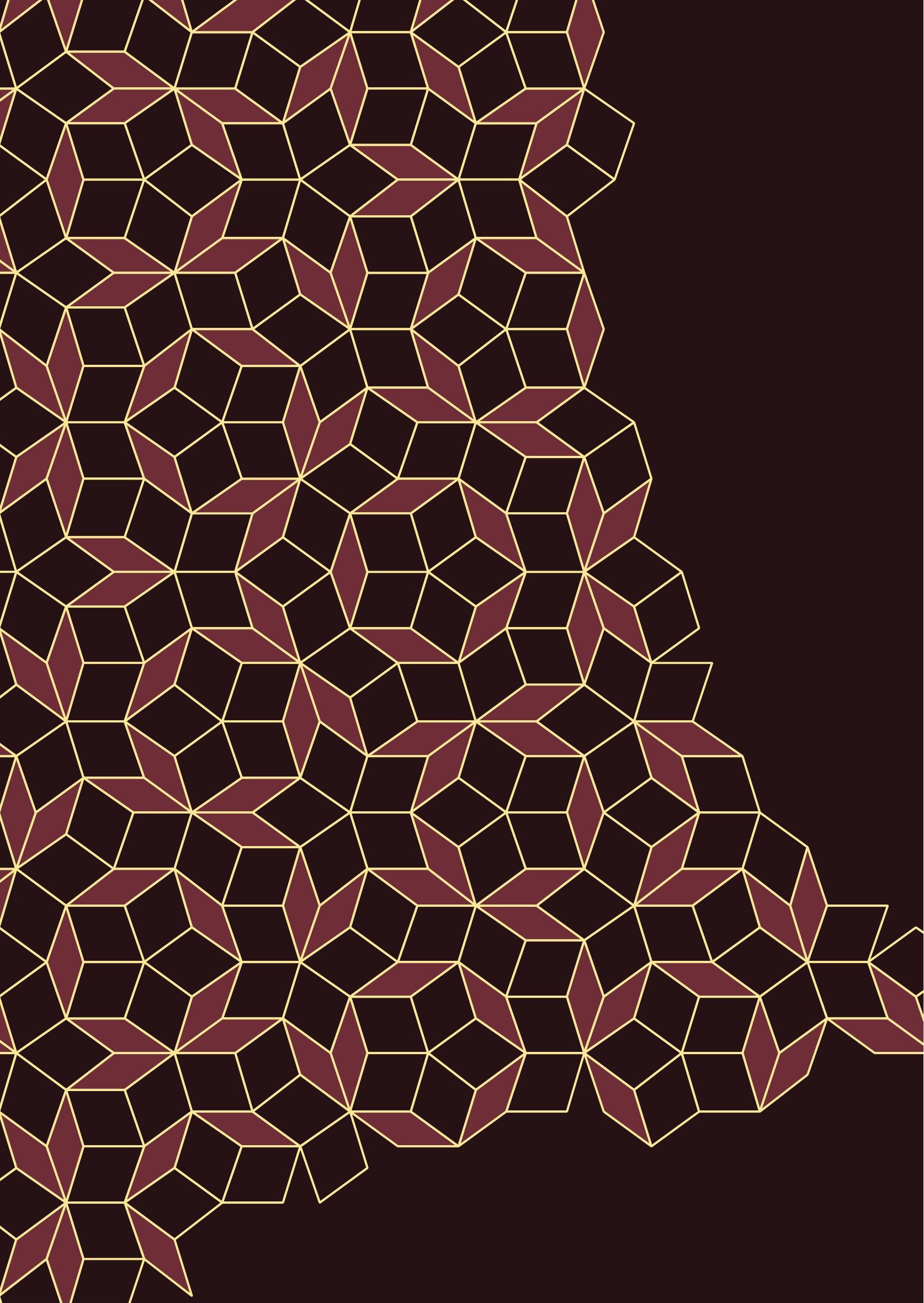

